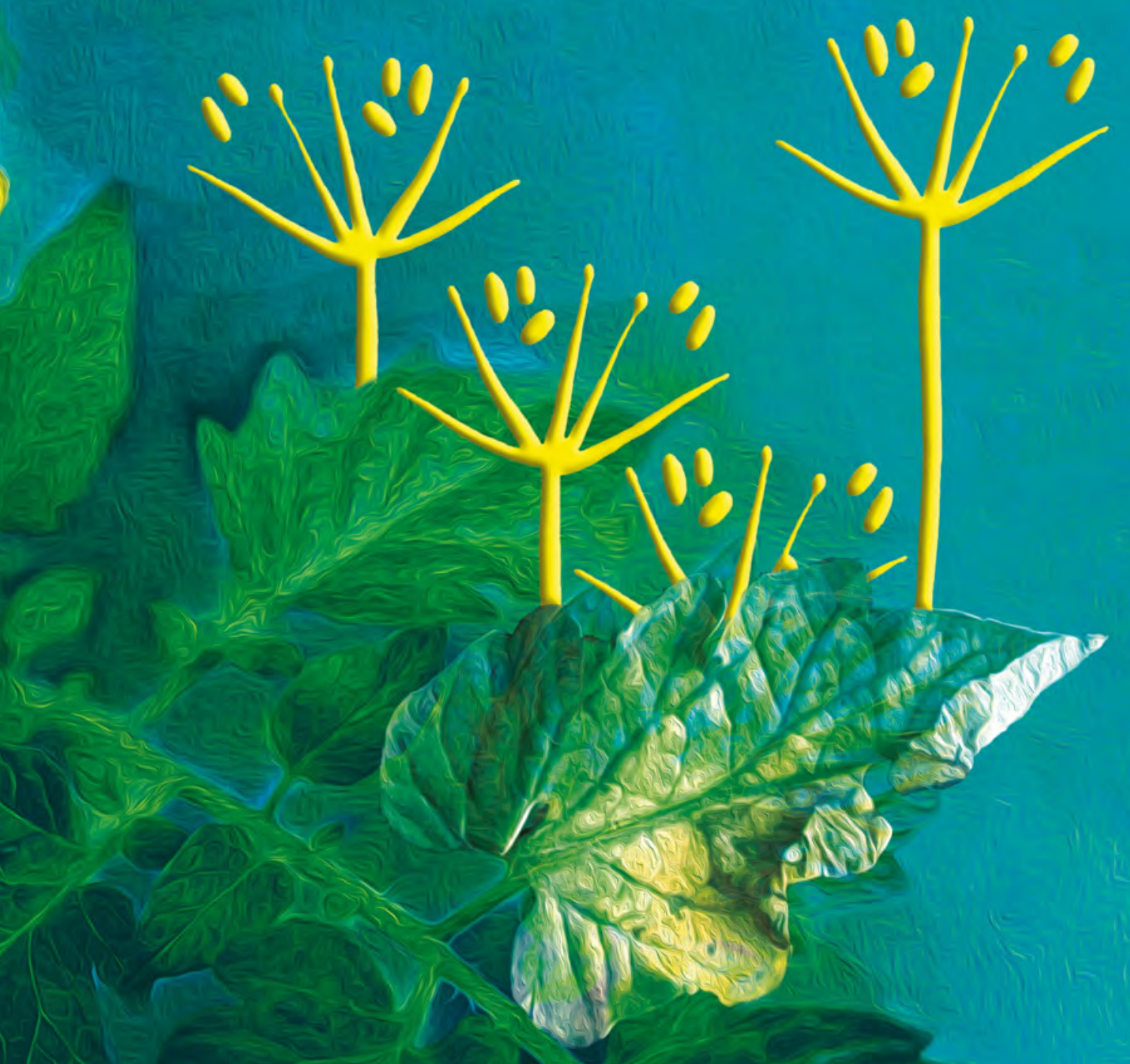

Virulence contribution and recognition of homologs of the Verticillium dahliae effector Ave 1

Jordi C. Boshoven 


\section{Propositions}

1. Verticillium dahliae effector $A v e 1 L$ has been under selection pressure of an immune receptor.

(this thesis)

2. Residues at both the $\mathrm{N}$ - and $\mathrm{C}$-terminus of the Ave 1 effector are required for recognition by tomato immune receptor Ve1.

(this thesis)

3. Organic agriculture alone cannot feed the future world population.

4. We should be more concerned about the impact of pesticides on the environment, than about growing genetically modified crops that can replace pesticides.

5. Competition between scientists for research grants shows parallels with competition between microorganisms in a microbial community since in both cases occupation of a unique niche increases the chances of success.

6. Open access journals should become the forum of science allowing knowledge to become available to anyone interested.

7. Raising a child during a PhD creates a healthy balance between time spent to work and private life.

8. For a Dutchman it is easier to write a PhD thesis than to learn to speak Greek.

Propositions belonging to the thesis, entitled

Virulence contribution and recognition of homologs of the

Verticillium dahliae effector Ave1

Jordi C. Boshoven

Wageningen, 10 November 2017 


\section{Virulence contribution and recognition of homologs of the}

Verticillium dahliae effector Ave 1

Jordi C. Boshoven 


\section{Thesis committee}

\section{Promotors}

Prof. Dr B.P.H.J. Thomma

Professor of Phytopathology

Wageningen University \& Research

Prof. Dr P.J.G.M de Wit

Professor of Phytopathology

Wageningen University \& Research

\section{Other members}

Prof. Dr M.G.M. Aarts, Wageningen University \& Research

Prof. Dr A. Pérez-García, University of Málaga, Spain

Dr Y. Bai, Wageningen University \& Research

Dr M. de Vos, Keygene N.V., Wageningen

This research was conducted under the auspices of the Graduate School Experimental Plant Sciences. 


\title{
Virulence contribution and recognition of homologs of the Verticillium dahliae effector Ave 1
}

\author{
Jordi C. Boshoven
}

Thesis

submitted in fulfilment of the requirements for the degree of doctor at Wageningen University

by the authority of the Rector Magnificus,

Prof. Dr A.P.J. Mol,

in the presence of the

Thesis Committee appointed by the Academic Board to be defended in public

on Friday 10 November 2017

at 11 a.m. in the Aula. 
Jordi C. Boshoven

Virulence contribution and recognition of homologs of the Verticillium dahliae effector Ave1,

184 pages.

PhD thesis, Wageningen University, Wageningen, the Netherlands (2017)

With references, with summary in English

ISBN 978-94-6343-644-1

DOI http://dx.doi.org/10.18174/420169 
Chapter 2 Filamentous Pathogen Effector Functions:

Chapter 3 Homologs of Verticillium dahliae effector Ave1 contribute to virulence in fungal pathogens of diverse plant hosts

Chapter 4 A nine amino acid peptide of Verticillium dahliae effector Ave1 is required but not sufficient for recognition by tomato immune receptor Ve1

Chapter 5 Characterization of an expanded family of Ave1 homologs in the apple and pear scab fungi Venturia inaequalis and $V$. pirina

Chapter 6 An Ave1-like effector displays allelic variation within the Verticillium dahliae population

Chapter 7 Rhamnose synthase activity is required for pathogenicity of Verticillium dahliae

Chapter 8 General discussion: From effector discovery to breeding for durable pathogen resistance in crops

Summary

Acknowledgements

About the author

List of publications 

To my family 

General introduction

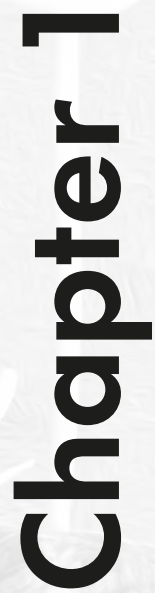


Chapter 1 


\section{Introduction}

Plants provide us with food, feed, fuel, fibre, shelter and drugs and are therefore an important natural resource on which we depend heavily. Since the world population has been, and still is, rapidly growing, the meat and dairy intake has been increasing and also the use of biofuel is rising. Collectively, this still results in an urgent demand for increased crop production. The green revolution that started in the 60s of the $20^{\text {th }}$ century intensified global agricultural practice significantly, often resulting in vast areas of a single crop cultivar known as a monoculture (Jain 2010). Disease outbreaks caused by plant pathogens including fungi, oomycetes, bacteria and viruses can lead to severe yield losses and can even wipe out entire crops, especially when cultivated as monoculture (Oerke 2006; Strange and Scott 2005). To find novel and improved means to combat disease outbreaks, a fundamental understanding of the molecular basis of host-pathogen interactions is essential. Already in the 40 s of the $20^{\text {th }}$ century, Harald Flor showed that plants that carry resistance $(R)$ genes are resistant to pathogens that carry corresponding avirulence (Avr) genes (Flor 1942). Initially, it was thought that $\mathrm{R}$ and Avr proteins directly interact (Keen 1990). However, most protein interaction studies failed to confirm this hypothesis, which led to the proposal of the guard hypothesis (Van der Biezen and Jones 1998). This hypothesis states that an $R$ protein guards a plant protein that, when targeted by a pathogen Avr protein, triggers a resistance response. It subsequently became clear that Avr proteins not only initiate resistance responses, but actually act as pathogen virulence factors that manipulate host proteins in absence of host resistance proteins. Therefore, the term effector is more frequently used for Avr proteins nowadays (Boller and Felix 2009). Effectors are considered to be species-, or even race- or strain-specific (Chisholm et al. 2006). Besides sensing effectors or their activities, plants recognize conserved structural components of microorganisms such as bacterial flagellin, fungal chitin and oomycete $\beta$-glucans, known as microbeassociated molecular patterns (MAMPs) resulting in MAMP-triggered immunity (MTI) (Dodds and Rathjen 2010). It is generally thought that pathogens evolved effectors to overcome this MTI response. In turn, however, plants evolved receptors to recognize these effectors, resulting in effector-triggered immunity (ETI). These receptors are the earlier discussed $R$ proteins. To avoid recognition by the plant, pathogens have to lose or mutate the recognized effector, or evolve new effectors that can again suppress the immune response. These processes continuously occur in the ongoing arms-race that takes place between plants and pathogens (Chisholm et al. 2006; Jones and Dangl 2006). 
About a decade after its introduction, several misconceptions are ubiquitously professed when describing the plant immune system, such as strict separation of MTI versus ETI (Cook et al. 2015; Thomma et al. 2011). Therefore, it was recently proposed that an accurate description of inducible plant immunity should explicitly separate host perception of a given ligand from its physiological or biochemical function. This is captured in the Invasion Model in which host receptors, termed invasion pattern receptors (IPRs), detect ligands that accurately betray pathogen ingress, termed invasion patterns (IPs)(Cook et al. 2015). These IPs can either be an externally encoded or modified-self ligands that indicates invasion. Although any molecule may serve as an IP that will be detected by an IPR, the probability of any given ligand-receptor complex to develop an important role in host immunity increases with increasing ligand molecular constraint to retain function, conservation across organisms, importance in facilitating symbiosis, and accessibility (Cook et al. 2015).

\section{The study system}

The genus Verticillium includes soil-borne fungal vascular pathogens that are causal agents of Verticillium wilts in a broad range of host plants, including many economically important crops (Agrios 2005), leading to billions of euros of damage annually (Pegg and Brady 2002). We use the species $V$. dahliae as a model system since it has a broad host range and, consequently, can infect model plants such as Arabidopsis, tomato and tobacco. The lifecycle of $\mathrm{V}$. dahliae starts with the germination of resting structures, microsclerotia, in the soil after sensing of root exudates. The hyphae that grow from the germinating microsclerotia will progress towards the root and penetrate the root tips (Fitzell et al. 1980; Mol 1995). V. dahliae grows through the root cortex into the xylem vessels where it produces conidia that will further spread through the plant (Bishop and Cooper 1983; Fitzell et al. 1980; Garber and Houston 1966). Growth of the fungus in the xylem vessels blocks water transport, which will ultimately lead to wilting and dying of the tissue. Microsclerotia will form on dying tissue, and will eventually end up in the soil after the decomposition of plant material. V. dahliae is difficult to control since it has a broad host range and microsclerotia can survive up to 20 years in the soil, which makes crop rotation ineffective. Most fungicides are ineffective as well because they do not reach the fungus which grows protected inside the plant. Although soil fumigation is effective against $V$. dahliae most countries have banned this practice due to its detrimental effects on the environment (Fradin and Thomma 2006).

An effective way to control $V$. dahliae is genetic resistance conferred by tomato immune receptor Ve1 of which homologs are found amongst others in particular tobacco genotypes, mint, hop and cotton (Majer et al. 2014; Vining and Davis 2009; Zhang 
et al. 2011; Zhang et al. 2013). Nevertheless, functionality of the Ve1 homologs was only reported in Nicotiana glutinosa and cotton (Zhang et al. 2011; Zhang et al. 2013). Plants carrying Ve 1 are resistant to race 1 strains of $V$. dahliae, but not to race 2 strains (Fradin et al. 2009; Kawchuk et al. 2001). Recently, the effector that is recognized by Ve1 was identified in race 1 strains and named Ave1, for mediating avirulence on Ve1 plants (de Jonge et al. 2012). Besides that Ave1 deletion strains are no longer recognized by Ve1, they show reduced aggressiveness on tomato plants lacking Ve1, indicating a role in virulence (de Jonge et al. 2012). In addition, Ave1 deletion was shown to compromise aggressiveness on Arabidopsis as well (de Jonge, van Esse et al. 2012). Remarkably, homologs of Ave1 are mainly found in plants and only in a few microorganisms that all happen to be plant pathogens, including the fungi Fusarium oxysporum f. sp. lycopersici, Cercospora beticola, Colletotrichum higginsianum and the bacterium Xanthomonas axonopodis (de Jonge et al. 2012). The Ave1 homologs from plant pathogens were coexpressed with Ve1 in Nicotiana tabacum to determine whether they can be recognized as well. This experiment showed that homologs from F. oxysporum and C. beticola are recognized while the homologs from C. higginsianum and $X$. axonopodis are not, or only poorly, recognized (de Jonge et al. 2012). Since F. oxysporum f. sp. lycopersici is a tomato pathogen the fungus was inoculated on transgenic tomato carrying Ve1. These Ve1 tomato plants showed reduced disease symptoms when compared with plants that lack Ve1, suggesting that recognition of FoAve1 activates host defense (de Jonge et al. 2012).

The Ave1 homologs that were found in plants are annotated as plant natriuretic peptides or expansin-like proteins that are described to be involved in ion homeostasis, and therefore in many downstream processes including growth, net water uptake, photosynthesis, stomatal opening and gas exchange (Gehring and Irving 2003; Ludidi et al. 2004; Maryani et al. 2001; Pharmawati et al. 2001). Phylogenetic analysis showed that Ave1 homologs found in microbes do not follow phylogeny of the species in which they occur, but rather occur among plant homologs, which is suggestive of horizontal gene transfer. Therefore, our working hypothesis is that Ave1 homologs found in microbes are acquired from plants through horizontal gene transfer (de Jonge et al. 2012; Gottig et al. 2008). Interestingly, the Ave1 homolog that is found in $X$. axonopodis known as XacPNP is a virulence factor that affects homeostasis and photosynthesis in citrus plants (Garavaglia et al. 2010; Gottig et al. 2008; Nembaware et al. 2004). Since Ave1 homologs are found in multiple plant pathogens and are likely important for virulence, further studies are needed to gain more understanding of the function of these homologs. 


\section{Main objective and research questions}

The main objective of this $\mathrm{PhD}$ research is to study the Ave1 homologs derived from Verticillium species and other plant pathogens. To this end we addressed the following research questions:

- How are the Ave1 homologs distributed?

- What is the role of Ave1 homologs in virulence in the pathogens that express them?

- What is the function of the Ave1 homologs?

- Do all Ave1 homologs display the same functionality?

- What determines recognition of Ave1 homologs by Ve1?

- How did the Ave1 homologs evolve?

\section{Thesis outline}

In Chapter 2, a complete overview of the biological function of fungal effectors is provided. Moreover, the most recent views on various manners how fungal effectors contribute to disease establishment is provided. Furthermore we discuss potential roles of fungal effectors outside host-pathogen interactions.

In Chapter 3, we studied the Ave1 homologs of three fungal pathogens: F. oxysporum f. sp. lycopersici, C. beticola and C. higginsianum that infect tomato, sugarbeet and Arabidopsis, respectively. We determine whether the Ave1 homologs are expressed by the respective fungi during host colonization and whether these genes play a role in virulence.

In Chapter 4, the recognition of $V$. dahliae Ave1 by the tomato Ve1 immune receptor is studied. Based on comparisons of Ave1 homologs from various species and functional assays with truncated proteins and domain swaps, attempts are made to determine the region of Ave1 that is recognized by $V e 1$.

In Chapter 5, the expanded Ave1 gene family that is found in the genomes of the apple and pear scab pathogens Venturia ineaqualis and V. pirina is described. RNAseq of $V$. ineaqualis revealed that some of the paralogs are induced in planta. It is studied whether the induced Ave1 homologs are recognized by Ve1 and whether they may perform the same biological function as $V$. dahliae Ave1.

In Chapter 6, we describe the occurrence of an Ave1-like gene that is found in many $V$. dahliae strains. In our analysis, we identified five different alleles of this gene. We determine whether the various Ave1-like gene alleles are expressed during host colonization, and whether Ve1 or its close homolog $V e 2$ is able to recognize these. 
In Chapter 7, a random mutagenesis was performed in V. dahliae to identify novel virulence and pathogenicity factors. One of the virulence factors that was identified is further characterized.

Chapter $\mathbf{8}$ is the general discussion and describes how the knowledge of pathogen effectors can be used as a tool to establish durable broad spectrum resistance. 


\section{References}

Agrios, G. N. 2005. Plant Pathology. 5th ed. San Diego: Academic Press.

Bishop, C., and Cooper, R. M. 1983. An ultrastructural study of root invasion in three vascular wilt diseases. Physiol Plant Pathol 22:15-27.

Boller, T., and Felix, G. 2009. A renaissance of elicitors: perception of microbe-associated molecular patterns and danger signals by pattern-recognition receptors. Annu Rev Plant Biol 60:379-406.

Chisholm, S. T., Coaker, G., Day, B., and Staskawicz, B. J. 2006. Host-microbe interactions: shaping the evolution of the plant immune response. Cell 124:803-814.

Cook, D. E., Mesarich, C. H., and Thomma, B. P. H. J. 2015. Understanding plant immunity as a surveillance system to detect invasion. Annu Rev Phytopathol.

de Jonge, R., van Esse, H. P., Maruthachalam, K., Bolton, M. D., Santhanam, P., Saber, M. K., Zhang, Z., Usami, T., Lievens, B., Subbarao, K. V., and Thomma, B. P. H. J. 2012. Tomato immune receptor Ve1 recognizes effector of multiple fungal pathogens uncovered by genome and RNA sequencing. Proc Natl Acad Sci U S A 109:5110-5115.

Dodds, P. N., and Rathjen, J. P. 2010. Plant immunity: towards an integrated view of plant-pathogen interactions. Nat Rev Genet 11:539-548.

Fitzell, R., Evans, G., and Fahy, P. 1980. Studies on the colonization of plant roots by Verticillium dahliae Klebahn with use of immunofluorescent staining. Aust J Bot 28:357-368.

Flor, H. H. 1942. Inheritance of pathogenicity in Melampsora lini. Phytopathology 32:653-669.

Fradin, E. F., and Thomma, B. P. H. J. 2006. Physiology and molecular aspects of Verticillium wilt diseases caused by V. dahliae and V. albo-atrum. Mol Plant Pathol 7:71-86.

Fradin, E. F., Zhang, Z., Ayala, J. C. J., Castroverde, C. D., Nazar, R. N., Robb, J., Liu, C.-M., and Thomma, B. P. 2009. Genetic dissection of Verticillium wilt resistance mediated by tomato Ve1. Plant Physiology 150:320-332.

Garavaglia, B. S., Thomas, L., Gottig, N., Dunger, G., Garofalo, C. G., Daurelio, L. D., Ndimba, B., Orellano, E. G., Gehring, C., and Ottado, J. 2010. A eukaryotic-acquired gene by a biotrophic phytopathogen allows prolonged survival on the host by counteracting the shut-down of plant photosynthesis. PLoS One 5:e8950.

Garber, R., and Houston, B. 1966. Penetration and development of Verticillium albo-atrum in cotton plant. Phytopathology 56:1121.

Gehring, C., and Irving, H. 2003. Natriuretic peptides - a class of heterologous molecules in plants. Int J Biochem Cell Biol 35:1318-1322.

Gottig, N., Garavaglia, B. S., Daurelio, L. D., Valentine, A., Gehring, C., Orellano, E. G., and Ottado, J. 2008. Xanthomonas axonopodis pv. citri uses a plant natriuretic peptide-like protein to modify host homeostasis. Proc Natl Acad Sci U S A 105:18631-18636.

Jain, H. 2010. Green revolution: history, impact and future. Studium Press LLC, Houston USA.

Jones, J. D., and Dangl, J. L. 2006. The plant immune system. Nature 444:323-329.

Kawchuk, L. M., Hachey, J., Lynch, D. R., Kulcsar, F., Van Rooijen, G., Waterer, D. R., Robertson, A., Kokko, E., Byers, R., and Howard, R. J. 2001. Tomato Ve disease resistance genes encode cell surfacelike receptors. Proceedings of the National Academy of Sciences of the United States of America 98:6511-6515.

Keen, N. 1990. Gene-for-gene complementarity in plant-pathogen interactions. Annu Rev Gen 24:447463.

Ludidi, N., Morse, M., Sayed, M., Wherrett, T., Shabala, S., and Gehring, C. 2004. A recombinant plant natriuretic peptide causes rapid and spatially differentiated $\mathrm{K}+, \mathrm{Na}+$ and $\mathrm{H}+$ flux changes in Arabidopsis thaliana roots. Plant Cell Physiol 45:1093-1098.

Majer, A., Javornik, B., Cerenak, A., and Jakse, J. 2014. Development of novel EST-derived resistance gene markers in hop (Humulus lupulus L.). Mol Breed 33:61-74. 
Maryani, M., Bradley, G., Cahill, D., and Gehring, C. 2001. Natriuretic peptides and immunoreactants modify osmoticum-dependent volume changes in Solanum tuberosum L. mesophyll cell protoplasts. Plant Sci 161:443-452.

Mol, L. 1995. Effect of plant roots on the germination of microsclerotia of Verticillium dahliae. Eur J Plant Pathol 6:679-685.

Nembaware, V., Seoighe, C., Sayed, M., and Gehring, C. 2004. A plant natriuretic peptide-like gene in the bacterial pathogen Xanthomonas axonopodis may induce hyper-hydration in the plant host: a hypothesis of molecular mimicry. BMC Evol Biol 4:10.

Oerke, E. C. 2006. Crop losses to pests. J Agric Sci 144:31-43.

Pegg, G. F., and Brady, B. L. 2002. Verticillium wilts. CABI, Wallingford.

Pharmawati, M., Maryani, M. M., Nikolakopoulos, T., Gehring, C. A., and Irving, H. R. 2001. Cyclic GMP modulates stomatal opening induced by natriuretic peptides and immunoreactive analogues. Plant Physiol Biochem 39:385-394.

Strange, R. N., and Scott, P. R. 2005. Plant disease: a threat to global food security. Annu Rev Phytopathol 43:83-116.

Thomma, B. P. H. J., Nurnberger, T., and Joosten, M. H. A. J. 2011. Of PAMPs and effectors: The blurred PTI-ETI dichotomy. Plant Cell 23:4-15.

Van der Biezen, E. A., and Jones, J. D. 1998. Plant disease-resistance proteins and the gene-for-gene concept. Trends Biochem Sci 23:454-456.

Vining, K., and Davis, T. 2009. Isolation of a Ve homolog, mVe1, and its relationship to Verticillium wilt resistance in Mentha longifolia (L.) Huds. Mol Genet Genomics 282:173-184.

Zhang, Y., Wang, X., Yang, S., Chi, J., Zhang, G., and Ma, Z. 2011. Cloning and characterization of a Verticillium wilt resistance gene from Gossypium barbadense and functional analysis in Arabidopsis thaliana. Plant Cell Rep 30:2085-2096.

Zhang, Z., Fradin, E., de Jonge, R., van Esse, H. P., Smit, P., Liu, C.-M., and Thomma, B. P. H. J. 2013. Optimized agroinfiltration and virus-induced gene silencing to study Ve1-mediated Verticillium resistance in tobacco. Mol. Plant Microbe Interact. 26:182-190. 



\section{Filamentous Pathogen Effector Functions: Of Pathogens, Hosts and Microbiomes}

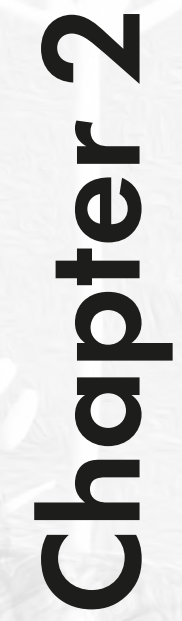

Hanna Rovenich"

Jordi C. Boshoven\#

Bart P.H.J. Thomma

\#These authors contributed equally 
Microorganisms play essential roles in almost every environment on earth. For instance, microbes decompose organic material, or establish symbiotic relationships that range from pathogenic to mutualistic. Symbiotic relationships have been particularly well studied for microbial plant pathogens and have emphasized the role of effectors; secreted molecules that support host colonization. Most effectors characterized thus far play roles in deregulation of host immunity. Arguably, however, pathogens not only deal with immune responses during host colonization, but also encounter other microbes including competitors, (myco)parasites and even potential co-operators. Thus, part of the effector catalog may target microbiome co-inhabitants rather than host physiology. 


\section{Introduction}

During early microbial colonization stages, plant cell surface-localized pattern recognition receptors (PRRs) recognize microbe-associated molecular patterns (MAMPs), such as fungal chitin, to activate immune responses (Dodds and Rathjen 2010; Thomma et al. 2011). In order to establish themselves, adapted pathogens secrete effector molecules that deregulate immune responses and facilitate host colonization. Simultaneously, hosts evolve effector recognition by novel receptors that reinstall immunity (Dodds and Rathjen 2010; Thomma et al. 2011). Consequently, effectors are subject to various selective forces that drive their evolution, leading to diversified effector repertoires between pathogen lineages. Functional characterization of effectors and determination of their contribution to the microbial lifestyle provides insight in relevant processes for host colonization.

\section{Plant pathogen effectors deregulate host immunity in various subcellular compartments}

Many pathogens initially enter the plant apoplast, which contains enzymes that hamper microbial colonization. For example, chitinases target fungal cell walls to release chitin fragments that activate immune receptors, leading to further chitinase accumulation to induce hyphal lysis. In turn, fungal pathogens secrete chitin-binding effectors to protect their cell walls and interfere with immune receptor activation (de Jonge et al. 2010; Kombrink et al. 2011; Marshall et al. 2011; Mentlak et al. 2012). The LysM domain-containing Ecp6 effector of the leaf mold fungus Cladosporium fulvum can outcompete host receptors through chitin binding with unprecedented ultrahigh (pM) affinity by intramolecular LysM domain dimerization (Sanchez-Vallet et al. 2013). Additionally, LysM effectors likely interfere with receptor dimerization that is required to activate immune signaling (Hayafune et al. 2014; Liu et al. 2012; Sanchez-Vallet et al. 2013).

Although effectors that directly target chitinases have not yet been identified, some effectors target other apoplastic hydrolytic enzymes, such as proteases. For example, sequence-unrelated effectors of $C$. fulvum, the oomycete Phytophthora infestans, and the parasitic nematode Globodera rostochiensis inhibit tomato cysteine proteases including Rcr3 (Lozano-Torres et al. 2012; Song et al. 2009; van Esse et al. 2008). The closely related oomycetes $P$. infestans and $P$. mirabilis express an orthologous pair of host protease inhibitor effectors that are subject to positive selection, which was implicated in adaptation to unique protease targets in their respective host plants (Dong et al. 2014). Besides protease inhibitors, P. infestans secretes the Avrblb2 effector that interferes with protease secretion (Bozkurt et al. 
2011). The smut fungus Ustilago maydis inhibits apoplastic proteases via multiple effectors. While Pit2 directly inhibits cysteine proteases (Mueller et al. 2013), Pep1 induces the maize cystatin CC9 that inhibits apoplastic proteases in turn (van der Linde et al. 2012). Pep1 furthermore inhibits the maize peroxidase POX12 to perturb reactive oxygen species balances (Hemetsberger et al. 2012). Thus, the plant apoplast is a dynamic battlefield for plant pathogens.

In addition to apoplastic effectors, many pathogens deliver effectors that act inside host cells, although mechanisms that govern their uptake remain controversial (Petre and Kamoun 2014). The rice blast fungus Magnaporthe oryzae was shown to secrete various effectors that enter rice cells, and even move to non-infected neighboring cells, presumably to prepare these for infection (Khang et al. 2010). The AvrPiz-t effector targets proteasome activity through interaction with the RING E3 ubiquitin ligase APIP6, leading to their mutual degradation and suppression of PRR-mediated immunity (Park et al. 2012). Effector diffusion from infected cells into neighboring cells was similarly observed for the U. maydis chorismate mutase $\mathrm{Cmu} 1$ that targets the shikimate pathway to channel chorismate into the phenylpropanoid pathway, thus adversely affecting salicylic acid (SA) biosynthesis (Djamei et al. 2011). U. maydis furthermore secretes the Tin2 effector to stabilize the maize ZmTTK1 kinase that controls anthocyanin biosynthesis, possibly to suppress tissue lignification (Tanaka et al. 2014). Also the oomycete Hyaloperonospora arabidopsidis targets SA signaling by secreting a nuclear-localized effector that interacts with the mediator complex that controls interactions between transcriptional regulators and RNA polymerase (Caillaud et al. 2013). Host transcription is furthermore perturbed by effectors that inhibit transcription factor translocation to the nucleus (McLellan et al. 2013). Additionally, nuclear-localized effectors may affect host immunity posttranscriptionally by suppressing the biogenesis of small RNAs in the host (Qiao et al. 2013). Interestingly, Botrytis cinerea was recently suggested to deliver even small RNAs into host cells to affect immune responses (Weiberg et al. 2013).

Finally, several effectors target host cell death mechanisms, such as $P$. infestans Avr3a and PexRD2. While Avr3a suppresses INF1-triggered cell death by stabilizing the U-box E3 ligase CMPG1 during biotrophic growth, PexRD2 targets the kinase domain of the cell death regulator MAPKKKE (Bos et al. 2010; King et al. 2014). During later stages of infection, however, P. infestans relies on induction of host cell death as it switches to a necrotrophic lifestyle. Necrotrophic pathogens evolved effectors that actually induce cell death. An elegant example is provided by the Cochliobolus victoriae effector victorin that binds to thioredoxins including TRXh5, which is required for redox control of the transcriptional immune regulator NPR1. TRXh5 binding activates the NB-LRR-type immune receptor LOV1, facilitating necrotrophic exploitation of host cell death by C. victoriae (Lorang et al. 2012). 
In conclusion, although information for the vast majority of pathogen effectors, particularly of filamentous pathogens, is still lacking, effector molecules are highly versatile. Clearly, recently uncovered functions revealed that virulence effectors, despite the finding that they converge onto pivotal elements of the plant immune system (Mukhtar et al. 2011), can deregulate any step of immunity in any cellular compartment (Figure 1, Supplemental table 1).

\section{Endophytes and mutualists use effectors to suppress host immunity too}

Like pathogens, commensalistic endophytes and mutualists develop intimate host plant associations. During initiation of such symbioses, PRRs continue to perceive MAMPs. Consequently, similar to pathogens, endophytes and mutualists are recipients of immune responses. However, the precise role and fate of host immunity in the establishment of symbiosis has remained enigmatic.

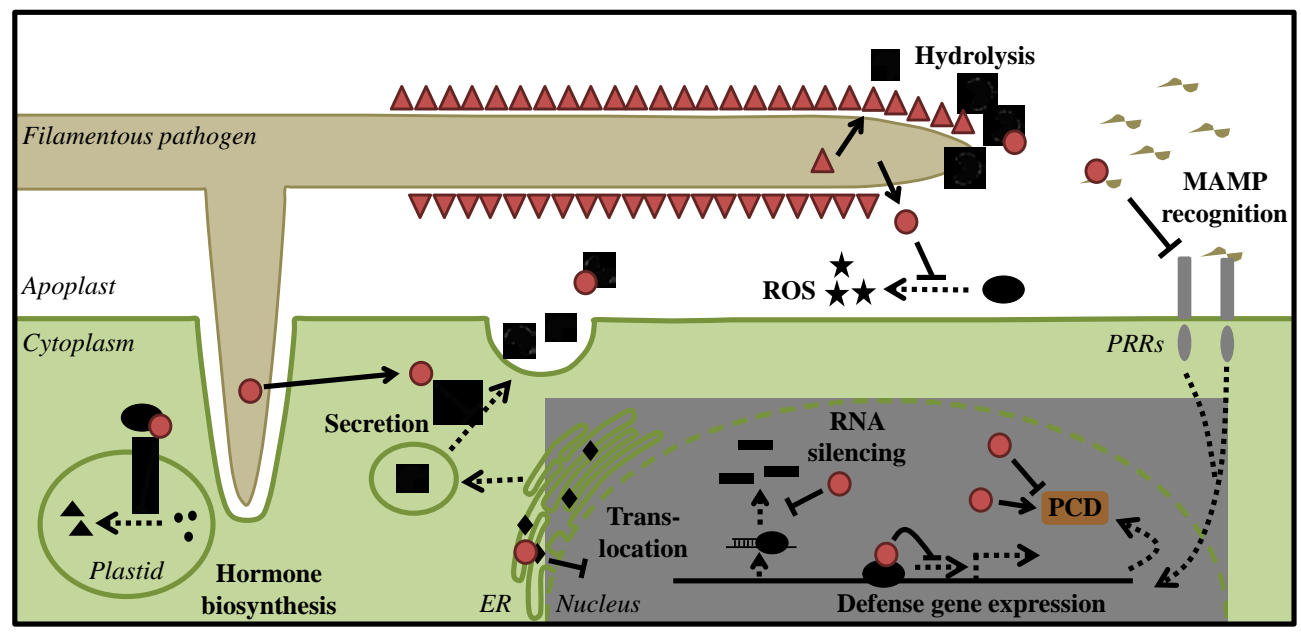

FIGURE 1 | Filamentous pathogen effectors deregulate host immunity in various host subcellular compartments. Pathogens secrete effectors (red symbols) to deregulate plant immunity (see text for details). Whereas one group of effectors (red circles) interacts with host targets that act in immunity (black shapes), another group of effectors (red triangles) acts in self-defense to protect the pathogen from host-derived antimicrobials.

The root endophyte Piriformospora indica has a wide host range and induces enhanced growth and stress resistance in colonized hosts. Rather than evading host detection, the fungus actively suppresses immunity (Jacobs et al. 2011). During early biotrophic growth at the onset of symbiosis, about $10 \%$ of the transcriptome encodes putative effector proteins (Zuccaro et al. 2011). At later growth stages 
the fungus requires host cell death for further colonization, thus resembling hemibiotrophic pathogens such as Mycosphaerella graminicola and M. oryzae. Like C. fulvum, these latter species utilize LysM effectors to suppress immune responses (de Jonge et al. 2010; Marshall et al. 2011; Mentlak et al. 2012). P. indica carries an expanded LysM domain-containing effector repertoire that may similarly act in immune suppression (Zuccaro et al. 2011).

Effector-like proteins are also encoded by genomes of other mutualists (Martin et al. 2010; Plett et al. 2011; Plett and Martin 2011). The ectomycorrhiza Laccaria bicolor genome encodes hundreds of small secreted proteins, several of which are only expressed in symbiotic tissues. Of these, MiSSP7 was shown to translocate to the nucleus of poplar host cells to stabilize the JAZ6 protein and repress jasmonate signaling (Plett et al. 2014; Plett et al. 2011). Likewise, the ectomycorrhiza Tuber melanosporum expresses 125 cysteine-rich small secreted proteins, including a LysM effector, which are highly upregulated during symbiosis (Martin et al. 2010).

It was recently shown that arbuscular endomycorrhizal fungi produce lipochitooligosaccharide mycorrhizal (Myc) factors that stimulate root growth and branching to initiate symbiosis (Maillet et al. 2011). Similar to endophytes and ectomycorrhiza, arbuscular endomycorrhiza secrete effector-like proteins during symbiotic interactions (Kloppholz et al. 2011; Lin et al. 2014; Tisserant et al. 2013). The genome of Rhizophagus irregularis encodes a family of CRN-like proteins that are abundantly found in plant pathogenic Phytophthora spp. (Lin et al. 2014). R. irregularis was furthermore found to encode an effector that interacts with the pathogenesis-related ethylene-responsive transcription factor 19 (ERF19) in the host nucleus to promote mycorrhization, potentially by counteracting MAMP-induced host defense responses that are regulated by ERF19 (Kloppholz et al. 2011).

Collectively, these findings suggest that symbiotic associations that include endophytism, mutualism and parasitism form a continuum in which effectors play essential roles (Supplemental table 1).

\section{Effectors act in self-defense and competition}

The ability to establish symbiosis evolved multiple times in microbes, presumably from saprotrophism, and many plant pathogens still display saprotrophic life stages. Saprotrophs generally reside within the soil where they feed on decaying organic matter in the presence of a rich microbiota. In this environment, microbial competition as well as co-operation occurs (Figure 2). Threats are posed by (myco) parasites and competitors that produce antibiotics with specific or broad-spectrum activities. Consequently, microbes require molecules for self-defense and interaction with other microbiome partners. 


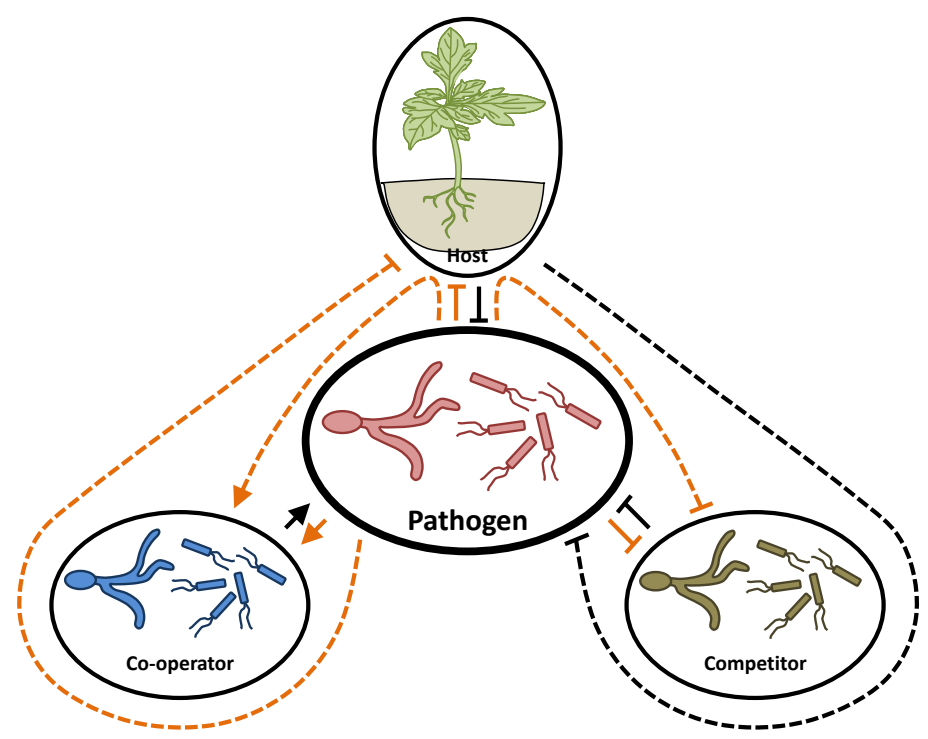

FIGURE 2 | How pathogens influence the local biota by exploiting effector activities. The interaction between microbial pathogens and plant hosts occurs in environments that contain additional microbiome partners that can negatively (competition) or positively (co-operation) impact the pathogen as well as the host. Consequently, the pathogen and host may target each other directly (solid lines) as well as indirectly (dotted lines). Likely, pathogens exploit effector activities (orange lines) to not only directly modulate their hosts, but also to influence the local microbiota that can impact the outcome of the interaction with their hosts.

Similar to infected plants, many mycoparasites secrete hydrolytic enzymes including proteases, chitinases and glucanases to target fungal cell walls. Presumably, chitinbinding effectors that protect hyphal cell walls against plant-derived chitinases similarly protect against mycoparasite-derived chitinases, which may explain abundant LysM effector catalogs of non-pathogenic fungi (Kombrink and Thomma 2013; Kubicek et al. 2011). As LysM domains occur in peptidoglycan-binding proteins of various origins, LysM effector homologs that bind non-chitin substrates likely occur. Indeed, a plant pathogen LysM effector that binds bacterial cell walls was characterized (Kombrink and Thomma, unpublished data), potentially implicating this effector in bacterial competition or protection against bacterial mycoparasites. Genome analyses furthermore revealed that saprotrophic species encode abundant catalogs of small secreted proteins that resemble pathogen effector catalogs (Druzhinina et al. 2012; Kasuga et al. 2009; Kombrink and Thomma 2013; Suh et al. 2012). Although these potential effectors are poorly studied, one such effector, $\mathrm{CipC}$, was implicated in competition with bacteria in Aspergillus spp. (Melin et al. 2002; Suh et al. 2012). The genome of the ubiquitous saprophyte and opportunistic mammalian pathogen A. fumigatus encodes several effector proteins (Wartenberg 
et al. 2011). However, since the vast majority of fungi that cause disease in animals are soil saprophytes that opportunistically infect their hosts, to which they are not highly adapted, it has been speculated that infection does not rely on the activity of effectors (Lowe and Howlett 2012). Rather, their effectors are thought to be required for saprophytic survival (Lowe and Howlett 2012). Nevertheless, effectors that evolved to enable saprophytic survival may be co-opted for opportunistic infection as well.

Likely, competition between plant-associated microbes also occurs within hosts, although perhaps to a lesser extent than in soil due to reduced species diversity. Indeed, the second most abundantly in planta-expressed gene of the fungal endophyte Epichloë festucae encodes a secreted antifungal protein (Ambrose and Belanger 2012). Thus, effector homologs may play crucial roles in microbial competition in a broad spectrum of environments.

\section{Do pathogens shape local microbiomes?}

For various types of multicellular organisms it is increasingly recognized that their microbiome, i.e. the community of microbes that thrives in, on, or immediately near the organism, greatly influences its performance (Ezenwa et al. 2012). For plants, it has been particularly well documented that the rhizosphere microbiota affects plant growth and stress tolerance. In addition, the importance of the phyllosphere microbiota is increasingly recognized (Vorholt 2012). These microbiota comprise members that provide direct as well as indirect pathogen protection through antibiosis and induced immunity, respectively. Whereas soil types have a major impact on root inhabiting bacterial community compositions on Arabidopsis, host genotypes were reported to only have a minor impact (Bulgarelli et al. 2012; Lundberg et al. 2012). In contrast, different Arabidopsis accessions were found to harbor different phyllosphere communities and several host genetic mutations were found to perturb the microbiota composition, demonstrating that host genetic factors shape the associated microbiota (Bodenhausen et al. 2014). It is less clear, however, whether plants evolved to actively recruit phyllosphere communities. Potentially, plants recruit founder species that further shape local microbiomes through inter-microbe interactions (Vorholt 2012). Such interactions may require effectors. Considering that plant factors control the composition of the microbiota, microbiome members may utilize effectors to modulate hosts and control competitors indirectly. Additionally, manipulation of host metabolism could even establish microbial cooperation (Figure 2). Although not immediately addressing inter-microbial interactions, an insect-transmitted phytoplasma was recently shown to utilize an effector to alter floral development of host plants, converting them 
into vegetative tissues that attract leafhopper vectors (MacLean et al. 2014). This represents a striking example of the exploitation of effector activity to influence compositions of the local biome. Similarly, the rust fungus Puccinia monoica induces floral mimicry in the host Boechera stricta to enhance its reproduction and spore dispersal by insects (Cano et al. 2013).

Considering the importance of the microbiome for the ability of plants to withstand pathogen infection, it is conceivable that pathogens evolved to affect host microbiomes, possibly through effector activities (Figure 2).

\section{Different mechanisms drive evolution of effector repertoires}

Mechanisms underlying genome plasticity and evolution have been intensely studied, especially for plant pathogens. As genomes are structured and not just a random sequence of genes, effector genes are often found in dynamic genomic compartments, such as gene-sparse regions, subtelomeric regions or conditionally dispensable (pathogenicity) chromosomes (Raffaele and Kamoun 2012). For example, effector localization in gene-sparse regions was recorded for the endophyte $P$. indica (Zuccaro et al. 2011), while in the saprophyte N. crassa genes encoding small secreted proteins are found in subtelomeric regions (Kasuga et al. 2009). Genetic plasticity in such compartments is governed by diverse mechanisms including recombination and activity of transposable elements. A direct implication of genomic rearrangement in the evolution of fungal aggressiveness was shown for the vascular wilt fungus Verticillium dahliae, leading to the emergence of lineagespecific regions that are enriched for virulence effectors (de Jonge et al. 2013). High genetic variability in effector genes enables rapid evolutionary processes. The importance of dynamic genome compartments for accelerated gene evolution was underlined in the specialization of $P$. infestans after the host jump that separated this species from related species. Uneven evolutionary rates across the genome occur, with in planta-induced genes residing in fast-evolving compartments (Raffaele et al. 2010). In turn, effector specialization can lead to diversification and speciation in pathogen lineages (Dong et al. 2014). In this manner, effectors can determine microbial niches. Moreover, composition of effector catalogs can dictate microbial lifestyles. For example, the leaf epiphyte and antagonist of powdery mildews Pseudozyme flucculosa lost its ability to parasitize plants like its smut fungi relatives due to loss of virulence effectors (Lefebvre et al. 2013). However, the biocontrol agent has acquired other effectors that are not found in the smut relatives that may have shaped its current lifestyle (Lefebvre et al. 2013). These findings suggest that effector catalogs evolve via different mechanisms and that their composition influences a microbe's lifestyle in a given environment. 


\section{Experimental way forward}

The interaction between pathogenic (filamentous) microbes and the organisms they encounter in their niches, either while colonizing the host or during free-living stages in the environment, is poorly understood. An extensive characterization of the complex microbial communities in such niches may lead to a better understanding of the interactions that take place beyond the direct interaction between pathogen and host. Detailed transcriptome analyses may lead to the identification of particular triggers of effector gene expression derived from microbial co-inhabitants, and may hint towards functions in inter-microbial interactions (Mathioni et al. 2013; Mela et al. 2011) that can subsequently be tested for in targeted analysis to reveal components that either promote or inhibit other microbes (Kombrink and Thomma 2013).

\section{Conclusions}

Although a paradigm in plant pathology dictates that existence of disease requires the interaction of a virulent pathogen with a susceptible host in a favorable environment, plant-microbe interactions are mostly studied as one-onone relationships. However, in addition to host immune responses, pathogenic microbes continuously encounter other microbes that include competitors and mycoparasites that need to be dealt with simultaneously. Importantly, findings for pathogenic microbes can be extrapolated to other types of symbioses as well. After all, irrespective of the type of symbiosis, the interest of the microbial partner is merely to exploit the host for nutrition and shelter. This may also explain the thin line that is regularly observed between the different types of symbioses (Delaye et al. 2013; Malcolm et al. 2013; Plett and Martin 2011; Zuccaro et al. 2011). In all types of symbioses, the microbial partner needs to suppress host immune responses and ward off microbial antagonists. Using effectors as probes, further critical processes in host colonization will be uncovered, leading to enhanced understanding of the biology of microbes that aim to establish symbioses.

\section{Acknowledgements}

The authors acknowledge support by the Research Council for Earth and Life sciences (ALW) of the Netherlands Organization for Scientific Research (NWO), thank Melvin Bolton, Andrea Sánchez-Vallet, and Ronnie de Jonge for critically reading the manuscript. 


\section{References}

Ambrose, K. V., and Belanger, F. C. 2012. SOLiD-SAGE of endophyte-infected red fescue reveals numerous effects on host transcriptome and an abundance of highly expressed fungal secreted proteins. PLoS One 7:e53214.

Bodenhausen, N., Bortfeld-Miller, M., Ackermann, M., and Vorholt, J. A. 2014. A synthetic community approach reveals plant genotypes affecting the phyllosphere microbiota. PLoS Genet 10:e1004283.

Bos, J. I., Armstrong, M. R., Gilroy, E. M., Boevink, P. C., Hein, I., Taylor, R. M., Zhendong, T., Engelhardt, S., Vetukuri, R. R., Harrower, B., Dixelius, C., Bryan, G., Sadanandom, A., Whisson, S. C., Kamoun, S., and Birch, P. R. 2010. Phytophthora infestans effector AVR3a is essential for virulence and manipulates plant immunity by stabilizing host E3 ligase CMPG1. Proc Natl Acad Sci U S A 107:9909-9914.

Bozkurt, T. O., Schornack, S., Win, J., Shindo, T., llyas, M., Oliva, R., Cano, L. M., Jones, A. M., Huitema, E., van der Hoorn, R. A., and Kamoun, S. 2011. Phytophthora infestans effector AVRblb2 prevents secretion of a plant immune protease at the haustorial interface. Proc Natl Acad Sci U S A 108:20832-20837.

Bulgarelli, D., Rott, M., Schlaeppi, K., Ver Loren van Themaat, E., Ahmadinejad, N., Assenza, F., Rauf, P., Huettel, B., Reinhardt, R., Schmelzer, E., Peplies, J., Gloeckner, F. O., Amann, R., Eickhorst, T., and Schulze-Lefert, P. 2012. Revealing structure and assembly cues for Arabidopsis root-inhabiting bacterial microbiota. Nature 488:91-95.

Caillaud, M. C., Asai, S., Rallapalli, G., Piquerez, S., Fabro, G., and Jones, J. D. 2013. A downy mildew effector attenuates salicylic acid-triggered immunity in Arabidopsis by interacting with the host mediator complex. PLoS Biol 11:e1001732.

Cano, L. M., Raffaele, S., Haugen, R. H., Saunders, D. G., Leonelli, L., MacLean, D., Hogenhout, S. A., and Kamoun, S. 2013. Major transcriptome reprogramming underlies floral mimicry induced by the rust fungus Puccinia monoica in Boechera stricta. PLoS One 8:e75293.

de Jonge, R., Bolton, M. D., Kombrink, A., van den Berg, G. C., Yadeta, K. A., and Thomma, B. P. H. J. 2013. Extensive chromosomal reshuffling drives evolution of virulence in an asexual pathogen. Genome Res 23:1271-1282.

de Jonge, R., van Esse, H. P., Kombrink, A., Shinya, T., Desaki, Y., Bours, R., van der Krol, S., Shibuya, N., Joosten, M. H., and Thomma, B. P. H. J. 2010. Conserved fungal LysM effector Ecp6 prevents chitin-triggered immunity in plants. Science 329:953-955.

Delaye, L., García-Guzmán, G., and Heil, M. 2013. Endophytes versus biotrophic and necrotrophic pathogens-are fungal lifestyles evolutionarily stable traits? Fungal Divers 60:125-135.

Djamei, A., Schipper, K., Rabe, F., Ghosh, A., Vincon, V., Kahnt, J., Osorio, S., Tohge, T., Fernie, A. R., Feussner, I., Feussner, K., Meinicke, P., Stierhof, Y. D., Schwarz, H., Macek, B., Mann, M., and Kahmann, R. 2011. Metabolic priming by a secreted fungal effector. Nature 478:395-398.

Dodds, P. N., and Rathjen, J. P. 2010. Plant immunity: towards an integrated view of plant-pathogen interactions. Nat Rev Genet 11:539-548.

Dong, S., Stam, R., Cano, L. M., Song, J., Sklenar, J., Yoshida, K., Bozkurt, T. O., Oliva, R., Liu, Z., Tian, M., Win, J., Banfield, M. J., Jones, A. M., van der Hoorn, R. A., and Kamoun, S. 2014. Effector specialization in a lineage of the Irish potato famine pathogen. Science 343:552-555.

Druzhinina, I. S., Shelest, E., and Kubicek, C. P. 2012. Novel traits of Trichoderma predicted through the analysis of its secretome. FEMS Microbiol Lett 337:1-9.

Ezenwa, V. O., Gerardo, N. M., Inouye, D. W., Medina, M., and Xavier, J. B. 2012. Microbiology. Animal behavior and the microbiome. Science 338:198-199.

Hayafune, M., Berisio, R., Marchetti, R., Silipo, A., Kayama, M., Desaki, Y., Arima, S., Squeglia, F., Ruggiero, A., Tokuyasu, K., Molinaro, A., Kaku, H., and Shibuya, N. 2014. Chitin-induced activation of immune signaling by the rice receptor CEBiP relies on a unique sandwich-type dimerization. Proc Natl Acad Sci U S A 111:E404-413. 
Hemetsberger, C., Herrberger, C., Zechmann, B., Hillmer, M., and Doehlemann, G. 2012. The Ustilago maydis effector Pep1 suppresses plant immunity by inhibition of host peroxidase activity. PLoS Pathog 8:e1002684.

Jacobs, S., Zechmann, B., Molitor, A., Trujillo, M., Petutschnig, E., Lipka, V., Kogel, K. H., and Schafer, P. 2011. Broad-spectrum suppression of innate immunity is required for colonization of Arabidopsis roots by the fungus Piriformospora indica. Plant Physiol 156:726-740.

Kasuga, T., Mannhaupt, G., and Glass, N. L. 2009. Relationship between phylogenetic distribution and genomic features in Neurospora crassa. PLoS One 4:e5286.

Khang, C. H., Berruyer, R., Giraldo, M. C., Kankanala, P., Park, S. Y., Czymmek, K., Kang, S., and Valent, B. 2010. Translocation of Magnaporthe oryzae effectors into rice cells and their subsequent cell-to-cell movement. Plant Cell 22:1388-1403.

King, S. R., McLellan, H., Boevink, P. C., Armstrong, M. R., Bukharova, T., Sukarta, O., Win, J., Kamoun, S., Birch, P. R., and Banfield, M. J. 2014. Phytophthora infestans RXLR effector PexRD2 interacts with host MAPKKK epsilon to suppress plant immune signaling. Plant Cell 26:1345-1359.

Kloppholz, S., Kuhn, H., and Requena, N. 2011. A secreted fungal effector of Glomus intraradices promotes symbiotic biotrophy. Curr Biol 21:1204-1209.

Kombrink, A., and Thomma, B. P. H. J. 2013. LysM effectors: secreted proteins supporting fungal life. PLoS Pathog 9:e1003769.

Kombrink, A., Sanchez-Vallet, A., and Thomma, B. P. H. J. 2011. The role of chitin detection in plantpathogen interactions. Microbes Infect 13:1168-1176.

Kubicek, C. P., Herrera-Estrella, A., Seidl-Seiboth, V., Martinez, D. A., Druzhinina, I. S., Thon, M., Zeilinger, S., Casas-Flores, S., Horwitz, B. A., Mukherjee, P. K., Mukherjee, M., Kredics, L., Alcaraz, L. D., Aerts, A., Antal, Z., Atanasova, L., Cervantes-Badillo, M. G., Challacombe, J., Chertkov, O., McCluskey, K., Coulpier, F., Deshpande, N., von Dohren, H., Ebbole, D. J., Esquivel-Naranjo, E. U., Fekete, E., Flipphi, M., Glaser, F., Gomez-Rodriguez, E. Y., Gruber, S., Han, C., Henrissat, B., Hermosa, R., Hernandez-Onate, M., Karaffa, L., Kosti, I., Le Crom, S., Lindquist, E., Lucas, S., Lubeck, M., Lubeck, P. S., Margeot, A., Metz, B., Misra, M., Nevalainen, H., Omann, M., Packer, N., Perrone, G., Uresti-Rivera, E. E., Salamov, A., Schmoll, M., Seiboth, B., Shapiro, H., Sukno, S., Tamayo-Ramos, J. A., Tisch, D., Wiest, A., Wilkinson, H. H., Zhang, M., Coutinho, P. M., Kenerley, C. M., Monte, E., Baker, S. E., and Grigoriev, I. V. 2011. Comparative genome sequence analysis underscores mycoparasitism as the ancestral life style of Trichoderma. Genome Biol 12:R40.

Lefebvre, F., Joly, D. L., Labbe, C., Teichmann, B., Linning, R., Belzile, F., Bakkeren, G., and Belanger, R. R. 2013. The transition from a phytopathogenic smut ancestor to an anamorphic biocontrol agent deciphered by comparative whole-genome analysis. Plant Cell 25:1946-1959.

Lin, K., Limpens, E., Zhang, Z., Ivanov, S., Saunders, D. G., Mu, D., Pang, E., Cao, H., Cha, H., Lin, T., Zhou, Q., Shang, Y., Li, Y., Sharma, T., van Velzen, R., de Ruijter, N., Aanen, D. K., Win, J., Kamoun, S., Bisseling, T., Geurts, R., and Huang, S. 2014. Single nucleus genome sequencing reveals high similarity among nuclei of an endomycorrhizal fungus. PLoS Genet 10:e1004078.

Liu, T., Liu, Z., Song, C., Hu, Y., Han, Z., She, J., Fan, F., Wang, J., Jin, C., Chang, J., Zhou, J. M., and Chai, J. 2012. Chitin-induced dimerization activates a plant immune receptor. Science 336:1160-1164.

Lorang, J., Kidarsa, T., Bradford, C. S., Gilbert, B., Curtis, M., Tzeng, S. C., Maier, C. S., and Wolpert, T. J. 2012. Tricking the guard: exploiting plant defense for disease susceptibility. Science 338:659-662.

Lowe, R. G., and Howlett, B. J. 2012. Indifferent, affectionate, or deceitful: lifestyles and secretomes of fungi. PLoS Pathog 8:e1002515.

Lozano-Torres, J. L., Wilbers, R. H. P., Gawronski, P., Boshoven, J. C., Finkers-Tomczak, A., Cordewener, J. H. G., America, A. H. P., Overmars, H. A., Van 't Klooster, J. W., Baranowski, L., Sobczak, M., llyas, M., van der Hoorn, R. A. L., Schots, A., de Wit, P. J. G. M., Bakker, J., Goverse, A., and Smant, G. 2012. Dual disease resistance mediated by the immune receptor Cf- 2 in tomato requires a common virulence target of a fungus and a nematode. Proc Natl Acad Sci U S A 109:10119-10124. 
Lundberg, D. S., Lebeis, S. L., Paredes, S. H., Yourstone, S., Gehring, J., Malfatti, S., Tremblay, J., Engelbrektson, A., Kunin, V., del Rio, T. G., Edgar, R. C., Eickhorst, T., Ley, R. E., Hugenholtz, P., Tringe, S. G., and Dangl, J. L. 2012. Defining the core Arabidopsis thaliana root microbiome. Nature 488:86-90.

MacLean, A. M., Orlovskis, Z., Kowitwanich, K., Zdziarska, A. M., Angenent, G. C., Immink, R. G., and Hogenhout, S. A. 2014. Phytoplasma effector SAP54 hijacks plant reproduction by degrading MADS-box proteins and promotes insect colonization in a RAD23-dependent manner. PLoS Biol 12:e1001835.

Maillet, F., Poinsot, V., Andre, O., Puech-Pages, V., Haouy, A., Gueunier, M., Cromer, L., Giraudet, D., Formey, D., Niebel, A., Martinez, E. A., Driguez, H., Becard, G., and Denarie, J. 2011. Fungal lipochitooligosaccharide symbiotic signals in arbuscular mycorrhiza. Nature 469:58-63.

Malcolm, G. M., Kuldau, G. A., Gugino, B. K., and Jimenez-Gasco Mdel, M. 2013. Hidden host plant associations of soilborne fungal pathogens: an ecological perspective. Phytopathology 103:538-544.

Marshall, R., Kombrink, A., Motteram, J., Loza-Reyes, E., Lucas, J., Hammond-Kosack, K. E., Thomma, B. P. H. J., and Rudd, J. J. 2011. Analysis of two in planta expressed LysM effector homologs from the fungus Mycosphaerella graminicola reveals novel functional properties and varying contributions to virulence on wheat. Plant Physiol 156:756-769.

Martin, F., Kohler, A., Murat, C., Balestrini, R., Coutinho, P. M., Jaillon, O., Montanini, B., Morin, E., Noel, B., Percudani, R., Porcel, B., Rubini, A., Amicucci, A., Amselem, J., Anthouard, V., Arcioni, S., Artiguenave, F., Aury, J. M., Ballario, P., Bolchi, A., Brenna, A., Brun, A., Buee, M., Cantarel, B., Chevalier, G., Couloux, A., Da Silva, C., Denoeud, F., Duplessis, S., Ghignone, S., Hilselberger, B., lotti, M., Marcais, B., Mello, A., Miranda, M., Pacioni, G., Quesneville, H., Riccioni, C., Ruotolo, R., Splivallo, R., Stocchi, V., Tisserant, E., Viscomi, A. R., Zambonelli, A., Zampieri, E., Henrissat, B., Lebrun, M. H., Paolocci, F., Bonfante, P., Ottonello, S., and Wincker, P. 2010. Perigord black truffle genome uncovers evolutionary origins and mechanisms of symbiosis. Nature 464:1033-1038.

Mathioni, S. M., Patel, N., Riddick, B., Sweigard, J. A., Czymmek, K. J., Caplan, J. L., Kunjeti, S. G., Kunjeti, S., Raman, V., Hillman, B. I., Kobayashi, D. Y., and Donofrio, N. M. 2013. Transcriptomics of the rice blast fungus Magnaporthe oryzae in response to the bacterial antagonist Lysobacter enzymogenes reveals candidate fungal defense response genes. PLoS One 8:e76487.

McLellan, H., Boevink, P. C., Armstrong, M. R., Pritchard, L., Gomez, S., Morales, J., Whisson, S. C., Beynon, J. L., and Birch, P. R. 2013. An RxLR effector from Phytophthora infestans prevents re-localisation of two plant NAC transcription factors from the endoplasmic reticulum to the nucleus. PLoS Pathog 9:e1003670.

Mela, F., Fritsche, K., de Boer, W., van Veen, J. A., de Graaff, L. H., van den Berg, M., and Leveau, J. H. 2011. Dual transcriptional profiling of a bacterial/fungal confrontation: Collimonas fungivorans versus Aspergillus niger. ISME J 5:1494-1504.

Melin, P., Schnurer, J., and Wagner, E. G. 2002. Proteome analysis of Aspergillus nidulans reveals proteins associated with the response to the antibiotic concanamycin A, produced by Streptomyces species. Mol Genet Genomics 267:695-702.

Mentlak, T. A., Kombrink, A., Shinya, T., Ryder, L. S., Otomo, I., Saitoh, H., Terauchi, R., Nishizawa, Y., Shibuya, N., Thomma, B. P. H. J., and Talbot, N. J. 2012. Effector-mediated suppression of chitintriggered immunity by magnaporthe oryzae is necessary for rice blast disease. Plant Cell 24:322-335.

Mueller, A. N., Ziemann, S., Treitschke, S., Assmann, D., and Doehlemann, G. 2013. Compatibility in the Ustilago maydis-maize interaction requires inhibition of host cysteine proteases by the fungal effector Pit2. PLoS Pathog 9:e1003177.

Mukhtar, M. S., Carvunis, A. R., Dreze, M., Epple, P., Steinbrenner, J., Moore, J., Tasan, M., Galli, M., Hao, T., Nishimura, M. T., Pevzner, S. J., Donovan, S. E., Ghamsari, L., Santhanam, B., Romero, V., Poulin, M. M., Gebreab, F., Gutierrez, B. J., Tam, S., Monachello, D., Boxem, M., Harbort, C. J., McDonald, N., Gai, L., Chen, H., He, Y., European Union Effectoromics, C., Vandenhaute, J., Roth, F. P., Hill, D. E., Ecker, J. R., Vidal, M., Beynon, J., Braun, P., and Dangl, J. L. 2011. Independently evolved virulence effectors converge onto hubs in a plant immune system network. Science 333:596-601. 
Okmen, B., Etalo, D. W., Joosten, M. H., Bouwmeester, H. J., de Vos, R. C., Collemare, J., and de Wit, P. J. G. M. 2013. Detoxification of alpha-tomatine by Cladosporium fulvum is required for full virulence on tomato. New Phytol 198:1203-1214.

Park, C. H., Chen, S., Shirsekar, G., Zhou, B., Khang, C. H., Songkumarn, P., Afzal, A. J., Ning, Y., Wang, R., Bellizzi, M., Valent, B., and Wang, G. L. 2012. The Magnaporthe oryzae effector AvrPiz-t targets the RING E3 ubiquitin ligase APIP6 to suppress pathogen-associated molecular pattern-triggered immunity in rice. Plant Cell 24:4748-4762.

Petre, B., and Kamoun, S. 2014. How do filamentous pathogens deliver effector proteins into plant cells? PLoS Biol 12:e1001801.

Plett, J. M., and Martin, F. 2011. Blurred boundaries: lifestyle lessons from ectomycorrhizal fungal genomes. Trends Genet 27:14-22.

Plett, J. M., Kemppainen, M., Kale, S. D., Kohler, A., Legue, V., Brun, A., Tyler, B. M., Pardo, A. G., and Martin, F. 2011. A secreted effector protein of Laccaria bicolor is required for symbiosis development. Curr Biol 21:1197-1203.

Plett, J. M., Daguerre, Y., Wittulsky, S., Vayssieres, A., Deveau, A., Melton, S. J., Kohler, A., Morrell-Falvey, J. L., Brun, A., Veneault-Fourrey, C., and Martin, F. 2014. Effector MiSSP7 of the mutualistic fungus Laccaria bicolor stabilizes the Populus JAZ6 protein and represses jasmonic acid (JA) responsive genes. Proc Natl Acad Sci U S A 111:8299-8304.

Pretsch, K., Kemen, A., Kemen, E., Geiger, M., Mendgen, K., and Voegele, R. 2013. The rust transferred proteins-a new family of effector proteins exhibiting protease inhibitor function. Mol Plant Pathol 14:96-107.

Qiao, Y., Liu, L., Xiong, Q., Flores, C., Wong, J., Shi, J., Wang, X., Liu, X., Xiang, Q., Jiang, S., Zhang, F., Wang, Y., Judelson, H. S., Chen, X., and Ma, W. 2013. Oomycete pathogens encode RNA silencing suppressors. Nat Genet 45:330-333.

Raffaele, S., and Kamoun, S. 2012. Genome evolution in filamentous plant pathogens: why bigger can be better. Nat Rev Microbiol 10:417-430.

Raffaele, S., Farrer, R. A., Cano, L. M., Studholme, D. J., MacLean, D., Thines, M., Jiang, R. H., Zody, M. C., Kunjeti, S. G., Donofrio, N. M., Meyers, B. C., Nusbaum, C., and Kamoun, S. 2010. Genome evolution following host jumps in the Irish potato famine pathogen lineage. Science 330:1540-1543.

Rose, J. K., Ham, K. S., Darvill, A. G., and Albersheim, P. 2002. Molecular cloning and characterization of glucanase inhibitor proteins: coevolution of a counterdefense mechanism by plant pathogens. Plant Cell 14:1329-1345.

Sanchez-Vallet, A., Saleem-Batcha, R., Kombrink, A., Hansen, G., Valkenburg, D. J., Thomma, B. P. H. J., and Mesters, J. R. 2013. Fungal effector Ecp6 outcompetes host immune receptor for chitin binding through intrachain LysM dimerization. Elife 2:e00790.

Schmidt, S. M., Kuhn, H., Micali, C., Liller, C., Kwaaitaal, M., and Panstruga, R. 2014. Interaction of a Blumeria graminis f. sp. hordei effector candidate with a barley ARF-GAP suggests that host vesicle trafficking is a fungal pathogenicity target. Mol Plant Pathol 15:535-549.

Shabab, M., Shindo, T., Gu, C., Kaschani, F., Pansuriya, T., Chintha, R., Harzen, A., Colby, T., Kamoun, S., and van der Hoorn, R. A. 2008. Fungal effector protein AVR2 targets diversifying defense-related cys proteases of tomato. Plant Cell 20:1169-1183.

Song, J., Win, J., Tian, M., Schornack, S., Kaschani, F., llyas, M., van der Hoorn, R. A., and Kamoun, S. 2009. Apoplastic effectors secreted by two unrelated eukaryotic plant pathogens target the tomato defense protease Rcr3. Proc Natl Acad Sci U S A 106:1654-1659.

Stergiopoulos, I., van den Burg, H. A., Okmen, B., Beenen, H. G., van Liere, S., Kema, G. H., and de Wit, P. J. 2010. Tomato Cf resistance proteins mediate recognition of cognate homologous effectors from fungi pathogenic on dicots and monocots. Proceedings of the National Academy of Sciences of the United States of America 107:7610-7615. 
Suh, M. J., Fedorova, N. D., Cagas, S. E., Hastings, S., Fleischmann, R. D., Peterson, S. N., Perlin, D. S., Nierman, W. C., Pieper, R., and Momany, M. 2012. Development stage-specific proteomic profiling uncovers small, lineage specific proteins most abundant in the Aspergillus Fumigatus conidial proteome. Proteome Sci 10:30.

Tanaka, S., Brefort, T., Neidig, N., Djamei, A., Kahnt, J., Vermerris, W., Koenig, S., Feussner, K., Feussner, I., and Kahmann, R. 2014. A secreted Ustilago maydis effector promotes virulence by targeting anthocyanin biosynthesis in maize. Elife 3:e01355.

Thomma, B. P. H. J., Nurnberger, T., and Joosten, M. H. A. J. 2011. Of PAMPs and effectors: The blurred PTI-ETI dichotomy. Plant Cell 23:4-15.

Tian, M., Benedetti, B., and Kamoun, S. 2005. A Second Kazal-like protease inhibitor from Phytophthora infestans inhibits and interacts with the apoplastic pathogenesis-related protease P69B of tomato. Plant Physiol 138:1785-1793.

Tian, M., Huitema, E., Da Cunha, L., Torto-Alalibo, T., and Kamoun, S. 2004. A Kazal-like extracellular serine protease inhibitor from Phytophthora infestans targets the tomato pathogenesis-related protease P69B. J Biol Chem 279:26370-26377.

Tian, M., Win, J., Song, J., van der Hoorn, R., van der Knaap, E., and Kamoun, S. 2007. A Phytophthora infestans cystatin-like protein targets a novel tomato papain-like apoplastic protease. Plant Physiol 143:364-377.

Tisserant, E., Malbreil, M., Kuo, A., Kohler, A., Symeonidi, A., Balestrini, R., Charron, P., Duensing, N., Frei dit Frey, N., Gianinazzi-Pearson, V., Gilbert, L. B., Handa, Y., Herr, J. R., Hijri, M., Koul, R., Kawaguchi, M., Krajinski, F., Lammers, P. J., Masclaux, F. G., Murat, C., Morin, E., Ndikumana, S., Pagni, M., Petitpierre, D., Requena, N., Rosikiewicz, P., Riley, R., Saito, K., San Clemente, H., Shapiro, H., van Tuinen, D., Becard, G., Bonfante, P., Paszkowski, U., Shachar-Hill, Y. Y., Tuskan, G. A., Young, J. P., Sanders, I. R., Henrissat, B., Rensing, S. A., Grigoriev, I. V., Corradi, N., Roux, C., and Martin, F. 2013. Genome of an arbuscular mycorrhizal fungus provides insight into the oldest plant symbiosis. Proc Natl Acad Sci U S A 110:20117-20122.

van den Burg, H. A., Harrison, S. J., Joosten, M. H., Vervoort, J., and de Wit, P. J. G. M. 2006. Cladosporium fulvum Avr4 protects fungal cell walls against hydrolysis by plant chitinases accumulating during infection. Mol. Plant Microbe Interact. 19:1420-1430.

van der Linde, K., Hemetsberger, C., Kastner, C., Kaschani, F., van der Hoorn, R. A., Kumlehn, J., and Doehlemann, G. 2012. A maize cystatin suppresses host immunity by inhibiting apoplastic cysteine proteases. Plant Cell 24:1285-1300.

van Esse, H. P., Van't Klooster, J. W., Bolton, M. D., Yadeta, K. A., van Baarlen, P., Boeren, S., Vervoort, J., de Wit, P. J. G. M., and Thomma, B. P. H. J. 2008. The Cladosporium fulvum virulence protein Avr2 inhibits host proteases required for basal defense. Plant Cell 20:1948-1963.

Vorholt, J. A. 2012. Microbial life in the phyllosphere. Nat Rev Microbiol 10:828-840.

Wartenberg, D., Lapp, K., Jacobsen, I. D., Dahse, H. M., Kniemeyer, O., Heinekamp, T., and Brakhage, A. A. 2011. Secretome analysis of Aspergillus fumigatus reveals Asp-hemolysin as a major secreted protein. Int J Med Microbiol 301:602-611.

Weiberg, A., Wang, M., Lin, F. M., Zhao, H., Zhang, Z., Kaloshian, I., Huang, H. D., and Jin, H. 2013. Fungal small RNAs suppress plant immunity by hijacking host RNA interference pathways. Science 342:118-123.

Zuccaro, A., Lahrmann, U., Guldener, U., Langen, G., Pfiffi, S., Biedenkopf, D., Wong, P., Samans, B., Grimm, C., Basiewicz, M., Murat, C., Martin, F., and Kogel, K. H. 2011. Endophytic life strategies decoded by genome and transcriptome analyses of the mutualistic root symbiont Piriformospora indica. PLoS Pathog 7:e1002290. 


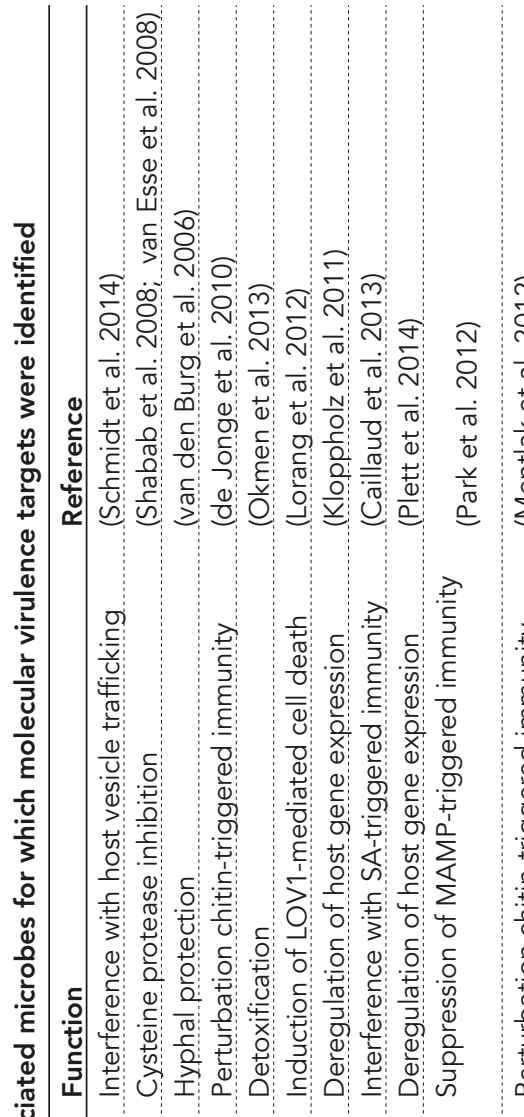

oิ

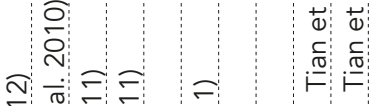

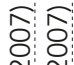

के

m $\bar{c}$

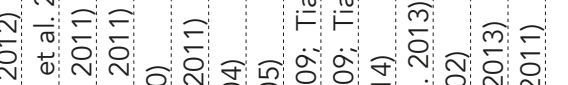

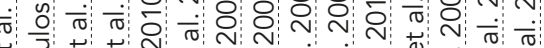

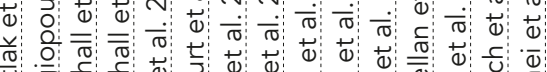

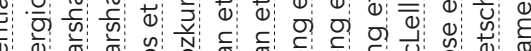

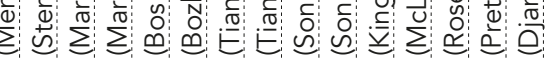

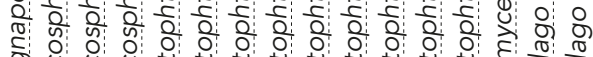

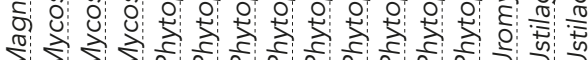

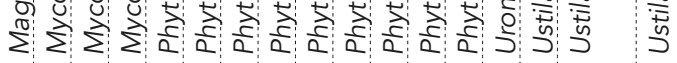




\section{Homologs of Verticillium dahliae effector Ave 1 contribute to virulence of fungal pathogens of diverse plant hosts}

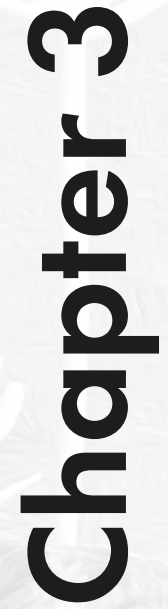

Jordi C. Boshoven

Malaika K. Ebert\#

Yin Song\#

Hanna Rovenich

Eduardo Rojas-Padilla

Melvin D. Bolton

Bart P. H. J. Thomma

\#These authors contributed equally 
Verticillium dahliae is a soil-borne fungal pathogen with a wide host range including many crops. Tomato immune receptor Ve1 confers resistance to $V$. dahliae race 1 strains that express $V d A v e 1$. On plants that do not carry Ve1, Ave1 acts as a virulence factor of $V$. dahliae. Homologs of Ave1 are mostly found in plants and in a handful of fungal plant pathogens including Fusarium oxysporum (FoAve1), Cercospora beticola (CbAve1), and Colletotrichum higginsianum (ChAve1). In the bacterium Xanthomonas axonopodis the Ave1 homolog XacPNP was previously described as a virulence factor. The homologs FoAve1, CbAve1 are recognized by Ve1 although not as efficient as VdAve1. In this study, we analysed the role of the individual Ave1 homologs in virulence. We generated targeted gene deletions in Fusarium oxysporum, Cercospora beticola and Colletotrichum higginsianum and showed that both FoAve 1 and CbAve1 are virulence factors in contrast to ChAve1 that does not contribute to the virulence of the pathogen. We subsequently tested whether Ave1 homologs FoAve1, CbAve1, ChAve 1 and XacPNP act in a similar fashion as VdAve1 by expressing these genes in a $V$. dahliae $V d A v e 1$ deletion strain. We concluded that VdAve1 on the one hand and FoAve1, CbAve1, ChAve1 and XacPNP on the other hand act in different manners. 


\section{Introduction}

Plants are surrounded by microbes including oomycetes, fungi, bacteria and viruses. To prevent colonization by harmful microbes, plants evolved pattern recognition receptors (PRRs) that recognize microbe associated molecular patterns (MAMPs) and activate MAMP-triggered immunity (MTI) (Boller and Felix 2009; Dodds and Rathjen 2010). MAMPs generally are structural components such as bacterial elongation factor Tu (EF-Tu), flagellin, lipopolysaccharides (LPS), fungal chitin and oomycete $\beta$-glucans, which are typically conserved across genera of microbes. Successful pathogens secrete effector proteins that modulate host immunity to overcome PRR-mediated plant defence (de Jonge et al. 2012; Rovenich et al. 2014). Such effectors are often lineage-specific, facilitate colonization of the plant, and therefore contribute to virulence of the pathogen. In turn, plants evolved immune receptors, typically called resistance (R) proteins, which recognize particular effectors or their activities to mount an immune response known as effector-triggered immunity (ETI) (Jones and Dangl 2006). To overcome recognition by the plant, pathogens have to mutate or loose the recognized effector or, alternatively, evolve new effectors to suppress the ETI response. Nevertheless, not all pathogen-secreted molecules follow the strict MAMP-effector dichotomy but rather display traits of either class of molecules, and therefore MTI and ETI responses cannot strictly be separated (Thomma et al. 2011). Typical examples of such pathogen-secreted molecules are Nep1-like proteins, which are virulence factors that are dispersed throughout three kingdoms of life that act as a MAMP at least in Arabidopsis (Böhm et al. 2014; Oome et al. 2014). Although less widely distributed, phenomena have been observed for homologs of the Avr4 effector from the tomato leaf mould fungus Cladosporium fulvum (Stergiopoulos et al. 2010), and for homologs of the Ave1 effector from the vascular wilt fungus Verticillium dahliae (de Jonge et al. 2012). This has inspired the proposal of the so-called invasion model, which states that plants evolved receptors for all types of molecules, pathogen- as well as host-derived, that can betray microbial invasion to mount the appropriate immune responses (Cook et al. 2015).

V. dahliae is a soil-borne fungal pathogen that causes vascular wilt in over 200 plant species, including important crop species (Fradin and Thomma 2006). Resistance to $V$. dahliae is mediated by the immune receptor Ve1 (Fradin et al. 2009; Kawchuk et al. 2001), of which homologs were found in mint, hop and cotton amongst other plant species (Majer et al. 2014; Vining and Davis 2009; Zhang et al. 2011). Recently, the effector protein that is secreted by $V$. dahliae race 1 strains and that activates Ve1mediated resistance was identified as Ave1, and all resistance-breaking race 2 strains analysed thus far lack the complete Ave1 gene (de Jonge et al. 2012; Faino et al. 
2016). Importantly, Ave1 deletion strains show reduced aggressiveness on tomato plants that lack Ve1, revealing that Ave1 acts as a virulence factor on this host species. Additionally, Ave1 was also shown to act as a virulence factor of $V$. dahliae on Arabidopsis plants (de Jonge et al. 2012). However, how Ave1 contributes to V. dahliae virulence on these plant hosts remains unknown thus far.

Intriguingly, although pathogen effectors are typically lineage-specific traits, many homologs of Ave1 can be found in public databases (de Jonge et al. 2012). Remarkably, a wealth of Ave1 homologs was found in plants as well as in a handful of fungal plant pathogens, including Fusarium oxysporum (FoAve1), Cercospora beticola (CbAve1) and Colletotrichum higginsianum (ChAve1), and in the bacterial plant pathogen Xanthomonas axonopodis (XacPNP) (de Jonge et al. 2012; Gottig et al. 2008). Interestingly, it was shown that $V e 1$ is not confined to $V$. dahliae Ave 1 (VdAve1), as Ve1 is able to also recognize FoAve1 and CbAve1, while ChAve1 is not recognized (de Jonge et al. 2012). Consequently, Ve1 was indeed found to be able to recognize F. oxysporum in tomato and mount a defense response (de Jonge et al. 2012). Remarkably, Ve1 was furthermore found to recognize an endogenous Ave1 homolog (SIAve1) that shares a high degree of identity with VdAve1 (de Jonge et al. 2012).

Most plant homologs of Ave1 have been annotated either as plant natriuretic peptides (PNPs) or as expansin-like proteins, and functionally analyzed members were implicated in the regulation of water and ion homeostasis, and consequently in many downstream processes including growth, net water uptake, photosynthesis, stomatal opening and gas exchange (Gehring and Irving 2003; Ludidi et al. 2004; Maryani et al. 2001; Pharmawati et al. 2001). The observation that the microbialderived Ave 1 homologs do not follow the phylogeny of the species in which they occur is generally taken as evidence for horizontal acquisition, and it has thus been speculated that the microbial Ave1 homologs were acquired from plants (de Jonge et al. 2012; Gottig et al. 2008). In this respect it is interesting that the $X$. axonopodis homolog XacPNP affects homeostasis and photosynthesis in citrus plants, and thus promotes bacterial proliferation as a virulence factor in citrus plants (Garavaglia et al. 2010; Gottig et al. 2008; Nembaware et al. 2004). In this study, we investigate whether the Ave1 homologs of several fungal pathogens act as genuine virulence factors. 


\section{Results}

\section{Characterization of Vel-mediated tomato defence against Fusarium oxysporum}

We previously demonstrated that tomato Ve1 can recognize FoAve1, leading to a defence response that affects infection of Ve1-carrying tomato by F. oxysporum (de Jonge et al. 2012). Because recognition of FoAve1 by Ve1-carrying tomato plants does not lead to full immunity against F. oxysporum we investigated the occurrence of expression of FoAve1 in two F. oxysporum strains, Bt.01 and Fol4287, in tomato plants lacking Ve1 with reverse-transcription PCR at 14 dpi. Clear expression of FoAve1 was monitored at 14 dpi (Figure 1A).

A

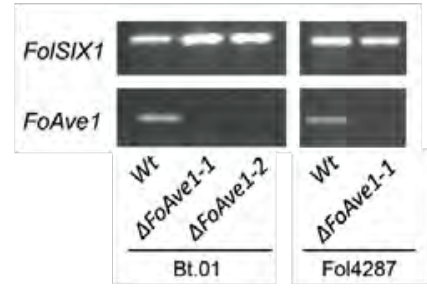

B

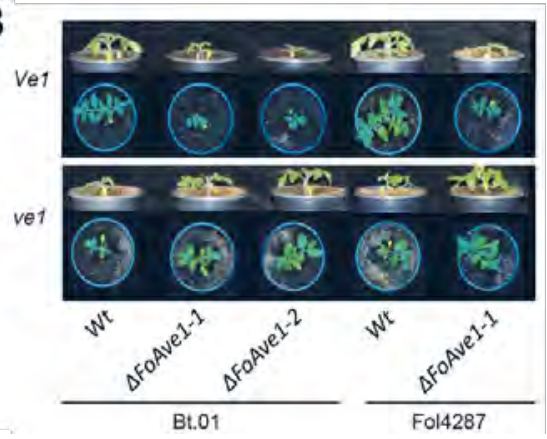

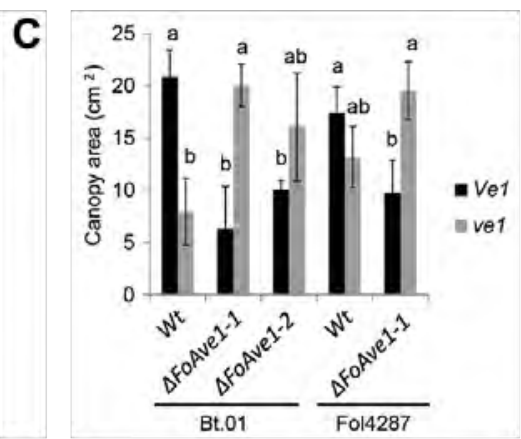

D

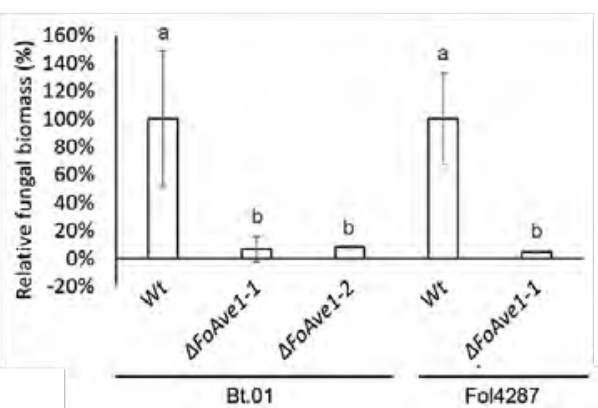

FIGURE 1 | FoAve1 is a virulence factor recognized by tomato Ve1. (A) Expression of FoAve1 in F. oxysporum in wild type $(\mathrm{Wt})$ strains Bt.01 and Fol4287 and the FoAve1 deletion strains ( $\triangle$ FoAve1) inoculated on tomato at $14 \mathrm{dpi}$. (B) (Upper) FoAve1 deletion strains ( $\triangle F o A v e 1)$ of F. oxysporum strains Bt.01 and Fol4287 escape recognition by tomato Ve1 compared with the corresponding wild type (Wt) strains evidenced by stunted Ve1 plants at 14 dpi. (Lower) FoAve1 deletion strains display reduced virulence compared with the corresponding $F$. oxysporum wild type strains Bt.01 and Fol4287 on tomato lacking Ve1 (ve1) evidenced by reduced stunting at 14 dpi. (C) Canopy area of Ve1 tomato and tomato lacking Ve1 after inoculation with FoAve1 deletion strains and the corresponding F. oxysporum wild type strains at $14 \mathrm{dpi}$. (D) FoAve1 deletion strains display reduced virulence compared to the corresponding F. oxysporum wild type strains Bt.01 and Fol4287 on tomato lacking Ve1 evidenced by reduced fungal biomass at 14 dpi. 8 plants were pooled per 2 plants $(n=4)$. Different letter labels indicate statistically significant differences $(P<0.05)$. Similar results were observed in at least 3 biological replications for all experiments. 
To provide further evidence for the role of FoAve1-recognition in Ve1-mediated tomato defence against Fusarium wilt, we generated FoAve1 deletion mutants in F. oxysporum strains Bt.01 and Fol4287 through homologous recombination. Deletion of FoAve1 was confirmed by PCR (Supplemental Figure 1A) and the deletion strains were subsequently evaluated for their ability to colonize Ve1 tomato plants. As anticipated, inoculation of Ve1 tomato plants with FoAve1 deletion strains resulted in more extensive symptoms of Fusarium wilt disease when compared to tomato plants inoculated with the corresponding wild type strains (Figure 1B (Upper) and 1C). Altogether, these data show that tomato Ve1 is able to recognize FoAve1 and activate a defense response that inhibits colonization by $F$. oxysporum, albeit that the response does not fully arrest the fungus and immunity is not fully established. At later stages after inoculation clear symptoms of wilt disease are observed on Ve1 plants.

\section{FoAve 1 is a virulence factor of $F$. oxysporum during tomato colonization}

According to the paradigm that plant immune receptors recognize crucial virulence factors of microbial pathogens, it is expected that FoAve1 contributes to $F$. oxysporum virulence. To test this hypothesis, FoAve1 deletion strains were inoculated on tomato plants lacking Ve1. Indeed, FoAve1 deletion strains displayed reduced virulence on tomato plants when compared to the corresponding $F$. oxysporum wild type strain as visualized by the difference in canopy area of the inoculated tomato plants (Figure 1B (Lower) and 1C). In addition, plants inoculated with FoAve1 deletion strains resulted in less fungal colonization compared to those inoculated with wild type $F$. oxysporum (Figure 1D). These results suggest that FoAve 1 is a virulence factor of $F$. oxysporum, albeit that its contribution to virulence appears to be relatively minor.

\section{ChAve 1 is not a virulence factor of $C$. higginsianum during Arabidopsis colonization}

Besides the vascular wilt fungi $V$. dahliae and F. oxysporum, that belong to the class of Sordariomycetes, an Ave1 homolog is found in the foliar Sordariomycete $C$. higginsianum (ChAve1). Using real-time PCR, ChAve1 expression was monitored in wild type $C$. higginsianum during colonization of Arabidopsis plants from 1 to 4 dpi, by which time the plant tissue was completely macerated. No expression of ChAve1 was detected at any of these time points. Nevertheless, we investigated whether ChAve1 contributes to virulence in this foliar pathogen. For this purpose, ChAve1 deletion strains were generated that, after confirmation with PCR (Supplemental Figure 1B), were evaluated for aggressiveness on Arabidopis thaliana. As expected ChAve1 deletion strains did not show reduced virulence when compared to the 
corresponding wild type $C$. higginsianum, as infections resulted in lesions with similar sizes (Figure 2A). Moreover, similar levels of pathogen biomass were recorded in plants inoculated with the wild-type fungus and the deletion strains (Figure 2B). Together these results show that ChAve 1 is not a virulence factor of $C$. higginsianum on Arabidopsis, likely due to the lack of ChAve1 expression.

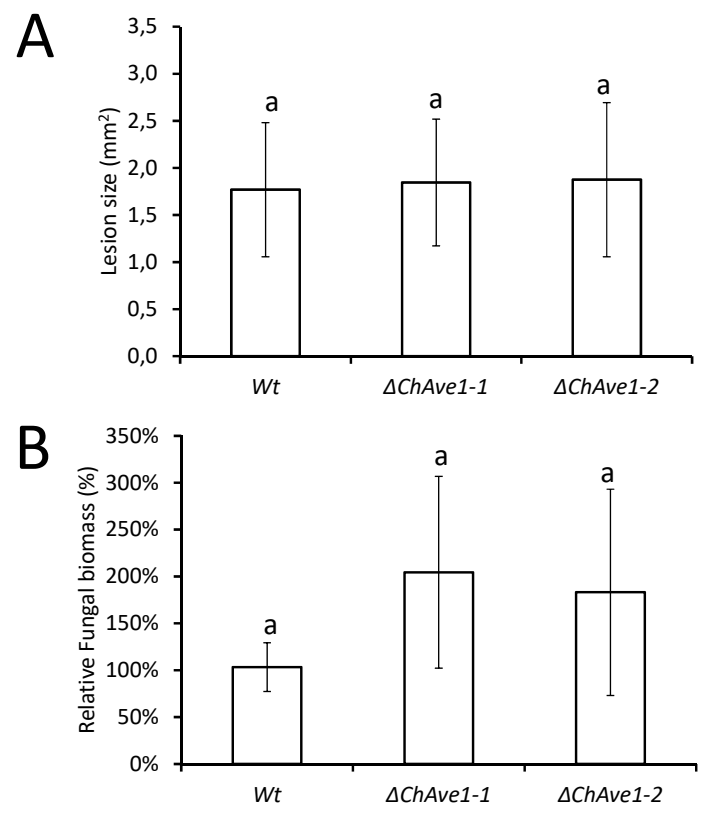

FIGURE 2 | ChAve1 is not a virulence factor of $\boldsymbol{C}$. higginsianum. (A) ChAve 1 deletion strains ( $\triangle$ ChAve 1$)$ show a similar lesion size compared to the corresponding $C$. higginsianum wild type strain (Wt) at 3 dpi on 3-weekold A. thaliana. Twenty four lesions on three $A$. thaliana plants were measured per strain. No significant difference was observed $(P<0.05)$. (B) No difference in fungal biomass was observed at 4 dpi on spray inoculated 3-week-old Arabidopsis plants $(n=4)$. Different letter labels indicate statistically significant differences $(P<0.05)$. Similar results were observed in at least 3 biological replications for all experiments in this figure.

\section{CbAve 1 is a virulence factor of $\mathrm{C}$. beticola during sugarbeet colonization}

Besides Sordariomycetes, Ave1 homologs were also identified as we previously identified an Ave1 homolog in the Dothidiomycete C. beticola (CbAve1). Expression of CbAve 1 was investigated in $C$. beticola during colonization of sugarbeet plants between 3 and 18 dpi using real-time PCR. Clear expression was detected between these time points. To evaluate the contribution of $C b A v e 1$ in $C$. beticola virulence, CbAve 1 deletion strains were generated and confirmed with PCR. For this pathogen, deletion of the Ave1 homolog resulted in reduced virulence compared to the corresponding wild type strain, as infection resulted in reduced numbers and size of the lesions (Figure 3A; B). As expected, CbAve1 deletion strains showed reduced colonization on sugarbeet plants compared to the corresponding $C$. beticola wild type strain (Figure $3 C$ ). Taken together, these results show that $\mathrm{CbAve} 1$ is a virulence factor of $C$. beticola. 
A

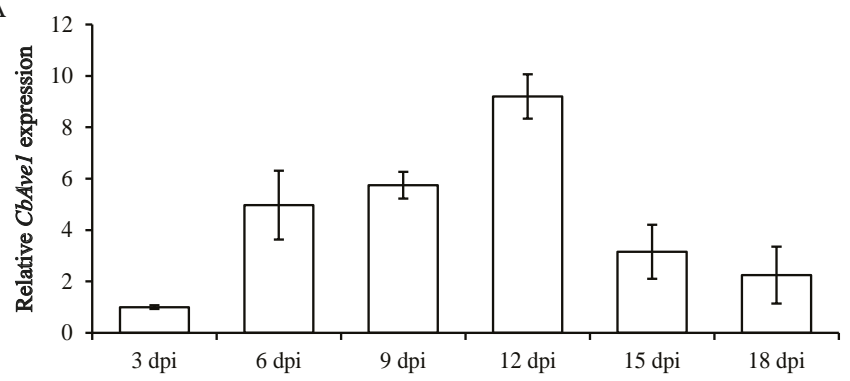

B

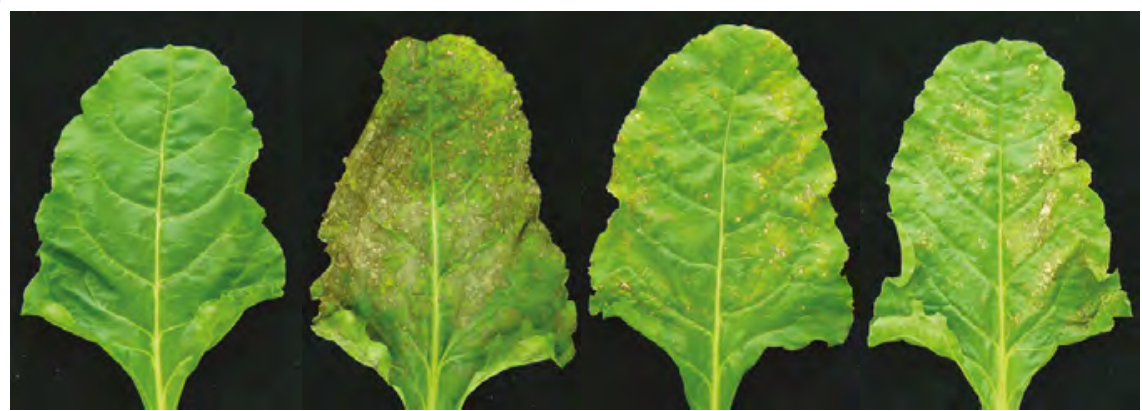

Mock

WT

$\triangle$ CbAvel \#1

$\triangle$ CbAvel \#2

Cercospora beticola

C

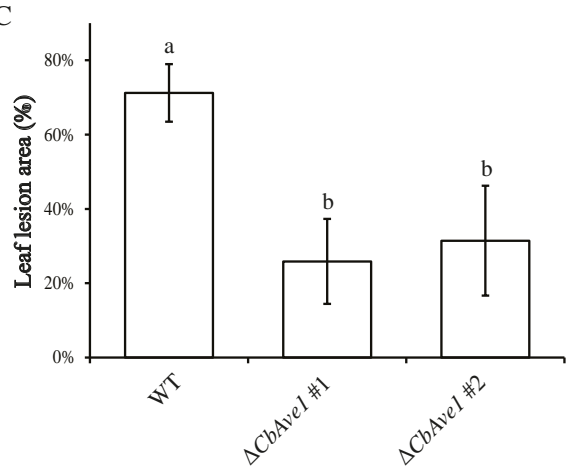

D

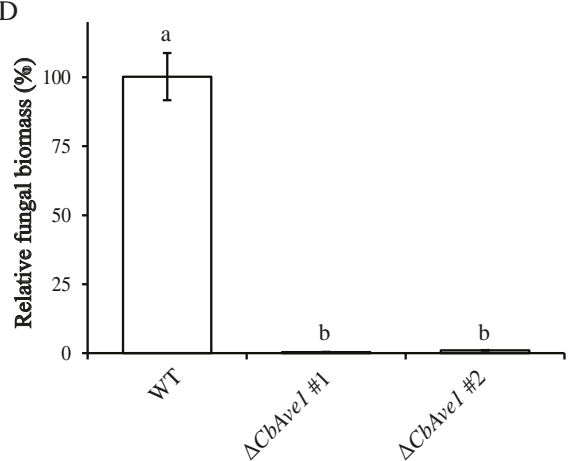

FIGURE 3 | CbAve1 is a virulence factor of $C$. beticola. (A) Expression of CbAve1 during infection of $C$. beticola on sugar beet. Leaves of 6-to 7-week-old sugar beet plants were inoculated with wild-type $C$. beticola and collected at regular intervals from 3 to 18 days post inoculation (dpi). RTqPCR was performed to determine the relative expression levels of CbAve1 using the $C$. beticola actin gene as a reference, and compared with CbAve1 expression in $C$. beticola in sugar beet plants upon the wild-type $C$. beticola inoculation at $3 \mathrm{dpi}$, which is set to 1. (B) CbAve1 deletion strains ( $\triangle C b A v e 1 \# 1$ and $\triangle C b A v e 1 \# 2$ ) show reduced virulence compared to the corresponding C. beticola wild type strain (WT) visualized by a reduction in lesion area on sugar beet leaves at 15 dpi. (C) Quantification of leaf lesion caused by C. beticola on sugar beet plants at $15 \mathrm{dpi}$ $(n>5)$. Bars represent the average percentage of leaf lesion area of whole leaf area with standard deviations. (D) Fungal biomass determined with QPCR in Cercospora-inoculated sugar beet plants at $15 \mathrm{dpi}$. The fungal biomass in sugar beet plants upon inoculation with the wild-type $C$. beticola is set to $100 \%$ (control). Different letter labels indicate statistically significant differences $(P<0.05)$. 


\section{Functional diversification among plant and fungal Ave 1 homologs}

VdAve1 is a virulence factor of $V$. dahliae that shows a high degree of identity with homologs from plants. It has therefore been proposed that $V d A v e 1$ has been acquired by $V$. dahliae from plants through horizontal gene transfer (de Jonge et al. 2012). However, the function of VdAve1 through which it contributes to $V$. dahliae aggressiveness remains presently enigmatic. Similarly, the function of the Ave1 homologs that are found in various pathogens also remains unclear. In order to evaluate whether all homologs share their functionality, we tested whether they can complement the virulence defect that is observed upon VdAve1 deletion in V. dahliae. To this end, we transformed one of the VdAve1 deletion strains of $V$. dahliae with constructs to drive expression of the plant homologs derived from tomato (S. lycopersicum; SIAve1) and grape (V. vinifera; VvAve1) by the $V$. dahliae VdAve1 promotor (de Jonge et al. 2012). To confirm that the plant homologs were expressed in $V$. dahliae upon colonization of tomato we monitored SIAve 1 and VvAve1 expression with PCR. At 14 dpi we monitored clear expression of SIAve1 and VvAve1 in 3 transformants for each construct (Supplemental Figure 2a). Subsequently, we evaluated the ability of these transformants to cause disease on tomato plants lacking Ve1. All VdAve1 deletion strains carrying SIAve1 and VvAve1 showed a similar phenotype and fungal colonization as the corresponding VdAve1 deletion strain (Figure 4; Supplemental Figure 2). These results suggest that two plant Ave1 homologs that share a high identity with VdAve1 are unable to restore virulence in a VdAve1 deletion strain, suggesting that Ave1 homologs from plants act in a different manner as VdAve1.

Subsequently, we evaluated whether microbial Ave1 homologs can reinstall the compromised virulence of $V$. dahliae that results from $V d A v e 1$ deletion. To this end, we transformed one of the VdAve1 deletion strains with constructs to drive expression of the Ave1 homologs derived from F. oxysporum (FoAve1) C. higginsianum (ChAve1) and C. beticola (CbAve1) by the VdAve1 promoter. We also included the Ave1 homolog derived from the plant pathogenic bacterium Xanthomonas axonopodis (XacPNP) that was previously described as a virulence factor (Gottig et al. 2008). We confirmed in a minimum of 2 transformants that the constructs were expressed (Supplemental Figure 2a). Subsequently, we tested the $V$. dahliae transformants on tomato lacking Ve1 and compared them with the corresponding wild type $V$. dahliae and the $V$. dahliae VdAve1 deletion strain complemented with VdAve1. VdAve1 deletion strains expressing FoAve1, CbAve1, ChAve1 and XacPNP showed a similar disease phenotype and fungal colonization as VdAve1 deletion strains (Figure 4; Supplemental Figure 2). (Figure 4; Supplemental Figure 2). These results show that the Ave1 homologs FoAve1, CbAve1, ChAve1 and XacPNP cannot restore virulence in a VdAve1 deletion strain which may suggest that Ave1 homologs of $V$. dahliae on the one hand, and F. oxysporum, C. beticola, $C$. higginsianum and $X$. axonopodis on the other hand, contribute to fungal virulence in a different manner. 


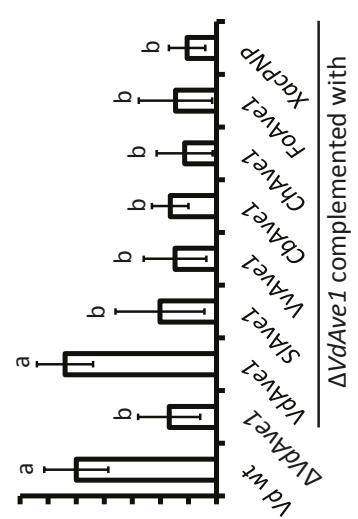

웍웡ㅇㅇㅇㅇㅇㅇ

$\cup \quad(\%)$ ssemo!̣ |esun

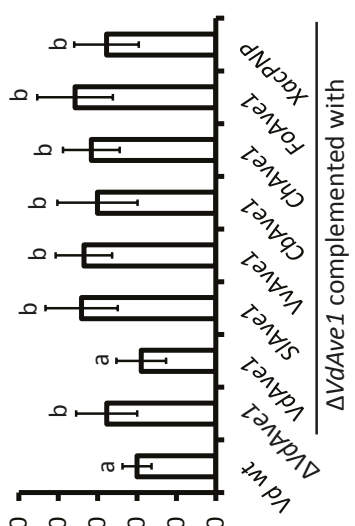

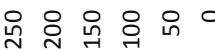

$\infty$

(\%) еaxe Кdoueว

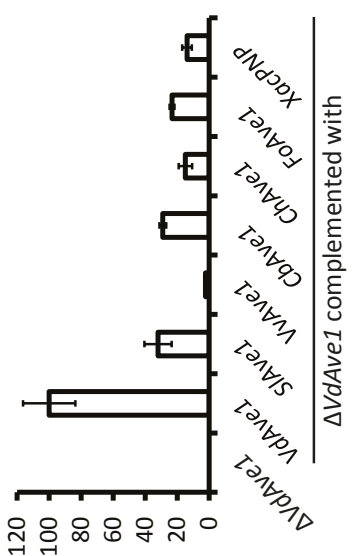

$\varangle$

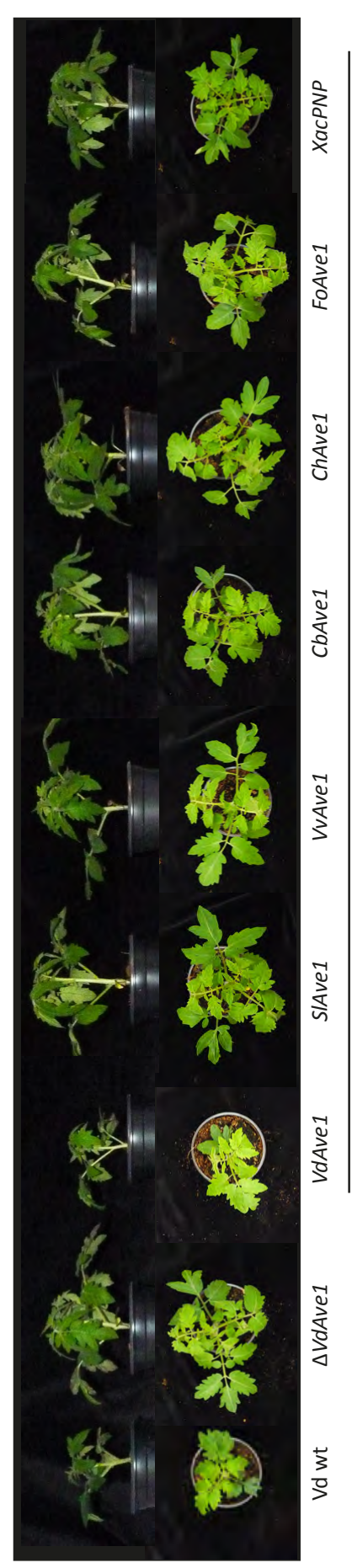

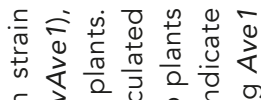

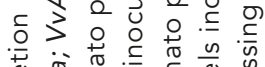

बै बै है है बै

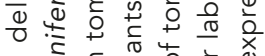

๘)

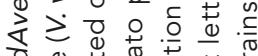

万人

ब

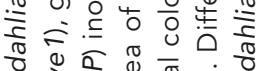

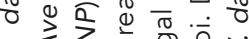

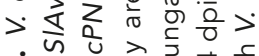

ن

ब.

는

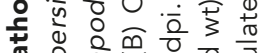

๘

प

$\pm \geq \times \frac{0}{0} \pm$

는 v

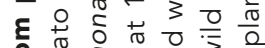

ᄂ $\varepsilon$ हो $\geq \geqslant$

ठ

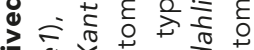

एक $x+0 \frac{\pi}{0}+$

d $\begin{aligned} & 0 \\ & 0\end{aligned}$

น

: $\underset{0}{0}$

일

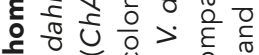

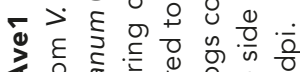

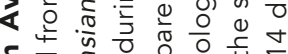

के О

๘

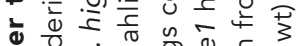

¿ $u$ 万

๙

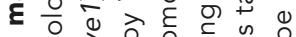

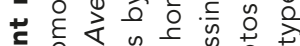

ब 00 \%

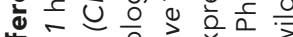

专

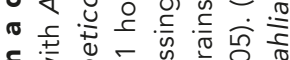

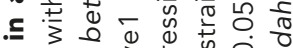

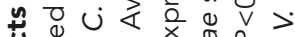

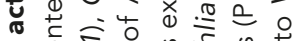

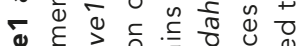

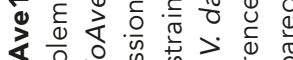

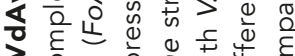

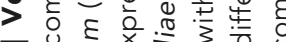

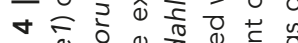

山

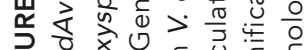

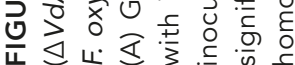




\section{Discussion}

\section{The role of FoAve 1 in recognition by tomato immune receptor $\mathrm{Vel}$}

We have previously shown that recognition of FoAve1 by Ve1 leads to a defence response (de Jonge et al. 2012) and that Ve1 tomato plants can recognize $F$. oxysporum. To confirm that FoAve1 is involved in recognition we first analysed FoAve1 expression. A previous study could not detect FoAve1 expression in F. oxysporum after inoculation on tomato (Schmidt et al. 2013). However, in the conditions that we tested clear FoAve 1 expression was monitored in F. oxysporum on tomato at 14 dpi. In addition, deletion of FoAve 1 resulted in abolishment of recognition by $\mathrm{Ve} 1$. Our data suggest that the incomplete disease resistance cannot be attributed to a lack of FoAve1 expression, but may be the result of a less efficient detection of this Ave1 homolog by Ve1. Similar differences in recognition efficiencies were previously observed for Cladosporium fulvum effector Ecp2 and its homolog MfEcp2 of Mycosphaerella fijiensis when coexpressed with tomato immune receptor Cf-Ecp2 (Stergiopoulos et al. 2010).

\section{Some Ave 1 homologs act as virulence factors}

Previously, we have shown that Ave 1 is a virulence factor of $V$. dahliae (de Jonge et al. 2012). In this study, we tested wheter Ave1 homologs in other plant pathogens act as virulence factors as well. We showed that deletion of Ave1 homologs in F. oxysporum and $C$. beticola resulted in reduced aggressiveness on their respective plant hosts. In contrast, the Ave1 homolog ChAve1 of the foliar pathogen C. higginsianum does not contribute to virulence due to the lack of expression in planta. Possibly, the lack of expression is due to the availability of effectors with a similar function that render ChAve1 functionality redundant. Alternatively, recognition of ChAve1 by a host immune receptor posed pressure on $C$. higginsianum to avoid recognition, resulting in the lack of ChAve1 expression. Interestingly, homologs of tomato Ve1 have been identified in many other plant species, of which some within as well as outside the Solanaceae family have been shown to be functional immune receptors (Song et al. 2017a).

\section{Functional diversification among Ave 1 homologs}

Since Ave1 homologs derived from plants display high similarity to Ave1, it has been suggested that Ave1 was acquired through horizontal gene transfer from plants (de Jonge et al. 2012). As the function of the Ave1 homologs remain unknown we tested whether the Ave1 homologs share the same functionality by complementing a $V$. dahiae Ave1 deletion strain with homologs derived from plants and various plant 
pathogens. Highly similar Ave1 homologs derived from plants are unable to restore virulence in an Ave1 deletion strain. Therefore we conclude that Ave1 homologs derived from plants act in a different manner as Ave1. Similarly, Ave1 homologs derived from F. oxysporum, C. beticola, C. higginsianum and X. axonopodis cannot restore the virulence penalty caused by the deletion of Ave1. Therefore, we conclude that Ave1 from V. dahliae on the one hand, and Ave1 homologs derived from F. oxysporum, C. beticola, C. higginsianum and $X$. axonopodis on the other hand, contribute to virulence in a different manner. Similarly, functional diversification within effector families has been observed for LysM and NLP effectors (Kombrink and Thomma 2013; Santhanam et al., 2013). Alternatively, it is important to note that $V$. dahliae, $C$. beticola, $C$. higginsianum and $X$. axonopodis have diverse plant hosts and that the reason for the inability to restore virulence in an $V$. dahliae Ave 1 deletion strain could be that each homolog targets a host-specific protein. This would for example explain why the homolog of $C$. beticola that likely targets a sugarbeet protein cannot restore virulence in an $\mathrm{V}$. dahliae Ave1 deletion strain colonizing tomato. Host specific effector adaptation has been suggested for the closely related oomycetes Phytophtora infestans and $P$. mirabilis that are pathogens on different hosts. Homologous effectors of both pathogens act more efficiently on their specific host target than on a "foreign" host target (Dong et al. 2014). Nevertheless, the finding that the Ave1 homolog from F. oxysporum f.sp. lycopersici cannot complement the VdAve1 deletion strain for virulence on tomato argues against this hypothesis.

\section{Materials and methods}

\section{Deletion and complementation strains}

Deletion strains were generated in F. oxysporum and C. higginsianum by amplifying $1,5 \mathrm{~kb}$ sequences (Supplemental table 1) flanking the coding sequence of FoAve1 and ChAve1, respectively, and cloned as previously described (Frandsen et al. 2008) into vector pRF-HU2 containing a nourseothricin cassette for selection. For the complementation of $V$. dahliae Ave1 deletion strains FoAve1, CbAve1, ChAve1, SIPNP, VvPNP and XacPNP were obtained by gene synthesis (Eurofins Genomics, Ebersberg, Germany). The synthesized genes were then cloned into vector pFBT005 under the VdAve1 promoter, containing a nourseothricin cassette for selection. F. oxysporum (Fol4287 and Bt.01) and C. higginsianum (IMI349063A) conidiospores were transformed on a Hybond- $\mathrm{N}^{+}$filter by $\mathrm{A}$. tumefaciens carrying the pRF-HU2 plasmid containing the constructs and a V. dahliae Ave1 deletion strain (JR2) was transformed by A. tumefaciens carrying the pFBT005 plasmid containing the constructs as previously described (Santhanam 2012). Transformants 
were then selected on potato dextrose agar (PDA) (Thermo Fisher Scientific Inc, Breda, The Netherlands) containing hygromycin B (Duchefa Biochemie BV, Haarlem, The Netherlands) for selection of F. oxysporum, C. beticola and C. higginsianum transformants or nourseothricin sulphate (Sigma Aldrich Chemie BV, Zwijndrecht, The Netherlands) for selection of $V$. dahliae transformants. After five to seven days at room temperature, individual transformants were transferred from the filter to fresh PDA plates with the appropriate selection and incubated for ten days (Santhanam 2012). To verify the transformants, fungal spores were collected and genomic DNA was extracted followed by a PCR to test the presence of the hygromycin or nourseothricin cassette and the presence of the construct in the genome (Supplemental table 1).

CbAve1 deletion mutants of $C$. beticola were generated using the split-marker approach described by Catlett et al. (2003). Genomic DNA of the wild type C. beticola strain 09-40 and pDAN vector (Friesen et al.,2006) served as PCR templates to generate split-marker PCR constructs used for transformation. Primers are listed in Supplemental table 1. PEG-mediated transformation of the wild type $C$. beticola strain 09-40 was performed as previously described (Bolton et al.,2016). Sitedirected gene replacement was confirmed by the absence of PCR product using split-marker CbAve1 1F forward primer of the $5^{\prime}$ flank of the target gene and MDB760 reverse primer designed on the coding sequence of CbAve1.

\section{Pathogen inoculations}

Plants were grown in soil in the greenhouse at $21^{\circ} \mathrm{C} / 19^{\circ} \mathrm{C}$ during $16-\mathrm{h} / 8-\mathrm{h}$ day/night periods, respectively, with $70 \%$ relative humidity and $100 \mathrm{~W} \mathrm{~m}^{-2}$ supplemental light when the intensity dropped below $150 \mathrm{~W} \mathrm{~m}^{-2}$.

For V. dahliae and F. oxysporum inoculations, 10-day-old tomato (Solanum lycopersicum; cv. MoneyMaker or MoneyMaker 35S:Ve1) (Fradin et al. 2009) were uprooted, rinsed in water and dipped for 5 minutes in a suspension of $10^{6}$ conidiospores per $\mathrm{mL}$ of water harvested from 1- to 2-week-old V. dahliae or F. oxysporum cultures on PDA as previously described (Fradin et al. 2009). Control plants received the same treatment, but the roots were dipped in water without conidiospores. After replanting in fresh soil, plants were incubated at standard greenhouse conditions. Disease development was monitored up to 21 days post inoculation (dpi).

For C. higginsianum inoculations 3-week-old Arabidopsis (A. thaliana ecotype Co-0 or Col-0 35S:Ve1) (Fradin et al. 2011) were inoculated on the leaves with either 2 $\mu \mathrm{l}$ drops or sprayed with a suspension of $10^{6}$ conidia per $\mathrm{mL}$ of water harvested from 1- to 2-week-old C. higginsianum cultures on Mathurs' agar as previously 
described (O'Connell et al. 2004; Sherriff et al. 1994). Control plants received the same treatment, but the leaves were sprayed with water without conidiospores. After sealing the plants inside a transparent closed box lined with wet tissue paper to provide high humidity, they were incubated at $25^{\circ} \mathrm{C}$, under a 16 -h/ 8-h light/dark regime (O'Connell et al. 2004). Disease development was monitored up to $4 \mathrm{dpi}$.

For C. beticola inoculations 6- to 7-week-old sugarbeet plants (Beta vulgaris) were inoculated by evenly spaying spore suspension of $10^{5}$ conidia per $\mathrm{mL}$ of water on the lower side of the leaves harvested from $C$. beticola cultures growing on V8 solid medium as previously described (Bolton et al. 2012). Control plants received the same treatment, but the leaves were sprayed with water. Plants were incubated at standard greenhouse conditions and disease development was monitored up to $18 \mathrm{dpi}$.

\section{Fungal biomass and gene expression}

Stem sections of tomato, cut from the base of the stem up to the cotyledons, were collected at 7 and 14 dpi from plant inoculated with $F$. oxysporum or V. dahliae. Arabidopsis leaves were collected at 1-4 dpi after inoculation with $C$. higginsianum. Sugarbeet leaves were collected at regular intervals between 3 and 18 dpi from plants inoculated with $C$. beticola. Collected plant tissue was flash frozen in liquid nitrogen and ground to powder, of which an aliquot of $\sim 100 \mathrm{mg}$ was used for RNA extraction with the Quick-RNA ${ }^{\mathrm{TM}}$ Miniprep kit (Zymo Research Europe GmbH, Freiburg, Germany), and cDNA was synthesized using M-MLV Reverse Transcriptase (Promega Benelux BV, Leiden, The Netherlands). Fungal biomass was determined by quantifying the expression of VdGAPDH, FOTUB, ChTUB and CbAct relative to the expression of $S I R \cup B$ (tomato), AtRub (Arabidopsis), BvAct (sugarbeet), respectively. Expression of VdAve1, FoAve1, CbAve1, ChAve1, SIPNP and VvPNP was determined by quantifying the expression relative to the expression of VdGAPDH, FoTUB, ChTUB and CbAct, respectively (Supplemental table 1). Real-time PCR was carried out on an ABI7300 PCR machine (Applied Biosystems, Life Technologies Europe BV, Bleiswijk, The Netherlands) in combination with the qPCR SensiMix kit (BioLine, GC Biotech BV, Alphen aan den Rijn, The Netherlands). The following Real-time PCR conditions were used: an initial $95^{\circ} \mathrm{C}$ denaturation step for 10 minutes followed by denaturation for 15 seconds at $95^{\circ} \mathrm{C}$, annealing for 60 seconds at $60^{\circ} \mathrm{C}$, and extension at $72^{\circ} \mathrm{C}$ for 40 cycles.

\section{Acknowledgements}

Work in the laboratory of BPHJT is supported by the Research Council Earth and Life Sciences (ALW) of the Netherlands Organization for Scientific Research (NWO). We thank Bert Essenstam and Pauline Sanderson of Unifarm for excellent plant care. We thank Jelle Postma, Dorien Oppelaar and Saskia Bosman for technical support. 


\section{References}

Böhm, H., Albert, I., Oome, S., Raaymakers, T. M., Van den Ackerveken, G., and Nürnberger, T. 2014. A conserved peptide pattern from a widespread microbial virulence factor triggers pattern-induced immunity in Arabidopsis. PLoS Pathog 10:e1004491.

Boller, T., and Felix, G. 2009. A renaissance of elicitors: perception of microbe-associated molecular patterns and danger signals by pattern-recognition receptors. Annu Rev Plant Biol 60:379-406.

Bolton, M. D., Birla, K., Rivera-Varas, V., Rudolph, K. D., and Secor, G. A. 2012. Characterization of CbCyp51 from field isolates of Cercospora beticola. Phytopathology 102:298-305.

Cook, D. E., Mesarich, C. H., and Thomma, B. P. H. J. 2015. Understanding plant immunity as a surveillance system to detect invasion. Annu Rev Phytopathol.

de Jonge, R., van Esse, H. P., Maruthachalam, K., Bolton, M. D., Santhanam, P., Saber, M. K., Zhang, Z., Usami, T., Lievens, B., Subbarao, K. V., and Thomma, B. P. H. J. 2012. Tomato immune receptor Ve1 recognizes effector of multiple fungal pathogens uncovered by genome and RNA sequencing. Proc Natl Acad Sci U S A 109:5110-5115.

Dodds, P. N., and Rathjen, J. P. 2010. Plant immunity: towards an integrated view of plant-pathogen interactions. Nat Rev Genet 11:539-548.

Dong, S., Stam, R., Cano, L. M., Song, J., Sklenar, J., Yoshida, K., Bozkurt, T. O., Oliva, R., Liu, Z., Tian, M., Win, J., Banfield, M. J., Jones, A. M., van der Hoorn, R. A., and Kamoun, S. 2014. Effector specialization in a lineage of the Irish potato famine pathogen. Science 343:552-555.

Faino, L., Seidl, M. F., Shi-Kunne, X., Pauper, M., van den Berg, G. C., Wittenberg, A. H., and Thomma, B. P. 2016. Transposons passively and actively contribute to evolution of the two-speed genome of a fungal pathogen. Genome Res 26:1091-1100.

Fradin, E. F., and Thomma, B. P. H. J. 2006. Physiology and molecular aspects of Verticillium wilt diseases caused by V. dahliae and V. albo-atrum. Mol Plant Pathol 7:71-86.

Fradin, E. F., Abd-El-Haliem, A., Masini, L., van den Berg, G. C., Joosten, M. H., and Thomma, B. P. H. J. 2011. Interfamily transfer of tomato Ve1 mediates Verticillium resistance in Arabidopsis. Plant Physiol 156:2255-2265.

Fradin, E. F., Zhang, Z., Ayala, J. C. J., Castroverde, C. D., Nazar, R. N., Robb, J., Liu, C.-M., and Thomma, B. P. 2009. Genetic dissection of Verticillium wilt resistance mediated by tomato Ve1. Plant Physiology 150:320-332.

Frandsen, R. J., Andersson, J. A., Kristensen, M. B., and Giese, H. 2008. Efficient four fragment cloning for the construction of vectors for targeted gene replacement in filamentous fungi. BMC Mol Biol 9:70.

Garavaglia, B. S., Thomas, L., Gottig, N., Dunger, G., Garofalo, C. G., Daurelio, L. D., Ndimba, B., Orellano, E. G., Gehring, C., and Ottado, J. 2010. A eukaryotic-acquired gene by a biotrophic phytopathogen allows prolonged survival on the host by counteracting the shut-down of plant photosynthesis. PLoS One 5:e8950.

Gehring, C., and Irving, H. 2003. Natriuretic peptides - a class of heterologous molecules in plants. Int J Biochem Cell Biol 35:1318-1322.

Gottig, N., Garavaglia, B. S., Daurelio, L. D., Valentine, A., Gehring, C., Orellano, E. G., and Ottado, J. 2008. Xanthomonas axonopodis pv. citri uses a plant natriuretic peptide-like protein to modify host homeostasis. Proc Natl Acad Sci U S A 105:18631-18636.

Jones, J. D., and Dangl, J. L. 2006. The plant immune system. Nature 444:323-329.

Kawchuk, L. M., Hachey, J., Lynch, D. R., Kulcsar, F., Van Rooijen, G., Waterer, D. R., Robertson, A., Kokko, E., Byers, R., and Howard, R. J. 2001. Tomato Ve disease resistance genes encode cell surfacelike receptors. Proceedings of the National Academy of Sciences of the United States of America 98:6511-6515.

Ludidi, N., Morse, M., Sayed, M., Wherrett, T., Shabala, S., and Gehring, C. 2004. A recombinant plant natriuretic peptide causes rapid and spatially differentiated $\mathrm{K}+, \mathrm{Na}+$ and $\mathrm{H}+$ flux changes in Arabidopsis thaliana roots. Plant Cell Physiol 45:1093-1098. 
Majer, A., Javornik, B., Cerenak, A., and Jakse, J. 2014. Development of novel EST-derived resistance gene markers in hop (Humulus lupulus L.). Mol Breed 33:61-74.

Maryani, M., Bradley, G., Cahill, D., and Gehring, C. 2001. Natriuretic peptides and immunoreactants modify osmoticum-dependent volume changes in Solanum tuberosum L. mesophyll cell protoplasts. Plant Sci 161:443-452.

Nembaware, V., Seoighe, C., Sayed, M., and Gehring, C. 2004. A plant natriuretic peptide-like gene in the bacterial pathogen Xanthomonas axonopodis may induce hyper-hydration in the plant host: a hypothesis of molecular mimicry. BMC Evol Biol 4:10.

O'Connell, R., Herbert, C., Sreenivasaprasad, S., Khatib, M., Esquerré-Tugayé, M.-T., and Dumas, B. 2004. A novel Arabidopsis-Colletotrichum pathosystem for the molecular dissection of plant-fungal interactions. Mol. Plant Microbe Interact. 17:272-282.

Oome, S., Raaymakers, T. M., Cabral, A., Samwel, S., Böhm, H., Albert, I., Nürnberger, T., and Van den Ackerveken, G. 2014. Nep1-like proteins from three kingdoms of life act as a microbe-associated molecular pattern in Arabidopsis. Proc Natl Acad Sci U S A 111:16955-16960.

Pharmawati, M., Maryani, M. M., Nikolakopoulos, T., Gehring, C. A., and Irving, H. R. 2001. Cyclic GMP modulates stomatal opening induced by natriuretic peptides and immunoreactive analogues. Plant Physiol Biochem 39:385-394.

Rovenich, H., Boshoven, J. C., and Thomma, B. P. H. J. 2014. Filamentous pathogen effector functions: of pathogens, hosts and microbiomes. Curr Opin Plant Biol 20:96-103.

Santhanam, P. 2012. Random insertional mutagenesis in fungal genomes to identify virulence factors. Pages 509-517 in: Plant fungal pathogens. Methods in molecular biology, vol. 835. T. B. Bolton MD, ed. Humana press, Totowa.

Schmidt, S. M., Houterman, P. M., Schreiver, I., Ma, L., Amyotte, S., Chellappan, B., Boeren, S., Takken, F. L., and Rep, M. 2013. MITEs in the promoters of effector genes allow prediction of novel virulence genes in Fusarium oxysporum. BMC Genomics 14:119.

Sherriff, C., Whelan, M. J., Arnold, G. M., Lafay, J.-F., Brygoo, Y., and Bailey, J. A. 1994. Ribosomal DNA sequence analysis reveals new species groupings in the genus Colletotrichum. Exp Mycol 18:121138.

Song, Y., Zhang, Z., Seidl, M. F., Majer, A., Jakse, J., Javornik, B., and Thomma, B. P. 2017a. Broad taxonomic characterization of Verticillium wilt resistance genes reveals ancient origin of the tomato Ve1 immune receptor. Mol Plant Pathol 18:195-209.

Stergiopoulos, I., van den Burg, H. A., Ökmen, B., Beenen, H. G., van Liere, S., Kema, G. H., and de Wit, P. J. G. M. 2010. Tomato Cf resistance proteins mediate recognition of cognate homologous effectors from fungi pathogenic on dicots and monocots. Proc Natl Acad Sci U S A 107:7610-7615.

Thomma, B. P. H. J., Nurnberger, T., and Joosten, M. H. A. J. 2011. Of PAMPs and effectors: The blurred PTI-ETI dichotomy. Plant Cell 23:4-15.

Vining, K., and Davis, T. 2009. Isolation of a Ve homolog, mVe1, and its relationship to Verticillium wilt resistance in Mentha longifolia (L.) Huds. Mol Genet Genomics 282:173-184.

Zhang, Y., Wang, X., Yang, S., Chi, J., Zhang, G., and Ma, Z. 2011. Cloning and characterization of a Verticillium wilt resistance gene from Gossypium barbadense and functional analysis in Arabidopsis thaliana. Plant Cell Rep 30:2085-2096. 


\section{Supplemental data}

SUPPLEMENTAL TABLE 1 | Primers used in this study

\begin{tabular}{|c|c|c|}
\hline Primer & Sequence $\left(5^{\prime}-3^{\prime}\right)$ & Notes \\
\hline SIRub_OPCR_F & GAACAGTTTCTCACTGTTGAC & S. lycopersicum Rubisco \\
\hline SIRub_QPCR_R & CGTGAGAACCATAAGTCACC & S. Iycopersicum Rubisco \\
\hline FolSIX1 F & GTCTCACGAGCCAAGTCTACC & F. oxysporum Six1 \\
\hline FolSIX1_R & GAACCGCAGCCTCTTGAGCAT & F. oxysporum Six1 \\
\hline FolTub_F & CTCTGGCAACAAGTATGTTCCC & F. oxysporum Tubulin \\
\hline FolTub_R & TTGTCGGGACGGAAGAGCTGA & F. oxysporum Tubulin \\
\hline FolAve1_QPCR_F3 & ATATCGGAACTGCAAATATTCTCAAC & F. oxysporum Ave1 \\
\hline FolAve1_QPCR_R3 & CTTATACATTTCATCGTATACAGTCTGC & F. oxysporum Ave1 \\
\hline AtRub_QPCR_F & GCAAGTGTTGGGTTCAAAGCTGGTG & A. thaliana Rubisco \\
\hline AtRub_OPCR_R & CCAGGTTGAGGAGTTACTCGGAATGCTG & A. thaliana Rubisco \\
\hline ChELF1a_F & CTGGTACAAGGGTTGGGAGA & $\begin{array}{l}\text { C. higginsianum Elongation } \\
\text { factor }\end{array}$ \\
\hline ChELF1a_R & ACCGCCGATCTTGTAGACAT & $\begin{array}{l}\text { C. higginsianum Elongation } \\
\text { factor }\end{array}$ \\
\hline ChAve1_QPCR_F5 & CAAGATGCTATGGCAACAATATGAAC & C. higginsianum Ave1 \\
\hline ChAve1_QPCR_R5 & GTCTTGAGGAAAATCTATCGTATTTCTG & C. higginsianum Ave1 \\
\hline BvAct_OPCR_F & GATTTGGCACCACACCTTCT & B. vulgaris actin \\
\hline BvAct OPCR_R & TCTTTTCCCTGTTTGCCTTG & B. vulgaris actin \\
\hline CbAct_QPCR_F & ACATGGCTGGTCGTGATTTG & C. beticola actin \\
\hline CbAct_QPCR_F & TGTCCGTCAGGAAGCTCGTA & C. beticola actin \\
\hline CbAve1_OPCR F & ATTCCCTTCAGGCAACCTCT & C. beticola Ave1 \\
\hline CbAve1_OPCR R & CGGACAAGCTTCGCAATAAT & C. beticola Ave1 \\
\hline VdGapdh $F$ & CGAGTCCACTGGTGTCTTCA & V. dahliae GAPDH \\
\hline VdGapdh_R & CCCTCAACGATGGTGAACTT & V. dahliae GAPDH \\
\hline VdAve1-Fw5 & ATCCTACTATAACCCACCCTACCTTC & V. dahliae Ave1 \\
\hline VdAve1-Rv5 & CATCATATGAGTCCTGAGATAAGATCA & V. dahliae Ave1 \\
\hline XacAve1_QPCR_F & GCAATCGGTTTGCTCTTTTC & X. axonopodis XacPNP \\
\hline XacAve1_QPCR_R & AGCACCGTTATCCCACAGAC & X. axonopodis XacPNP \\
\hline VvAve1-OPCR-F2 & CGTTATAGGTTAAGGTGCCTGAGT & V. vinifera Ave1 \\
\hline VvAve1-OPCR-R2 & TTCATGCTAGGAGAGTGTGAAATG & V. vinifera Ave1 \\
\hline SIAve1_QPCR_F & CGTCGGGGAATCTATTTGTG & S. lycopersicum Ave1 \\
\hline SIAve1_OPCR_R & AAAGCATCCGTTGACAAAGC & S. lycopersicum Ave1 \\
\hline FolAve1_LB_F3 & GGTCTTAAUAACCTAACCTGTATCTAGACCAGAGTGTCT & Left border FolAve1 \\
\hline FolAve1_LB_R3 & GGCATTAAUCAGCAGGTGTTTAGGTATTGTTAAGATAAG & Left border FolAve1 \\
\hline FolAve1_RB F & GGACTTAAUGTTCTTAGAGCTTAAGGTCAATGTAGC & Right border FolAve1 \\
\hline FolAve1_RB_R & GGGTTTAAUCTGCAGCTTTCACGGGGCTA & Right border FolAve1 \\
\hline ChAve1_LBF & GGTCTTAAUGGCAGGAGGTCTAGGTGAGA & Left border ChAve1 \\
\hline ChAve1_LB R & GGCATTAAUCGACTTGAGTTTTGCTGCAC & Left border ChAve1 \\
\hline ChAve1_RB F & GGACTTAAUAAAGATTTTTGCAGTGCTTCA & Right border ChAve1 \\
\hline ChAve1_RB_R & GGGTTTAAUAGGCTGCAGAATGAGTTTCG & Right border ChAve1 \\
\hline pRF-HU2_Hyg_Fw & СTATTCCTTTGCCCTCGGACGAGTGC & Hygromycin primers \\
\hline pRF-HU2_Hyg_Rv & CGATGTAGGAGGGCGTGGATATGTCC & Hygromycin primers \\
\hline NAT_Fw & CGGGCCGGATTGGTCAAGATTTGC & Nourseothricin primers \\
\hline NAT_Rv & CGATTCGTCGTCCGATTCGTCG & Nourseothricin primers \\
\hline
\end{tabular}




\begin{tabular}{lll}
\hline Primer & Sequence (5' $^{\prime}$ 3') $^{\prime}$ & Notes \\
\hline MDB-277 & GACGTTGTAAAACGACGGCCAGTG & Split-marker HYG-F \\
MDB-258 & GGATGCCTCCGCTCGAAGTA & Split-marker HY-R \\
\hline MDB-259 & CGTTGCAAGACCTGCCTGAA & Split-marker YG-F \\
MDB-278 & CACAGGAAACAGCTATGACCATGA & Split-marker HYG-R \\
\hline MDB-1145 & GGCAGGTAGATGACGACCAT & HYG R2 \\
\hline CbAve1 1F & CAAGATTGGGCCTTCGTATG & CbAve1 split-marker left \\
CbAve1 2R & CACTGGCCGTCGTTTTACAACGTCTCTTCAATGGATCC & CbAve1 split-marker left \\
& GGACTG & border \\
CbAve1 3F & ICATGGTCATAGCTGTTCCTGTGTGAGTGGGGTTTT & CbAve1 split-marker right \\
& GGTTTC & border \\
\hline CbAve1 4R & AAGCATACCTCTTCGGCAAA & CbAve1 split-marker right \\
\hline MDB-760 & CAACGTCTGCCACAAGCTGCT & CbAve1 reverse \\
\hline
\end{tabular}

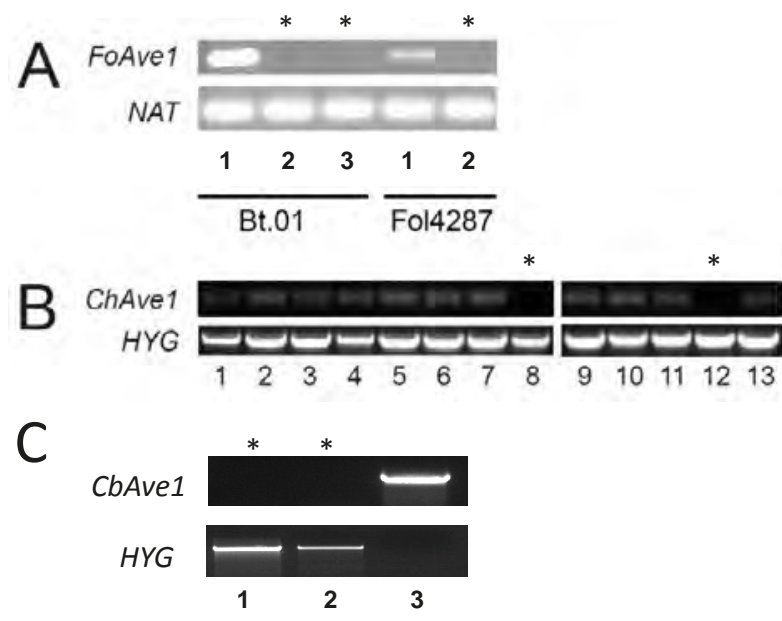

SUPPLEMENTAL FIGURE 1 | Verification of deletion strains. (A) Identification of $F$. oxysporum FoAve1 deletion strains by amplification of FoAve1 and selection marker nourseothricin (NAT) from DNA obtained from transformants. DNA bands were observed th the expected size. Asterisks indicate positive transformants, while transformants that lack an asterisk are ectopic transformants. (B) Identification of C. higginsianum ChAve1 deletion strains by amplification of ChAve1 and selection marker hygromycin (HYG) from DNA obtained from C. higginsianum transformants. DNA bands were observed at the expected size. Asterisks indicate positive transformants, while transformants that lack an asterisk are ectopic transformants. (C) Verification of CbAve1 deletion strains (1 and 2) based on absence of CbAve1 using gene-specific primers and presence of the hygromycin resistance cassette. Amplification on C. beticola Wt gDNA (3) as a control showing the presence of $\mathrm{CbAve} 1$ and absence of the hygromycin resistance cassette at the expected size. Asterisks indicate positive transformants. 


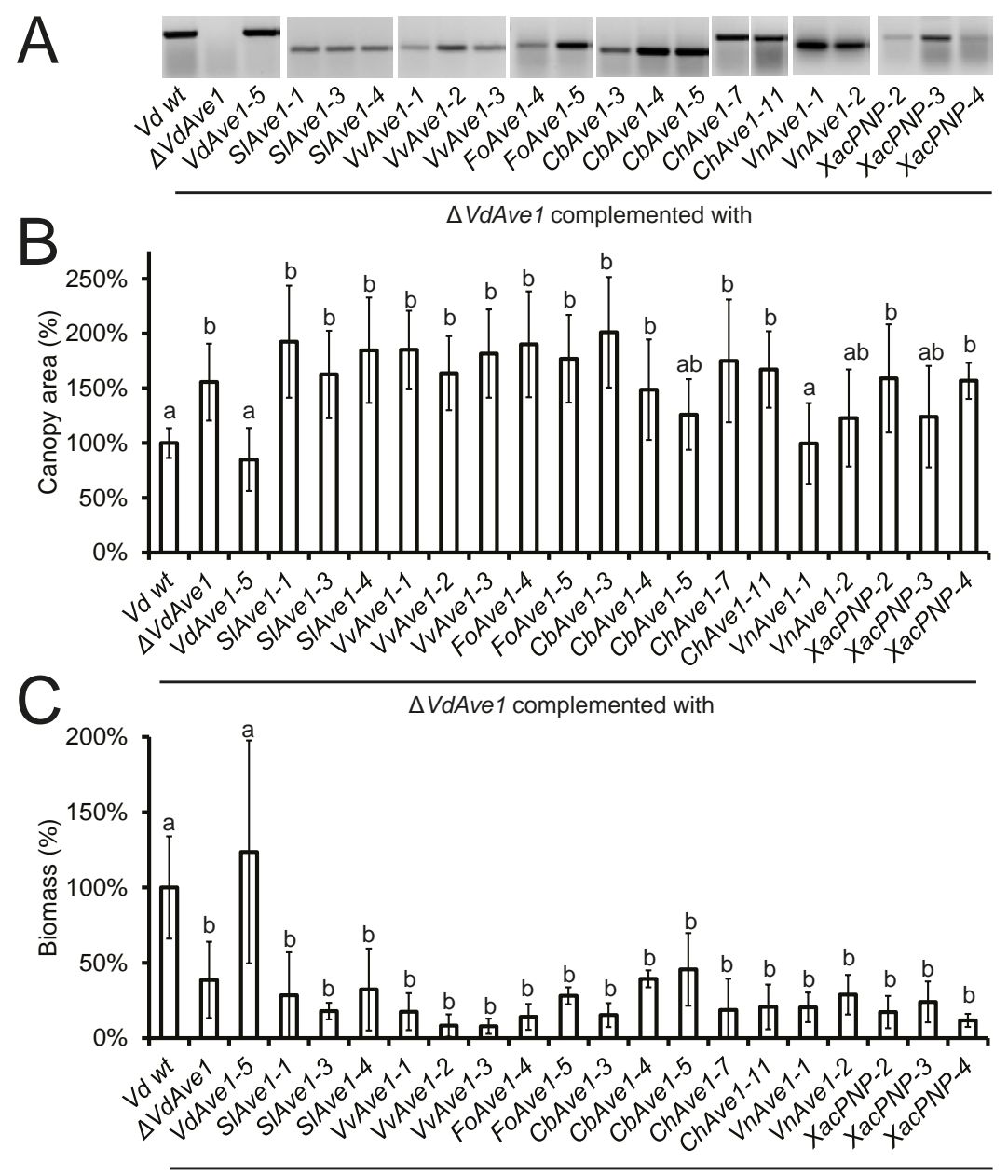

$\Delta$ VdAvel complemented with

SUPPLEMENTAL FIGURE 2 | VdAve1 acts in a different manner than Ave1 homologs derived from plant pathogens. $V$. dahliae $V d A v e 1$ deletion strain $(\triangle V d A v e 1)$ complemented with Ave1 homologs derived from $V$. dahliae (VdAve1), tomato (S. lycopersicum; SIAve1), grape (V. vinifera; VvAve1), F. oxysporum (FoAve1), C. beticola (CbAve1), C. higginsianum (ChAve1) and Xanthomonas axonopodis (XacPNP) inoculated on tomato plants. (A) Expression of VdAve1, SIAve1, VvAve1, FoAve1, CbAve1, ChAve1 and XacPNP in a minimum of 2 transformants. (B) Canopy area of tomato plants inoculated with $V$. dahliae strains expressing Ave1 homologs in a minimum of 2 transformants compared to $V$. dahliae wild type (Vd wt) at 14 dpi. (C) Fungal colonization of tomato plants inoculated with $V$. dahliae strains expressing Ave1 homologs compared to $V$. dahliae wild type ( $V d$ wt) at 14 dpi. Different letter labels indicate significant differences $(P<0.05)$. (D) Photos taken from the side and the top of tomato plants inoculated with $V$. dahliae strains expressing Ave 1 homologs compared to $V$. dahliae wild type $(V d w t)$ at $14 \mathrm{dpi}$. 



\title{
A nine amino acid peptide of Verticillium dahliae effector Ave 1 is required but not sufficient for recognition by tomato immune receptor Vel
}

\author{
Zhao Zhang" \\ Yin Song\# \\ Jordi C. Boshoven\# \\ Hanna Rovenich \\ Michael F. Seidl \\ Jernej Jakše \\ Karunakaran Maruthachalam \\ Chun-Ming Liu \\ Krishna V. Subbarao \\ Branka Javornik \\ Bart P.H.J. Thomma \\ \#These authors contributed equally
}

A modified version is available online at bioRxiv preprint server doi: https://doi.org/10.1101/103473 
Effectors are molecules secreted by plant pathogens to facilitate infection, often through deregulation of plant immune responses. During infection, race 1 strains of the soil borne vascular wilt fungus Verticillium dahliae secrete the effector protein Ave1 that is recognized by tomato immune receptor Ve1. Homologs of V. dahliae Ave1 (VdAve1), have been identified from the bacterial pathogen Xanthomonas axonopodis pv. citri (XacPNP), and in the fungal pathogens Fusarium oxysporum (FoAve1), Colletotrichum higginsianum (ChAve1) and Cercospora beticola (CbAve1). In this study, we identified a novel Ave1 homolog from $V$. nubilum (VnAve1), which is mainly known as saprophyte and opportunistic pathogen. The Ave1 homologs are differentially recognized by $V e 1$, with the most efficient recognition of VdAve1 and VnAve1, and little to no recognition of ChAve1 and XacPNP. Interestingly, C-terminal GFP-tagging of VdAve1 resulted in loss of recognition by $\mathrm{Ve} 1$, suggesting that exposure of the $\mathrm{C}$-terminus of VdAve1 is essential for recognition by Ve1. Truncations of VdAve1 and domain swaps between $\mathrm{VdAve} 1$ and XacPNP narrowed down the sequence that is required for $\mathrm{Ve} 1$ recognition to the $\mathrm{C}$-terminal nine amino acids. Site-directed mutagenesis was performed to analyse the contribution of individual amino acid residues to recognition by Ve1, revealing the importance of five amino acids from Val127 to Phe131. Nevertheless, fusions of the VdAve1 C-terminal nine amino acids to GFP or the Cladosporium fulvum effector Avr9 showed that this sequence is not sufficient to activate Ve1-mediated immunity. Finally, with a complementation experiment in $V$. dahliae we confirmed that the $\mathrm{C}$-terminal nine amino acids of Ave1 are required but not sufficient to activate Ve1-mediated immunity. 


\section{Introduction}

In nature, plants are threatened by diverse groups of potential microbial pathogens. However, only few of these potential pathogens actually cause disease, as plants have developed innate immunity to protect themselves against microbial attack (Dodds and Rathjen 2010; Thomma et al. 2011). In its simplest form, plant immunity against pathogen attack is governed by immune receptors that sense pathogenderived ligands to activate defense. Originally, the interaction between plant immune receptors and pathogen ligands was described in the "gene-for-gene" model, stating that the products of plant resistance $(R)$ genes induce race-specific resistance upon recognition of the products of corresponding pathogen avirulence (Avr) genes (Flor 1971). Decades later, an updated view of plant innate immunity has been introduced as the "zigzag" model, by incorporating pathogen-secreted effector molecules that suppress host immune responses, but that may subsequently be recognized by newly evolved immune receptors, in turn (Jones and Dangl 2006). In this model, the first line of defense is governed by plasma membrane-localized pattern recognition receptors (PRRs) that detect conserved microbe-associated molecular patterns (MAMPs) to activate MAMP-triggered immunity (MTI). In subsequent layers of defense effectors are recognized by corresponding resistance proteins (R proteins), resulting in effector-triggered immunity (ETI). Although initially portrayed as separate layers of defense, numerous studies on various plant-microbe interactions have revealed that the delineation between MTI and ETI is not strict, but rather a continuum (Thomma et al. 2011). Moreover, the conceptual conflict that MAMPs are defined from the perspective of the host whereas effectors are defined from the perspective of the invader creates a conceptual conflict and has recently inspired the formulation of the Invasion Model, in which host receptors (termed invasion pattern receptors; IPRs) detect either an externally encoded or modified-self ligand that betrays invasion (termed invasion patterns; IPs) (Cook et al. 2015). In this model, any molecule can serve as an IP that is detected by an IPR, but the probability of a particular ligand-receptor complex to evolve within the framework of host immunity increases with increasing ligand probability to retain function, conservation across organisms, importance in establishment of symbiosis, and accessibility (Cook et al. 2015).

Verticillium dahliae is a xylem invading fungal pathogen that causes Verticillium wilt diseases in a wide range of plant species worldwide (Fradin and Thomma 2006). $V$. dahliae persists in the soil and enters plants through their roots. Once inside the root, the fungus grows intercellularly and invades the xylem vessels, where it sporulates to spread through the vascular system. Typical symptoms of $V$. dahliae infection include stunting, wilting, chlorosis, and necrosis (Fradin and Thomma 2006). In tomato, a single dominant locus that confers Verticillium resistance has 
been identified that controls isolates that are assigned to race 1 (Schaible et al. 1951). The locus comprises two genes, Ve1 and Ve2, that both encode extracellular leucine rich repeat (eLRR) receptor-like proteins (RLPs), and of which only Ve1 acts as a functional Verticillium resistance gene in tomato (Fradin et al. 2011; Fradin et al. 2009; Kawchuk et al. 2001; Wang et al. 2010). Functional homologs of Ve1 were reported from Nicotina glutinosa and from cotton (Chen et al. 2016; Yang et al. 2014; Zhang et al. 2012; Zhang et al. 2011; Zhang et al. 2013).

Through comparative population genomics, the $V$. dahliae effector protein that is recognized by the Ve1 immune receptor of tomato was identified as Ave1 (for Avirulence on Ve1 tomato 1) (de Jonge et al. 2012; Fradin et al. 2009). Although the intrinsic function of $V$. dahliae Ave1 (VdAve1) remains elusive, it is clear that Ave1 contributes to fungal virulence on susceptible plant genotypes (de Jonge et al. 2012). Interestingly, Ave1 homologs were identified from a number of fungal pathogens, including the tomato pathogen Fusarium oxysporum f. sp. lycopersici (FoAve1), the sugar beet pathogen Cercospora beticola (CbAve1) and the Brassicaceae pathogen Colletotrichum higginsianum (ChAve1). Strikingly, however, most Ave1 homologs were found in plants, with the most closely related homologs derived from tomato (Solanum lycopersicum; SIPNP) and grape (Vitis vinifera; VvAve1). Finally, a more distantly related homolog was identified in the plant pathogenic bacterium Xanthomonas axonopodis pv. citri (XacPNP) (de Jonge et al. 2012; Nembaware et al. 2004). Co-expression of SIPNP, FoAve1, and CbAve1 with tomato Ve1 in tobacco triggers a hypersensitive response (HR), whereas co-expression of ChAve1 with tomato Ve1 did not lead to an HR (de Jonge et al. 2012). Consequently, Ve1 was found to mediate resistance towards F. oxysporum in tomato, demonstrating involvement of this tomato immune receptor in resistance against multiple fungal pathogens (de Jonge et al. 2012).

It has previously been demonstrated that eLRR-containing cell-surface immune receptors recognize peptide sequences as epitopes of their pathogen ligands. For example, flg22 is the 22 amino acid peptide derived from bacterial flagellin that is perceived by the RLK-type immune receptor FLS2 (Zipfel et al. 2004), while the Arabidopsis EFR RLK-type immune receptor was shown to recognize elf18, an 18 amino acid peptide derived from bacterial EF-Tu (Zipfel et al. 2006). Similarly, a surface-exposed pentapeptide TKLGE of the $22 \mathrm{kDa}$ ethylene-inducing xylanase (EIX) from the biocontrol fungus Trichoderma viride determines recognition by the tomato RLP-type receptor LeEIX2 (Ron and Avni 2004; Rotblat et al. 2002). Furthermore, the tyrosine-sulfated 21 amino acid sequence RaxX21-sY present in Xanthomonas oryzae pv. oryzae RaxX is sufficient to activate XA21-mediated immunity (Pruitt et al. 2015). Finally, a conserved 20-24 amino acid sequence derived from Nep1-like proteins (NLPs) is sufficient to activate RLP23-mediated immunity in 
Arabidopsis (Albert et al. 2015; Böhm et al. 2014; Oome et al. 2014). In the present study, we aimed to identify a minimal motif in VdAve1 that determines recognition by tomato immune receptor Ve1. Our approach was based on alignment of differentially recognized Ave1 homologs, followed by a combination of deletions, domain swaps among Ave1 homologs and mutagenesis.

\section{Results}

\section{Conservation among Ave 1 homologs}

Previously, we reported the cloning of Ave 1 from $V$. dahliae and described the absence of allelic variation among 85 Ave 1 alleles of Verticillium strains (de Jonge et al. 2012). The Ave1 alleles were derived from V. dahliae and V. albo-atrum (presently V. alfalfae; (Inderbitzin et al. 2011)), whereas Ave1 alleles were not identified in any of the race 2 strains analysed, nor in $V$. dahliae and $V$. albo-atrum strains that are not pathogenic on tomato, nor in $V$. longisporum or $V$. tricorpus (de Jonge et al. 2012). To further explore Ave1 diversity, we assessed its presence in a collection of 97 Verticillium strains isolated from various host plants and different geographical locations, resulting in the identification of 17 novel Ave 1 alleles (Supplemental Table 1). No allelic variation was found among the newly identified Ave1 alleles amplified from $V$. dahliae as well as from $V$. alfalfae and $V$. nonalfalfae, two novel species that have recently been separated from V. albo-atrum (Inderbitzin et al. 2011). However, an Ave 1 homolog was identified in four isolates of $V$. nubilum (VnAve1), a species that is mainly known as a saprophytic and opportunistic pathogen (Isaac 1953). While the predicted VnAve1 protein sequence displays 13 amino acid polymorphisms when compared with VdAve1 (Figure 1A), the four VnAve1 alleles were found to be identical to each other.

Alignment of the amino acid sequences of VdAve1 with homologs from plants (SIAve1 and VvAve1), and plant pathogens (VnAve1, FoAve1, CbAve1, ChAve1 and XacPNP) reveals blocks of highly conserved amino acids that are alternated with more variable regions (Figure 1A). Based on prediction by SignalP 4.0 (Petersen et al. 2011), all Ave1 homologs contain $\mathrm{N}$-terminal signal peptides that direct secretion of the molecules into the extracellular space (Figure 1A; D-cutoff score $>0.6$ ). Moreover, the four cysteine residues that are present in VdAve1 are conserved among all homologous proteins (Figure 1A), and in silico analysis using DISULFIND (Ceroni et al. 2006) suggests the formation of disulphide bridges between Cys35 and Cys63, as well as between Cys71 and Cys79. From the alignment it is apparent that XacPNP is the most divergent, while all other homologs are relatively comparable (Figure 1A). 


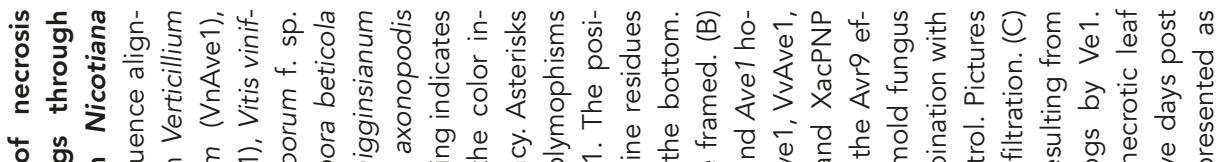

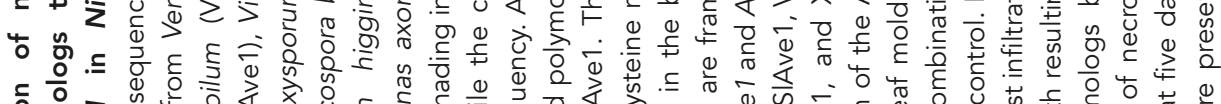

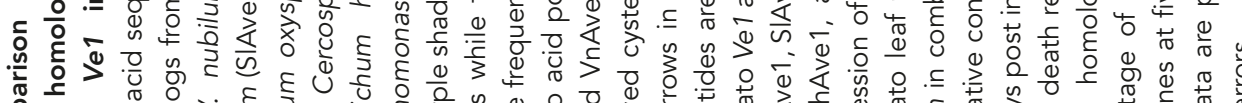

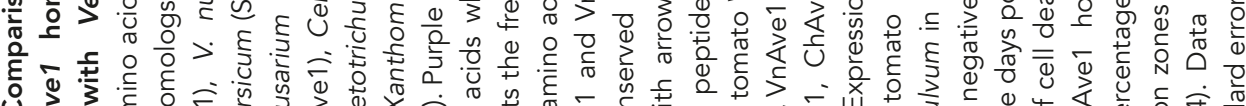

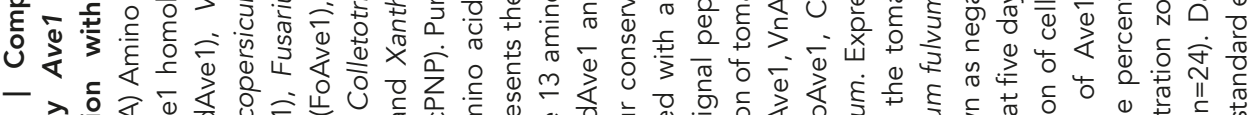

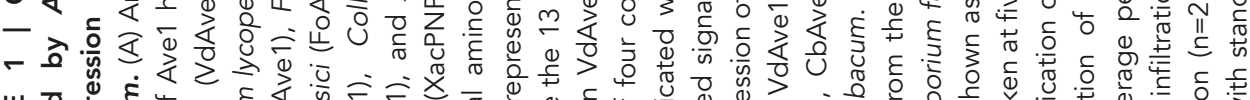

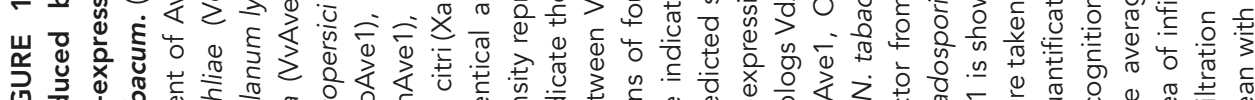

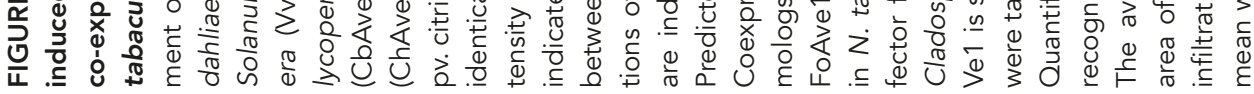

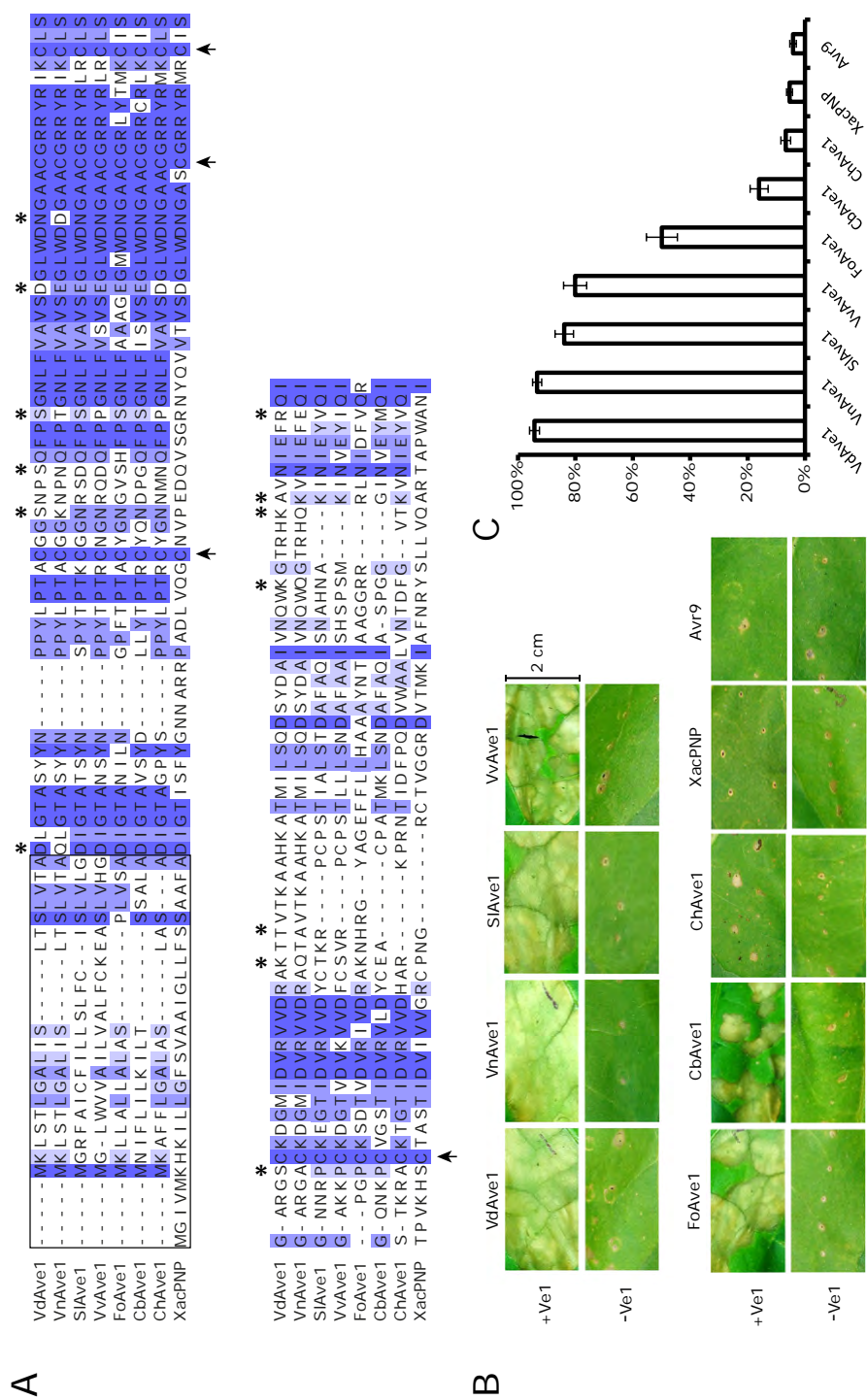




\section{Comparison of necrosis-inducing activity of Ave 1 homologs}

It was previously demonstrated that Ve1 recognizes not only VdAve1, but also SIPNP, FoAve1 and CbAve1 (de Jonge et al. 2012). We now also tested the HR-inducing capacity of $V_{n} A v e 1, V_{v} A v e 1$ and XacPNP that were isolated from $V$. nubilum, $V$. vinifera and $X$. axonopodis, respectively. Co-expression of the sequence-unrelated effector Avr9 from the tomato leaf mould fungus Cladosporium fulvum (van Kan et al. 1991) in combination with Ve1 served as a negative control. Whereas expression of VnAve1 or VvAve1 together with Ve1 in Nicotiana tabacum resulted in strong $H R$, co-expression of XacPNP or Avr9 with Ve1 triggered little to no necrosis in addition to the small wounds that were caused by the infiltration procedure (Figure 1B). To compare the HR induced by the various Ave1 homologs, they were coexpressed with Ve1 in N. tabacum and HR development was measured at five days post infiltration by quantification of the leaf area that developed necrosis (Zhang et al. 2013). Importantly, none of the Ave1 homologs induced necrosis in absence of Ve1 (Figure 1B). Whereas agroinfiltration of either VdAve1 or VnAve1 with Ve1 resulted in complete necrosis of the infiltrated leaf area, agroinfiltration of FoAve1 with Ve1 resulted in large necrotic spots in the infiltrated leaf area, although no complete collapse of the infiltrated area was observed. Upon agroinfiltration of CbAve1 with Ve1, spreading of smaller and larger necrotic spots was observed in all infiltrated areas, but the infiltrated leaf area did not turn completely necrotic. For ChAve1, XacPNP and Avr9, no spreading necrosis was observed beyond the wounded infiltration sites (Figure 1B). Upon agroinfiltration of the tomato and grape homologs, SIAve1and VvAve1, most of the infiltrated leaf area developed necrosis, occasionally affecting the complete infiltrated leaf sector. To confirm that reduced levels of HR are not due to instability of the proteins in the expression assay, GFP-tagged Ave1 homologs were detected by immunoblotting. Similar to GFP-tagged VdAve1 protein or GFP-tagged VnAve1 that induce the strongest HR, all other GFP-tagged Ave1 homologs accumulated to clearly detectable protein levels (Supplemental Figure 1).

\section{The C-terminus of VdAve 1 is required for recognition by $\mathrm{Ve} 1$}

In order to permit functional analyses, a construct encoding C-terminal protein GFP fusion of VdAve1 was generated. However, C-terminal fusion of a GFP tag to Ave1 resulted in loss of recognition by Ve1. Considering that the GFP tag is relatively large, we engineered C-terminal fusions to VdAve1 with smaller protein tags. Nevertheless, all C-terminal tags abolished, or significantly reduced, HR development (Figure 2A; C). Importantly, the C-terminal GFP fusions could be detected by immunoblotting (Supplemental Figure 2), suggesting that accessibility of the VdAve1 C-terminus is important for recognition by Ve1. 
A

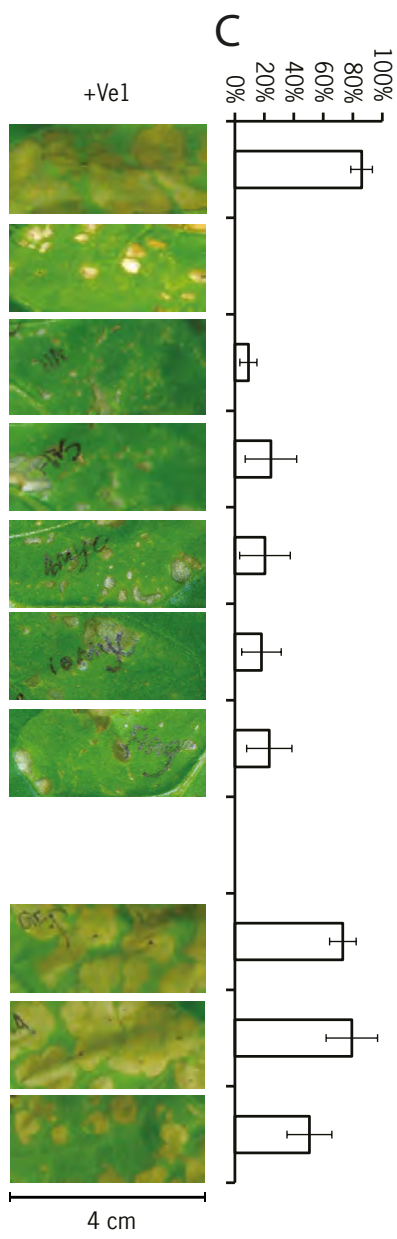

B
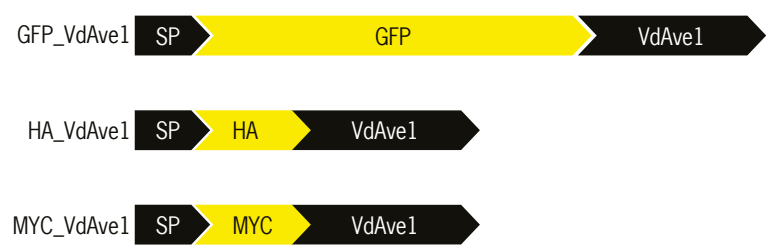

VdAve1_GFP

$\mathrm{SP}>\mathrm{VdAve1}$

GFP

VdAve1_HA

$\mathrm{SP}>$ VdAvel

$\mathrm{HA}$

$\begin{array}{lll}\text { VdAve1_HIS } & \text { SP }>\quad \text { VdAvel }\end{array}$

HIS

VdAve1_4MYC $S P>$ VdAve1 $>4 \times M Y C$

VdAve1_10MYC $S P>$ VdAve1 $>10 x M Y C$

VdAve1_FLAG $S P>$ VdAve1 $>$ FLAG

GFP_VdAve1 SP $>$ GFP
HA_VdAve1 $\mathrm{SP}>\mathrm{HA}>$ VdAvel
MYC_VdAve1 $\mathrm{SP}>\mathrm{MYC}>$ VdAvel

FIGURE 2 | C-terminal fusion of a GFP tag to VdAve1 results in loss of recognition by Ve1. (A) The signal peptide of VdAve1 (SP) directs secretion of untagged VdAve1 (VdAve1), C-terminally tagged VdAve1 (VdAve1_GFP, VdAve1_HA, VdAve1_HIS, VdAve1_4MYC, VdAve1_10MYC, VdAve1_FLAG) into the extracellular space. The constructs were co-expressed with Ve1 in $N$. tabacum upon agroinfiltration and the occurrence of the hypersensitive response was monitored. (B) The signal peptide of VdAve1 (SP) directs secretion of N-terminally tagged VdAve1 (GFP_ VdAve1, HA_VdAve1 and MYC_VdAve1) into the extracellular space. The constructs were coexpressed with $V_{e} 1$ in $N$. tabacum upon agroinfiltration and the occurrence of the hypersensitive response was monitored. All pictures were taken at five days post infiltration. (C) Quantification of cell death resulting from recognition of tagged VdAve1 proteins by Ve1. The graph shows the average percentage of necrotic leaf area of infiltration zones at five days post infiltration $(n>5)$. Data are presented as mean with standard deviations.

To further investigate the role of the C-terminus in recognition of VdAve1 by Ve1, a number of $\mathrm{C}$-terminal truncations was generated. Deletion of 42 amino acids of the C-terminus, from Lys93 to lle134 (VdAve1_M1), resulted in loss of recognition by 
Ve1 (Figure 3). Subsequent analysis of step-wise smaller truncations revealed that already a C-terminal deletion of nine amino acids (VdAve1_M4) resulted in loss of Ve1 recognition (Figure 3), despite the expression of stable protein (Supplemental Figure 2).

We subsequently performed complementation experiments in V. dahliae to confirm the importance of the C-terminus for immune activation. To this end we expressed the VdAve1_M4 construct (Figure 3) in a V. dahliae VdAve1 deletion strain and inoculated Ve1 tomato with this transgenic fungus. Plants that were inoculated with $V$. dahliae expressing this VdAve1 construct showed a similar disease phenotype as plants inoculated with a VdAve1 deletion strain, whereas plants inoculated with wild type $V$. dahliae and the VdAve 1 complementation strain resembled mock-inoculated plants (Figure 4A; B). Collectively, our results show that the C-terminal nine amino acids of $\mathrm{VdAve} 1$ are required to establish Ve1-mediated immunity.

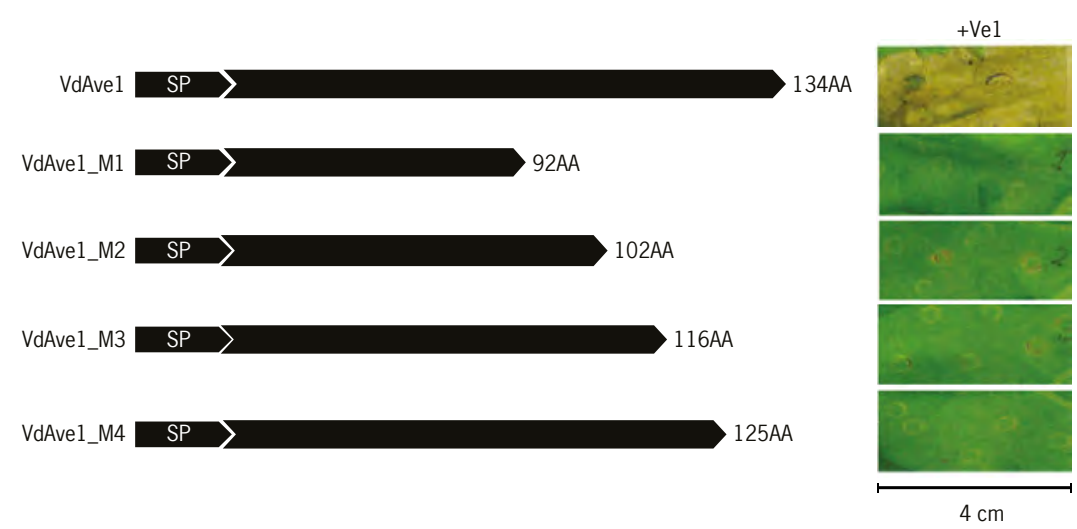

FIGURE 3 | Deletion of the C-terminal nine amino acids from VdAve1 results in loss of recognition by $\mathrm{Ve1}$. Occurrence of the hypersensitive response in $\mathrm{N}$. tabacum upon co-expression of VdAve1 truncations with Ve1. Constructs encoding full length VdAve1 with its native signal peptide (SP) and four truncations that lack the C-terminal 42 (VdAve1_M1), 32 (VdAve1_M2), 18 (VdAve1_M3) and 9 (VdAve1_M4) amino acids of VdAve1 were tested. Pictures were taken at five days post infiltration.

\section{Ave 1 recognition cannot be assigned to a single amino acid residue}

Since the C-terminal nine amino acids appear to be essential for $V d A v e 1$ recognition, and the bacterial homolog XacPNP that is significantly divergent in this region (Figure $1 \mathrm{~A}$ ) is not recognized by $\mathrm{Ve} 1$ (Figure $1 \mathrm{~B}$ and $\mathrm{C}$ ), an expression construct for a chimeric Ave1 protein was engineered in which the C-terminal nine amino acids of VdAve1 were replaced by those of XacPNP (Vd_Xac9AA; Figure 5A; D). As expected, co-expression of the $\mathrm{Vd}$ Xac9AA chimera with $\mathrm{Ve} 1$ in $\mathrm{N}$. tabacum resulted in absence of recognition, as only small necrotic spots were observed at 
most (Figure 5A). Conversely, a chimeric Xac_Vd9AA protein was constructed, in which the last nine amino acids of XacPNP were replaced by those of VdAve1. Coexpression of Xac_Vd9AA with Ve1 in $\mathrm{N}$. tabacum resulted in a relatively strong HR, although full necrosis was not observed in the infiltrated leaf area (Figure 5A; D). With immunodetection the stability of the chimeric Vd_Xac9AA and Xac_Vd9AA proteins was confirmed (Supplemental Figure 3).

A

C

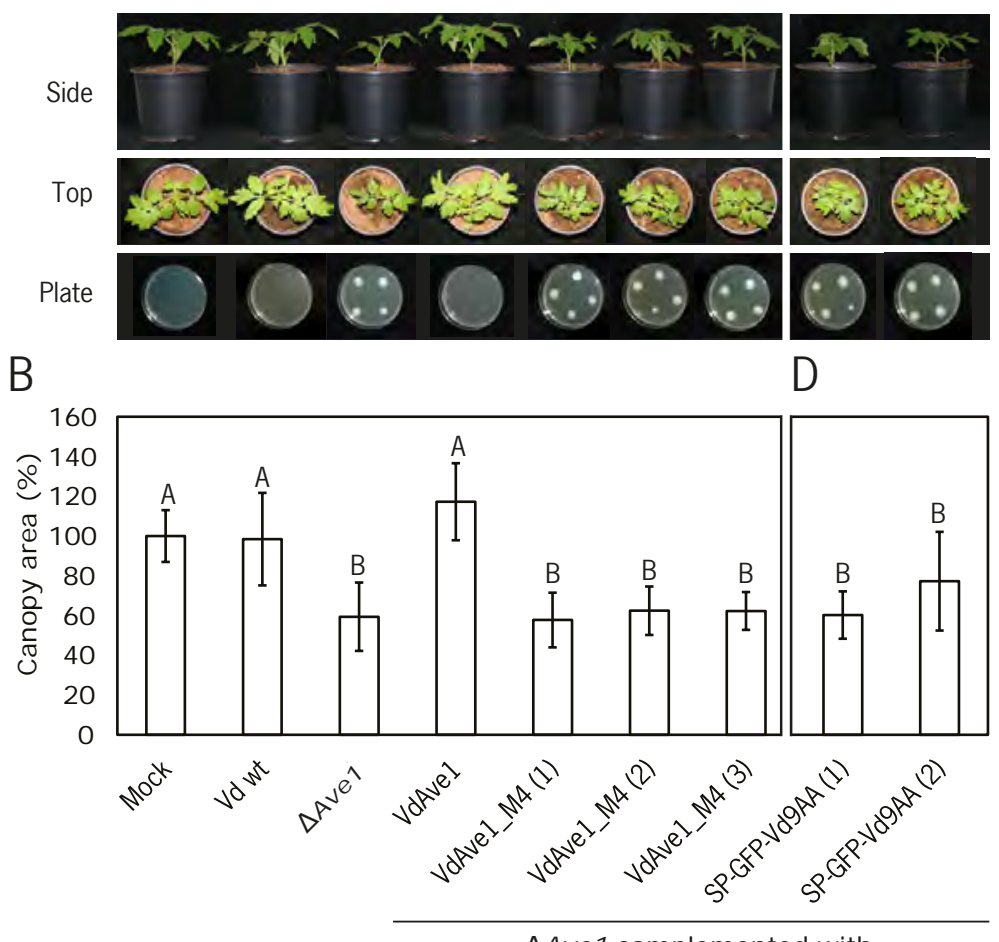

$\Delta$ Avel complemented with

FIGURE 4 | Complementation experiments in V. dahliae confirm that the C-terminal nine amino acids are required to activate Ve1 immunity in tomato. (A) V. dahliae Ave1 deletion strains expressing a construct encoding VdAve1 lacking the C-terminal 9 amino acids (VdAve1_ M4) are unable to activate $V e 1$ immunity in tomato when compared with $V$. dahliae wild-type (Vd wt) and genetic complementation (VdAve1). (B) Quantification of the tomato canopy area $(\mathrm{n}=8)$. Data are presented as mean with standard deviations. Different letters indicate significant differences $(\mathrm{P}<0.05$; One-Way ANOVA). (C) V. dahliae Ave1 deletion strains expressing a construct that encodes the C-terminal 9 amino acids fused to GFP (SP-GFP-9AA) are unable to activate Ve1 immunity in tomato compared to $V$. dahliae wild-type $(\mathrm{Vd} w \mathrm{t})$ and genetic complementation (VdAve1). (D) Quantification of the tomato canopy area $(n=8)$. Data are presented as mean with standard deviations. Different letters indicate significant differences $(P<0.05$; One-Way ANOVA). 
A

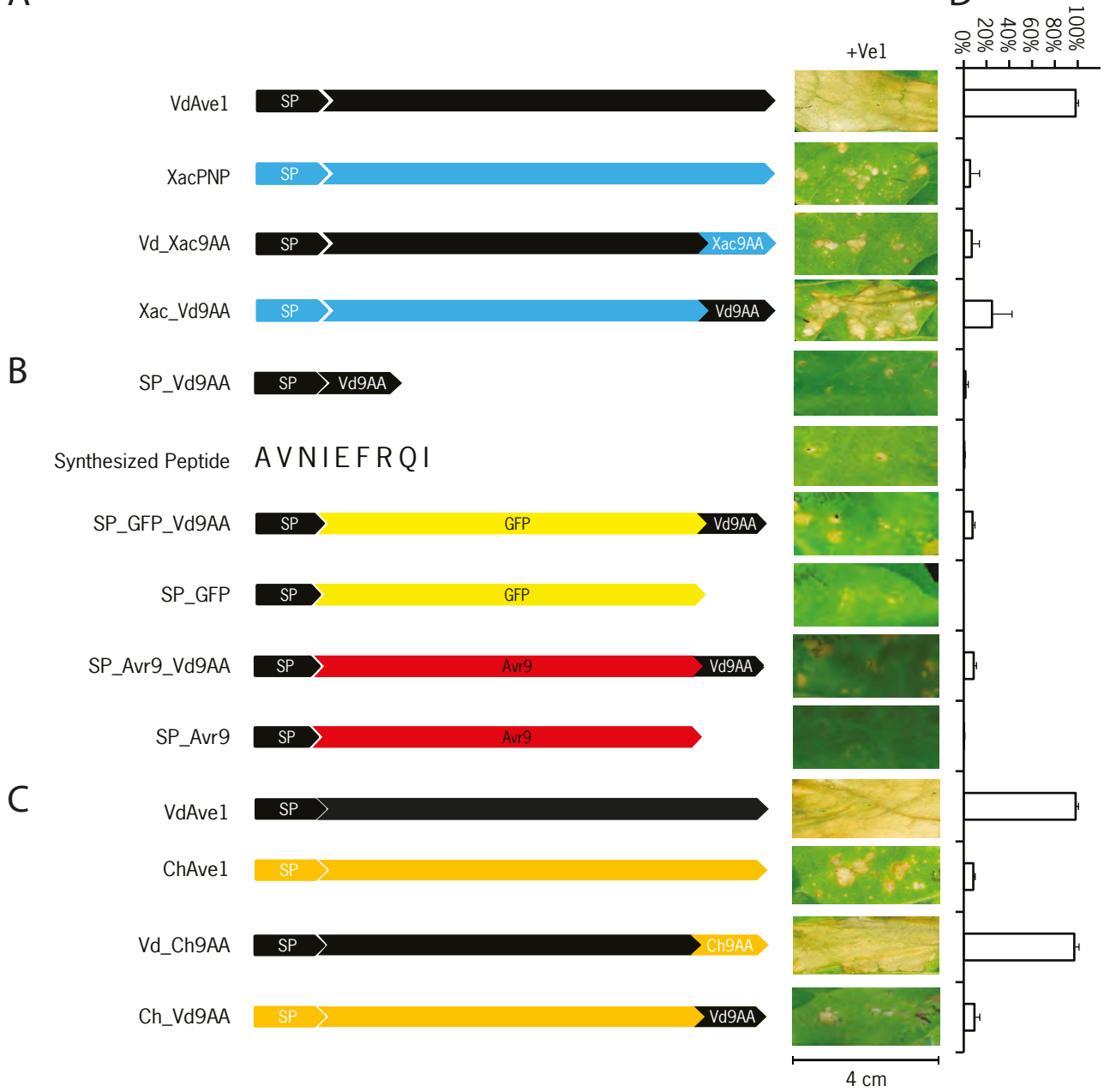

FIGURE 5 | Domain swaps reveal the importance of the C-terminal nine amino acids of Ave1 homologs for recognition by Ve1. (A) Occurrence of the hypersensitive response in N. tabacum upon co-expression of Ave1 chimeras with Ve1. Constructs encoding full length VdAve1 and XacPNP with their native signal peptides (SP) and two chimeras in which their $\mathrm{C}$-terminal nine amino acids were exchanged were tested. In chimera Vd_Xac9AA the C-terminal nine amino acids of VdAve1 (Vd9AA) were replaced with those of XacPNP (Xac9AA), while in chimera Xac_Vd9AA Xac9AA was replaced by Vd9AA. (B) Construct SP_9AA encodes the C-terminal nine amino acids of $\mathrm{VdAve1}$ (Vd9AA) fused to the $\mathrm{VdAve} 1$ signal peptide (SP). SP_GFP_Vd9AA encodes GFP that is C-terminally tagged with Vd9AA, and N-terminally fused to the VdAve1 signal peptide (SP) to establish extracellular targeting, while SP_GFP lacks the C-terminal Vd9AA fusion. Furthermore, a chemically synthetized peptide encompassing Vd9AA (AVNIEFROI) was used. (C) Constructs encoding full length VdAve1 and ChAve1 with their native signal peptides (SP) and two chimeras in which their C-terminal 9 amino acids were exchanged were tested. In chimera Vd_Ch9AA the C-terminal nine amino acids of VdAve1 (Vd9AA) were replaced with those of ChAve1 (Ch9AA), while in chimera Ch_Vd9AA Ch9AA was replaced byVd9AA. All pictures were taken at five days post infiltration. (D) Quantification of cell death resulting from recognition of chimera proteins by Ve1. The average percentage of necrotic leaf area of infiltration zones at five days post infiltration $(n>10)$. Data are presented as mean with standard deviation. 
To further unravel how the C-terminal nine amino acids contribute to recognition of VdAve1, we performed site-directed mutagenesis. Since the first of the nine residues (Ala126) is shared with XacPNP, we conclude that this one likely does not contribute to $\mathrm{VdAve} 1$ recognition. Thus, eight alanine scanning constructs were engineered in which the remaining residues were substituted by alanine (V127A, N128A, I129A, E130A, F131A, R132A, Q133A, and I134A, respectively; Figure 6A; B). The mutant constructs were tested in N. tabacum upon co-expression with Ve1, and $\mathrm{HR}$ development was measured at five days post infiltration by quantification of the leaf area that developed necrosis (Figure 6C). Similar to VdAve1, mutants R132A and Q133A activated strong Ve1-mediated HR, as $>90 \%$ of the infiltrated leaf area developed necrosis (Figure 6C). In contrast, mutants N128A, I129A, E130A and $\mathrm{F} 131 \mathrm{~A}$ triggered clearly reduced necrosis, with $<70 \%$ of the infiltrated leaf area developing necrosis (Figure 6C). Alanine substitution of Val127 and Ile134 (V127A and I134A, respectively) resulted in an intermediate reduction of necrosis-inducing activity, with $70-80 \%$ of the infiltrated leaf area developing necrosis (Figure 6C).

To further investigate the contribution of individual amino acids to recognition by $V e 1$, we engineered five additional mutants in which individual residues of the VdAve1 C-terminal nine amino acids were substituted by corresponding residues from XacPNP (V127R, N128T, E130P, F131W, and Q133N; Figure 6A; B). Whereas expression of $\mathrm{F} 131 \mathrm{~W}$ and $\mathrm{Q} 133 \mathrm{~N}$ induced similar necrosis as wild type VdAve1, mutants N128T and E130P exhibited significantly compromised HR upon coexpression with $V e 1$, with $<60 \%$ of the infiltrated leaf area developing necrosis (Figure 6C). Mutant V127R did not induce HR at all in the presence of Ve1 (Figure 6C). Importantly, all site-directed mutants that showed compromised HR-inducing capacity accumulated to similar levels as VdAve1 or XacPNP (Supplemental Figure 4). These observations suggest that the region from Val127 to Phe131 is critical for recognition by Ve1. Collectively, we conclude that the C-terminal nine amino acids of $\mathrm{VdAve} 1$ are required for recognition by $\mathrm{Ve} 1$, and that recognition cannot be assigned to a single amino acid residue. 
VdAvel $\mathrm{ANIEFRI}$ VnAvel KVNIEFEQI SIAvel KINIEYVQI VuAve1 KI NVEYIQI FoAve1 RINIDFVQR CbAvel GINVEYMQI ChAve1 KVNIEYVQI XacPNP A RT APWAN I

\begin{tabular}{|c|c|c|c|}
\hline VdAve 1 & $\mathrm{SP}$ & VdAve1 $\triangle 9 A A$ & $\triangle A \vee N I E F R$ \\
\hline $127 \mathrm{~A}$ & $S P$ & VdAve1 $\triangle 9 A A$ & $\triangle A A N I E F R Q$ \\
\hline N128A & SP & VdAve1 $\triangle 9 A A$ & $\triangle A \vee A \mid E F R Q$ \\
\hline $1129 \mathrm{~A}$ & SP & $\overline{V d A v e 1} \triangle 9 A A$ & AVNAEFRQ \\
\hline E130A & $\mathrm{SP}$ & VdAvel $\triangle 9 A A$ & $\triangle A V N \mid A F R Q$ \\
\hline $\mathrm{F} 131 \mathrm{~A}$ & SP & VdAve1 $\triangle 9 A A$ & $\triangle A V N I E A R Q$ \\
\hline R132A & SP & VdAve1 $\triangle 9 A A$ & S V NIEFAQ \\
\hline & SP & VdAvel $\triangle 9 A A$ & $\triangle A V N I E F R A$ \\
\hline & $S P$ & VdAvel $\triangle 9 A A$ & $\triangle A V N I E F R Q A$ \\
\hline V1 & $S P$ & VdAve1 $\triangle 9 A A$ & ARNIEFR \\
\hline N128T & $S P$ & VdAve1 $\triangle 9 A A$ & $\triangle A \vee T I E F R$ \\
\hline E130P & $S P$ & VdAve1 $\triangle 9 A A$ & $\triangle A \vee N I P F R Q$ \\
\hline F131W & $S P$ & $\begin{array}{l}\text { VdAve1 } \triangle 9 A A \\
\end{array}$ & $\triangle A \vee N I E W R Q$ \\
\hline & $S P$ & VdAvel $\triangle 9 A A$ & $>$ A VNIEFR N \\
\hline
\end{tabular}

C

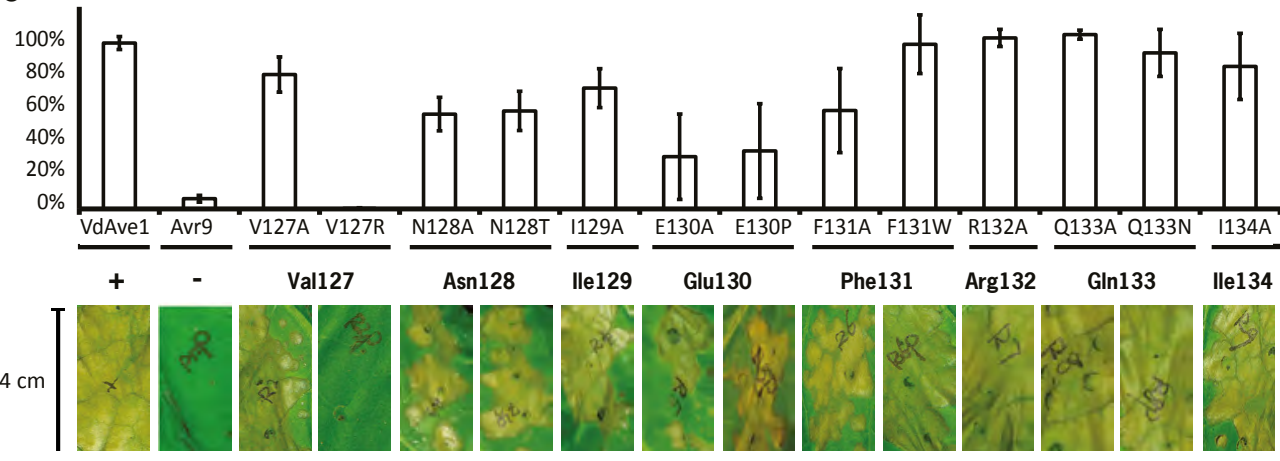

FIGURE 6 | Contribution of individual amino acid residues to recognition of the C-terminal nine amino acids of VdAve1. (A) Amino acid sequence alignment of the C-terminal nine amino acids of various Ave1 homologs. Identical and highly similar residues are indicated with black shading whereas weakly similar residues are indicated with grey shading. (B) Schematic representation of mutant proteins V127A, N128A, I129A, E130A, F131A, R132A, Q133A, I134A, V127R, N128T, E130P, F131W, and Q133N, respectively. Bold characters indicate the mutated residues. (C) Coexpression of tomato Ve1 and VdAve1 mutants in N. tabacum. The quantification of cell death resulting from recognition of VdAve1 mutants by $\mathrm{Ve} 1$ is shown as the average percentage of necrotic leaf area of infiltration zones at five days post infiltration $(n>5)$. Data are presented as mean with standard errors.

\section{The C-terminal nine amino acids of VdAve 1 are not sufficient for recognition by $\mathrm{Ve} 1$}

To determine whether the $\mathrm{C}$-terminal nine amino acids are sufficient to trigger Ve1mediated recognition, we generated a construct encoding the C-terminal nine amino acids of VdAve1 fused to the VdAve1 signal peptide (SP_Vd9AA) (Figure $5 B$; D). This construct was co-expressed with $V e 1$ in N. tabacum, but no necrosis was observed in the infiltrated leaf (Figure 5B). Furthermore, also infiltration of a chemically synthetized peptide encompassing the C-terminal nine amino acids of VdAve1 was not able to trigger an HR in Ve1-expressing tobacco up to a 
concentration of $1 \mathrm{mg} / \mathrm{mL}$ (Figure 5B; D). We speculated that the nine amino acid peptide may not be stable upon secretion in the apoplast, irrespective whether it is in planta expressed or injected upon chemical synthesis. In an attempt to overcome such complication, we generated constructs in which the coding sequence of GFP or Avr9 was $\mathrm{N}$-terminally fused to the $\mathrm{VdAve} 1$ signal peptide and C-terminally fused to the C-terminal nine amino acids of VdAve1 (SP_GFP_Vd9AA and SP-Avr9_Vd9AA; Figure $5 B ; D)$. As negative controls, we generated constructs in which the coding sequence of GFP or Avr9 was N-terminally fused to the VdAve1 signal peptide without the C-terminal nine amino acids of VdAve1 (SP_GFP and SP_Avr9; Figure 5B; D). All constructs were co-expressed with Ve1 in N. tabacum and HR development was monitored at five days post infiltration. However, only slight necrosis was found in the infiltrated sector despite the observation that the chimeric GFP proteins could be detected upon immunoblotting (Supplemental Figure 2).

Subsequently, a complementation assay was performed in $\mathrm{V}$. dahliae to confirm that the $\mathrm{C}$-terminal nine amino acids are not sufficient to activate immunity. To this end we expressed the SP_GFP_Vd9AA construct in the Ave1 deletion strain of V. dahliae (Figure 4C; D) and inoculated Ve1 tomato. Plants inoculated with strains expressing SP_GFP_Vd9AA showed similar symptoms as plants inoculated with the VdAve1 deletion strain, whereas plants inoculated with wild type $V$. dahliae and the VdAve1 complementation strain displayed similar symptoms as mock-inoculated plants (Figure 4A; D). Collectively, our results show that the C-terminal nine amino acids of $\mathrm{VdAve} 1$ are required but not sufficient to establish Ve1-mediated immunity.

\section{Evidence for contribution to recognition in addition to the C-terminal nine amino acids}

ChAve1 is closely related to VdAve1 as six of the nine C-terminal amino acids are identical. Nevertheless, only weak HR is detected upon co-expression of ChAve1 and Ve1 in N. tabacum (Figure 1B; C). To further investigate recognition of the $\mathrm{C}$-terminal nine amino acids of ChAve1, an expression construct encoding a chimeric Ave1 protein was engineered in which the C-terminal nine amino acids of $\mathrm{VdAve} 1$ were replaced by those of ChAve1 (Vd_Ch9AA). Intriguingly, expression of $\mathrm{Vd}_{-}$ Ch9AA resulted in complete necrosis of the infiltrated leaf area upon co-expression with Ve1 in N. tabacum, whereas the reciprocal swap in which the C-terminal nine amino acids of ChAve1 were replaced by those of VdAve1 (Ch_Vd9AA) was not recognized by Ve1 (Figure $5 C$; D). With immunodetection the stability of the chimeric Vd_Ch9AA and Ch_Vd9AA proteins was confirmed (Supplemental Figure 5). These data provide further support for the finding that the C-terminal nine amino acids of VdAve1 are not sufficient to establish Ve1-mediated recognition, and confirm that additional requirements for the occurrence of the HR reside outside the stretch of the C-terminal nine amino acids. 


\section{A structural model of Avel suggests a contribution of both the $\mathrm{N}$ - and \\ C-terminus of Ave 1 to Vel recognition}

In an attempt to get a better insight in Ave1 recognition by Ve1 we generated a structural model of Ave1. Structural comparison with the protein databank (RCSB PDB) (Rose et al. 2013) revealed the maize protein EXPB1 (PDB ID: 2HCZ) (Yennawar et al. 2006) as a potential structural analogue (TM-Score 0.84) of VdAve1. The Ave1 structural model shows that the C-terminal nine amino acids (shown in orange in Figure 7) are exposed at the surface of the Ave1 protein. The model also predicts that the $\mathrm{N}$-terminus (shown in blue in Figure 7) congregates with the C-terminus, which led us to hypothesize that Ve1 potentially recognizes a patch of the Ave1 protein that includes both the $\mathrm{N}$ - and $\mathrm{C}$-terminal. To test this hypothesis, we fused various tags to the $\mathrm{N}$-terminal of Ave1, between the signal peptide and the mature peptide. Surprisingly, while N-terminal tagging of Ave1 with GFP and HA did not affect recognition, $\mathrm{N}$-terminal tagging with $\mathrm{MYC}$ compromised recognition (Figure $2 \mathrm{~B}$; C). Immuno blotting confirmed that the GFP fusions were stably expressed (Supplemental Figure 2).
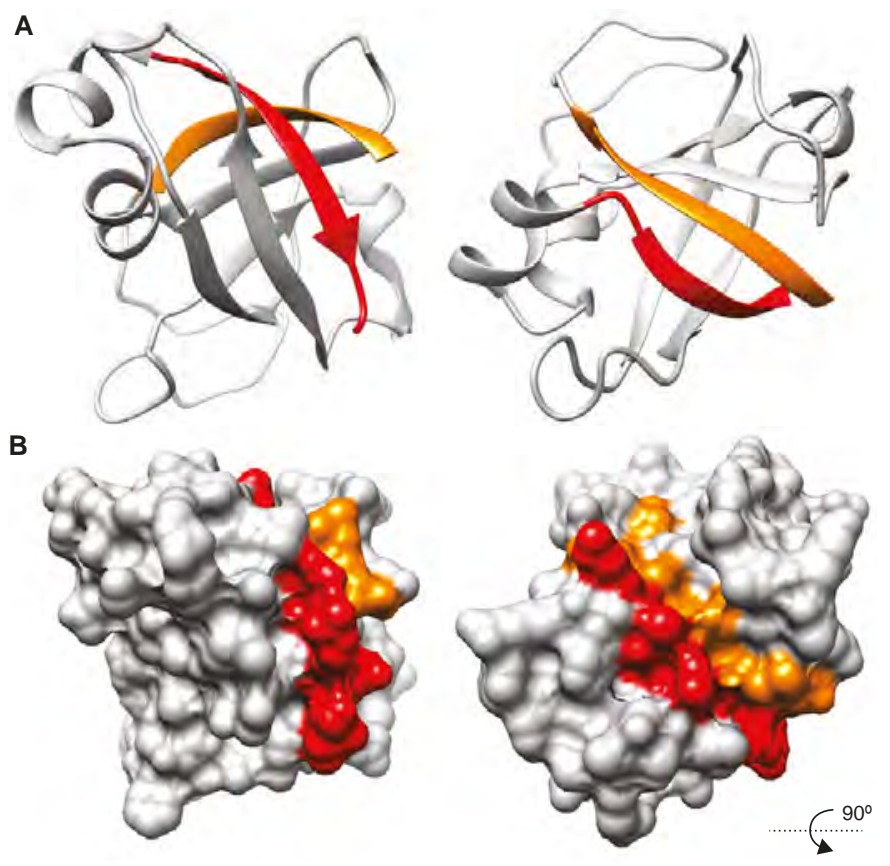

FIGURE 7 | Model for the 3D structure of the Verticillium dahliae effector VdAve1. The V. dahliae VdAve1 structure was predicted using I-TASSER (Zhang 2008). The inferred VdAve1 structure is of high quality indicated by a confidence score (C-Score) of 1.22, and displayed as a ribbon (A), and surface (B) model from the side (left) and the top (right). The C-terminal nine amino acid sequence of $\mathrm{VdAve} 1$ is indicated in orange, while the $\mathrm{N}$-terminal eight amino acid sequence is indicated in blue. 


\section{Discussion}

\section{The C-terminal nine amino acids of Avel are required for Ve1-mediated recognition}

We have previously shown that the $V d A v e 1$ effector of $V$. dahliae is recognized by the tomato immune receptor $V e 1$, and we identified several homologs in other species that are differentially recognized by Ve1 (Figure 1B; C) (de Jonge et al. 2012). In this study, we demonstrate that the C-terminal nine amino acids of $\mathrm{VdAve} 1$ are required for the recognition by $\mathrm{Ve} 1$, as truncations of $\mathrm{VdAve} 1$ lose recognition. The sequence of the C-terminal nine amino acids of the Ave1 homologs that are recognized (VdAve1, VnAve1, SIPNP, VvAve1, FoAve1, CbAve1 and also ChAve1) only share Asn128 and Gln133 that are completely conserved (Figure 6A). However, the other amino acids in this region are characterized by a high degree of conservation. A similar sequence variation in peptide epitopes, allowing substitutions by amino acids with similar chemical properties has previously been observed, for instance in flagellin, CLV3 and AtPep1 (Kondo et al. 2008; Naito et al. 2008; Pearce et al. 2008; Song et al. 2012).

To further analyse the contribution of individual amino acids in the C-terminal nine amino acids to recognition by Ve1, we performed site-directed mutagenesis on VdAve1. The sequence alignment and mutagenesis analysis suggest that the five neighbouring residues Val127, Asn128, Ile129, Glu130 and Phe131 are the major determinants of recognition of the C-terminal nine amino acids. Whereas alanine substitution of Val127 (V127A) only slightly reduced the HR-inducing capacity of VdAve1, VdAve1 cannot be recognized when Val127 is substituted by an arginine (V127R) that occurs in XacPNP on that position. Conversely, the HR-inducing capacity of VdAve1 is significantly impaired when Phe131 was replaced by the nonsimilar amino acids alanine (F131A), while substitution by tryptophan (F131W), an amino acid with similar chemical properties that occurs in XacPNP on that position, still triggers strong HR.

\section{The C-terminal nine amino acids are not sufficient to trigger Ve1-mediated recognition}

To test whether the C-terminal nine amino acids are sufficient to trigger Ve1mediated HR a synthesized peptide and a construct in which the C-terminal nine amino acids of Ave1 were fused to GFP were co-expressed with Ve1. The C-terminal nine amino acids are not sufficient to trigger Ve1-mediated recognition as neither construct encoding the C-terminal nine amino acids peptide alone nor a GFP fusion construct can activate Ve1-mediated HR. With a V. dahliae complementation assay 
we confirmed that the C-terminal nine amino acids of Ave1 are not sufficient to stop the fungus from colonizing Ve1 tomato. Altogether, these results show that the C-terminal nine amino acid sequence is required, but not sufficient, to trigger Ve1mediated immunity. Thus, recognition may involve additional residues besides the nine amino acid sequence to initiate Ve1-mediated immunity. To test if recognition involves residues immediately adjacent to the nine amino acids, GFP fusion constructs should be generated with longer C-terminal sequences.

We noticed that expression of ChAve1 with Ve1 does not trigger the activation of $\mathrm{HR}$ although an Ave1 chimera containing the C-terminal nine amino acids of ChAve1 have the potential to be recognized. Conversely, a ChAve1 chimera containing the C-terminal nine amino acids of $\mathrm{VdAve} 1$ does not trigger the activation of an HR. This also supports the hypothesis that a stretch outside the nine amino acid sequence is required to activate Ve1 immunity. However, obviously it needs to be realized that Ve1 evolved in tomato to recognize attempted host colonization by $V$. dahliae, while C. higginsianum is not a tomato pathogen and thus Ve1 did not have the chance to co-evolve with ChAve1.

\section{A structural model of Ave 1}

Plasma membrane-localized PRRs are often activated upon recognition of a short peptide sequence on the surface of their PAMP ligands, such as flg22 or flgll-28 derived from flagellin, elf18 or EFa50 derived from EF-Tu, and pentapeptide TKLGE derived from EIX (Cai et al. 2011; Furukawa et al. 2014; Rotblat et al. 2002; Zipfel et al. 2006; Zipfel et al. 2004). Similarly, a 20-24 peptide in NLP effectors conserved in organisms from three kingdoms of life was shown to be sufficient to activate the PRR receptor RLP23 in Arabidopsis (Albert et al. 2015; Böhm et al. 2014; Oome et al. 2014). However, for the flax-rust effectors AvrM and AvrL567 it has been suggested that multiple contact points are required for recognition by the corresponding flax R-proteins (Ve et al. 2013; Wang et al. 2007). Similarly, multiple residues at separate locations on the surface of the ATR1 effector of Hyaloperonospora arabidopsidis are required for recognition by the R-protein RPP1 in Arabidopsis (Chou et al. 2011; Goritschnig et al. 2016). Fusion of various tags to the C-terminus of Ave1 resulted in loss of recognition and fusion of a MYC tag to the N-terminus of Ave1 resulted in reduced Ve1-mediated $\mathrm{HR}$. We generated a structural model of Ave1 to get a better insight in the recognition by Ve1. This model shows that the $\mathrm{N}$ - and C-terminal congregate and are exposed at the protein surface. We therefore hypothesize that residues at both the $\mathrm{N}$ - and $\mathrm{C}$-terminus of Ave1 are required for recognition by Ve1. This can be tested by generating $\mathrm{N}$-terminal deletions of $\mathrm{VdAve} 1$ for co-expression with Ve1. 


\section{Involvement of C-terminal nine amino acids in Ave 1 virulence}

Ave1 has been characterized as an ortholog of plant natriuretic peptides (PNPs) (de Jonge et al. 2012). Natriuretic peptides were originally identified in animals as atrial natriuretic peptides (ANPs); polypeptide hormones that are secreted by heart muscle cells that are involved in the homeostatic control of salt and water balance and blood pressure (Potter et al. 2009). It has been demonstrated that $X$. axonopodis pv. citri utilizes a PNP homolog, XacPNP, to modulate the regulation of host plant homeostasis to establish infection (Gottig et al. 2008). The high homology between tomato SIAve1and $V$. dahliae VdAve1, likely due to acquisition of VdAve1 by $V$. dahliae from plants through horizontal gene transfer (HGT), in combination with the fact that VdAve1 acts as a virulence factor of Verticillium in susceptible tomato plants, suggests that $V$. dahliae utilizes VdAve1 to modulate host plant responses to generate conditions that favour colonization.

Absence of allelic variation in Ave1 alleles from $V$. dahliae and $V$. albo-atrum suggests that identical alleles are required for maximum virulence (de Jonge et al. 2012). However, the importance of the C-terminal amino acids for the function of VdAve1 related to virulence function is unknown. Mutational analysis within Pep13, a surface-exposed fragment of a calcium-dependent cell wall transglutaminase (TGase) from the oomycete plant pathogen Phytophthora sojae that activates plant immune responses, identified the same amino acids indispensable for both TGase activity and immune activation (Brunner et al. 2002). Similarly, amino acid residues that are required for immunity-inducing activity of the flagellin-derived peptide flg22 were found to be important for the intrinsic flagellar function, as mutant flagella were unstable and impaired in flagellar motility (Naito et al. 2008). However, in contrast, the surface exposed pentapeptide TKLGE epitope of the Trichoderma viride ethylene-inducing xylanase (EIX) that is essential for recognition by the eLRR receptor LeEIX2 is not involved in xylanase enzymatic activity (Ron and Avni 2004; Rotblat et al. 2002). Further research, involving the complementation of VdAve1 deletion strains in $\mathrm{V}$. dahliae with truncated versions of $\mathrm{VdAve1,}$ will reveal the particular involvement of the C-terminal nine amino acids in VdAve1 function.

Processing of the $\mathrm{VdAve} 1$ protein into smaller peptides might occur during infection. This is the case for the biologically active peptide in animal atrial natriuretic peptides (ANPs) which is a C-terminally derived 28 amino acid peptide that is cleaved from the 126 amino acid ANP precursor (Potter et al. 2009). The importance of precursor maturation has been demonstrated for the recognition of the CLV3 peptide by the eLRR-containing cell-surface receptor CLV2. Receptor CLV2 directly recognizes a 12 amino acid CLE motif of the Arabidopsis CLV3 peptide, that is also conserved in the nematode CLE-like GrCLE peptide, to modulate shoot apical meristem maintenance 
during development (Guo et al. 2011; Ni et al. 2011; Wang et al. 2010). Both types of CLE peptides are cleaved from their larger precursor proteins. Studies have suggested that maturation of the CLE peptide is essential for its function (Fiers et al. 2006; Fiers et al. 2005; Guo et al. 2011; Ni and Clark 2006). In addition, elongation of chemically synthesized CLE peptides was found to decrease its activity (Kondo et al. 2008; Kondo et al. 2006).

\section{Materials and methods}

\section{Plant materials}

Tobacco (Nicotiana tabacum cv. Petite Havana SR1) and transgenic tomato (Solanum lycopersicum cv. MoneyMaker p35S:Ve1) (Fradin et al. 2011) plants were grown in the greenhouse at $21^{\circ} \mathrm{C} / 19^{\circ} \mathrm{C}$ during $16 / 8$ hours day/night periods, respectively, with $70 \%$ relative humidity and $100 \mathrm{~W} / \mathrm{m}^{-2}$ supplemental light when the light intensity dropped below $150 \mathrm{~W} / \mathrm{m}^{-2}$. After agroinfiltration, tobacco plants were grown in the climate room at $22^{\circ} \mathrm{C} / 19^{\circ} \mathrm{C}$ during $16-\mathrm{h} / 8-\mathrm{h}$ day/night periods, respectively, with $70 \%$ relative humidity.

\section{Generation of expression constructs for Ave 1 homologs}

To generate constructs for constitutive expression of the Ave1 homologs, the coding sequences of the homologs were cloned into pDONR207 (Invitrogen, Carlsbad, California) through a Gateway BP reaction, and subsequently transferred into the Gateway-compatible destination vector pSol2092 (Zhang et al. 2013) to generate expression constructs driven by the constitutive CaMV35S promoter. VdAve1, VnAve1 and FoAve1 were amplified from cDNA using the primers attB$\mathrm{Vd}-\mathrm{F}$ and attB-Vd-R, attB- $\mathrm{Vn}-\mathrm{F}$ and attB- $\mathrm{Vn}-\mathrm{R}$, attB-Fo-F and attB-Fo-R, respectively (Supplemental Table 2). ChAve1, CbAve1, VvAve1, SIAve1and XacPNP were obtained by gene synthesis (Eurofins MWG Operon, Ebersberg, Germany).

\section{Generation of expression constructs for GFP-VdAve 1 fusion}

To generate VdAve1 fused at the C-terminus to the green fluorescent protein (GFP), the VdAve 1 coding sequence without stop codon was PCR amplified using primers containing Gateway attB sites (attB-Vd-F and attB-Vd-R-SC). The PCR fragment was cloned into pDONR207 (Invitrogen, Carlsbad, California) through a Gateway BP reaction to generate entry vector pDONR207::VdAve1-SC. Subsequently, pDONR207::VdAve1-SC was transferred into the Gateway-compatible destination vector pSol2095 (Zhang et al. 2013) to generate an expression construct driven by the constitutive CaMV35S promoter and C-terminally tagged to GFP. Similarly, 
pDONR207::VdAve1-SC was transferred into the Gateway-compatible destination vector pGWB14, pGWB8, pGWB17, pGWB20 and pGBW11 (Nakagawa et al. 2007) to generate VdAve1_HA, VdAve1_HIS, VdAve1_4MYC, VdAve1_10MYC, VdAve1_ FLAG, respectively. To generate VdAve1 fused at the N-terminus to a GFP tag, the GFP coding sequence lacking the stop codon was fused at the $\mathrm{N}$-terminus to the sequence encoding the signal peptide of $\mathrm{VdAve} 1$ to ensure extracellular targeting, and at the C-terminus to the VdAve1 coding sequence (without signal peptide). This construct was obtained by gene synthesis (Eurofins MWG Operon, Ebersberg, Germany), and subsequently cloned into Gateway destination vector pSol2092. Fusion constructs were transformed into Agrobacterium tumefaciens strain GV3101 by electroporation.

\section{Generation of deletion and domain swap constructs for VdAve 1}

Deletion constructs VdAve1_M1, VdAve1_M2, VdAve1_M3 and VdAve1_M4 were amplified from cDNA using the forward primers attB-Vd-F in combination with reverse primers attB-VdM1-R, attB-VdM2-R, attB-VdM3-R, and attB-VdM4-R, respectively. Vd_Ch9AA, Ch_Vd9AA, and SP_GFP-Vd9AA were amplified from cDNA using the primers attB-Vd-F and attB-VdCh9AA-R, attB-Ch- $F$ and attBChVd9AA-R, attB-Vd-F and attB-GFP-Vd9AA-R respectively (Supplemental Table 2). Vd_Xac9AA, Xac_Vd9AA and SP_Vd9AA were obtained by gene synthesis (Eurofins MWG Operon, Ebersberg, Germany). The AVNIEFRQI peptides were synthesized by GenScript company (Piscataway, USA; Purity $>75 \%$ ). The deletion and domain swap constructs were cloned into pDONR207 and subsequently transferred into pSol2092 to generate expression constructs.

\section{Generation of site-directed mutagenesis constructs for VdAve 1}

Site-directed mutagenesis constructs V127A, V127R, N128A, N128T, 1129A, E130A, E130P, F131A, F131W, R132A, Q133A, Q133N, I134A were amplified from pDONR207::VdAve_M4 plasmid using the forward primers attB-Vd-F in combination with corresponding reverse primers (Supplemental Table 2). The PCR products were cloned into pDONR207 and subsequently transferred into pSol2092 to generate expression constructs. Subsequently, pDONR207 carrying mutant constructs were transferred into pSol2092 for expression in planta.

\section{Agrobacterium tumefaciens-mediated transient expression}

Overnight cultures of A. tumefaciens strain GV3101 containing expression constructs were harvested at OD600 of 0.8 to 1 by centrifugation and resuspended to a final OD of 2 in infiltration medium as described previously (Zhang et al. 2013). 
A. tumefaciens cultures containing constructs to express VdAve1 and Ve1 proteins were mixed in a 1:1 ratio and infiltrated into leafs of five- to six-week-old tobacco plants. At five days post infiltration (dpi), necrosis was examined and quantified by measuring the area of necrosis as percentage of the total infiltrated area.

\section{Generation of Verticillium dahliae strains carrying VdAve 1 constructs}

pDONR207::VdAve1_M4 and pDONR207::SP_GFP-Vd9AA were used to clone the constructs into vector pFBT005 under the VdAve1 promoter, containing a nourseothricin cassette for selection. $V$. dahliae was transformed by $A$. tumefaciens carrying pFBT005 as previously described (Santhanam 2012) and selected on PDA plates containing nourseothricin sulphate (Sigma Aldrich Chemie BV, Zwijndrecht, The Netherlands). After five to seven days at room temperature, individual transformants were transferred to fresh PDA plates and incubated for ten days. Genomic DNA was extracted from the transformants and PCR was performed to test presence of the inserted nourseothricin cassette and of the inserted swap constructs.

\section{Verticillium inoculation}

For V. dahliae inoculations 10-day-old tomato plants were uprooted, rinsed in water and then dipped for 5 minutes in a suspension of $10^{6}$ conidia per $\mathrm{mL}$ of water harvested from 1- to 2-week-old V. dahliae cultures on PDA as previously described (Fradin et al. 2009). Control plants got the same treatment, but the roots were dipped in water without conidiospores. After replanting in fresh soil, plants were incubated at standard greenhouse conditions. Disease development was monitored up to $21 \mathrm{dpi}$. The canopy area of 8 plants was measured with ImageJ software and a One-Way ANOVA was performed with IBM SPSS statistics software.

\section{Generation of the structural model of VdAve 1}

The V. dahliae VdAve1 structure was predicted using I-TASSER v4.3 (Zhang 2008) and rendered using UCSF Chimera v1.10.1 (Pettersen et al. 2004). Structural predictions with C-Scores $>-1.5$ are generally considered to have a correct fold (C-Scores are typically in the range of $[-5,2]$ ) (Roy et al. 2010). The structural analog in the protein data bank (RCSB PDB) (Rose et al. 2013)was identified using the TM-align program which is part of the I-TASSER package. Analogous structures with TM-Scores $>0.5$ are considered to have a similar fold (TM-Scores in the range $[0,1]$ ) (Roy et al. 2010). 


\section{Acknowledgements}

B.P.H.J.T. is supported by a Vici grant of the Research Council for Earth and Life sciences (ALW) of the Netherlands Organization for Scientific Research (NWO). This research was funded in part by the Centre for BioSystems Genomics (CBSG) which is part of the Netherlands Genomics Initiative/NWO and by ERA-NET Plant Genomics. We thank Bert Essenstam and Pauline Sanderson at Unifarm for excellent plant care, and Sebastjan Radišek at the Institute of Hop Research and Brewing, Slovenia, for providing Verticillium strains.

\section{References}

Albert, I., Böhm, H., Albert, M., Feiler, C. E., Imkampe, J., Wallmeroth, N., Brancato, C., Raaymakers, T. M., Oome, S., Zhang, H., Krol, E., Grefen, C., Gust, A. A., Chai, J., Hedrich, R., Van den Ackerveken, G., and Nürnberger, T. 2015. An RLP23-SOBIR1-BAK1 complex mediates NLP-triggered immunity. Nat Plants 1:15140.

Böhm, H., Albert, I., Oome, S., Raaymakers, T. M., Van den Ackerveken, G., and Nürnberger, T. 2014. A conserved peptide pattern from a widespread microbial virulence factor triggers pattern-induced immunity in Arabidopsis. PLoS Pathog 10:e1004491.

Brunner, F., Rosahl, S., Lee, J., Rudd, J. J., Geiler, C., Kauppinen, S., Rasmussen, G., Scheel, D., and Nurnberger, T. 2002. Pep-13, a plant defense-inducing pathogen-associated pattern from Phytophthora transglutaminases. EMBO J 21:6681-6688.

Cai, R., Lewis, J., Yan, S., Liu, H., Clarke, C. R., Campanile, F., Almeida, N. F., Studholme, D. J., Lindeberg, M., and Schneider, D. 2011. The plant pathogen Pseudomonas syringae pv. tomato is genetically monomorphic and under strong selection to evade tomato immunity. PLoS Pathog 7:e1002130.

Ceroni, A., Passerini, A., Vullo, A., and Frasconi, P. 2006. DISULFIND: a disulfide bonding state and cysteine connectivity prediction server. Nucleic Acids Res 34:W177-181.

Chen, T., Kan, J., Yang, Y., Ling, X., Chang, Y., and Zhang, B. 2016. A Ve homologous gene from Gossypium barbadense, Gbvdr3, enhances the defense response against Verticillium dahliae. Plant Physiol Biochem 98:101-111.

Chou, S., Krasileva, K. V., Holton, J. M., Steinbrenner, A. D., Alber, T., and Staskawicz, B. J. 2011. Hyaloperonospora arabidopsidis ATR1 effector is a repeat protein with distributed recognition surfaces. Proc Natl Acad Sci U S A 108:13323-13328.

Cook, D. E., Mesarich, C. H., and Thomma, B. P. H. J. 2015. Understanding plant immunity as a surveillance system to detect invasion. Annu Rev Phytopathol.

de Jonge, R., van Esse, H. P., Maruthachalam, K., Bolton, M. D., Santhanam, P., Saber, M. K., Zhang, Z., Usami, T., Lievens, B., Subbarao, K. V., and Thomma, B. P. H. J. 2012. Tomato immune receptor Ve1 recognizes effector of multiple fungal pathogens uncovered by genome and RNA sequencing. Proc Natl Acad Sci U S A 109:5110-5115.

Dodds, P. N., and Rathjen, J. P. 2010. Plant immunity: towards an integrated view of plant-pathogen interactions. Nat Rev Genet 11:539-548.

Fiers, M., Golemiec, E., Xu, J., van der Geest, L., Heidstra, R., Stiekema, W., and Liu, C. M. 2005. The 14-amino acid CLV3, CLE19, and CLE40 peptides trigger consumption of the root meristem in Arabidopsis through a CLAVATA2-dependent pathway. Plant Cell 17:2542-2553.

Fiers, M., Golemiec, E., van der Schors, R., van der Geest, L., Li, K. W., Stiekema, W. J., and Liu, C. M. 2006. The CLAVATA3/ESR motif of CLAVATA3 is functionally independent from the nonconserved flanking sequences. Plant Physiol 141:1284-1292.

Flor, H. H. 1971. Current status of the gene-for-gene concept. Annu Rev Phytopathol 9:275-296. 
Fradin, E. F., and Thomma, B. P. H. J. 2006. Physiology and molecular aspects of Verticillium wilt diseases caused by V. dahliae and V. albo-atrum. Mol Plant Pathol 7:71-86.

Fradin, E. F., Abd-El-Haliem, A., Masini, L., van den Berg, G. C., Joosten, M. H., and Thomma, B. P. H. J. 2011. Interfamily transfer of tomato Ve1 mediates Verticillium resistance in Arabidopsis. Plant Physiol 156:2255-2265.

Fradin, E. F., Zhang, Z., Juarez Ayala, J. C., Castroverde, C. D., Nazar, R. N., Robb, J., Liu, C. M., and Thomma, B. P. H. J. 2009. Genetic dissection of Verticillium wilt resistance mediated by tomato Ve1. Plant Physiol 150:320-332.

Furukawa, T., Inagaki, H., Takai, R., Hirai, H., and Che, F.-S. 2014. Two distinct EF-Tu epitopes induce immune responses in rice and Arabidopsis. Mol. Plant Microbe Interact. 27:113-124.

Goritschnig, S., Steinbrenner, A. D., Grunwald, D. J., and Staskawicz, B. J. 2016. Structurally distinct Arabidopsis thaliana NLR immune receptors recognize tandem WY domains of an oomycete effector. New Phytol.

Gottig, N., Garavaglia, B. S., Daurelio, L. D., Valentine, A., Gehring, C., Orellano, E. G., and Ottado, J. 2008. Xanthomonas axonopodis pv. citri uses a plant natriuretic peptide-like protein to modify host homeostasis. Proc Natl Acad Sci U S A 105:18631-18636.

Guo, Y., Ni, J., Denver, R., Wang, X., and Clark, S. E. 2011. Mechanisms of molecular mimicry of plant CLE peptide ligands by the parasitic nematode Globodera rostochiensis. Plant Physiol 157:476-484.

Inderbitzin, P., Bostock, R. M., Davis, R. M., Usami, T., Platt, H. W., and Subbarao, K. V. 2011. Phylogenetics and taxonomy of the fungal vascular wilt pathogen Verticillium, with the descriptions of five new species. PLoS One 6:e28341.

Isaac, I. 1953. A further comparative study of pathogenic isolates of Verticillium: V. nubilum Pethybr. and V. tricorpus sp.nov. Trans Br Mycol Soc 36:180-IN182.

Jones, J. D., and Dangl, J. L. 2006. The plant immune system. Nature 444:323-329.

Kawchuk, L. M., Hachey, J., Lynch, D. R., Kulcsar, F., van Rooijen, G., Waterer, D. R., Robertson, A., Kokko, E., Byers, R., Howard, R. J., Fischer, R., and Prufer, D. 2001. Tomato Ve disease resistance genes encode cell surface-like receptors. Proc Natl Acad Sci U S A 98:6511-6515.

Kondo, T., Nakamura, T., Yokomine, K., and Sakagami, Y. 2008. Dual assay for MCLV3 activity reveals structure-activity relationship of CLE peptides. Biochem Biophys Res Commun 377:312-316.

Kondo, T., Sawa, S., Kinoshita, A., Mizuno, S., Kakimoto, T., Fukuda, H., and Sakagami, Y. 2006. A plant peptide encoded by CLV3 identified by in situ MALDI-TOF MS analysis. Science 313:845-848.

Naito, K., Taguchi, F., Suzuki, T., Inagaki, Y., Toyoda, K., Shiraishi, T., and Ichinose, Y. 2008. Amino acid sequence of bacterial microbe-associated molecular pattern flg22 is required for virulence. Mol. Plant Microbe Interact. 21:1165-1174.

Nakagawa, T., Suzuki, T., Murata, S., Nakamura, S., Hino, T., Maeo, K., Tabata, R., Kawai, T., Tanaka, K., Niwa, Y., Watanabe, Y., Nakamura, K., Kimura, T., and Ishiguro, S. 2007. Improved gateway binary vectors: High-performance vectors for creation of fusion constructs in Transgenic analysis of plants. Biosci Biotechnol Biochem 71:2095-2100.

Nembaware, V., Seoighe, C., Sayed, M., and Gehring, C. 2004. A plant natriuretic peptide-like gene in the bacterial pathogen Xanthomonas axonopodis may induce hyper-hydration in the plant host: a hypothesis of molecular mimicry. BMC Evol Biol 4:10.

$\mathrm{Ni}$, J., and Clark, S. E. 2006. Evidence for functional conservation, sufficiency, and proteolytic processing of the CLAVATA3 CLE domain. Plant Physiol 140:726-733.

Ni, J., Guo, Y., Jin, H., Hartsell, J., and Clark, S. E. 2011. Characterization of a CLE processing activity. Plant Mol Biol 75:67-75.

Oome, S., Raaymakers, T. M., Cabral, A., Samwel, S., Böhm, H., Albert, I., Nürnberger, T., and Van den Ackerveken, G. 2014. Nep1-like proteins from three kingdoms of life act as a microbe-associated molecular pattern in Arabidopsis. Proc Natl Acad Sci U S A 111:16955-16960.

Pearce, G., Yamaguchi, Y., Munske, G., and Ryan, C. A. 2008. Structure-activity studies of AtPep1, a plant peptide signal involved in the innate immune response. Peptides 29:2083-2089. 
Petersen, T. N., Brunak, S., von Heijne, G., and Nielsen, H. 2011. SignalP 4.0: discriminating signal peptides from transmembrane regions. Nat Methods 8:785-786.

Pettersen, E. F., Goddard, T. D., Huang, C. C., Couch, G. S., Greenblatt, D. M., Meng, E. C., and Ferrin, T. E. 2004. UCSF Chimera - A visualization system for exploratory research and analysis. J Comput Chem 25:1605-1612.

Potter, L. R., Yoder, A. R., Flora, D. R., Antos, L. K., and Dickey, D. M. 2009. Natriuretic peptides: their structures, receptors, physiologic functions and therapeutic applications. 2008/12/18 ed.

Pruitt, R. N., Schwessinger, B., Joe, A., Thomas, N., Liu, F., Albert, M., Robinson, M. R., Chan, L. J. G., Luu, D. D., Chen, H., Bahar, O., Daudi, A., De Vleesschauwer, D., Caddell, D., Zhang, W., Zhao, X., Li, X., Heazlewood, J. L., Ruan, D., Majumder, D., Chern, M., Kalbacher, H., Midha, S., Patil, P. B., Sonti, R. V., Petzold, C. J., Liu, C. C., Brodbelt, J. S., Felix, G., and Ronald, P. C. 2015. The rice immune receptor XA21 recognizes a tyrosine-sulfated protein from a Gram-negative bacterium. Sci Adv 1:e1500245.

Ron, M., and Avni, A. 2004. The receptor for the fungal elicitor ethylene-inducing xylanase is a member of a resistance-like gene family in tomato. Plant Cell 16:1604-1615.

Rose, P. W., Bi, C., Bluhm, W. F., Christie, C. H., Dimitropoulos, D., Dutta, S., Green, R. K., Goodsell, D. S., Prlić, A., Quesada, M., Quinn, G. B., Ramos, A. G., Westbrook, J. D., Young, J., Zardecki, C., Berman, H. M., and Bourne, P. E. 2013. The RCSB Protein Data Bank: new resources for research and education. Nucleic Acids Res 41:D475-D482.

Rotblat, B., Enshell-Seijffers, D., Gershoni, J. M., Schuster, S., and Avni, A. 2002. Identification of an essential component of the elicitation active site of the EIX protein elicitor. Plant J 32:1049-1055.

Roy, A., Kucukural, A., and Zhang, Y. 2010. I-TASSER: a unified platform for automated protein structure and function prediction. Nat Protoc 5:725-738.

Santhanam, P. 2012. Random insertional mutagenesis in fungal genomes to identify virulence factors. Pages 509-517 in: Plant fungal pathogens. Methods in molecular biology, vol. 835. T. B. Bolton MD, ed. Humana press, Totowa.

Schaible, L., Cannon, O. S., and Waddoups, V. 1951. Inheritance of resistance to Verticillium wilt in a tomato cross. Phytopathology 41:986-990.

Song, X. F., Yu, D. L., Xu, T. T., Ren, S. C., Guo, P., and Liu, C. M. 2012. Contributions of individual amino acid residues to the endogenous CLV3 function in shoot apical meristem maintenance in Arabidopsis. Mol Plant 5:515-523.

Thomma, B. P. H. J., Nurnberger, T., and Joosten, M. H. A. J. 2011. Of PAMPs and effectors: The blurred PTI-ETI dichotomy. Plant Cell 23:4-15.

van Kan, J. A., van den Ackerveken, G., and de Wit, P. J. G. M. 1991. Cloning and characterization of cDNA of avirulence gene avr9 of the fungal pathogen Cladosporium fulvum, causal agent of tomato leaf mold. Mol. Plant Microbe Interact. 4:52-59.

Ve, T., Williams, S. J., Catanzariti, A.-M., Rafiqi, M., Rahman, M., Ellis, J. G., Hardham, A. R., Jones, D. A., Anderson, P. A., and Dodds, P. N. 2013. Structures of the flax-rust effector AvrM reveal insights into the molecular basis of plant-cell entry and effector-triggered immunity. Proc Natl Acad Sci U S A 110:17594-17599.

Wang, C.-I. A., Gunčar, G., Forwood, J. K., Teh, T., Catanzariti, A.-M., Lawrence, G. J., Loughlin, F. E., Mackay, J. P., Schirra, H. J., and Anderson, P. A. 2007. Crystal structures of flax rust avirulence proteins AvrL567-A and-D reveal details of the structural basis for flax disease resistance specificity. Plant Cell 19:2898-2912.

Wang, G., Fiers, M., Ellendorff, U., Wang, Z. Z., de Wit, P. J. G. M., Angenent, G. C., and Thomma, B. P. H. J. 2010. The diverse roles of extracellular leucine-rich repeat-containing receptor-like proteins in plants. CRC Crit Rev Plant Sci 29:285-299.

Yang, Y., Ling, X., Chen, T., Cai, L., Liu, T., Wang, J., Fan, X., Ren, Y., Yuan, H., and Zhu, W. 2014. A cotton Gbvdr5 gene encoding a leucine-rich-repeat receptor-like protein confers resistance to Verticillium dahliae in transgenic Arabidopsis and upland cotton. Plant Mol Biol Report:1-15. 
Yennawar, N. H., Li, L. C., Dudzinski, D. M., Tabuchi, A., and Cosgrove, D. J. 2006. Crystal structure and activities of EXPB1 (Zea $m$ 1), a beta-expansin and group-1 pollen allergen from maize. Proc Natl Acad Sci U S A 103:14664-14671.

Zhang, B., Yang, Y., Chen, T., Yu, W., Liu, T., Li, H., Fan, X., Ren, Y., Shen, D., Liu, L., Dou, D., and Chang, Y. 2012. Island cotton Gbve1 gene encoding a receptor-like protein confers resistance to both defoliating and non-defoliating isolates of Verticillium dahliae. PLoS One 7:e51091.

Zhang, Y. 2008. I-TASSER server for protein 3D structure prediction. BMC Bioinformatics 9:40.

Zhang, Y., Wang, X., Yang, S., Chi, J., Zhang, G., and Ma, Z. 2011. Cloning and characterization of a Verticillium wilt resistance gene from Gossypium barbadense and functional analysis in Arabidopsis thaliana. Plant Cell Rep 30:2085-2096.

Zhang, Z., Fradin, E., de Jonge, R., van Esse, H. P., Smit, P., Liu, C.-M., and Thomma, B. P. H. J. 2013. Optimized agroinfiltration and virus-induced gene silencing to study Ve1-mediated Verticillium resistance in tobacco. Mol. Plant Microbe Interact. 26:182-190.

Zipfel, C., Robatzek, S., Navarro, L., Oakeley, E. J., Jones, J. D. G., Felix, G., and Boller, T. 2004. Bacterial disease resistance in Arabidopsis through flagellin perception. Nature 428:764-767.

Zipfel, C., Kunze, G., Chinchilla, D., Caniard, A., Jones, J. D., Boller, T., and Felix, G. 2006. Perception of the bacterial PAMP EF-Tu by the receptor EFR restricts Agrobacterium-mediated transformation.

Cell 125:749-760.

\section{Supplemental data}

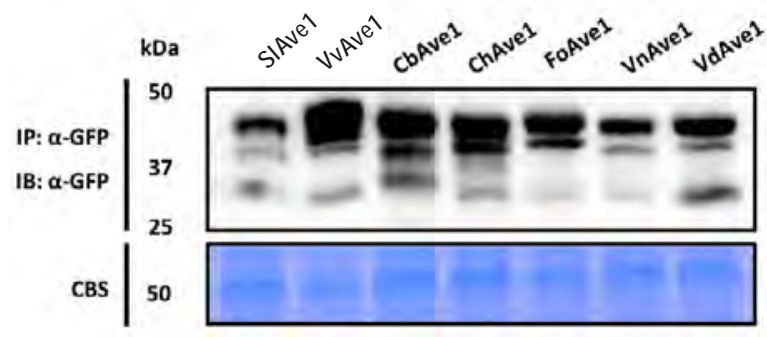

SUPPLEMENTAL FIGURE 1 | Immunoblotting of GFP-tagged Ave1 homologs. GFP-tagged Ave1 homologs were detected by western blotting using GFP antibody (a-GFP). Coomassie-stained blots (CBS) showing the $50 \mathrm{kDa}$ Rubisco band present in the input samples confirm equal loading.

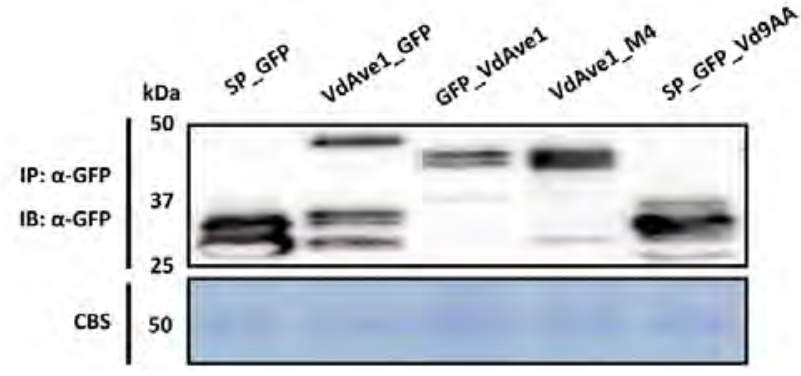

SUPPLEMENTAL FIGURE 2 |Stability of truncated Ave1 proteins. SP_GFP, VdAve1_GFP, GFP_VdAve1, GFP-tagged VdAve1_M4 and SP_GFP_Vd9AA proteins were detected using GFP antibody (a-GFP). Coomassie-stained blots (CBS) showing the $50 \mathrm{kDa}$ Rubisco band present in the input samples confirm equal loading. 


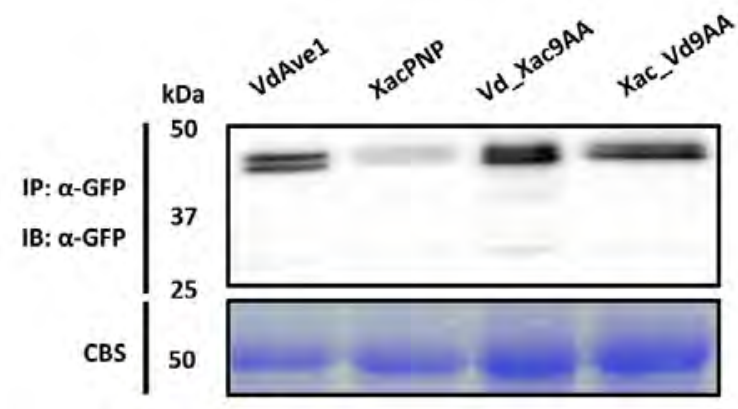

SUPPLEMENTAL FIGURE 3 | Stability of chimeric Ave proteins Vd_Xac9AA and Xac_Vd9AA. GFP-tagged VdAve1, XacPNP, chimeric constructs Vd_Xac9AA and Xac_Vd9AA were detected by immunoblotting using GFP antibody ( $a$-GFP). Coomassie-stained blots (CBS) showing the $50 \mathrm{kDa}$ Rubisco band present in the input samples confirm equal loading.

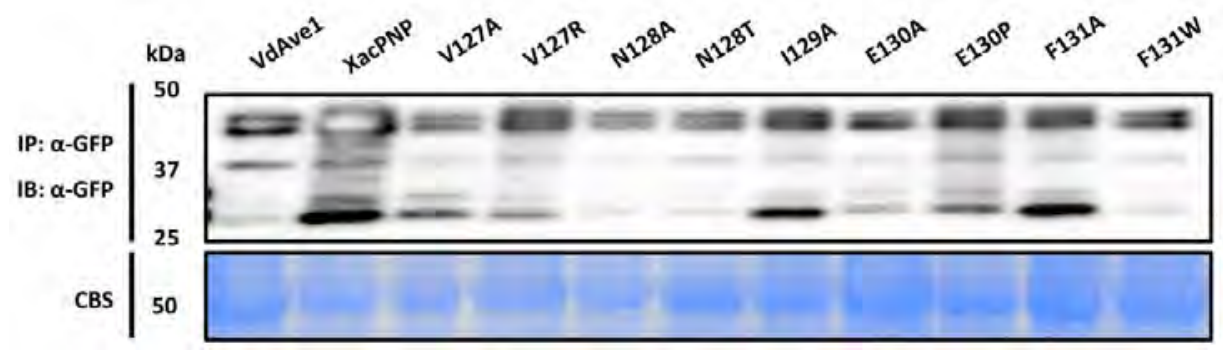

SUPPLEMENTAL FIGURE 4 | Stability of site-directed mutagenesis constructs that showed compromised HR-inducing capacity. GFP-tagged VdAve1, XacPNP, site-directed mutagenesis constructs V127A, V127R, N128A, N128T, I129A, E130A, F131A, F131W, R132A, Q133A, I134A were detected by immunoblotting using GFP antibody ( $a$-GFP). Coomassie-stained blots (CBS) showing the $50 \mathrm{kDa}$ Rubisco band present in the input samples confirm equal loading.

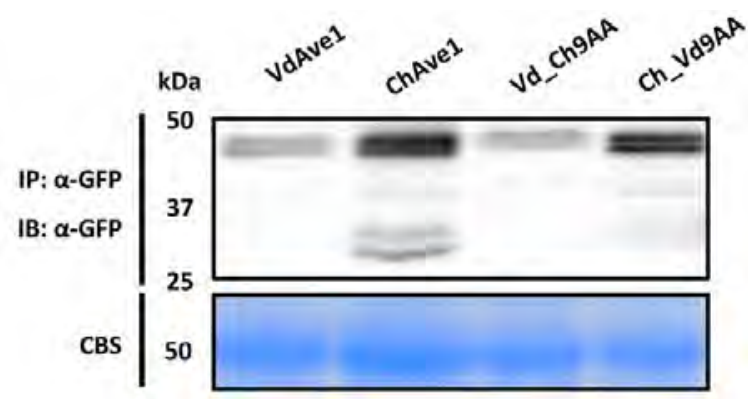

SUPPLEMENTAL FIGURE 5 | Stability of chimeric Ave proteins Vd_Ch9AA and Ch_Vd9AA. GFP-tagged VdAve1, ChAve1, chimeric constructs Vd_Ch9AA and Ch_Vd9AA were detected by immunoblotting using GFP antibody. Coomassie-stained blots (CBS) showing the $50 \mathrm{kDa}$ Rubisco band present in the input samples confirm equal loading. 
SUPPLEMENTAL TABLE 1 | Verticillium strains analysed for presence of Ave1 homologs

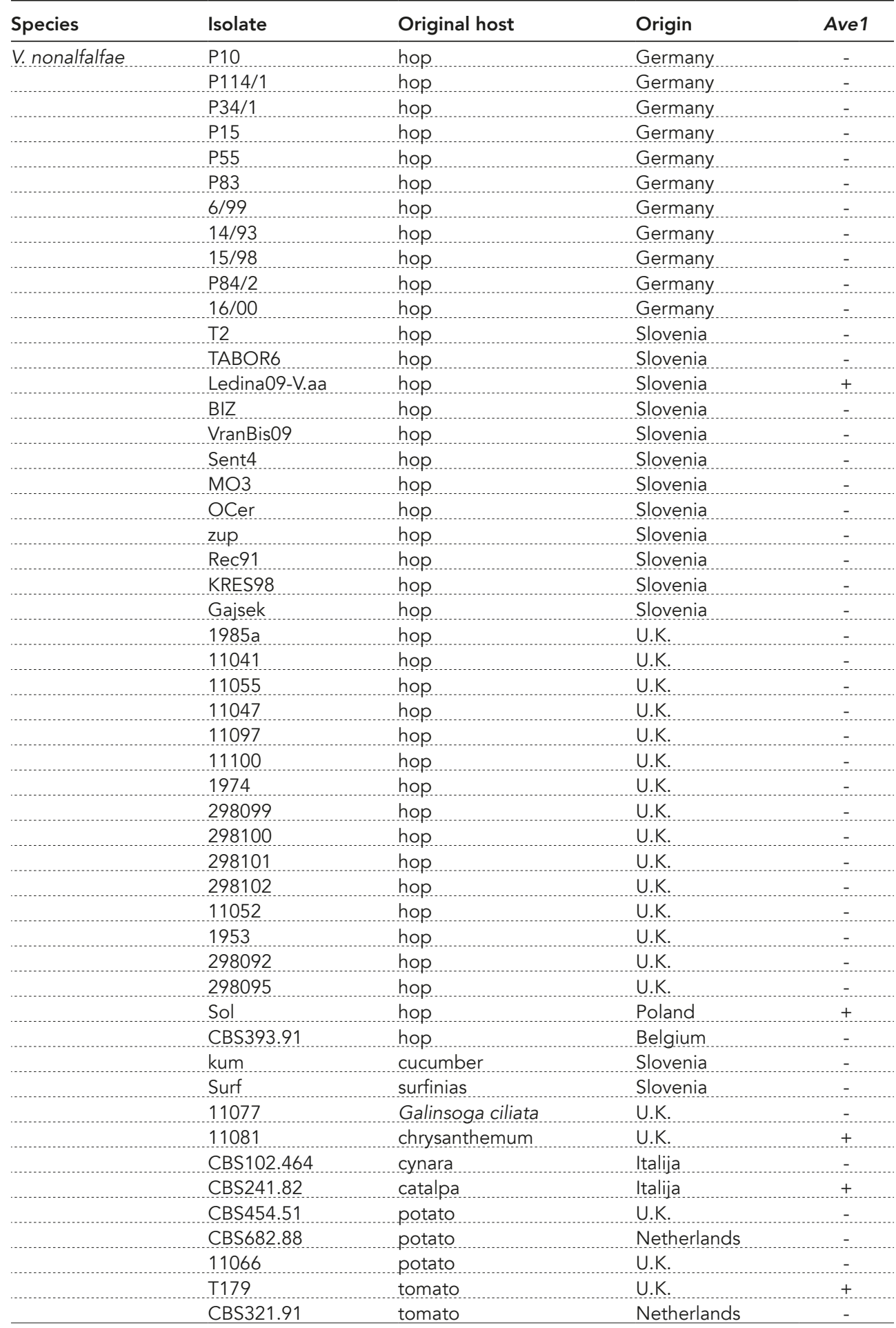




\begin{tabular}{|c|c|c|c|c|}
\hline Species & Isolate & Original host & Origin & Ave1 \\
\hline & AR01/067 & tomato & U.K. & - \\
\hline & ARO/140 & tomato & U.K. & - \\
\hline & AR01/JS1 & tomato & U.K. & - \\
\hline & $\mathrm{PD} 83 / 53 \mathrm{a}$ & tomato & Netherlands & - \\
\hline & PD2000/4186a & tomato & Netherlands & + \\
\hline \multirow[t]{8}{*}{ V. alfalfae } & LuC & alfalfa & U.K. & + \\
\hline & 41 & alfalfa & Canada & - \\
\hline & CBS392.91 & alfalfa & Netherlands & - \\
\hline & 107 & alfalfa & USA & - \\
\hline & PD693 & potato & Iran & - \\
\hline & 314193 & potato & Australia & - \\
\hline & 340646 & potato & Spain & + \\
\hline & 11 & alfalfa & Slovenia & - \\
\hline \multirow[t]{24}{*}{ V. dahliae } & JKG2 & catalpa & Netherlands & - \\
\hline & CIG3-Vd & hop & Slovenia & - \\
\hline & JKG1 & potato & Netherlands & - \\
\hline & JKG8 & potato & Netherlands & - \\
\hline & A56 & potato & Slovenia & + \\
\hline & DJK & chrysanthemum & Netherlands & - \\
\hline & $\mathrm{MH}$ & chrysanthemum & Netherlands & - \\
\hline & Mint & mint & USA & - \\
\hline & GAJO9 & hop & Slovenia & - \\
\hline & PDRENU & hop & Slovenia & - \\
\hline & CasD & hop & Slovenia & - \\
\hline & KresD & hop & Slovenia & - \\
\hline & MoD & hop & Slovenia & - \\
\hline & Oset & hop & Slovenia & - \\
\hline & 12099 & hop & U.K. & - \\
\hline & 12042 & hop & U.K. & - \\
\hline & PD335 & cabbage & unknown & - \\
\hline & PD584 & $\operatorname{mint}$ & unknown & - \\
\hline & $V-1761$ & cotton & Netherlands & + \\
\hline & $\mathrm{V}-1381$ & cotton & Germany & - \\
\hline & PAPmb & pepper & Slovenia & - \\
\hline & PAP & pepper & Slovenia & + \\
\hline & Pap99 & pepper & Slovenia & + \\
\hline & Pap2008 & pepper & Slovenia & + \\
\hline \multirow[t]{4}{*}{ V. nubilum } & CBS456.51 & potato & UK & + \\
\hline & CBS457.51 & soil & UK & + \\
\hline & PD621 & mushroom compost & UK & + \\
\hline & PD702 & potato & UK & + \\
\hline \multirow[t]{2}{*}{ V. tricorpus } & CBS227.84 & potato & Netherlands & $\therefore$ \\
\hline & JKG2O & linden & Netherlands & - \\
\hline \multirow[t]{2}{*}{ V. longisporium } & CBS110218 & Brassica napus & Sweden & - \\
\hline & PD330 & cabbage & unknown & - \\
\hline
\end{tabular}


SUPPLEMENTAL TABLE 2 | Primers used in this study.

\begin{tabular}{|c|c|}
\hline Primer name & Sequence $\left(5^{\prime}-3^{\prime}\right)$ \\
\hline attB-Vd-F & GGGGACAAGTTTGTACAAAAAAGCAGGCTATGAAGCTTTCTACGCTT \\
\hline attB-Vd-R & GGGGACCACTTTGTACAAGAAAGCTGGGTTTATATCTGTCTAAATTC \\
\hline attB-Vn-F & GGGGACAAGTTTGTACAAAAAAGCAGGCTATGAAGCTTTCTACGCTT \\
\hline attB-Vn-R & GGGGACCACTTTGTACAAGAAAGCTGGGTTTATATCTGTTCAAACTC \\
\hline attB-Fo-F & GGGGACAAGTTTGTACAAAAAAGCAGGCTATGAAACTACTCGCACTA \\
\hline attB-Fo-R & GGGGACCACTTTGTACAAGAAAGCTGGGTTCATCTTTGTACAAAATCGATATTT \\
\hline attB-Vd-R-SC & GGGGACCACTTTGTACAAGAAAGCTGGGTCTATCTGTCTAAATTC \\
\hline attB-VdM1-R & GGGGACCACTTTGTACAAGAAAGCTGGGTTTACTTATGCCTCGTTCCCTT \\
\hline attB-VdM2-R & GGGGACCACTTTGTACAAGAAAGCTGGGTTTAAACAATGGCATCATATGAGT \\
\hline attB-VdM3-R & GGGGACCACTTTGTACAAGAAAGCTGGGTTTACTTGTGTGCTGCTTTGGTAA \\
\hline attB-VdM4-R & GGGGACCACTTTGTACAAGAAAGCTGGGTTTAAGCTCTGTCAACCACCCGCA \\
\hline attB-VdCh9AA-R & $\begin{array}{l}\text { GGGGACCACTTTGTACAAGAAAGCTGGGTTCAAATTTGTACGTACTCAATGTTTACTTTCT- } \\
\text { TATGCCTCGTTCCCTT }\end{array}$ \\
\hline attB-Ch-F & GGGGACAAGTTTGTACAAAAAAGCAGGCTATGAAGGCCTTCTTTCTAGGAG \\
\hline attB-ChVd9AA-R & $\begin{array}{l}\text { GGGGACCACTTTGTACAAGAAAGCTGGGTTTATATCTGTCTAAATTCGATGTTGACC- } \\
\text { GCAGTTACGCCAAAGTCCG }\end{array}$ \\
\hline attB-GFP-Vd9AA-R & $\begin{array}{l}\text { GGGGACCACTTTGTACAAGAAAGCTGGGTTTATATCTGTCTAAATTCGATGTTGACCGC } \\
\text { TTTGTATAGTTCATCCATGC }\end{array}$ \\
\hline V127A R & TTATATCTGTCTAAATTCGATGTTCGCCGCCTTATGCCTCGTTCCCTTC \\
\hline N128A R & TTATATCTGTCTAAATTCGATCGCGACCGCCTTATGCCTCGTTCCCTTC \\
\hline I129A R & TTATATCTGTCTAAATTCCGCGTTGACCGCCTTATGCCTCGTTCCCTTC \\
\hline E130A R & TTATATCTGTCTAAACGCGATGTTGACCGCCTTATGCCTCGTTCCCTTC \\
\hline F131A R & TTATATCTGTCTCGCTTCGATGTTGACCGCCTTATGCCTCGTTCCCTTC \\
\hline R132A R & TTATATCTGCGCAAATTCGATGTTGACCGCCTTATGCCTCGTTCCCTTC \\
\hline Q133A R & TTATATCGCTCTAAATTCGATGTTGACCGCCTTATGCCTCGTTCCCTTC \\
\hline I134A R & TTACGCCTGTCTAAATTCGATGTTGACCGCCTTATGCCTCGTTCCCTTC \\
\hline V127R R & TTATATCTGTCTAAATTCGATGTTTCTCGCCTTATGCCTCGTTCCCTTC \\
\hline N128T R & TTATATCTGTCTAAATTCGATTGTGACCGCCTTATGCCTCGTTCCCTTC \\
\hline E130P R & TTATATCTGTCTAAAGGGGATGTTGACCGCCTTATGCCTCGTTCCCTTC \\
\hline F131W R & TTATATCTGTCTCCATTCGATGTTGACCGCCTTATGCCTCGTTCCCTTC \\
\hline Q133NR & ТTATATATTTCTAAATTCGATGTTGACCGCCTTATGCCTCGTTCCCTTC \\
\hline $\mathrm{Vd} \_\mathrm{Xac} \_\mathrm{V} 2 \mathrm{R}$ & TTAAATATTTGCCCAGGGCGCTGTGACTGCCTTATGCCTCGTTCCCTTC \\
\hline $\mathrm{Vd} \mathrm{Xac}_{-} \mathrm{V} 5 \mathrm{R}$ & TTAAATATTTGCCCATTCCGCTGTTCTTGCCTTATGCCTCGTTCCCTTC \\
\hline $\mathrm{Vd} \mathrm{Xac}_{2} \mathrm{~V} 2 \mathrm{~V} 5 \mathrm{R}$ & TTAAATATTTGCCCATTCCGCTGTGACTGCCTTATGCCTCGTTCCCTTC \\
\hline Vd_Xac_V2V6 R & TTAAATATTTGCAAATTCCGCGTTGACTGCCTTATGCCTCGTTCCCTTC \\
\hline
\end{tabular}



Characterization of an expanded family of Ave 1 homologs in the apple and pear scab fungi

Venturia inaequalis and V. pirina

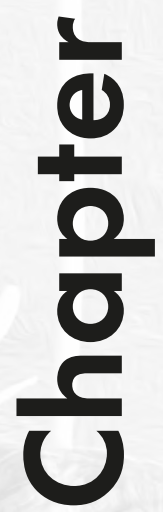


Venturia inaequalis and V. pirina are fungal pathogens that cause scab on apple and pear, respectively. Although several gene-forgene interactions have been identified for $V$. inaequalis and apple, no avirulence gene has been cloned thus far. Recently, a family of Ave1 homologs was identified in V. pirinia. Ave1 was first identified in race 1 strains of Verticillium dahliae that has a wide host range including many crops, and was shown to be recognized by the tomato Ve1 immune receptor. In absence of Ve1, Ave1 contributes to virulence of $V$. dahliae. Homologs of Ave1 have been identified in a wide range of plants and in a handful of phylogenetically unrelated plant pathogens, including the fungi Fusarium oxysporum, Cercospora beticola and Colletotrichum higginsianum and the bacterium Xanthomonas axonopodis. In this study we found that also $\mathrm{V}$. inaequalis contains a family of Ave 1 homologs. We show that the tomato Ve1 immune receptor is unable to recognize a selection of Ave1 homologs from $V$. inaequalis and $V$. pirina. Transcriptome analysis revealed that $V$. inaequalis ViAve1-1, ViAve1-5 and ViAve1-9 are expressed in planta. We show that in planta expressed ViAve1-1, ViAve1-5 and ViAve1-9 are unable to complement a $V$. dahliae VdAve1 deletion strain, suggesting that these homologs have different functions than $\mathrm{V}$. dahliae VdAve1. 


\section{Introduction}

Plants evolved an immune system that detects conserved microbe-associated molecular patterns (MAMPS) to activate defense responses against invading pathogens (Cook et al. 2015). Successful plant pathogens evolved appropriate effectors; secreted molecules of which many can deregulate immunity in the respective host (Rovenich et al. 2014) and that are typically species, strain or even isolate specific. In turn, host plants typically evolve immune receptors that can recognize such effectors and mount an immune response to prevent further invasion. However, recognition of effectors and subsequent immune responses pose a huge selection pressure on pathogens to either evolve new effectors or alter or loose recognized effectors to prevent the activation of immune responses. This continuous co-evolution between host and pathogen is elegantly visualized in the zig-zag model (Jones and Dangl 2006). However, this model is focused from the point-of-view of the plant, while the point-of-view of the pathogen is largely underrepresented. Additionally, effectors and MAMPs are depicted as two distinct and opposing features of pathogen molecules, while this distinction is often not clear cut (Thomma et al. 2011). The recently proposed invasion model states that any signal that betrays an invading organism can potentially serve as a cue to activate an appropriate host response, either to a beneficial or detrimental organism (Cook et al. 2015).

Verticillium dahliae is a fungal plant pathogen with a broad host range that includes many crops (Fradin and Thomma 2006). V. dahliae race 1 strains express the effector Ave1 that activates immunity in tomato plants carrying the Ve1 receptor (de Jonge et al. 2012). In addition, it was shown that Ave1 contributes to the virulence of V. dahliae. Homologs of Ave1 are mainly found in plants and only in a handful of plant pathogens, including the fungi Fusarium oxysporum, Cercospora beticola and Colletotrichum higginsianum and the bacterium Xanthomonas axonopodis. Only one homolog of Ave1 is found in the genome of each of these organisms, and these homologs share between $52-95 \%$ sequence similarity. In contrast, each V. dahliae strain analyzed thus far contains an additional Ave1-like (Ave1L) allele. Five alleles have been identified, denoted as Ave 1 L 1 to Ave 1 L5 that share between 64 and 68\% sequence similarity with V. dahliae VdAve1 (Chapter 6, this thesis). Co-expression of Ave1 homologs derived from Fusarium oxysporum, Cercospora beticola and Colletotrichum higginsianum and Xanthomonas axonopodis with tomato immune receptor Ve1 revealed differential recognition of the various homologs (de Jonge et al. 2012). 
Venturia inaequalis and V. pirina cause devastating scab diseases on apple and pear, respectively (Bowen et al. 2011; Shabi et al. 1973). Although a number of gene-forgene interactions have been identified for $V$. inaequalis and its host apple (Bus et al. 2011; Gessler et al. 2006), no avirulence genes have been cloned thus far (Bus et al. 2011). AvrLm6 is an effector protein of the fungal pathogen Leptosphaeria maculans that triggers an immune response in Brassica plants expressing the immune receptor Rlm6 (Fudal et al. 2007). Homologs of AvrLm6 have been identified in other plant pathogens, such as Colletotrichum and Fusarium species (Grandaubert et al. 2014). Interestingly, an expanded family of AvrLm6 effector homologs was identified in the genomes of $V$. inaequalis and $V$. pirina (Shiller et al. 2015). However, the $V$. inaequalis AvrLm6 homolog with the highest sequence similarity to L. maculans AvrLm6 was unable to trigger an immune response in Brassica plants expressing the immune receptor Rlm6 (Cooke et al. 2014). Recently it was found that the $V$. pirina genomes also contains an expanded Ave 1 effector gene family, which is in contrast to the plant pathogens Fusarium oxysporum, Cercospora beticola and Colletotrichum higginsianum and Xanthomonas axonopodis that carry only one homolog of Ave1 (Cooke et al. 2014; de Jonge et al. 2012). In this study we test whether any of the homologs in the expanded Ave 1 family derived from Venturia spp. is recognized by the tomato $V e 1$ immune receptor.

\section{Results}

\section{Ave 1 homologs derived from Venturia inaequalis and $V$. pirina are not recognized by tomato Ve 1}

Previously, 14 loci with homology to $V$. dahliae VdAve1 were identified in the genome of $V$. pirina, including 12 genes (VpAve1-1 to VpAve1-12) and 2 pseudogenes (VpAve1-13 and VpAve1-14) (Cooke et al. 2014) (Joanna Bowen personal communication). We similarly analysed the genome of $V$. inaequalis for the presence of Ave1 homologs. In this analysis, 17 loci were identified comprising 9 genes (ViAve1-1 to ViAve1-5 and ViAve1-8 to ViAve1-10) and 8 pseudogenes (ViAve1-6, ViAve1-7 and ViAve1-11 to ViAve1-16) (Kim Plummer personal communication). The Ave1 homologs derived from V. inaequalis and V. pirina display between 51-63\% sequence similarity to $V$. dahliae Ave1 (VdAve1). Alignment of the gene products of $V$. inaequalis and $V$. pirina Ave 1 homologs with $V$. dahliae $V d A v e 1$ shows a high degree of sequence conservation distributed over the whole protein sequence (Figure 1). We hypothesized that since Ave1 is recognized by tomato immune receptor Ve1, Venturia Ave1 homologs are potentially recognized by Ve1 as well. To test this hypothesis, we co-expressed Ve1 with Ave1 homologs from $V$. inaequalis (ViAve1-1, ViAve1-2, ViAve1-4, ViAve1-5, ViAve1-8, ViAve1-9, ViAve1-10), and Ave1 
homologs from V. pirina (VpAve1-1, VpAve1-2, VpAve1-3, VpAve1-4, VpAve1-5, VpAve1-6, VpAve1-7, VpAve1-11). Unfortunately, we were unable to clone Ave1 homologs ViAve1-3, VpAve1-8, VpAve1-9, VpAve1-10 and VpAve1-12. Whereas at 5 days post inoculation (dpi) a clear hypersensitive response (HR) was observed upon co-expression of the control combination of Ve1 and V. dahliae Ave1, no HR was observed in any of the co-inoculations of $V e 1$ with the selected $V$. inaequalis and $V$. pirina Ave1 homologs, except for some small necrotic spots in the case of ViAve1-4 and ViAve1-9 (Figure 2A and B). These results suggest that the tomato immune receptor $V e 1$ is unable to recognize Ave1 homologs derived from the apple and pear scab fungi $V$. inaequalis and $V$. pirina, respectively.

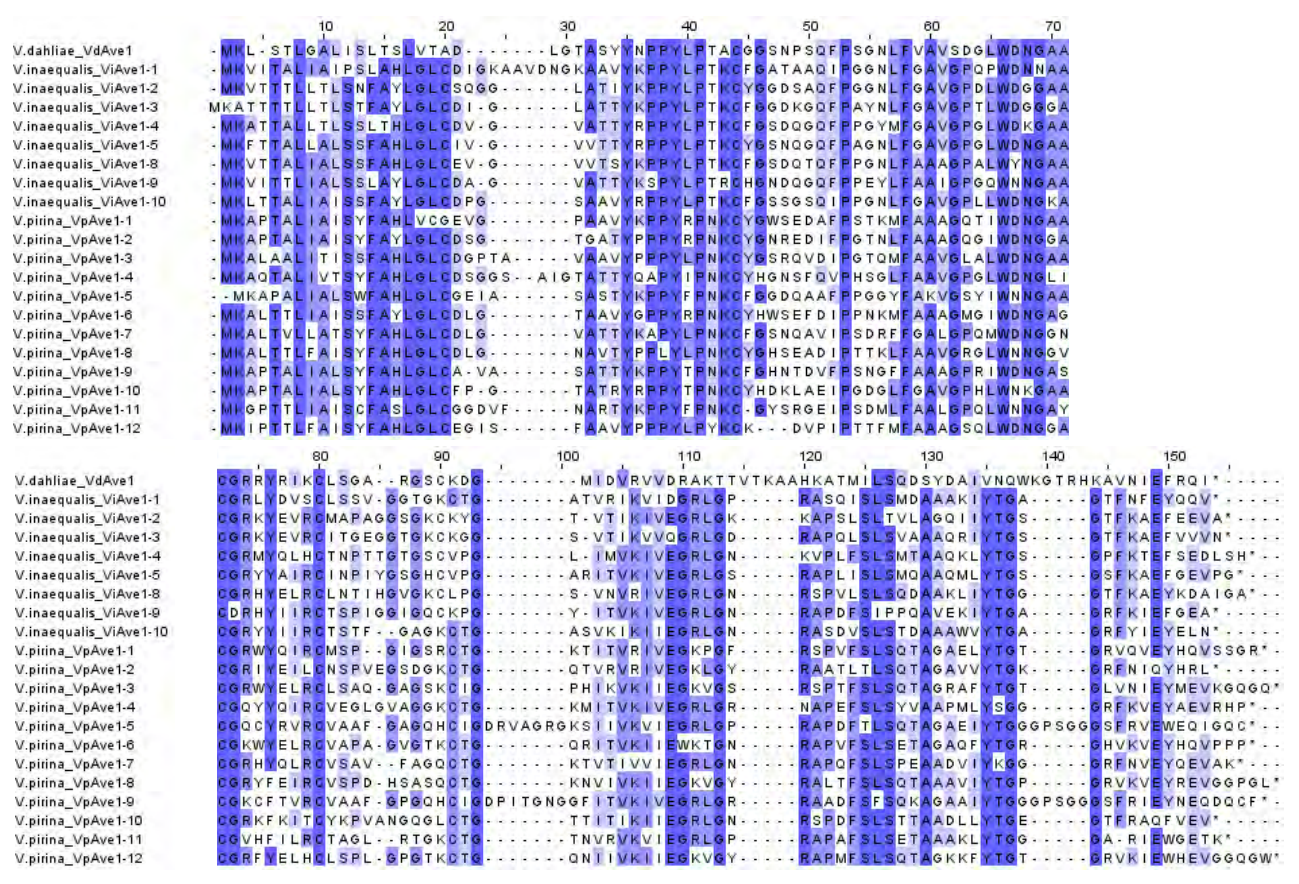

FIGURE 1 | Alignment of Ave1 homologs indicates regions with high conservation Alignment of Ave1 homologs derived from Venturia inaequalis and V. pirina with V. dahliae VdAve1. The intensity of the blue color indicates the degree of conservation. 


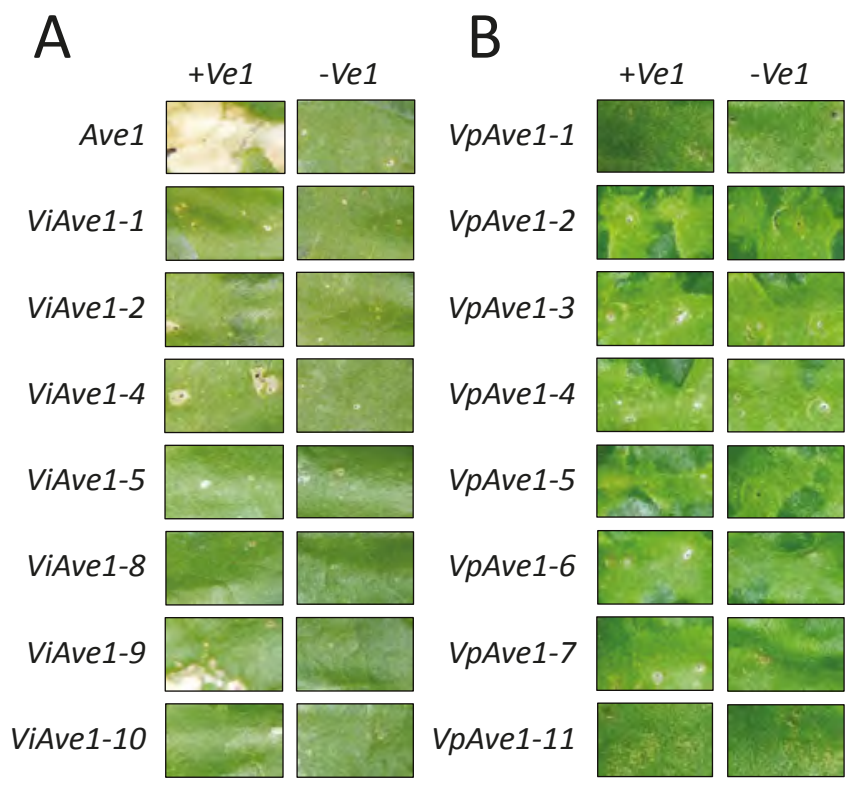

FIGURE 2 | Ave1 homologs derived from Venturia inaequalis and V. pirina are not recognized by tomato Ve1 (A) Co-expression of selected Ave1 homologs from Venturia inequalis with tomato Ve1. (B) Co-expression of selected Ave1 homologs from Venturia pirina with tomato Ve1.

\section{Ave 1 homologs derived from Venturia inaequalis cannot complement loss of Verticillium dahliae Ave 1}

A method to mimic leaf or fruit surface penetration and in planta expression is to grow $V$. inaequalis on cellophane discs. Therefore, transcriptome analysis was performed on V. inaequalis grown on cellophane discs (Kucheryava et al. 2008). This analysis revealed that the Ave1 homologs ViAve1-1, ViAve1-5 and ViAve1-9 are induced in planta (Kim Plummer personal communication). To test whether the Ave1 homologs from $V$. inaequalis share the same functionality as $V$. dahliae Ave 1 to promote fungal virulence, we transformed a $V$. dahliae Ave 1 deletion strain with constructs to express ViAve1-1, ViAve1-5 and ViAve1-9 driven by the V. dahliae Ave1 promotor. After confirmation that the strains contain the expression construct, the transformants were inoculated onto tomato plants that lack the Ve1 immune receptor and screened for virulence. Tomato plants that were inoculated with wild type $V$. dahliae and the V. dahliae Ave1 deletion strain that was complemented with VdAve1 as a control showed clear symptoms of Verticillium wilt. In contrast, the Ave1 deletion strain and the transformants carrying expression constructs for ViAve1-1, ViAve1-5 and ViAve1-9 only induced reduced Verticillium wilt symptoms when compared with the symptoms induced by wild type $V$. dahliae (Figure $3 A$; B). To confirm that the transgenes were expressed, expression of Ave1, ViAve1-1, ViAve1-5 and ViAve1-9 by V. dahliae 
in planta was analyzed. All genes were expressed at a similar level as Ave1 by $\mathrm{V}$. dahliae wild type (Figure 3D). Thus, ViAve1-1, ViAve1-5 and ViAve1-9 are unable to complement a $V$. dahliae $V d A v e 1$ deletion strain, suggesting that these homologs display (a) different function(s) than $\mathrm{V}$. dahliae VdAve1.
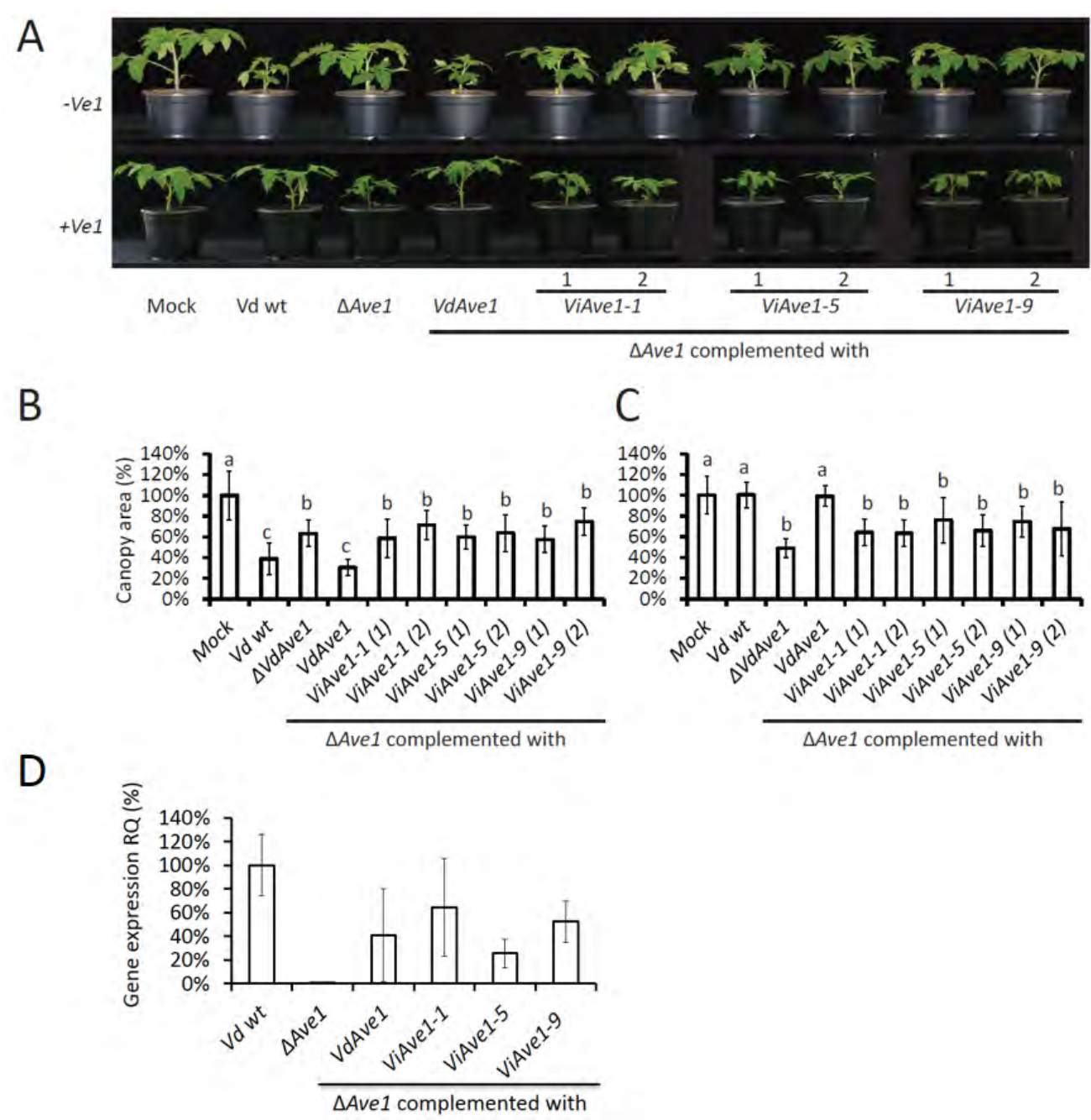

FIGURE 3 | Ave1 homologs derived from Venturia inaequalis cannot complement loss of Verticillium dahliae Ave1 (A) Complementation of V. dahliae $\triangle$ Ave1 with ViAve1-1, ViAve1-5 and ViAve1-9 on tomato plants lacking Ve1 (top) and Ve1 tomato (bottom) (B) Canopy area of tomato plants lacking Ve1 inoculated with V. dahliae $\triangle A v e 1$ expressing ViAve1-1, ViAve1-5 and ViAve1-9 at $14 \mathrm{dpi}(\mathrm{n}=8)$. (C) Canopy area of $V e 1$ tomato plants inoculated with $V$. dahliae $\triangle A v e 1$ expressing ViAve1-1, ViAve1-5 and ViAve1-9 at 14 dpi $(n=8)$. Data are presented as the mean \pm standard deviation. Different letters indicate significant differences ( $P<0.05$; One-Way ANOVA). (D) Gene expression of transgenes in a $V$. dahliae VdAve1 deletion strain when compared with VdAve1 expression in wild type $V$. dahliae. 


\section{Ave 1 homologs derived from Venturia inaequalis are unable to initiate an immune response in $\mathrm{Ve} 1$ tomato plants}

From the co-expression analysis on $\mathrm{N}$. benthamiana we concluded that the Venturia Ave1 homologs are not recognized by tomato Ve1 due to lack of a visible HR. Nevertheless, we have previously shown that $V e 1$ is able to establish immunity without signs of an HR under particular conditions (Zhang et al. 2013a). We therefore tested whether the $V$. dahliae transformants expressing ViAve1-1, ViAve1-5 and ViAve1-9 are contained when inoculated on tomato plants carrying Ve1 (Figure 3A; C). Tomato plants that were inoculated with wild type $V$. dahliae and the V. dahliae Ave1 deletion strain that was complemented with VdAve1 as a control showed no symptoms of Verticillium wilt, indicating that they are successfully contained upon recognition by Ve1. In contrast, the tomato plants inoculated with the Ave1 deletion strain and the transformants carrying expression constructs for ViAve1-1, ViAve1-5 and ViAve1-9 showed clear symptoms of Verticillium wilt. Together with the coexpression data this confirms that ViAve1-1, ViAve1-5 and ViAve1-9 are unable to induce a Ve1-mediated immune response.

\section{Discussion}

We have previously shown that the Verticillium dahliae effector VdAve 1 is recognized by the tomato immune receptor Ve1, and that homologs of Ave1 identified in particular other plant pathogens that share between 52 and $95 \%$ similarity are differentially recognized by Ve1 (de Jonge et al. 2012). Only one homolog was found in each of those plant pathogens, except for $V$. dahliae where subsequent analysis has shown that most isolates contain an additional Ave1-like (Ave1L) allele that shares between 64 and $68 \%$ sequence similarity with Ave1 (Chapter 6 of this thesis). In the genomes of the apple scab fungus $V$. inaequalis and the pear scab fungus $V$. pirinia largely expanded families of Ave 1 homologs were identified (Cooke et al. 2014). These homologs share between 51 and $63 \%$ sequence similarity with Ave1. Alignment of VdAve1 and the Venturia Ave1 homologs shows that there is no specific region of the protein that is highly conserved. In this study we showed that Ave 1 homologs derived from $V$. inaequalis and $V$. pirina are not recognized by $V e 1$. A reason for the lack of recognition might be the fact that $V$. inaequalis and $V$. pirina never co-evolved with $V e 1$ since tomato is not a host for these pathogens. Homologs of Ve1 have been found in many plant species, including functional homologs in various plants including tobacco, potato, eggplant and hop (Song et al. 2017a). Possibly, Ve1 homologs occur in apple and pear as well, that may potentially recognize one or more of the Ave 1 homologs derived from $V$. inaequalis and $V$. pirina, respectively. Possible recognition by an immune receptor can be tested by 
performing a transient expression assay with ViAve1-1, ViAve1-5 and ViAve1-9 on apple or pear (Li et al. 2004; Vleeshouwers and Oliver 2014).

We also showed that Ave1 homologs ViAve1-1, ViAve1-5 and ViAve1-9 derived from $V$. inaequalis that are highly expressed in planta cannot complement the virulence function of a $V$. dahliae Ave1 deletion strain on tomato. Possibly, to exert its function, Ave1 targets a host specific protein. If ViAve1-1, ViAve1-5 and ViAve1-9 have a similar function as Ave1 and target a host-specific protein, this protein may be absent from the Verticillium-tomato interaction and therefore Ave1 homologs cannot exert their function in other host plants. Host specificity of effectors has been described for the closely related oomycetes Phytophtora infestans and P. mirabilis that are pathogens on different hosts (Dong et al. 2014). Effector homologs derived from both pathogens are more efficient on their specific host target than on the foreign target. To test whether one or more of the Ave1 homologs are contributing to the virulence of $V$. inaequalis, individual deletion strains of ViAve1-1, ViAve1-5 and ViAve1-9 should be generated or gene silencing of multiple genes should be performed (Fitzgerald et al. 2004; Fitzgerald et al. 2003) and analyzed for full virulence on their host plant apple. However, unfortunately, targeted gene deletion is not straightforward in Venturia spp.

\section{Materials and methods}

\section{Plant material}

Tobacco (Nicotiana tabacum cv. Petite Havana SR1) and tomato (Solanum lycopersicum cv. MoneyMaker) plants were grown in the greenhouse at $21^{\circ} \mathrm{C} / 19^{\circ} \mathrm{C}$ during 16/8 hours day/night periods, respectively, with $70 \%$ relative humidity and $100 \mathrm{~W} / \mathrm{m}^{-2}$ supplemental light when the light intensity dropped below $150 \mathrm{~W} / \mathrm{m}^{-2}$.

\section{Generation of expression constructs}

The coding sequences (CDSs) of ViAve1-1, ViAve1-2, ViAve1-4, ViAve1-5, ViAve1-8, ViAve1-9, ViAve1-10 and VpAve1-1, VpAve1-2, VpAve1-3, VpAve1-4, VpAve1-5, VpAve1-6, VpAve1-7, VpAve1-11, were amplified from genomic DNA of Venturia inaequalis and $V$. pirina, respectively. The CDS was then cloned into pENTR-D (Invitrogen, Carlsbad, California) and subsequently transferred to the Gatewaycompatible destination vector pSol2092 (Zhang et al. 2013b) to generate expression constructs driven by the constitutive CaMV35S promoter for Agrobacterium tumefaciens-mediated transient expression. The CDSs of ViAve1-1, ViAve1-5 and ViAve1-9 were cloned into destination vector pFBT005 to generate expression constructs driven by the VdAve1 promoter for expression in V. dahliae. 


\section{Agrobacterium tumefaciens-mediated transient expression}

A. tumefaciens GV3101 strains containing the expression constructs in pSol2092 were grown for two nights in liquid Yeast Extract Broth (YEB) and then resuspended to an $\mathrm{OD}_{600}$ of 2 in infiltration medium as previously described (Zhang et al. 2013b). A. tumefaciens strains that carry the expression constructs for the $V$. dahliae and Venturia Ave1 homologs and tomato Ve1 were mixed in a 1:1 ratio and infiltrated in the lower side of 5- to 6 weeks-old tobacco leafs. At 5 dpi the leafs were inspected for the presence or absence of a hypersensitive response.

\section{Complementation of Verticillium dahliae Ave 1 deletion strains}

The A. tumefaciens Agl1 strain containing the expression construct for ViAve1-1, ViAve1-5 or ViAve1-9 in pFBT005, respectively, driven by the VdAve1 promoter, was used to transform a V. dahliae strain JR2 Ave1 deletion strain (de Jonge et al. 2012) as previously described (Santhanam 2012). Genomic DNA was extracted from transformants to verify the presence of ViAve1-1, ViAve1-5 or ViAve1-9 and the presence of the nourseothricin cassette.

\section{V. dahliae assay on tomato}

For $V$. dahliae inoculations 10-day-old tomato seedlings were uprooted, rinsed in water and then dipped for 5 minutes in a suspension of $10^{6}$ conidiospores per $\mathrm{mL}$ of water harvested from 1- to 2-week-old V. dahliae cultures on PDA as previously described (Fradin et al. 2009). Control plants received the same treatment, but the roots were dipped in water without conidiospores. After replanting in fresh soil, plants were incubated at standard greenhouse conditions. Disease development was monitored up to $21 \mathrm{dpi}$. The canopy area of 8 plants was measured with ImageJ software and a One-Way ANOVA was performed with IBM SPSS statistics software.

\section{Acknowledgements}

Work in the laboratory of BPHJT is supported by the Research Council Earth and Life Sciences (ALW) of the Netherlands Organization for Scientific Research (NWO). We thank Bert Essenstam and Pauline Sanderson of Unifarm for excellent plant care. 


\section{References}

Bowen, J. K., Mesarich, C. H., Bus, V. G., Beresford, R. M., Plummer, K. M., and Templeton, M. D. 2011. Venturia inaequalis: the causal agent of apple scab. Mol Plant Pathol 12:105-122.

Bus, V. G., Rikkerink, E. H., Caffier, V., Durel, C.-E., and Plummer, K. M. 2011. Revision of the nomenclature of the differential host-pathogen interactions of Venturia inaequalis and Malus. Annu Rev Phytopathol 49:391-413.

Cook, D. E., Mesarich, C. H., and Thomma, B. P. H. J. 2015. Understanding plant immunity as a surveillance system to detect invasion. Annu Rev Phytopathol.

Cooke, I. R., Jones, D., Bowen, J. K., Deng, C., Faou, P., Hall, N. E., Jayachandran, V., Liem, M., Taranto, A. P., Plummer, K. M., and Mathivanan, S. 2014. Proteogenomic analysis of the Venturia pirina (Pear Scab Fungus) secretome reveals potential effectors. J Proteome Res 13:3635-3644.

de Jonge, R., van Esse, H. P., Maruthachalam, K., Bolton, M. D., Santhanam, P., Saber, M. K., Zhang, Z., Usami, T., Lievens, B., Subbarao, K. V., and Thomma, B. P. H. J. 2012. Tomato immune receptor Ve1 recognizes effector of multiple fungal pathogens uncovered by genome and RNA sequencing. Proc Natl Acad Sci U S A 109:5110-5115.

Dong, S., Stam, R., Cano, L. M., Song, J., Sklenar, J., Yoshida, K., Bozkurt, T. O., Oliva, R., Liu, Z., Tian, M., Win, J., Banfield, M. J., Jones, A. M., van der Hoorn, R. A., and Kamoun, S. 2014. Effector specialization in a lineage of the Irish potato famine pathogen. Science 343:552-555.

Fitzgerald, A., van Kan, J. A., and Plummer, K. M. 2004. Simultaneous silencing of multiple genes in the apple scab fungus, Venturia inaequalis, by expression of RNA with chimeric inverted repeats. Fungal Genet Biol 41:963-971.

Fitzgerald, A. M., Mudge, A. M., Gleave, A. P., and Plummer, K. M. 2003. Agrobacterium and PEGmediated transformation of the phytopathogen Venturia inaequalis. Mycological research 107:803810.

Fradin, E. F., and Thomma, B. P. H. J. 2006. Physiology and molecular aspects of Verticillium wilt diseases caused by V. dahliae and V. albo-atrum. Mol Plant Pathol 7:71-86.

Fradin, E. F., Zhang, Z., Juarez Ayala, J. C., Castroverde, C. D., Nazar, R. N., Robb, J., Liu, C. M., and Thomma, B. P. H. J. 2009. Genetic dissection of Verticillium wilt resistance mediated by tomato Ve1. Plant Physiol 150:320-332.

Fudal, I., Ross, S., Gout, L., Blaise, F., Kuhn, M., Eckert, M., Cattolico, L., Bernard-Samain, S., Balesdent, M., and Rouxel, T. 2007. Heterochromatin-like regions as ecological niches for avirulence genes in the Leptosphaeria maculans genome: map-based cloning of AvrLm6. Mol. Plant Microbe Interact. 20:459-470.

Gessler, C., Patocchi, A., Sansavini, S., Tartarini, S., and Gianfranceschi, L. 2006. Venturia inaequalis resistance in apple. CRC Crit Rev Plant Sci 25:473-503.

Grandaubert, J., Lowe, R. G., Soyer, J. L., Schoch, C. L., Van de Wouw, A. P., Fudal, I., Robbertse, B., Lapalu, N., Links, M. G., and Ollivier, B. 2014. Transposable element-assisted evolution and adaptation to host plant within the Leptosphaeria maculans-Leptosphaeria biglobosa species complex of fungal pathogens. BMC Genomics 15:1.

Jones, J. D., and Dangl, J. L. 2006. The plant immune system. Nature 444:323-329.

Kucheryava, N., Bowen, J. K., Sutherland, P. W., Conolly, J. J., Mesarich, C. H., Rikkerink, E. H., Kemen, E., Plummer, K. M., Hahn, M., and Templeton, M. D. 2008. Two novel Venturia inaequalis genes induced upon morphogenetic differentiation during infection and in vitro growth on cellophane. Fungal Genet Biol 45:1329-1339.

Li, C., Sasaki, N., Isogai, M., and Yoshikawa, N. 2004. Stable expression of foreign proteins in herbaceous and apple plants using Apple latent spherical virus RNA2 vectors. Archives of virology 149:15411558.

Rovenich, H., Boshoven, J. C., and Thomma, B. P. H. J. 2014. Filamentous pathogen effector functions: of pathogens, hosts and microbiomes. Curr Opin Plant Biol 20:96-103. 
Santhanam, P. 2012. Random insertional mutagenesis in fungal genomes to identify virulence factors. Pages 509-517 in: Plant fungal pathogens. Methods in molecular biology, vol. 835. T. B. Bolton MD, ed. Humana press, Totowa.

Shabi, E., Rotem, J., and Loebenstein, G. 1973. Physiological races of Venturia pirina on pear. Phytopathology 63:41-43.

Shiller, J., Van de Wouw, A. P., Taranto, A. P., Bowen, J. K., Dubois, D., Robinson, A., Deng, C. H., and Plummer, K. M. 2015. A Large Family of AvrLm6-like Genes in the Apple and Pear Scab Pathogens, Venturia inaequalis and Venturia pirina. Front Plant Sci 6:980.

Song, Y., Zhang, Z., Seidl, M. F., Majer, A., Jakse, J., Javornik, B., and Thomma, B. P. 2017a. Broad taxonomic characterization of Verticillium wilt resistance genes reveals ancient origin of the tomato Ve1 immune receptor. Mol Plant Pathol 18:195-209.

Thomma, B. P. H. J., Nurnberger, T., and Joosten, M. H. A. J. 2011. Of PAMPs and effectors: The blurred PTI-ETI dichotomy. Plant Cell 23:4-15.

Vleeshouwers, V. G., and Oliver, R. P. 2014. Effectors as tools in disease resistance breeding against biotrophic, hemibiotrophic, and necrotrophic plant pathogens. Mol. Plant Microbe Interact. 27:196-206.

Zhang, Z., Esse, H. P., Damme, M., Fradin, E. F., Liu, C. M., and Thomma, B. P. H. J. 2013a. Ve1-mediated resistance against Verticillium does not involve a hypersensitive response in Arabidopsis. Mol Plant Pathol 14:719-727.

Zhang, Z., Fradin, E., de Jonge, R., van Esse, H. P., Smit, P., Liu, C.-M., and Thomma, B. P. H. J. 2013 b. Optimized agroinfiltration and virus-induced gene silencing to study Ve1-mediated Verticillium resistance in tobacco. Mol. Plant Microbe Interact. 26:182-190. 


\section{An Ave 1-like effector displays allelic variation within the Verticillium dahliae population}

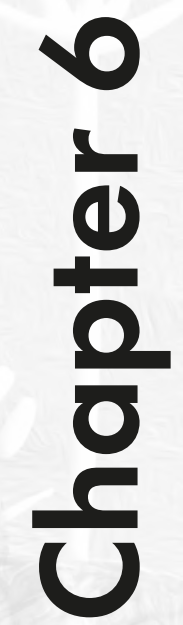

Jordi C. Boshoven

Hanna Rovenich*

Yin Song*

Luigi Faino

Michael F Seid" ${ }^{\#}$

Bart PHJ Thomma\#

\#These authors contributed equally

"These authors contributed equally 
Verticillium dahliae is a soil-borne fungal pathogen that causes vascular wilt disease on a broad range of host plants. The tomato immune receptor $V e 1$ is able to recognize race 1 strains of $V$. dahliae that secrete the Ave1 effector. We identified a gene in V. dahliae strain JR2 with high similarity to the Ave1 effector gene that we called Ave1L (for Ave1like). Subsequently, we identified in total five alleles (Ave1L1 to Ave1L5) in the $V$. dahliae population. While some of the $V$. dahliae strains do not have Ave1L, other strains have one of the five Ave $1 L$ alleles in presence or absence of Ave1. The Ave1 $\mathrm{L}$ alleles contain multiple single nucleotide polymorphisms that result in a stop codon in some of the alleles. In addition, one of the alleles contains a retrotransposon. These observations suggest that Ave1L experienced significant selection pressure, potentially to evade recognition by an immune receptor. Thus, we attempted to predict the common progenitor sequence of the five Ave $1 \mathrm{~L}$ alleles. However, we show that neither of the Ave $1 \mathrm{~L}$ alleles, nor the progenitor, are recognized by the tomato immune receptor Ve2 that shows high homology to Ve1. We furthermore show that Ave1L2 is expressed during host colonization but does not seem to contribute to virulence on tomato. Since $V$. dahliae has a wide host range, Ave1L potentially contributes to virulence on other host plants than tomato where it may have been recognized by an immune receptor that drove its divergence. 


\section{Introduction}

Plants can sense invading microbial pathogens through the recognition of conserved microbe-associated molecular patterns (MAMPs) such as bacterial flagellin, fungal chitin and oomycete $\beta$-glucans (Dodds and Rathjen 2010; Thomma et al. 2011). Recognition of MAMPs leads to the activation of immune responses to stop pathogen ingress. To gain further access to the host, pathogens produce effectors; secreted molecules that support host colonization. Although effectors may contribute to host colonization in many ways, most effectors that have been functionally characterized up to now specifically target host immune responses (Rovenich et al. 2014). In response to pathogen exploitation of effectors, plants evolved immune receptors that can monitor (the activity of) specific effectors to re-initiate immune responses, typically referred to as effector-triggered immunity (ETI) (Dodds and Rathjen 2010; Thomma et al. 2011). To overcome effector recognition, pathogens in turn may lose the recognized effector, evolve mutants that are no longer recognized or, alternatively, evolve novel effectors that can suppress the immune response. As a result of all this, plant immunity evolved to a robust system to detect invasion in which immunogenic molecules form a continuum and can be host- or microbederived and can have various intrinsic functions. Ultimately host receptors, termed invasion pattern receptors (IPRs), detect externally encoded as well as modified-self ligands, termed invasion patterns, that betray microbial invasion (Cook et al. 2015; Thomma et al. 2011).

The Cladosporium fulvum-tomato interaction is a well-established fungal model system that has been used to study effector-receptor interactions (de Wit 2016; Thomma et al. 2005). The first fungal avirulence gene was cloned from C. fulvum and to date a dozen additional effector genes have been cloned most of which act as avirulence genes (Bolton et al. 2008; Joosten et al. 1994; Lauge et al. 2000; Luderer et al. 2002; Mesarich et al. 2014; Stergiopoulos et al. 2007; Van den Ackerveken et al. 1993; van Kan et al. 1991; Westerink et al. 2004). As a consequence, variation within a fungal effector was also first reported in C. fulvum for which isoforms of the Avr4 effector were identified that are no longer recognized by the corresponding tomato immune receptor $\mathrm{Cf}-4$ as a result of a frameshift mutation or a single point mutation in the Avr4 gene that induces a single amino acid change (Joosten et al. 1997; Joosten et al. 1994). Other alterations were observed in C. fulvum effector Avr2 where, besides a frameshift mutation, the introduction of a premature stop codon or a retrotransposon in the gene results in a truncated protein that is no longer recognized by the corresponding tomato immune receptor Cf-2 (Luderer et al. 2002). In the case of C. fulvum effectors Avr4E and Avr9 some alleles contain point mutations while in other strains the complete gene is 
deleted to avoid recognition by the corresponding tomato immune receptor Cf4E and Cf-9, respectively (van Kan et al. 1991; Westerink et al. 2004). Importantly, while effector deletion or mutation can overcome effector recognition in plants carrying the corresponding immune receptor, such modification may compromise virulence on plants that lack this immune receptor. Although not yet observed in C. fulvum, pathogens may also evolve novel effectors to suppress recognition of a particular effector. This has been demonstrated for Avr1 of the tomato pathogen Fusarium oxysporum $\mathrm{f}$. sp. lycopersici that can suppress immune responses that are activated by the tomato immune receptors I-2 and I-3 that recognize the Avr2 and Avr3 effectors, respectively (Houterman et al. 2008). Collectively, these examples illustrate that pathogens have evolved many ways to circumvent recognition by immune receptors of their host plants.

Verticillium dahliae is a soil-borne vascular pathogen and causal agent of vascular wilt disease in a broad range of plant species (Fradin and Thomma 2006). In contrast to the situation for $C$. fulvum for which many complex races have been described on tomato, the situation for $V$. dahliae is simple on that same host due to the existence of only a single dominant resistance source that divides the population in only two races. On tomato, race 1 strains of $V$. dahliae are contained by the Ve 1 immune receptor whereas race 2 strains are not recognized (Fradin et al. 2009; Kawchuk et al. 2001). Recently, the race 1-specific effector that is recognized by Ve1 was identified as Ave1 (for Avirulence on Ve1 tomato) (de Jonge et al. 2012). Interestingly, it was found that all race 2 strains that were analysed lack the complete Ave1 gene. Moreover, it was shown that Ave1 contributes to the virulence of $V$. dahliae on plants that lack Ve1. Intriguingly, the Ave1 CDS is completely identical in all 85 race 1 strains that have been analyzed to date, suggesting that sequence conservation is important for virulence (de Jonge et al. 2012). Intriguingly, many homologs of Ave 1 are found in plants as well as in a few plant pathogens (de Jonge et al. 2012). Most of the plant homologs are annotated as plant natriuretic peptides (PNPs) or expansin-like proteins that are thought to be involved in water and ion homeostasis and therefore in many fundamental plant processes including growth, net water uptake, photosynthesis, stomatal opening and gas exchange (Gehring and Irving 2003; Ludidi et al. 2004; Maryani et al. 2001; Pharmawati et al. 2001). Interestingly, the Ave 1 homolog of the bacterial pathogen $X$. axonopodis, known as XacPNP, acts as a virulence factor by affecting homeostasis and photosynthesis in citrus to stimulate bacterial proliferation (Garavaglia et al. 2010; Gottig et al. 2008). The Ave 1 homologs that occur in plant pathogens do not follow the phylogeny of the species in which they occur, which is interpreted as a sign of horizontal gene transfer, leading to believe that the microbial Ave 1 homologs have been acquired from plants (de Jonge et al. 2012; Gottig et al. 2008) 
In this study, a BLAST search using Ave1 against the recently finished gapless genome of $V$. dahliae strain JR2 (Faino et al. 2015) revealed an Ave1-like gene. Subsequent BLAST searches identified allelic variation in a population of $\mathrm{V}$. dahliae strains.

\section{Results}

\section{Identification of an Ave 1-like gene in Verticillium dahliae}

Using the genome of V. dahliae strain JR2 (Faino et al. 2015), a BLAST analysis using Ave 1 as a query identified a gene with $67 \%$ similarity that we will further refer to as Ave1L (for Ave1-like). Intriguingly, while Ave1 encodes a protein of 134 amino acids (de Jonge et al. 2012), the CDS of Ave1L likely encodes a protein of only 24 amino acids due to a predicted stop codon. To determine whether Ave1L can be found in other $V$. dahliae strains as well, we performed BLAST analyses using Ave $1 \mathrm{~L}$ as a query on twenty recently sequenced $V$. dahliae genomes (Table 1) (de Jonge et al. 2012; Faino et al. 2015; Klosterman et al. 2011). While no Ave1L sequence was found in four genomes, the genomes of $16 \mathrm{~V}$. dahliae strains were each found to carry a single Ave $1 \mathrm{~L}$ copy that displays $93-100 \%$ similarity to the initially identified Ave1L, while displaying 65-68\% similarity to Ave1. Collectively, five different Ave1L alleles were identified, designated Ave1L1 to Ave1L5. Following the phylogeny of the V. dahliae strains, Ave1L1 was found in five strains that include JR2 and VdLs17 (de Jonge et al. 2012; Faino et al. 2015; Klosterman et al. 2011), Ave1L2 in three strains, Ave1L3 in five strains, Ave1L4 in one strain and Ave1L5 in two strains (Table 1, Figure 1B). Similar to the initially identified Ave $1 L 1$, also Ave $1 L 3$ and Ave $1 L 4$ are predicted to encode proteins of 24 amino acids. In contrast, Ave1L2 and Ave1L5 are predicted to encode proteins of 134 amino acids similar to Ave1 (Figure 1A). Interestingly, the presence of Ave $1 \mathrm{~L}$ alleles did not correlate with presence of Ave1, as Ave $1 \mathrm{~L}$ alleles were found in race 1 as well as in race 2 strains (Table 1, Figure 1B).

Like Ave1, Ave1L shows similarity to plant natriuretic peptides (PNPs) or expansinlike proteins (de Jonge et al. 2012). To analyse whether Ave1L carries a conserved PNP domain as has been characterized in Arabidopsis thaliana PNP (AtPNP-A) (Ludidi et al. 2004; Morse et al. 2004) the amino acid sequences of Ave1, Ave1L and various PNPs were aligned to AtPNP-A and similarity was determined (Fig. 1C). Intriguingly, there does not seem to be a particularly conserved region among the proteins, and also the region that aligns with the PNP domain of AtPNP-A is not particularly conserved (Fig. 1C). The bacterial pathogen $X$. axonopodis secretes a PNP-like effector known as XacPNP that modulates host responses to facilitate bacterial proliferation on citrus. XacPNP comprises a 12 amino acid peptide that 
displays a high degree of similarity to the PNP domain of AtPNP-A, and that exerts the biological activity of XacPNP (Gottig et al. 2008). However, Ave1 L does not carry this 12 amino acid peptide, in contrast to Ave1 and the other PNPs included in the alignment (Figure 1C) (Gottig et al. 2008). This finding suggests that Ave1 L does not function as a natriuretic peptide.

TABLE 1 | Ave1L alleles identified in sequenced V. dahliae genomes

\begin{tabular}{|c|c|c|c|c|c|c|}
\hline Strain & Origin & Isolated from & Race** & Ave1 & $\begin{array}{l}\text { Ave1L } \\
\text { allele }\end{array}$ & $\begin{array}{l}\text { Number and } \\
\text { position of } \\
\text { premature stop } \\
\text { codon(s) }\end{array}$ \\
\hline$J_{R} 2^{\$}$ & Canada & Solanum lycopersicum & 1 & Yes & \multirow{5}{*}{ Ave1L1 } & \multirow{5}{*}{$1(25 W)$} \\
\hline $\operatorname{VdLs} 17^{\Delta}$ & USA & Lactuca sativa & 2 & No & & \\
\hline St16.01* & Syria & Gossypium spp & 2 & No & & \\
\hline $\operatorname{Vd} 57^{*}$ & Germany & Fragaria $\mathrm{x}$ ananassa & 2 & No & & \\
\hline $\mathrm{V}^{* *}$ & Netherlands & Chrysanthemum spp. & 2 & No & & \\
\hline CBS38166\$ & Canada & Solanum lycopersicum & 1 & Yes & \multirow{3}{*}{ Ave1L2 } & \multirow{3}{*}{ No } \\
\hline St14.01\$ & USA & Pistacia vera & 1 & Yes & & \\
\hline DVD-S26\$ & Canada & Soil & 2 & No & & \\
\hline DVD-3\$ & Canada & Solanum tuberosum & 2 & No & \multirow{5}{*}{ Ave1L3 } & \multirow{5}{*}{$\begin{array}{l}4(25 W, 57 Q \\
1200,1280)\end{array}$} \\
\hline DVD-31\$ & Canada & Solanum lycopersicum & 2 & No & & \\
\hline DVD-S94\$ & Canada & Soil & 2 & No & & \\
\hline DVD-161\$ & Canada & Solanum tuberosum & 2 & No & & \\
\hline $\mathrm{JKG} 8^{\#^{\star}}$ & Netherlands & Solanum tuberosum & 2 & No & & \\
\hline DVD-S29\$ & Canada & Soil & 2 & No & Ave1L4 & $1(25 W)$ \\
\hline St. $100^{\mu}$ & Belgium & Soil & 2 & No & \multirow{2}{*}{ Ave1L5 } & \multirow{2}{*}{ No } \\
\hline $463^{*}$ & Mexico & Gossypium spp. & 2 & No & & \\
\hline $2009-605^{\star}$ & Ukraine & Capsicum annuum & 1 & Yes & \multirow{4}{*}{ No } & \multirow{4}{*}{$x$} \\
\hline Vd152* & Hungary & Quercus spp & 2 & No & & \\
\hline $\operatorname{Vd} 52^{*}$ & Austria & Capsicum annuum & 1 & Yes & & \\
\hline Vd39* & Germany & Helianthus annuus & 2 & No & & \\
\hline
\end{tabular}

* newly sequenced $V$. dahliae genomes

** The race nomination in $V$. dahliae is restricted to strains that are pathogenic on Ve1 tomato in which race 1 strains are contained and race 2 strains are not.

\$ de Jonge et al., 2012

$\triangle$ Klosterman et al., 2011

\# Ispahani et al., 2008

$\mu$ de Jonge et al., 2013 
A

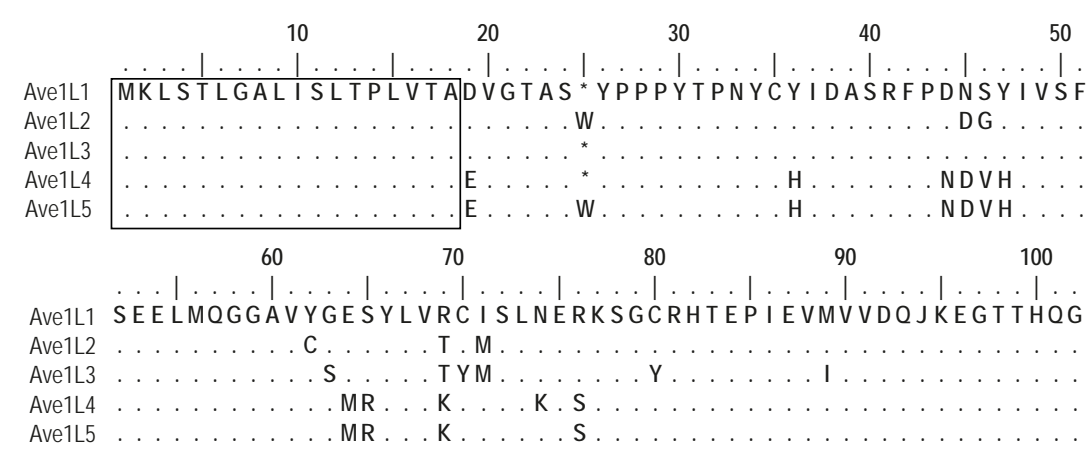

\begin{tabular}{|c|c|c|}
\hline & 120 & 130 \\
\hline ell & IVLSKEAY I ALVKQY & HSFIOQVEYR \\
\hline 112 & $\ldots \ldots \ldots$ & \\
\hline 113 & $.{ }^{*} . v$ & \\
\hline 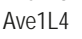 & $\ldots \ldots \ldots 1 \ldots$ & I. \\
\hline Avell 5 & $\mathrm{~K}$. & \\
\hline
\end{tabular}

B

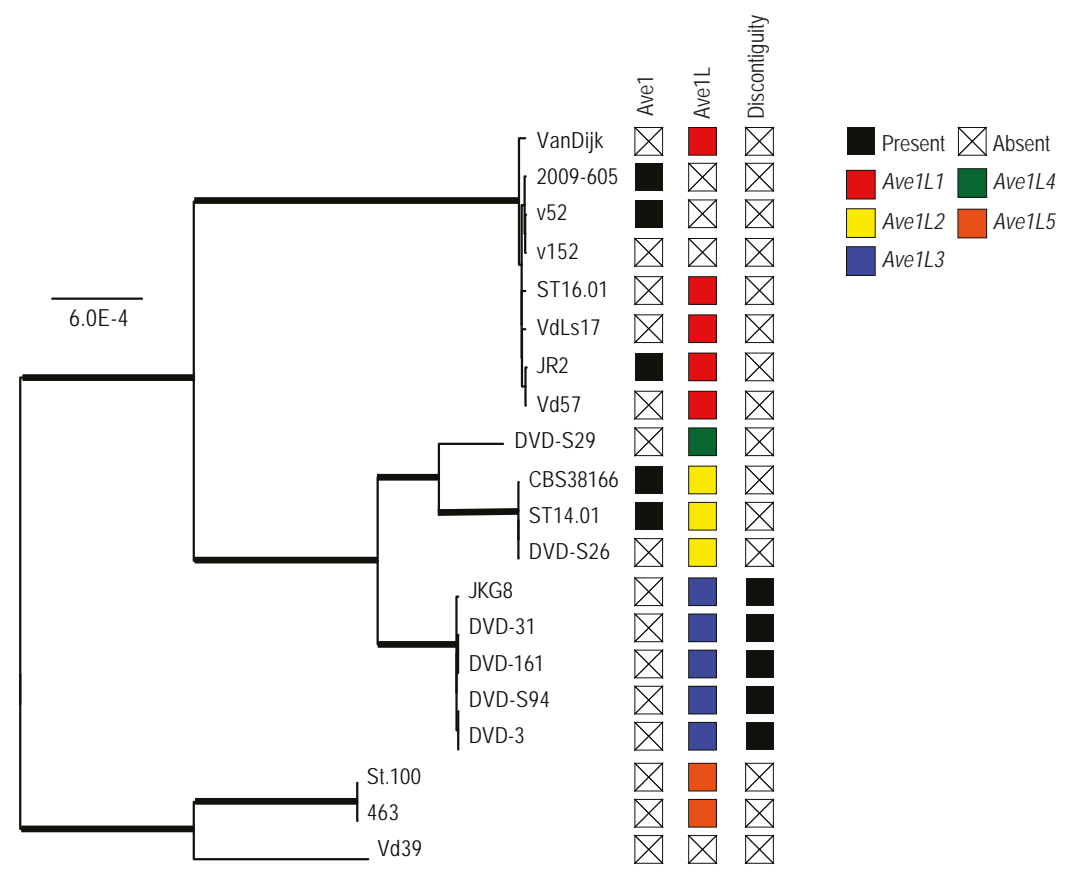

FIGURE 1 | The identification of five Ave1L alleles. (A) Alignment of predicted Ave1L proteins Ave1 L1 to Ave1L5. The black box indicates the predicted signal peptide, while asterisks $\left(^{\star}\right)$ indicate predicted stop codons and the hashtag (\#) indicates discontiguity. (B) Phylogenetic tree based on the genomes of $V$. dahliae strains and the presence of Ave1 and Ave $1 \mathrm{~L}$ alleles in the respective genomes. (C) continues at next page. 
C

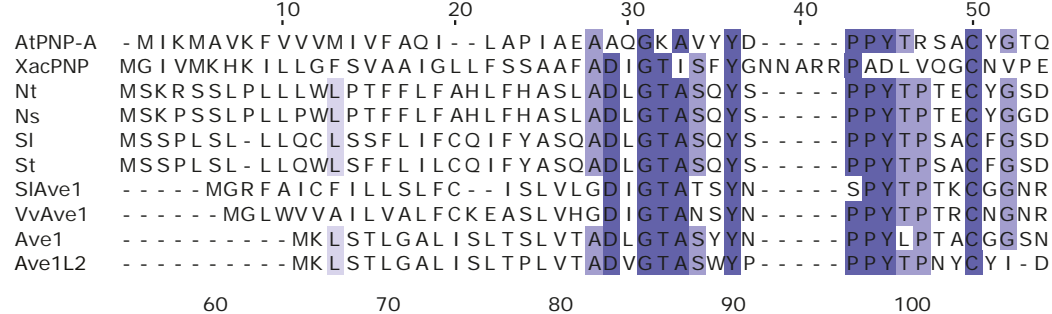

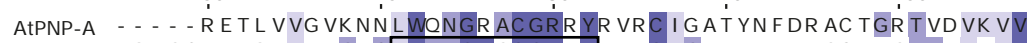
XaCPNP DQVSGRNYQVVTVSDGLWDNGASCGRRYRMRCISTPVKH - SCTASTIDVIVV Nt ATQFPSSNFFAAAGEGIWDNGAACGRQYL I SCINSVLPRA-CKHGETIQIKI NS ATQFPSSNFFAAAGEGI WDNGAACGRQYLISCINSVLPRA-CKHGETIQIK I SI STQFPSSNFFAAASEGIWDDGAACGRQYLISC I SSVLPKA-CKPGETIQIKI I St STQFPSSNFFAAASEG I WDDGAACGRQYLISCISSVLPKA-CKPGETIQIKIV SIAve1 SDQFPSGNLFVAVSEGLWDNGAACGRR YRLRCLSGNNRP - - CKEG - TIDVRVV VVAve1 QDQFPPGNLFVSVSEGLWDNGAACGRR YRLRCLSGAKKP - - CKDG - TVDVKV Avel PSQFPSGNLFVAVSDGLWDNGAACGRRYR I KCLSGARGS- - CKDG- MIDVRV Ave1L2 ASRFPDDGY I VSFSEELMQGGAVCGESYLVTCMSLNERKSGCRHTEPIEVMVV

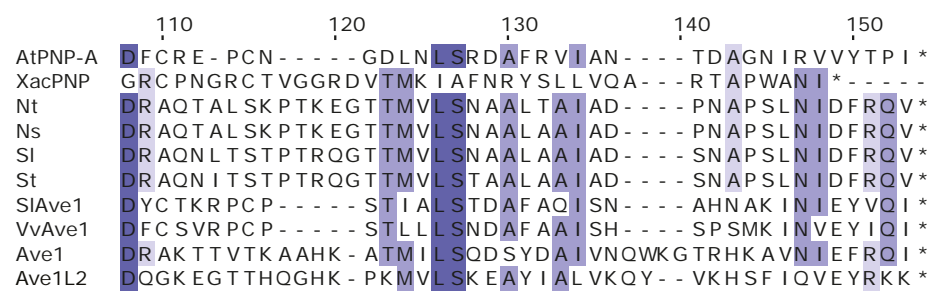

FIGURE 1 | The identification of five Ave1L alleles. (C) Alignment of AtPNP-A, XacPNP, PNPs (Nicotiana tomentosiformis Nt; N. sylvestris Ns; Solanum lycopersicum SI; S. tuberosum St; Vitis vinifera $\mathrm{Vv}$ ), Ave1 and Ave1L. The purple colour indicates amino acid conservation. The black box indicates the active PNP domain of XacPNP (Gottig et al. 2008).

\section{Ave $1 \mathrm{~L}$ alleles occur in a highly dynamic, lineage-specific, genomic region}

It was previously shown that extensive chromosomal rearrangements occur among strains of V. dahliae (de Jonge et al. 2013). The occurrence of these rearrangements coincides with the occurrence of lineage-specific (LS) regions that are only present in sub-sets of $V$. dahliae strains and that are significantly enriched for in plantaexpressed genes that also include effectors (de Jonge et al. 2013). Consequently, it has been suggested that chromosomal rearrangements facilitate adaptive evolution in $\mathrm{V}$. dahliae, giving rise to lineage-specific segmental duplications that may evolve new traits (de Jonge et al. 2013; Faino et al. 2015; Seidl and Thomma 2014). Since Ave1 $L$ does not occur in all $V$. dahliae strains, the gene likely occurs in an LS region. To confirm this, we aligned reads from the sixteen Ave $1 L$-carrying $V$. dahliae strains to the gapless genome of $V$. dahliae strain JR2 (Faino et al. 2015). We indeed found that the five Ave1 $\mathrm{L}$ alleles are positioned in the same genomic environment in a repeat-rich $L S$ region (Figure $2 A$ ). 


\section{Ave 1 L3 is disrupted by a transposable element}

We noticed that each Ave1L3 allele was consistently located on two contigs, which suggests discontiguity of this particular allele (Figure 2B). To confirm this in silico observation, we designed PCR primers on conserved sequences that are present in all Ave1 $L$ alleles, spanning the predicted site where the discontiguity occurs, as well as PCR primers that amplify a conserved region up- and downstream of this site. We used these primers on genomic DNA of a panel of $V$. dahliae strains that represent the five different Ave $1 L$ alleles. The primers spanning the site of the observed discontiguity in Ave1L3 amplified a $200 \mathrm{bp} \mathrm{PCR} \mathrm{product} \mathrm{from} \mathrm{strains}$ VdLs17 (Ave1L1), Dvd-S26 (Ave1L2), Dvd-S29 (Ave1L4) and St.100 (Ave1L5), while no PCR product was amplified from the Ave1L3-carrying strains DVD-3, DVD-31, DVD-S94, DVD-161. However, primers that were targeted to a region upstream and downstream of the predicted discontiguity amplified a product from all strains, including those carrying Ave1L3 (Figure 2C). These results confirm that Ave $1 \mathrm{~L} 3$ is discontiguous in the genome of all strains that carry this allele.

Discontiguity of Ave1L3 may be caused by the insertion of a transposable element or by a gross chromosomal rearrangement that occurred within the allele. To test whether Ave $1 L 3$ is disrupted by an insertion we repeated the PCR with primers spanning the site of the discontiguity using a significantly enhanced elongation time to allow for amplification of considerably larger PCR fragments. We used an Ave1L1-carrying $V$. dahliae strain as a control for absence of discontiguity. A fragment of around $7 \mathrm{~kb}$ was amplified from $V$. dahliae strain DVD-3 that carries Ave1L3 (Figure 2D). To determine the sequence of the PCR fragment we used an Oxford Nanopore MinION sequencer. Subsequently, we obtained a consensus sequence from the reads and performed a BLAST analysis. This analysis revealed that Ave $1 L 3$ is interrupted by a long terminal repeat retrotransposon that is classified as VdLTRE3 (Faino et al. 2016). 
A

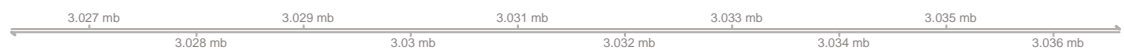

Avell 1

Avell2

Avell 3

Avell 4

Avell 5

no Avell
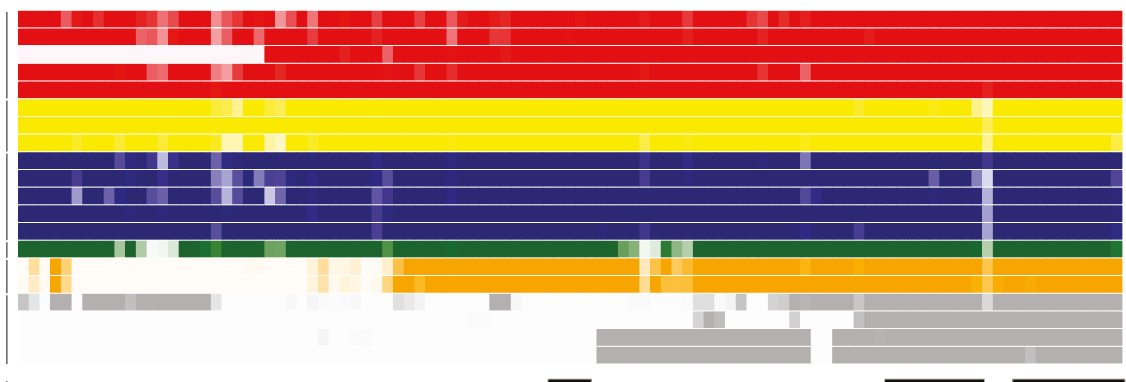

Genes

Repeats

B

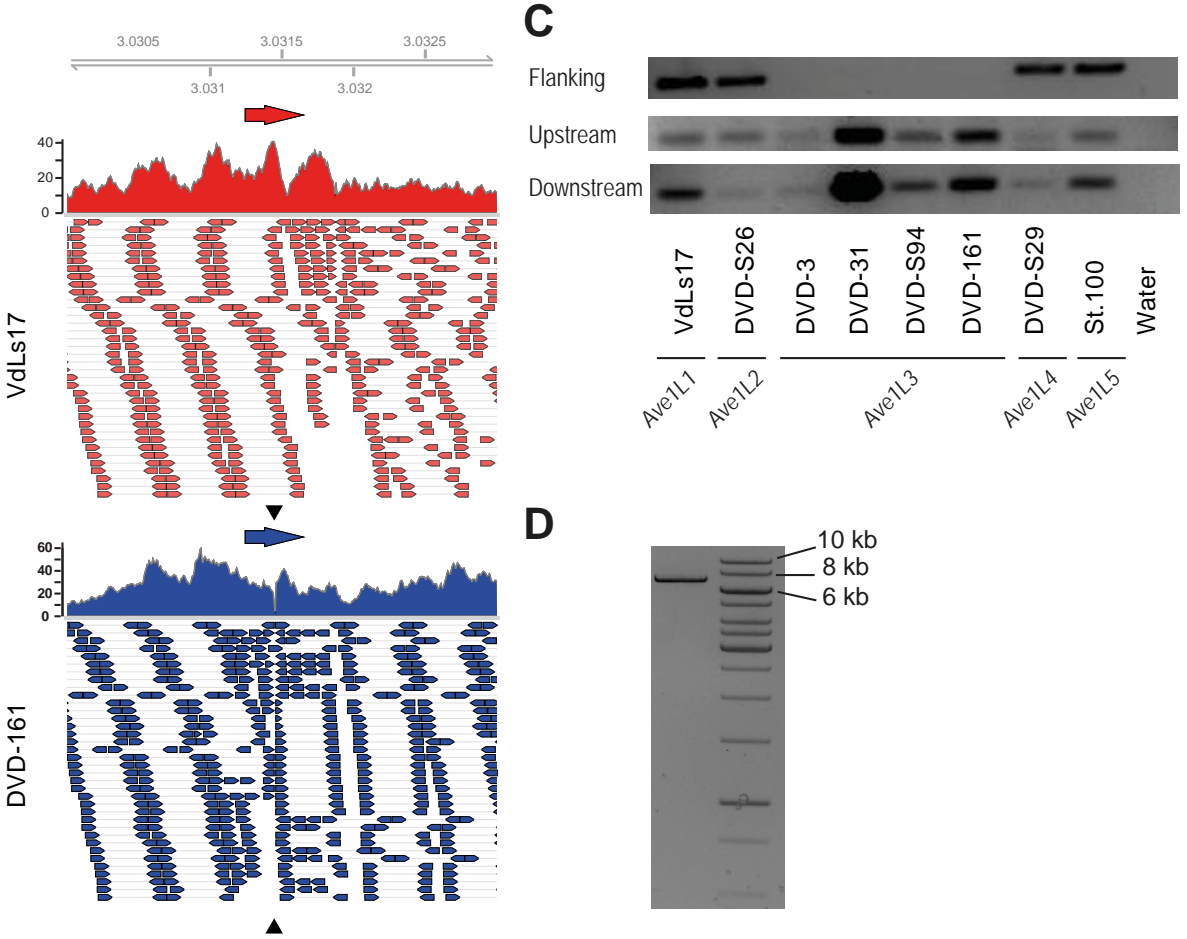

FIGURE 2 | The genomic context of the Ave1L alleles. (A) Each coloured bar represents the genomic context of $A v e 1 L$ in different $V$. dahliae strains. The red arrow indicates the position of the Ave1L gene while the black bars indicate other genes and repeats in the region. (B) Genomic region flanking $A v e 1 L$ and the read coverage in $V$. dahliae strain VdLs17 and DVD-161. The horizontal red and blue arrows indicate the position of Ave1L. The black arrows indicate discontiguity of Ave1L3 in DVD-161. (C) Amplification products on gel using primers spanning, are upstream, and downstream of $(*)$ the predicted side. The absence of an amplification product on gel when primers were used spanning the predicted side where discontiguity occurs confirms discontiguity in V. dahliae strains that carry Ave1L3. (D) The presence of an amplification product (left lane) confirms that Ave1 L3 contains an insert of approximately $7 \mathrm{~kb}$ deduced from the $10 \mathrm{~kb}$ ladder that was run alongside (right lane). 


\section{Ave 1 L2 is expressed during host colonization}

To assay whether any of the Ave $1 L$ alleles is expressed during host colonization, we inoculated Arabidopsis plants with V. dahliae strains VdLs17 (Ave1L1), DVD-S26 (Ave1L2), DVD-S94 (Ave1L3), DVD-S29 (Ave1L4) and St.100 (Ave1L5) and expression of the Ave1L alleles was monitored with real-time PCR at 14 and 21 days post inoculation, showing that only Ave1L2 is expressed in planta (Figure 3). To further confirm this observation, we monitored the expression of Ave1L2 in another strain as well. To this end, we used the race 1 strain St14.01 that carries Ave 1 L2 in addition to Ave1, in contrast to the race 2 strain DVD-S26 that carries Ave1L2 in absence of Ave1. Interestingly, also in this strain Ave1L2 was found to be expressed (Figure 3).

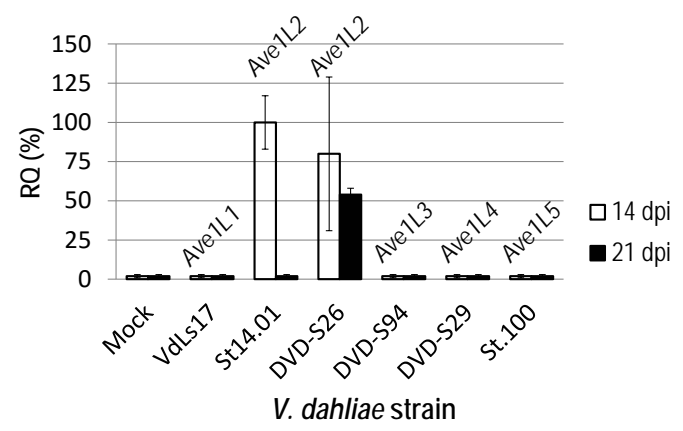

FIGURE 3 | Ave1L2 is expressed by V. dahliae on Arabidopsis. Ave1L expression in $V$. dahliae on Arabidopsis at 14 and $21 \mathrm{dpi}$ after mock treatment or inoculation with $V$. dahliae strain VdLs17, St14.01 DVD-S26, DVD-S94, DVD-S29 or St. 100.

\section{Ave 1 L2 is not a virulence factor on tomato plants}

Since Ave1L2 is expressed in planta we tested whether Ave1L2 is a virulence factor of $V$. dahliae. To this end, we deleted Ave1L2 from the genomes of $V$. dahliae strains St14.01 and CBS38166 (Supplemental Figure 1). We then inoculated three transformants of St14.01 and four transformants of CBS38166 on tomato plants and tested their aggressiveness in comparison to the corresponding wild type $\mathrm{V}$. dahliae strain. However, tomato plants inoculated with the Ave1L2 deletion strains showed a similar level of stunting as the corresponding wild type strains (Figure $4 \mathrm{~A} ; \mathrm{B})$. Moreover, a similar amount of fungal biomass was detected in tomato upon inoculation with the Ave1 L2 deletion strains as well as with the corresponding wild type strains (Figure 4C). Therefore, we conclude that Ave1L2 does not contribute to the virulence of $V$. dahliae on tomato. 
A
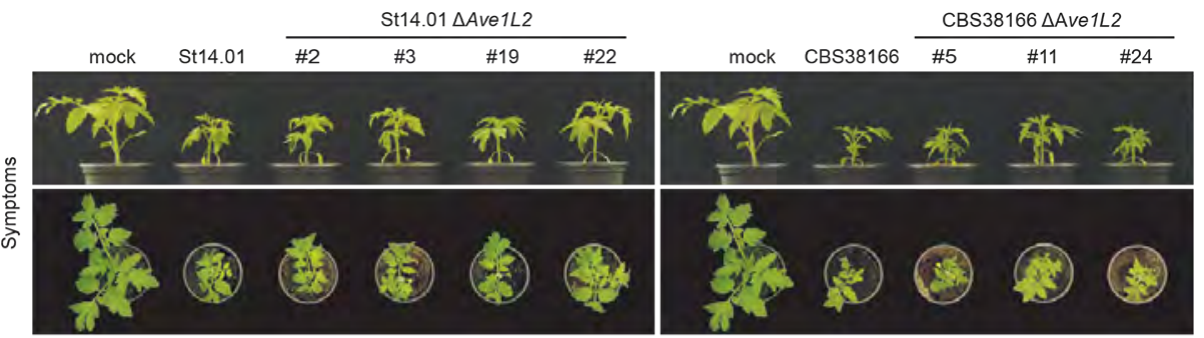

B
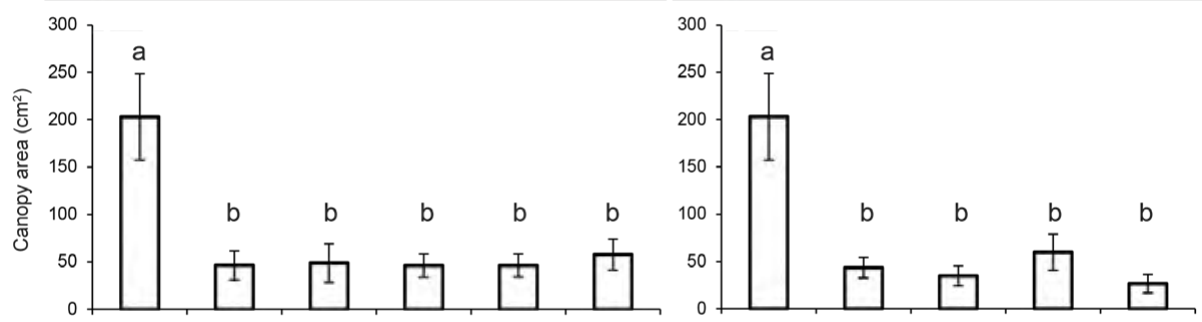

C
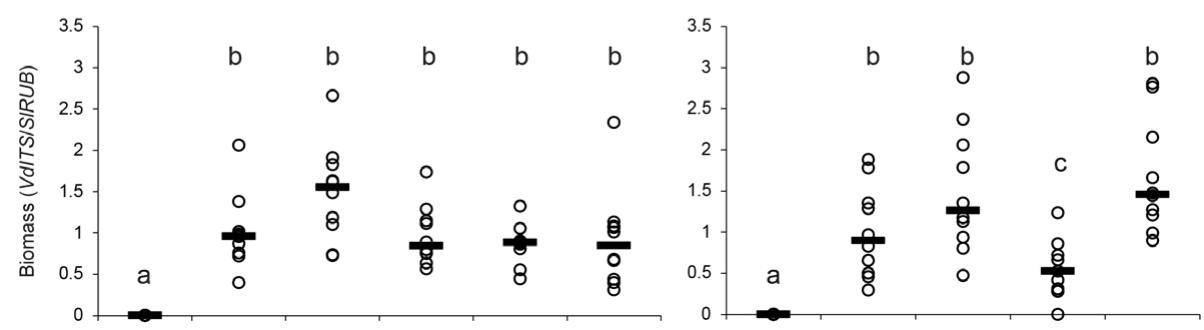

FIGURE 4 | Ave1L2 does not markedly contribute to virulence on tomato. (A) Tomato plants inoculated with Ave1L2 deletion strains (\#) and the corresponding wild strain (St14.01 or CBS38166). (B) The average canopy area of inoculated tomato plants at $14 \mathrm{dpi}(n=10)$. Error bars represent standard errors. (C) Data points indicate the accumulated fungal biomass in tomato plants at $14 \mathrm{dpi}$. The horizontal line indicates the average fungal biomass.

\section{Ave $1 \mathrm{~L}$ proteins are not recognized by $\mathrm{Ve} 2$}

Ve1 is the only plant immune receptor known to confer resistance to $V$. dahliae (Fradin et al. 2009; Kawchuk et al. 2001). In tomato, the Ve1 gene is located at the Ve locus, together with $V e 2$, a gene that similarly encodes a receptor-like protein and that has high ( $85 \%)$ sequence similarity to Ve1 (Fradin et al. 2009). Ve2 is expressed similarly as Ve1, but the encoded protein does not recognize Ave1 and thus far its function remains unknown (de Jonge et al. 2012; Fradin et al. 2009). The occurrence of Ave1 $\mathrm{L}$ alleles that encode proteins that display considerable sequence divergence, or that are severely truncated and thus likely not functional due to premature stop codons in the coding sequences, suggests that the progenitor of these alleles has experienced significant selection pressure, potentially to avoid recognition by a plant immune system. Although such selection pressure may have occurred in any plant species, considering the broad host range 
of $V$. dahliae, we tested the possibility that $\mathrm{Ve} 2$ was able to recognize ancestral forms of Ave1L. To this end, a library of potentially ancestral Ave1L alleles was constructed for co-expression with $\mathrm{Ve} 2$ in $\mathrm{N}$. tabacum. The predicted non-functional Ave $1 L$ alleles Ave1L1, Ave $1 L 3$ and Ave $1 L 4$ were chosen to reverse the disruptive mutations using site-directed mutagenesis to yield the derivatives Ave1L1*, Ave1L3* and Ave $1 L 4$ by replacing the nucleotides that are responsible for generating the stop codons by nucleotides that are present at the same positions in Ave1L2 and Ave $1 \mathrm{~L} 5$, respectively (Figure 5A and B). As Ave 1 L 3 contains multiple mutations and a retrotransposon, we synthesized Ave $1 L 3^{*}$ in which the stop codons were replaced and the retrotransposon was removed (Figure $5 \mathrm{~A}$ and $\mathrm{B}$ ). While each of the artificial derivatives (Ave1L1*, Ave1L3* and Ave1L4*) is derived from only one of the current Ave $1 \mathrm{~L}$ alleles in which only stop codons and the retrotransposon were replaced, we aimed to determine their most likely common ancestor based on the observed substitutions in the current Ave1 $\mathrm{L}$ alleles. Therefore, a consensus sequence was generated from the alignment of Ave1L alleles, leading to replacement of the various point mutations by the most frequently occurring nucleotide at the same position in the various Ave $1 \mathrm{~L}$ alleles, that we synthesized as Ave $1 L^{\star *}$ (Figure $5 \mathrm{~A}$ and B). To test the hypothesis that $\mathrm{Ve} 2$ potentially recognized any of these potentially ancestral forms of Ave1L, we co-expressed the artificially generated alleles with $\mathrm{Ve} 2$ and screened for the occurrence of a hypersensitive response. However, at five days post infiltration, no necrosis was observed in leaf tissue where Ve2 was co-expressed with either Ave1L**, Ave1L1*, Ave1L2, Ave1L3*, Ave1L4*, Ave1L5, as well as with the negative control Ave1 (Figure 5C). As expected, in leaf tissue where Ve1 was co-expressed with Ave1 as a control, clear necrosis was observed (Figure $5 \mathrm{C}$ ). Moreover, none of the Ave1 Ls is recognized by Ve1 (Figure 5C). Therefore, we conclude that it is unlikely that Ve2 recognized an Ave $1 \mathrm{~L}$ progenitor allele, and was thus responsible for its diversification. 
A

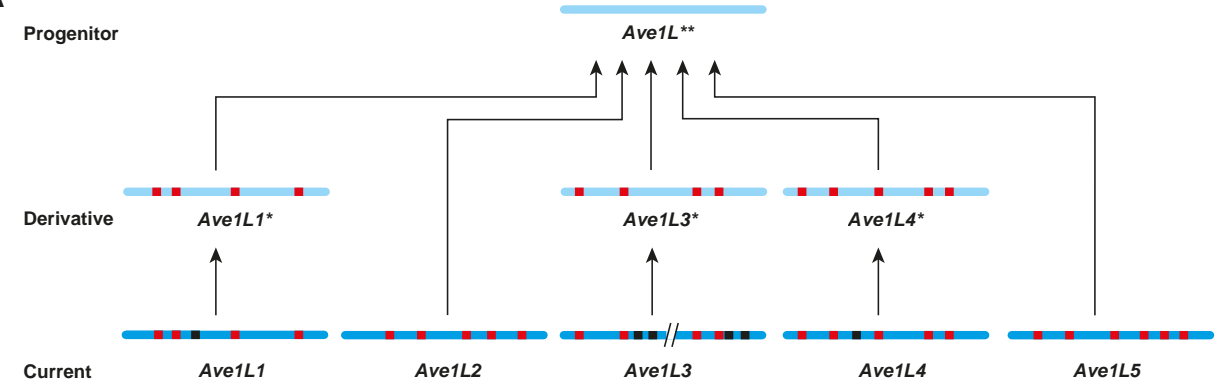

B
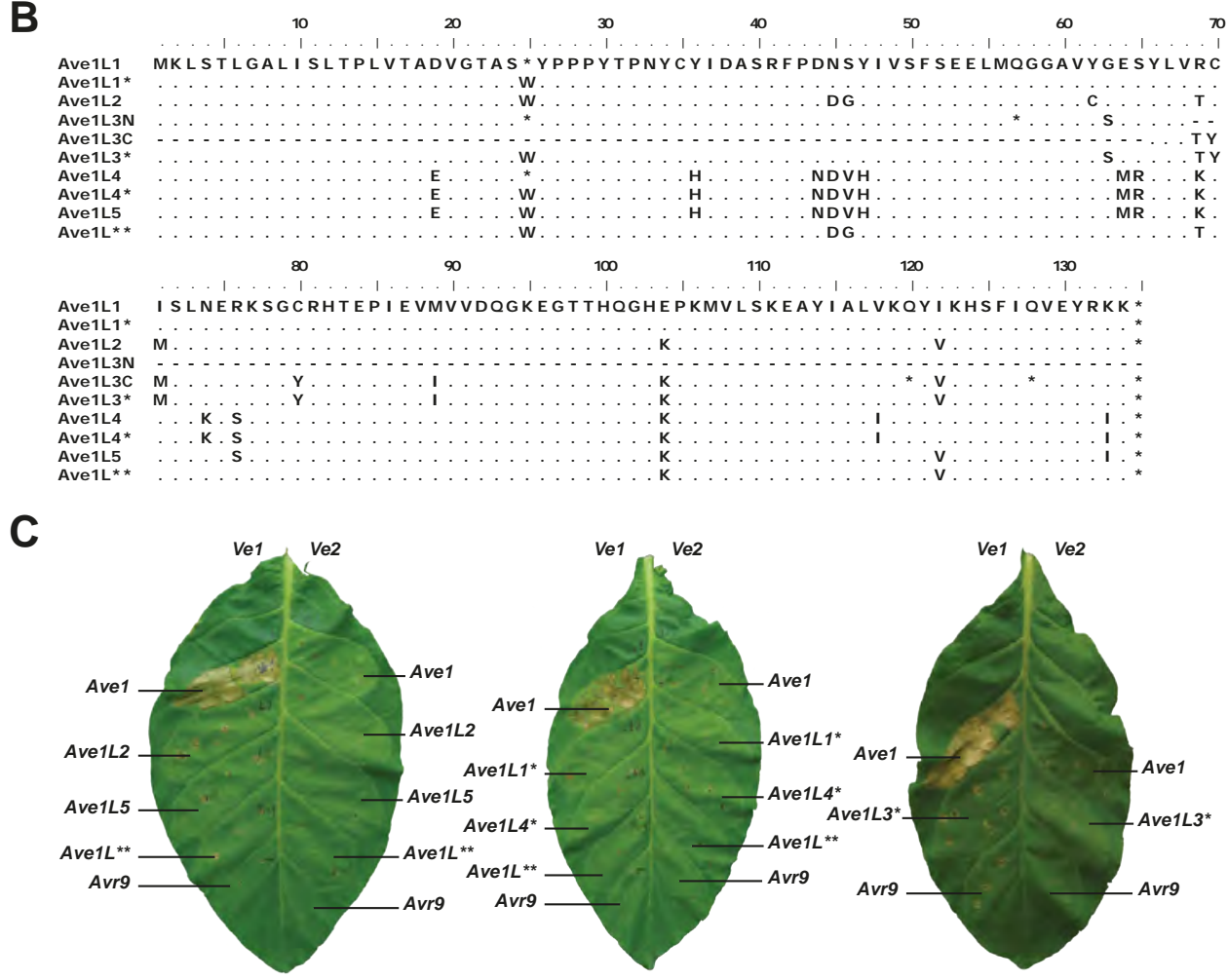

FIGURE 5 | Generation and recognition of the predicted derived alleles and the progenitor allele. (A) Schematic overview of the relationship between the currently identified Ave $1 \mathrm{~L}$ alleles (Ave1L1-Ave1L5), the predicted derived alleles Ave1L1*, Ave1L3*, Ave1L4* and the predicted progenitor allele (Ave1 $\left.L^{\star \star}\right)$. The blue bars indicate the alleles, the line with arrows indicates the relationship between the current alleles, the artificial derivatives and the progenitor. Within the blue bars the red blocks indicate point mutations, the black blocks indicate premature stop codons and the double slash (//) indicates discontiguity. (B) Alignment of Ave1 L proteins (Ave1L1-Ave1L5), derivatives (Ave1L1*, Ave1L3* and Ave1L4*), the progenitor (Ave1 $\mathrm{L}^{* *}$ ), the $\mathrm{N}$-terminal sequence of Ave1L3 (Ave1L3N) and the C-terminal sequence of Ave1L3 (Ave1L3C). Dots (.) indicate similar amino acids, lines (-) indicate the absence of an amino acid, and asterisks ( $\left.{ }^{*}\right)$ indicates a stop codon at the respective position. (C) Co-expression of Ave1L alleles with Ve1 and Ve2. The left side of the leaf is co-infiltrated with Ve1 while the right side of the leaf is co-infiltrated with Ve2. Ave 1 serves as positive control for the HR, whereas the sequence unrelated C. fulvum effector Avr 9 serves as a negative control for the HR. 


\section{Discussion}

In this study we identified a gene in V. dahliae with high similarity to the previously characterized V. dahliae effector Ave1 (de Jonge et al. 2012) that we named Ave1L. BLAST analyses of this gene in twenty sequenced $V$. dahliae strains revealed four additional alleles. These alleles follow the phylogenetic distribution of $V$. dahliae strains as previously described (de Jonge et al. 2013). Some strains do not have an Ave $1 \mathrm{~L}$ allele while other strains have a single Ave $1 \mathrm{~L}$ allele. Intriguingly, presence or absence of Ave1L does not correspond to presence or absence of Ave1. Particular strains contain Ave 1 but no Ave $1 \mathrm{~L}$ allele. Moreover, several of the alleles contain point mutations, including mutations that cause stop codons, suggesting they experienced selection pressure to inactivate the gene product. It is tempting to speculate that this pressure arose to avoid recognition by an immune receptor. However, unequivocal evidence for this hypothesis, including knowledge of the nature of the immune receptor or the host plant that carried it, remains absent today.

Since we found that Ave $1 \mathrm{~L} 2$ is expressed during host colonization we hypothesized that Ave1L2 is a virulence factor. However, Ave1L2 deletion strains of $V$. dahliae did not display attenuated virulence on tomato. The Ave1L2 deletion strains were generated in two $V$. dahliae race 1 strains that also contain Ave1. Since Ave1 makes a major contribution to virulence it cannot be excluded that redundancy in virulence functions obscures the identification of virulence defects upon Ave1L2 deletion. Unknowingly, the virulence function of Ave1 was unravelled in the JR2 strain that does not carry a functional Ave1L homolog, as the Ave1L1 homolog that is found in this strain is inactivated by an early stop codon. It can thus be explained that a potential redundancy in virulence function between Ave1 and Ave1L remained unnoticed thus far. Therefore, deletion strains should be generated in $V$. dahliae race 2 strain DVD-S26 to test the contribution to virulence in absence of Ave1.

Some virulence factors have previously been shown to be host-specific, which is particularly relevant for broad host-range pathogens such as $V$. dahliae. For example, the $V$. dahliae effector NLP2 acts as a virulence factor on Arabidopsis but not on Nicotiana benthamiana (Santhanam et al. 2013). Similarly, the EPIC effectors of Phythophthora infestans and $P$. mirabilis are only functional on their respective plant hosts (Dong et al. 2014). Arguably, Ave1L2 may act as a virulence factor on other host plants of $V$. dahliae. Potent virulence factors pose a strong selection pressure on host plants to evolve recognition (Cook et al. 2015; Thomma et al. 2011). Therefore, Ave1L2 is likely to act as a virulence factor on the host where recognition occurs. 
Ave1 is recognized by the tomato immune receptor Ve1 that is encoded by a gene at a locus that also encodes the highly identical orphan receptor Ve2. Considering the high degree of identity between the Ave 1 homologs, we tested the possibility that $\mathrm{Ve} 2$ recognized the progenitor of the current Ave $1 \mathrm{~L}$ alleles or any of the current alleles. However, the current Ave $1 \mathrm{~L}$ alleles nor the predicted progenitor or any of the intermediate alleles appears to be recognized by $\mathrm{Ve} 2$. We therefore conclude that $\mathrm{Ve} 2$ does not recognize Ave1L. The fact that $\mathrm{Ve} 2$ does not recognize Ave1L is not surprising since we do not know whether Ave $1 \mathrm{~L}$ was recognized in tomato in the first place. Moreover, if recognition occurred in tomato this may have been mediated by any of the hundreds of potential immune receptors that are encoded in the genome. However, it is not unlikely that recognition occurred in another host than tomato since $V$. dahliae has a very wide host range. The observation that Ave 1 L2 does not contribute to virulence of $V$. dahliae on tomato supports the latter hypothesis.

Ave $1 \mathrm{~L}$ and Ave1 are both located in highly dynamic lineage-specific regions of the genome, which could have contributed to the duplication of one of the two genes resulting in the other one. It has been suggested that Ave1 was acquired from plants through horizontal gene transfer (de Jonge et al. 2012) and then was lost multiple times to result in the current population of race 2 strains (Faino et al. 2016). Ave1 shows higher sequence similarity to PNPs than Ave1 L and the active PNP site appears to be degenerated and no longer functional in Ave1 (Figure 1C), whereas the PNP site in Ave1 is conserved. The sequence of Ave1 is conserved in the population and either present or absent, whereas the Ave $1 \mathrm{~L}$ alleles display variation that includes various mutations in addition to presence-absence polymorphisms. Thus, for Ave1 it has been suggested that sequence conservation is essential for its function (de Jonge et al. 2012).In addition, Ave1L alleles are present in both $V$. dahliae race 1 and race 2 strains. Collectively, these observations suggest that selection pressure on Ave1 is more recent than selection pressure on Ave1L and argues that not Ave1 but Ave $1 \mathrm{~L}$ was acquired from plants first.

\section{Materials and methods}

\section{Verticillium dahliae genomics}

Genome sequence data in this study was partly obtained from previous studies (de Jonge et al. 2013; de Jonge et al. 2012; Faino et al. 2015), and additionally contains nine newly sequenced $V$. dahliae genomes (Table 1). Library preparation (500 bp insert size) and genomic sequencing (100 bp paired-end reads) were conducted at the Beijing Genome Institute (BGl, Hong Kong, China). The genome of each individual $V$. dahliae strain was assembled using the A5 pipeline (Tritt et al. 2012). Discontiguity in a single Ave $1 \mathrm{~L}$ allele was analyzed by mapping the genomic 
paired-end sequences to the $V$. dahliae strain JR2 reference genome using BWA (Faino et al. 2015; Li and Durbin 2009).

The genetic diversity and the population structure of the sequenced $V$. dahliae strains was assessed using the reference sequence alignment based phylogeny builder (REALPHY) v. 1.11 (Bertels et al. 2014). Briefly, short paired-end genomic reads were mapped against the reference genome ( $V$. dahliae strain JR2 (Faino et al. 2015)) using Bowtie2 (Langmead and Salzberg 2012). Polymorphic as well as non-polymorphic sites were extracted (per base quality [20], coverage [10] and polymorphism frequency [0.95]) and sites that were present in all species were retained. With this pseudo-molecule, a maximum likelihood phylogenetic tree was inferred using PhyML (Guindon et al. 2009) with the generalized time reversible (GTR) nucleotide substitution model, and the robustness of the phylogeny was assessed by 500 bootstrap replicates.

\section{Ave $1 \mathrm{~L}$ sequence analysis}

To identify Ave1 $L$ sequences in the twenty $V$. dahliae genome assemblies, we used the CDS of Ave1 as a BLASTn query (Altschul et al. 1990). Sequence alignments of the five unique Ave1 $\mathrm{L}$ alleles were performed online by using Clustal Omega (EMBL-EBI, Hinxton, UK), and locally using the software package MEGA6 (Tamura et al. 2013).

Phylogeny analyses of Ave1(-like) homologs in plant pathogenic microbes and in plants was conducted using MrBayes (Huelsenbeck and Ronquist 2001). Ave1(like) homologs in plant pathogenic microbes were previously identified, and plant homologs were identified by sequence similarity search (BLASTp) against the predicted proteomes deposited in Phytozome v9.1 (Goodstein et al. 2012), supplemented by the proteome from Amborella trichopoda, and spurious sequence hits were subsequently manually filtered. Protein sequences were subsequently aligned using MAFFT (Katoh et al. 2002), and the alignment was curated using trimAl by removing alignment positions that contained $>5 \%$ gaps (Capella-Gutiérrez et al. 2009). Phylogenetic analysis was performed using MrBayes (Huelsenbeck and Ronquist 2001) with the Whelan and Goldman amino acid substitution matrix, and the gamma-shaped rate categories with a proportion of invariable sites were estimated using four rate categories. Markov Chain Monte Carlo (Marjoram et al. 2003) was run with two independent runs of four chains $(T=0.1)$ and a sample frequency of 500 for 40,000,000 generations. At this point, the average standard deviation of split frequency, a measure of convergence, was well below 0.02. The first $25 \%$ of samples of each run were discarded as burn-in, and the remaining samples were used to infer tree and branch topology. 


\section{Ave 1 L gene expression and gene deletions}

Arabidopsis (Arabidopsis thaliana) ecotype Col-0 and tomato (Solanum lycopersicum) cultivar MoneyMaker were inoculated with V. dahliae strains VdLs17 (containing Ave1L1), DVD-S26 (Ave1L2), St14.01 (Ave1L2), DVD-S94 (Ave1L3), DVD-S29 (Ave1L4) and St.100 (Ave1L5) as previously described (Fradin et al. 2011; Fradin et al. 2009). The stems of tomato plants were cut from the base to the first cotyledons and flash frozen in liquid nitrogen. The base of Arabidopsis plants of which the leaves were removed were harvested and flash frozen in liquid nitrogen. Plant material was ground to a powder and RNA was extracted using Quick-RNA ${ }^{\text {TM }}$ Miniprep (Zymo Research Europe GmbH, Freiburg, Germany) and cDNA was synthesized using M-MLV Reverse Transcriptase (Promega Benelux BV, Leiden, The Netherlands). Real-time PCR was conducted using an ABI7300 PCR machine (Applied Biosystems, Life Technologies Europe BV, Bleiswijk, The Netherlands) in combination with the GPCR SensiMix kit (BioLine, GC Biotech BV, Alphen aan den Rijn, The Netherlands). Primers that were used for gene expression and fungal biomass are described in supplemental table 2. Real-time PCR conditions were as follows: an initial $95^{\circ} \mathrm{C}$ denaturation step for 10 minutes followed by denaturation for 15 seconds at $95^{\circ} \mathrm{C}$, annealing for 60 seconds at $60^{\circ} \mathrm{C}$, and extension at $72^{\circ} \mathrm{C}$ for 40 cycles. Gene expression and fungal biomass was analyzed using the software package 7300 System Software (Applied Biosystems, Life Technologies Europe BV, Bleiswijk, The Netherlands). To generate Ave1L2 deletion strains, primers were designed to amplify approximately $1500 \mathrm{bp}$ up- and downstream of the Ave1L2 CDS (Supplemental table 2). Both amplicons were used to generate a USER friendly cloning construct to replace Ave 1 L2 by a hygromycin cassette through homologous recombination (Frandsen et al. 2008).

\section{Production of derived Ave $1 L$ alleles}

The potential progenitor of the Ave $1 \mathrm{~L}$ alleles was obtained by generating a consensus sequence from an alignment of the CDS of Ave1L alleles and was synthesized as Ave1L**. The predicted derivatives of Ave1L1 and Ave1L4 were obtained by replacing the predicted premature stop codons. To this end primers were designed (Supplemental table 2) to replace 1 nucleotide in the stop codon with a nucleotide at the same position in Ave 1 L2 and Ave1L5 that do not contain a predicted premature stop codon. Overlap PCR was performed to introduce the mutation in Ave1L1 and Ave1L4. The Ave1L1 and Ave1L4 sequences without a predicted premature stop codon were designated as Ave1L1* and Ave1L4*, respectively (Figure $4 A$ ). The derivative of Ave $1 \mathrm{L3}$ was obtained by synthesizing the gene without stop codons and without the retrotransposon and was designated as Ave1L3* (Eurofins Genomics, Ebersberg, Germany) (Figure 4A). 


\section{Functional analysis of Ave $1 \mathrm{~L}$ alleles}

V. dahliae strains were grown on potato dextrose agar (PDA) (Thermo Fisher Scientific Inc, Breda, The Netherlands) for 1-2 weeks and conidia were harvested for DNA extraction with high-salt CTAB buffer (100 mM TrisHCl, 20 mM EDTA, 2 M $\mathrm{NaCl}, 3 \% \mathrm{CTAB})$. PCR primers were designed to amplify Ave1, Ave1L or to detect discontiguity (Supplemental table 2). For constitutive expression the CDS of Ave1L alleles was amplified from genomic DNA and cloned in the adjusted, Gateway compatible, pBIN vector variant pSol2092 (Zhang et al. 2013) and transformed into Agrobacterium tumefaciens strain GV3101. Nicotiana tabacum cv. Petite Havana SR1 was infiltrated in a 1:1 ratio with $A$. tumefaciens that express either Ve1 or Ve2 and an Ave1(-like) construct, as previously described (Zhang et al. 2013). After infiltration, plants were transferred to a climate chamber and incubated at $22^{\circ} \mathrm{C}$ and $19^{\circ} \mathrm{C}$ during $16-\mathrm{h}$ day and 8 -h night periods, respectively, with $70 \%$ relative humidity. At 5 dpi leaves were inspected for necrosis development.

\section{Oxford Nanopore MinION sequencing}

Library preparation of the PCR fragment was performed according to the protocol of Oxford Nanopore (https://wiki.nanoporetech.com/display/BP/Nanopore+Sequenci ng+Kit+protocol+SOK-MAP005) skipping the DNA fragmentation step. The library was loaded on the Nanopore flowcell and the sequencing was stopped after $\sim 4$ hr. The run generated about 870 high quality long reads with an average length of 3,869 bp and the longest read of about $14 \mathrm{~kb}$. Using Nanocorrect software (https:// github.com/jts/nanocorrect), we used all reads to correct the longest 50 reads. Out of the 50 longest reads, only 28 were corrected to generate a consensus. The average protected read length was of 5,666.8 bp with a median of 6,483 bp. All the reads were used in BLAST analysis to confirm the presence of Ave $1 \mathrm{~L} 3$ fragments at both ends. Out of the 28 corrected fragments, eight showed high similarity ( 99\%) to Ave 1 L3 at both ends.

\section{Acknowledgements}

We thank Bert Essenstam and Pauline Sanderson at Unifarm for excellent plant care. We thank Jose Espejo Valle-Inclan for assistance with the Oxford Nanopore MinION sequencing. We thank Dorien Oppelaar for technical assistance in the lab. 


\section{References}

Altschul, S. F., Gish, W., Miller, W., Myers, E. W., and Lipman, D. J. 1990. Basic local alignment search tool. J Mol Biol 215:403-410.

Bertels, F., Silander, O. K., Pachkov, M., Rainey, P. B., and van Nimwegen, E. 2014. Automated reconstruction of whole-genome phylogenies from short-sequence reads. Mol Biol Evol 31:10771088.

Bolton, M. D., van Esse, H. P., Vossen, J. H., de Jonge, R., Stergiopoulos, I., Stulemeijer, I. J., van den Berg, G. C., Borras-Hidalgo, O., Dekker, H. L., de Koster, C. G., de Wit, P. J. G. M., Joosten, M. H., and Thomma, B. P. H. J. 2008. The novel Cladosporium fulvum lysin motif effector Ecp6 is a virulence factor with orthologues in other fungal species. Mol Microbiol 69:119-136.

Capella-Gutiérrez, S., Silla-Martínez, J. M., and Gabaldón, T. 2009. trimAl: a tool for automated alignment trimming in large-scale phylogenetic analyses. Bioinformatics 25:1972-1973.

Cook, D. E., Mesarich, C. H., and Thomma, B. P. H. J. 2015. Understanding plant immunity as a surveillance system to detect invasion. Annu Rev Phytopathol.

de Jonge, R., Bolton, M. D., Kombrink, A., van den Berg, G. C., Yadeta, K. A., and Thomma, B. P. H. J. 2013. Extensive chromosomal reshuffling drives evolution of virulence in an asexual pathogen. Genome Res 23:1271-1282.

de Jonge, R., van Esse, H. P., Maruthachalam, K., Bolton, M. D., Santhanam, P., Saber, M. K., Zhang, Z., Usami, T., Lievens, B., Subbarao, K. V., and Thomma, B. P. H. J. 2012. Tomato immune receptor Ve1 recognizes effector of multiple fungal pathogens uncovered by genome and RNA sequencing. Proc Natl Acad Sci U S A 109:5110-5115.

de Wit, P. J. 2016. Cladosporium fulvum Effectors: Weapons in the Arms Race with Tomato. Annu Rev Phytopathol 54:1-23.

Dodds, P. N., and Rathjen, J. P. 2010. Plant immunity: towards an integrated view of plant-pathogen interactions. Nat Rev Genet 11:539-548.

Dong, S., Stam, R., Cano, L. M., Song, J., Sklenar, J., Yoshida, K., Bozkurt, T. O., Oliva, R., Liu, Z., Tian, M., Win, J., Banfield, M. J., Jones, A. M., van der Hoorn, R. A., and Kamoun, S. 2014. Effector specialization in a lineage of the Irish potato famine pathogen. Science 343:552-555.

Faino, L., Seidl, M. F., Datema, E., van den Berg, G. C., Janssen, A., Wittenberg, A. H., and Thomma, B. P. H. J. 2015. Single-Molecule Real-Time Sequencing Combined with Optical Mapping Yields Completely Finished Fungal Genome. MBio 6.

Faino, L., Seidl, M. F., Shi-Kunne, X., Pauper, M., van den Berg, G. C., Wittenberg, A. H., and Thomma, B. P. 2016. Transposons passively and actively contribute to evolution of the two-speed genome of a fungal pathogen. Genome Res 26:1091-1100.

Fradin, E. F., and Thomma, B. P. H. J. 2006. Physiology and molecular aspects of Verticillium wilt diseases caused by V. dahliae and V. albo-atrum. Mol Plant Pathol 7:71-86.

Fradin, E. F., Abd-El-Haliem, A., Masini, L., van den Berg, G. C., Joosten, M. H., and Thomma, B. P. H. J. 2011. Interfamily transfer of tomato Ve1 mediates Verticillium resistance in Arabidopsis. Plant Physiol 156:2255-2265.

Fradin, E. F., Zhang, Z., Juarez Ayala, J. C., Castroverde, C. D., Nazar, R. N., Robb, J., Liu, C. M., and Thomma, B. P. H. J. 2009. Genetic dissection of Verticillium wilt resistance mediated by tomato Ve1. Plant Physiol 150:320-332.

Frandsen, R. J., Andersson, J. A., Kristensen, M. B., and Giese, H. 2008. Efficient four fragment cloning for the construction of vectors for targeted gene replacement in filamentous fungi. BMC Mol Biol 9:70.

Garavaglia, B. S., Thomas, L., Gottig, N., Dunger, G., Garofalo, C. G., Daurelio, L. D., Ndimba, B., Orellano, E. G., Gehring, C., and Ottado, J. 2010. A eukaryotic-acquired gene by a biotrophic phytopathogen allows prolonged survival on the host by counteracting the shut-down of plant photosynthesis. PLoS One 5:e8950. 
Gehring, C., and Irving, H. 2003. Natriuretic peptides - a class of heterologous molecules in plants. Int J Biochem Cell Biol 35:1318-1322.

Goodstein, D. M., Shu, S., Howson, R., Neupane, R., Hayes, R. D., Fazo, J., Mitros, T., Dirks, W., Hellsten, U., and Putnam, N. 2012. Phytozome: a comparative platform for green plant genomics. Nucleic Acids Res 40:D1178-D1186.

Gottig, N., Garavaglia, B. S., Daurelio, L. D., Valentine, A., Gehring, C., Orellano, E. G., and Ottado, J. 2008. Xanthomonas axonopodis pv. citri uses a plant natriuretic peptide-like protein to modify host homeostasis. Proc Natl Acad Sci U S A 105:18631-18636.

Guindon, S., Delsuc, F., Dufayard, J.-F., and Gascuel, O. 2009. Estimating maximum likelihood phylogenies with PhyML. Pages 113-137 in: Bioinformatics for DNA Sequence Analysis. Springer.

Houterman, P. M., Cornelissen, B. J., and Rep, M. 2008. Suppression of plant resistance gene-based immunity by a fungal effector. PLoS Pathog 4:e1000061.

Huelsenbeck, J. P., and Ronquist, F. 2001. MRBAYES: Bayesian inference of phylogenetic trees. Bioinformatics 17:754-755.

Joosten, M., Vogelsang, R., Cozijnsen, T. J., Verberne, M. C., and de Wit, P. J. G. M. 1997. The biotrophic fungus Cladosporium fulvum circumvents $\mathrm{Cf}$-4-mediated resistance by producing unstable AVR4 elicitors. Plant Cell 9:367-379.

Joosten, M. H., Cozijnsen, T. J., and de Wit, P. J. G. M. 1994. Host resistance to a fungal tomato pathogen lost by a single base-pair change in an avirulence gene. Nature 367:384-386.

Katoh, K., Misawa, K., Kuma, K. i., and Miyata, T. 2002. MAFFT: a novel method for rapid multiple sequence alignment based on fast Fourier transform. Nucleic Acids Res 30:3059-3066.

Kawchuk, L. M., Hachey, J., Lynch, D. R., Kulcsar, F., van Rooijen, G., Waterer, D. R., Robertson, A., Kokko, E., Byers, R., Howard, R. J., Fischer, R., and Prufer, D. 2001. Tomato Ve disease resistance genes encode cell surface-like receptors. Proc Natl Acad Sci U S A 98:6511-6515.

Klosterman, S. J., Subbarao, K. V., Kang, S., Veronese, P., Gold, S. E., Thomma, B. P. H. J., Chen, Z., Henrissat, B., Lee, Y. H., Park, J., Garcia-Pedrajas, M. D., Barbara, D. J., Anchieta, A., de Jonge, R., Santhanam, P., Maruthachalam, K., Atallah, Z., Amyotte, S. G., Paz, Z., Inderbitzin, P., Hayes, R. J., Heiman, D. I., Young, S., Zeng, Q., Engels, R., Galagan, J., Cuomo, C. A., Dobinson, K. F., and Ma, L. J. 2011. Comparative genomics yields insights into niche adaptation of plant vascular wilt pathogens. PLoS Pathog 7:e1002137.

Langmead, B., and Salzberg, S. L. 2012. Fast gapped-read alignment with Bowtie 2. Nat Methods 9:357359.

Lauge, R., Goodwin, P. H., de Wit, P. J. G. M., and Joosten, M. H. 2000. Specific HR-associated recognition of secreted proteins from Cladosporium fulvum occurs in both host and non-host plants. Plant $\mathrm{J}$ 23:735-745.

Li, H., and Durbin, R. 2009. Fast and accurate short read alignment with Burrows-Wheeler transform. Bioinformatics 25:1754-1760.

Luderer, R., Takken, F. L., de Wit, P. J. G. M., and Joosten, M. H. 2002. Cladosporium fulvum overcomes Cf-2-mediated resistance by producing truncated AVR2 elicitor proteins. Mol Microbiol 45:875884.

Ludidi, N., Morse, M., Sayed, M., Wherrett, T., Shabala, S., and Gehring, C. 2004. A recombinant plant natriuretic peptide causes rapid and spatially differentiated $\mathrm{K}+, \mathrm{Na}+$ and $\mathrm{H}+$ flux changes in Arabidopsis thaliana roots. Plant Cell Physiol 45:1093-1098.

Marjoram, P., Molitor, J., Plagnol, V., and Tavaré, S. 2003. Markov chain Monte Carlo without likelihoods. Proc Natl Acad Sci U S A 100:15324-15328.

Maryani, M., Bradley, G., Cahill, D., and Gehring, C. 2001. Natriuretic peptides and immunoreactants modify osmoticum-dependent volume changes in Solanum tuberosum L. mesophyll cell protoplasts. Plant Sci 161:443-452.

Mesarich, C. H., Griffiths, S. A., van der Burgt, A., Okmen, B., Beenen, H. G., Etalo, D. W., Joosten, M. H., and de Wit, P. J. G. M. 2014. Transcriptome sequencing uncovers the Avr5 avirulence gene of the tomato leaf mold pathogen Cladosporium fulvum. Mol. Plant Microbe Interact. 27:846-857. 
Morse, M., Pironcheva, G., and Gehring, C. 2004. AtPNP-A is a systemically mobile natriuretic peptide immunoanalogue with a role in Arabidopsis thaliana cell volume regulation. FEBS Lett 556:99-103.

Pharmawati, M., Maryani, M. M., Nikolakopoulos, T., Gehring, C. A., and Irving, H. R. 2001. Cyclic GMP modulates stomatal opening induced by natriuretic peptides and immunoreactive analogues. Plant Physiol Biochem 39:385-394.

Rovenich, H., Boshoven, J. C., and Thomma, B. P. H. J. 2014. Filamentous pathogen effector functions: of pathogens, hosts and microbiomes. Curr Opin Plant Biol 20:96-103.

Santhanam, P., van Esse, H. P., Albert, I., Faino, L., Nurnberger, T., and Thomma, B. P. H. J. 2013. Evidence for functional diversification within a fungal NEP1-like protein family. Mol. Plant Microbe Interact. 26:278-286.

Seidl, M. F., and Thomma, B. P. H. J. 2014. Sex or no sex: Evolutionary adaptation occurs regardless. BioEssays 36:335-345.

Stergiopoulos, I., De Kock, M. J., Lindhout, P., and de Wit, P. J. G. M. 2007. Allelic variation in the effector genes of the tomato pathogen Cladosporium fulvum reveals different modes of adaptive evolution. Mol. Plant Microbe Interact. 20:1271-1283.

Tamura, K., Stecher, G., Peterson, D., Filipski, A., and Kumar, S. 2013. MEGA6: molecular evolutionary genetics analysis version 6.0. Mol Biol Evol 30:2725-2729.

Thomma, B. P. H. J., Nurnberger, T., and Joosten, M. H. A. J. 2011. Of PAMPs and effectors: The blurred PTI-ETI dichotomy. Plant Cell 23:4-15.

Thomma, B. P. H. J., van Esse, H. P., Crous, P. W., and de Wit, P. J. G. M. 2005. Cladosporium fulvum (syn. Passalora fulva), a highly specialized plant pathogen as a model for functional studies on plant pathogenic Mycosphaerellaceae. Mol Plant Pathol 6:379-393.

Tritt, A., Eisen, J. A., Facciotti, M. T., and Darling, A. E. 2012. An integrated pipeline for de novo assembly of microbial genomes. PLoS One 7:e42304.

Van den Ackerveken, G. F., Van Kan, J. A., Joosten, M. H., Muisers, J. M., Verbakel, H. M., and de Wit, P. J. G. M. 1993. Characterization of two putative pathogenicity genes of the fungal tomato pathogen Cladosporium fulvum. Mol. Plant Microbe Interact. 6:210-215.

van Kan, J. A., van den Ackerveken, G., and de Wit, P. J. G. M. 1991. Cloning and characterization of cDNA of avirulence gene avr9 of the fungal pathogen Cladosporium fulvum, causal agent of tomato leaf mold. Mol. Plant Microbe Interact. 4:52-59.

Westerink, N., Brandwagt, B. F., de Wit, P. J. G. M., and Joosten, M. H. A. J. 2004. Cladosporium fulvum circumvents the second functional resistance gene homologue at the Cf-4 locus (Hcr9-4E) by secretion of a stable avr4E isoform. Mol Microbiol 54:533-545.

Zhang, Z., Fradin, E., de Jonge, R., van Esse, H. P., Smit, P., Liu, C.-M., and Thomma, B. P. H. J. 2013. Optimized agroinfiltration and virus-induced gene silencing to study Ve1-mediated Verticillium resistance in tobacco. Mol. Plant Microbe Interact. 26:182-190. 


\section{Supplemental data}

SUPPLEMENTAL TABLE 2 | Primers used in this study

\begin{tabular}{|c|c|c|}
\hline Primer & Sequence $\left(5^{\prime}-3^{\prime}\right)$ & Notes \\
\hline Ave1L-Break-Fw & TCGATGCGAGCAGATTCC & Primers spanning discontiguity \\
\hline Ave1L-Break-Rv & GAGTAGTACCTTCCTTGCCTTGA & Primers spanning discontiguity \\
\hline Ave1L-Up-Fw & CTACGCTTGGAGCCCTCAT & Primers upstream of discontiguity \\
\hline Ave1L-Up-Rv & AGGAGTGTAGGGTGGAGGAT & Primers upstream of discontiguity \\
\hline Ave1L-Down-Fw & GGCATACTGAACCGATTGAAG & Primers downstream of discontiguity \\
\hline Ave1L-Down-Rv & CTCCTTAGAAAGAACCATTTTCG & Primers downstream of discontiguity \\
\hline VdLs17del.stop_Fw & CGCATCCTGGTATCCTCCACC & Replace stop codon in Ave $1 L 1$ and Ave $1 L 4$ \\
\hline VdLs17del.stop_Rv & TGGAGGATACCAGGATGCGGT & Replace stop codon in Ave $1 L 1$ and Ave $1 L 4$ \\
\hline Topo_Ave1L_Fw & CACCATGAAGCTTTCTACGCTTGGAG & Combined with primer VdLs17del.stop_Rv \\
\hline Topo_St14.01_Rv & TTATTTTTTTCTATACTCAACTTGAATGAAC & Combined with primer VdLs17del.stop_Fw \\
\hline Topo_St.100_Rv & TTATTTTATTCTATACTCAACTTGAATGAAC & Combined with primer VdLs17del.stop_Fw \\
\hline Ave1L2-LB-F3 & GGTCTTAAUTTTTATCTCTCCCTTCCTCTTATCT & $1500 \mathrm{bp}$ fragment upstream of Ave $1 \mathrm{L2}$ \\
\hline Ave1L2-LB-R3 & GGCATTAAUTTTTTAAGCCTTTCTAGCTTATTCTT & 1500 bp fragment upstream of Ave $1 L 2$ \\
\hline Ave1L2-RB-F3 & GGACTTAAUGCTATCTTCACGAGAGCAGAGT & 1500bp fragment downstream of Ave $1 \mathrm{~L} 2$ \\
\hline Ave1L2-RB-R3 & GGGTTTAAUTTGCGCGTTTATATATTCTTATCTT & 1500bp fragment downstream of Ave $1 L 2$ \\
\hline QPCR-Ave1L-Fw3 & АТССТССАСССТАСАСТССТ & Ave $1 \mathrm{~L}$ expression \\
\hline QPCR-Ave1L-Rv3 & CCTTGATGAGTAGTACCTTCCTTG & Ave1L expression \\
\hline Vd-ITS-Fw & AAAGTTTTAATGGTTCGCTAAGA & V. dahliae biomass \\
\hline Vd-ITS-Rv & CTTGGTCATTTAGAGGAAGTAA & V. dahliae biomass \\
\hline SI-RUB-Fw & GAACAGTTTCTCACTGTTGAC & Tomato rubisco gene \\
\hline SI-RUB-Rv & CGTGAGAACCATAAGTCACC & Tomato rubisco gene \\
\hline
\end{tabular}

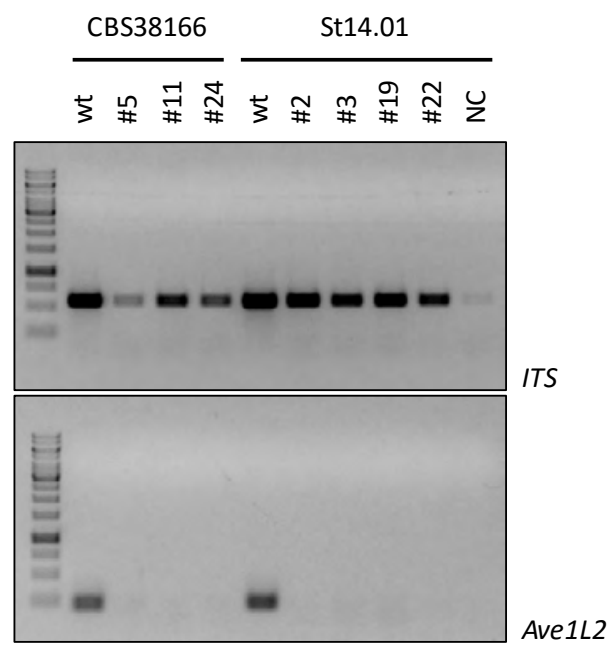

SUPPLEMENTAL FIGURE 1 | Verification of Ave 1 L2 deletion strains by amplification of VdITS and Ave1L2 from V. dahliae strains CBS38166 and St14.01. 

Rhamnose synthase activity is required for pathogenicity of the vascular wilt fungus Verticillium dahliae

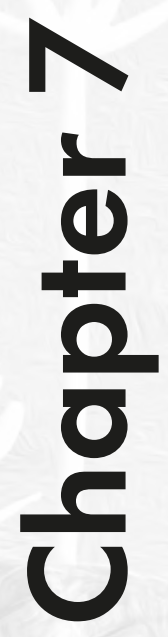

Parthasarathy Santhanam

Jordi C. Boshoven\#

Omar Salas ${ }^{\#}$

Kyle Bowler"

Md Tohidul Islam

Mojtaba Keykha Saber

Grardy C.M. van den Berg

Maor Bar-Peled

Bart P.H.J. Thomma

\#These authors contributed equally 
The initial interaction of a pathogenic fungus with its host is complex and involves numerous metabolic pathways and regulatory proteins. Here we present a random genetic screen that enabled us to identify 58 novel candidate genes that are involved in the pathogenic potential of the fungal pathogen Verticillium dahliae that causes vascular wilt diseases in over 200 dicotyledonous plant species, including economically important crops. One of the candidate genes that was identified concerns a putative biosynthetic gene involved in nucleotidesugar precursor formation as it encodes a putative nucleotide-rhamnose synthase/epimerase-reductase (NRS/ER). This enzyme has homology to bacterial enzymes involved in biosysnthesis of the nucleotide sugar dTDP-rhamnose, a precursor of L-rhamnose that was shown to be required for virulence in several human pathogenic bacteria. Rhamnose is known as a minor cell wall glycan in fungi and has therefore not been suspected as a crucial molecule in fungal-host interactions. Nevertheless, our study shows that deletion of the VdNRS/ER gene from the $V$. dahliae genome results in complete loss of pathogenicity on tomato and $N$. benthamiana plants, whereas vegetative growth and sporulation are not affected. We demonstrate that VdNRS/ER is a functional enzyme in the biosynthesis of UDP-rhamnose, and further analysis revealed that $V d N R S / E R$ deletion strains are impaired in colonization of tomato roots. Collectively, our results demonstrate that rhamnose, although only being a minor cell wall component, is essential for pathogenicity of $V$. dahliae. 


\section{Introduction}

Vascular wilt diseases caused by soil-borne pathogens are among the most devastating plant diseases worldwide. The fungus Verticillium dahliae causes vascular wilt diseases in over 200 dicotyledonous plant species, including important crops such as eggplant, lettuce, olive, spinach and tomato (Fradin and Thomma 2006; Klosterman et al. 2009). Triggered by root exudates the melanised resting structures, microsclerotia, germinate and penetrate roots through the root tips, wounds, or sites of lateral root formation (Fradin and Thomma 2006). After crossing the cortex, hyphae of the fungus grow into the xylem vessels. The mycelium remains exclusively in these vessels and produces conidia which are transported acropetally with the water flow in the xylem throughout the plant. Once senescing, microsclerotia are produced that are released into the soil upon tissue decomposition (Wilhelm 1955). Typical symptoms of plants infected with V. dahliae comprise stunting, wilting, chlorosis and vascular browning (Pegg and Brady 2002).

Verticillium wilt diseases are difficult to control due to the longevity of the microsclerotia and inability of fungicides to eliminate the fungus once it has entered the xylem tissues of the host plant (Fradin and Thomma 2006; Wilhelm 1955). Moreover, crop rotation is ineffective due to the broad host range of $V$. dahliae. The only effective control measure, soil fumigation, is expensive and has harmful environmental effects (Rowe et al. 1987). As a consequence, genetic resistance is presently preferred to control Verticillium wilt diseases. Importantly, Verticillium resistance has been described in several plant species, including crops (Bolek et al. 2005; Hayes et al. 2011; Schaible et al. 1951; Simko et al. 2004). From tomato (Solanum lycopersicum) the Ve1 Verticillium resistance gene has been cloned that mediates resistance to race 1 strains of $V$. dahliae that express the secreted Ave 1 effector (de Jonge et al. 2012; Fradin et al. 2009). Putative Ve1 orthologs have been identified within and outside the Solanaceae family, suggesting that Ve1mediated Verticillium resistance may be widespread in plants (Thomma et al. 2011). Interestingly, Ave1 contributes to V. dahliae virulence on susceptible tomato plants, and homologs occur in a handful of taxonomically unrelated plant pathogenic fungi (de Jonge et al. 2012).

Various other virulence genes have been identified in $V$. dahliae including members of the expanded LysM effector and NEP-like (NLP) protein family (de Jonge et al. 2013; Klimes et al. 2015; Klosterman et al. 2011; Santhanam et al. 2013). Genome analysis furthermore revealed the relative abundance of particular families of cellwall degrading enzymes (CWDE) when compared with other fungi (Klosterman et al. 2011). The importance of CWDE during pathogenicity was demonstrated by the disruption of the CWDE regulator VdSNF1 which reduced $V$. dahliae virulence (Tzima 
et al. 2011). In addition, genes involved in nitrogen and amino acid metabolism where found to be important for establishment in the nutrient-poor vascular system (Singh et al. 2010; Timpner et al. 2013). Finally, virulence genes were described that are also involved in stress tolerance (Klimes et al. 2015).

The identification and subsequent characterization of novel genes involved in disease establishment is of fundamental importance to understand the interaction between $V$. dahliae and its hosts, and to potentially design novel strategies for disease control. An effective way to identify novel pathogenicity genes in plant pathogenic fungi is the use of random mutagenesis coupled with a quick manner to screen the mutants for phenotypes. Agrobacterium tumefaciens is a Gram-negative soil bacterium that can infect hosts as diverse as fungi, oomycetes and plants, and can be used to randomly insert a transfer-DNA (T-DNA) into the host genome. Over recent years, A. tumefaciens-mediated transformation (ATMT) was used to identify genes involved in disease development in various plant pathogens (Giesbert et al. 2012; Hüser et al. 2009; Jeon et al. 2007; Korn et al. 2015; Maruthachalam et al. 2011; Michielse et al. 2009; Munch et al. 2011; Ramos et al. 2013; Schumacher et al. 2014; Xu and Chen 2013). In this study, we report on the identification of pathogenicity and virulence genes in $V$. dahliae through ATMT. A collection of 900 transformants of $V$. dahliae was generated and tested for reduced disease development on susceptible tomato plants. We subsequently studied one of the candidates in detail, a gene that putatively encodes a biosynthetic gene involved in nucleotide-sugar precoursor formation, to reveal its role in pathogenicity.

\section{Results}

\section{Identification of random V. dahliae transformants affected in aggressiveness}

In this study, we generated 900 random T-DNA insertion mutants of $V$. dahliae by transforming conidiospores through ATMT. All transformants were assessed for reduced potential to cause disease on tomato seedlings by root-dip inoculation and scored for typical disease symptoms, including stunting, wilting, chlorosis and necrosis, for up to 14 days post inoculation (dpi). Seedlings that exhibited reduced Verticillium wilt symptoms when compared with plants inoculated with wild type $V$. dahliae were selected, and the corresponding $V$. dahliae mutants were retained for rescreening, leading to a set of 200 transformants. Subsequently, after calibration of the conidial concentration in the inoculum to $10^{6}$ conidia/mL, all 200 transformants were reassessed for compromised ability to cause wilt disease on tomato plants for up to $21 \mathrm{dpi}$. Eventually, 80 transformants showed a reproducible defect in disease development when compared with the wild-type progenitor strain (Figure 1). 


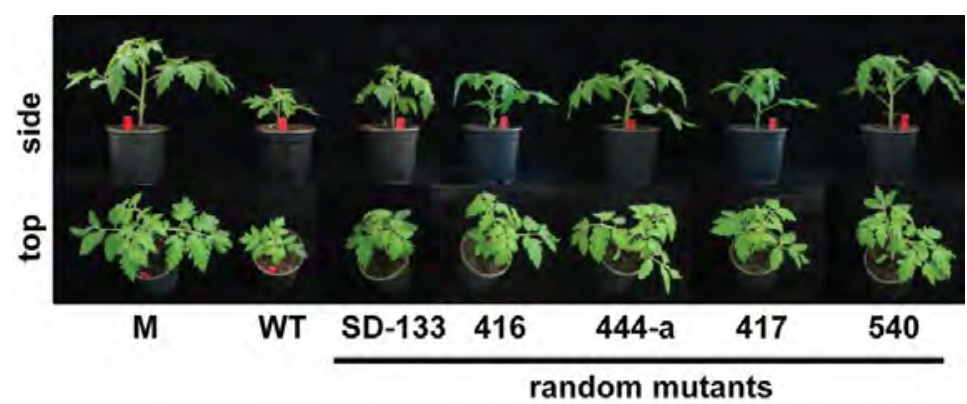

FIGURE 1 | Typical assay to identify transformants with reduced pathogenicity or virulence. Ten-day-old tomato seedlings were mock-inoculated (M) or inoculated with conidiospores of wildtype V. dahliae (WT) or random T-DNA insertion mutants. At 21 days post inoculation the plants were scored for disease development by comparing plants inoculated with wild-type $V$. dahliae with those inoculated with the T-DNA insertion mutants. Side (upper panel) and top (bottom panel) of plants inoculated with five T-DNA insertion mutants that are impaired in aggressiveness (upper panel) and the top (bottom panel).

\section{Isolation of T-DNA flanking regions in selected V. dahliae transformants}

Inverse PCR (iPCR) was carried out on the 80 selected transformants to determine the T-DNA insertion sites. The genomic borders flanking the T-DNA were amplified, sequenced, and the resulting sequences were queried against the $V$. dahliae genome (Faino et al. 2015). In this manner, sequences flanking the T-DNA were determined for 65 mutants, while T-DNA flanking regions repeatedly could not be amplified from any of the borders of the remaining 15 mutants. Of the 65 flanking sequences, 12 sequences corresponded to the backbone of the vector that was used for ATMT while 10 sequences could not unambiguously be assigned to a single genomic location. The remaining 43 sequences resulted in single genomic hits, allowing determination of T-DNA integration sites. In total, 14 insertions were found within predicted open reading frames (ORFs; Table 1) of which two transformants carried an insertion in the same ORF, while the remaining 29 insertions occurred in noncoding regions. For these 29 insertions the predicted genes flanking the T-DNA were identified. In one case we found that for two transformants the T-DNA was integrated in the same non-coding region, albeit at a slightly different location, resulting in the identification of the same flanking genes. In another case the same candidate gene was identified twice in two transformants due to an insertion upstream and downstream of the gene. One flanking gene was only identified at $55,077 \mathrm{bp}$ from the insertion site, for which we disqualified it as a candidate gene. In total, this resulted in the identification of 55 candidate genes from the 29 mutants with insertions in non-coding regions. 
In order to try to determine which of the two genes flanking a particular insertion site in the non-coding region is likely causal to the reduced disease development, in planta expression of the flanking genes was assessed making use of previously generated RNAseq data of $V$. dahliae-inoculated Nicotiana benthamiana plants (de Jonge et al. 2012; Faino et al. 2012). For 10 insertions, this revealed that only one of the two flanking genes is expressed during infection, disqualifying the 10 candidate genes that are not expressed. Thus, 45 candidate genes were considered from mutants with an insertion in the non-coding region and 13 candidate genes were identified from mutants with an insertion in the ORF (Table 1). Subsequently, the 58 candidate genes were queried against the pathogen-host interaction (PHI) database that contains experimentally verified pathogenicity and virulence factors of plant and animal pathogens (Winnenburg et al. 2006; Winnenburg et al. 2008), which resulted in the identification of homologs $\left(E<10^{-6}\right)$ for only 19 of the 58 candidate genes (Table 2).

TABLE 1 | List of candidate genes identified based on the T-DNA insertion sites in the random mutants that showed compromised virulence on tomato.

\begin{tabular}{|c|c|c|c|c|}
\hline \multicolumn{5}{|c|}{ T-DNA insertion within the CDS } \\
\hline MutantOld locus ${ }^{b}$ & New locus ${ }^{c}$ & Annotation & Expr. & $D^{e}$ \\
\hline 78 Not available & VDAG_JR2_Chr1g08240 & glycoside hydrolase & + & - \\
\hline 73 Not available & VDAG_JR2_Chr1g08240 & glycoside hydrolase & + & - \\
\hline 407 VDAG_00500 & VDAG_JR2_Chr1g19180 & carboxypeptidase d & + & - \\
\hline 659 VDAG_02200 & VDAG_JR2_Chr2g03300 & alpha-beta hydrolase fold family & - & - \\
\hline SD-133 VDAG_04418 & VDAG_JR2_Chr3g02140 & ran gtp-ase activating protein 1 & + & - \\
\hline 444-B VDAG_08830 & VDAG_JR2_Chr3g07650 & acetate kinase & + & - \\
\hline 211 VDAG_06985 & VDAG_JR2_Chr4g06020 & leucine rich-repeat protein & + & - \\
\hline 375 VDAG_07314 & VDAG_JR2_Chr4g10770 & copper amine oxidase 1 & + & - \\
\hline 416 VDAG_10292 & VDAG_JR2_Chr5g06230 & conserved hypothetical protein & - & - \\
\hline 174 VDAG_05684 & VDAG_JR2_Chr5g09640 & hypothetical protein & + & - \\
\hline 657-2 VDAG_04769 & VDAG_JR2_Chr6g09500 & $\begin{array}{l}\text { phospho-2-dehydro-3-deoxyheptonate } \\
\text { aldolase }\end{array}$ & + & - \\
\hline 367 VDAG_06253 & VDAG_JR2_Chr7g00300 & cyclic nucleotide-binding protein & + & - \\
\hline 618 VDAG_08151 & VDAG_JR2_Chr8g01560 & hypothetical protein & + & - \\
\hline 48 VDAG_08150 & VDAG_JR2_Chr8g01570 & caib baif family enzyme & - & - \\
\hline
\end{tabular}




\section{T-DNA insertion in intergenic region}

\section{Upstream direction}

\begin{tabular}{|c|c|c|c|c|c|}
\hline Mutant & Dist. $^{\text {a Old locus }}{ }^{b}$ & New locus ${ }^{c}$ & Annotation & Expr. & $\mathrm{CDS}^{\mathrm{e}}$ \\
\hline $459-1$ & 2485 VDAG_00838 & VDAG_JR2_Chr1g01770 & predicted protein & + & - \\
\hline 143 & 374 VDAG_00843 & VDAG_JR2_Chr1g01820 & not annotated & + & - \\
\hline SD-102 & 270 VDAG_00599 & VDAG_JR2_Chr1g20260 & $\begin{array}{l}\text { histidinol-phosphate } \\
\text { aminotransferase }\end{array}$ & + & - \\
\hline 44 & 545 VDAG_08030 & VDAG_JR2_Chr1g22770 & deuterolysin metalloprotease & - & - \\
\hline 11 & 100 VDAG_08003 & VDAG_JR2_Chr1g23050 & f-box protein & - & - \\
\hline SD-107 & 165 VDAG_07796 & VDAG_JR2_Chr1g25310 & hydrolase & + & - \\
\hline 182 & 470 VDAG_09439 & VDAG_JR2_Chr2g01480 & $\begin{array}{l}\text { smc } n \text {-terminal containing } \\
\text { protein }\end{array}$ & + & - \\
\hline 525 & 895 VDAG_02343 & VDAG_JR2_Chr2g01760 & cell wall glucanase & + & 100 \\
\hline SD-7 & 215 VDAG_02014 & VDAG_JR2_Chr2g05250 & myb-like dna-binding protein & + & - \\
\hline 540 & 622 VDAG_01960 & VDAG_JR2_Chr2g05800 & kh protein & + & - \\
\hline 402 & 1788 VDAG_01891 & VDAG_JR2_Chr2g06520 & domain-containing protein & + & 400 \\
\hline 417 & 88 VDAG_01651 & VDAG_JR2_Chr2g09280 & f-box protein & + & - \\
\hline 622 & 700 Not available & VDAG_JR2_Chr3g02050 & unknown & + & - \\
\hline SD-89 & 335 VDAG_07703 & VDAG_JR2_Chr4g00600 & $\begin{array}{l}\text { transcriptional regulatory } \\
\text { protein pro-1 }\end{array}$ & - & - \\
\hline SD-78 & 1214 VDAG_07011 & VDAG_JR2_Chr4g05750 & $\begin{array}{l}\text { subunit of golgi } \\
\text { mannosyltransferase complex }\end{array}$ & + & - \\
\hline 414 & 4 Not available & VDAG_JR2_Chr4g08330 & Myomesin & - & - \\
\hline 584 & 51 VDAG_09007 & VDAG_JR2_Chr4g08540 & family protein & + & - \\
\hline 534 & 737 VDAG_09007 & VDAG_JR2_Chr4g08540 & family protein & + & - \\
\hline 199 & 64 VDAG_05141 & VDAG_JR2_Chr5g01900 & $\begin{array}{l}\text { low-affinity potassium transport } \\
\text { protein }\end{array}$ & - & - \\
\hline 412 & 1647 VDAG_09301 & VDAG_JR2_Chr5g03950 & $\begin{array}{l}\text { potassium sodium efflux } p \text {-type } \\
\text { fungal type }\end{array}$ & + & - \\
\hline 550 & 78 VDAG_04591 & VDAG_JR2_Chr6g07500 & calmodulin & + & - \\
\hline SD-94 & 716 VDAG_04810 & VDAG_JR2_Chr6g09950 & nadph dehydrogenase & + & - \\
\hline 75 & 1060 VDAG_09054 & VDAG_JR2_Chr6g10940 & $\begin{array}{l}\text { ubiquitin fusion degradation } \\
\text { protein }\end{array}$ & + & - \\
\hline 374 & 1023 VDAG_06275 & VDAG_JR2_Chr7g00090 & $\begin{array}{l}\text { maintenance of telomere } \\
\text { capping } 1\end{array}$ & + & \\
\hline 389 & 56 VDAG_06010 & VDAG_JR2_Chr7g02960 & $\begin{array}{l}\text { nad dependent epimerase } \\
\text { dehydratase }\end{array}$ & + & - \\
\hline 438 & 977 VDAG_10534 & VDAG_JR2_Chr7g06650 & ctp synthase & + & - \\
\hline 59 & 47 VDAG_10192 & VDAG_JR2_Chr7g10460 & $\begin{array}{l}\text { benzoate 4-monooxygenase } \\
\text { cytochrome P450 }\end{array}$ & - & - \\
\hline 646 & 246 VDAG 10191 & VDAG_JR2_Chr7g10470 & methyltransferase & + & - \\
\hline 575 & 466 VDAG_03431 & VDAG_JR2_Chr8g09910 & $\begin{array}{l}\text { vegetative cell wall protein } \\
\text { gp1-like }\end{array}$ & - & - \\
\hline
\end{tabular}




\begin{tabular}{|c|c|c|c|c|c|}
\hline \multicolumn{6}{|c|}{ T-DNA insertion in intergenic region } \\
\hline \multicolumn{6}{|c|}{ Downstream direction } \\
\hline Dist. $^{a}$ & Old locus $^{\text {b }}$ & New locus ${ }^{c}$ & Annotation & Expr. & CDS \\
\hline 1784 & VDAG_00839 & VDAG_JR2_Chr1g01780 & $\begin{array}{l}\text { transcription initiation factor tfiid subunit } \\
12\end{array}$ & + & - \\
\hline 100 & VDAG_00844 & VDAG_JR2_Chr1g01830 & exosome complex exonuclease & + & - \\
\hline 746 & VDAG_00600 & VDAG_JR2_Chr1g20270 & acetyl esterase & + & - \\
\hline 669 & VDAG_08031 & VDAG_JR2_Chr1g22760 & saff protein & + & - \\
\hline 3330 & VDAG_08988 & VDAG_JR2_Chr1g23040 & umta methyltransferase & + & - \\
\hline 1586 & VDAG_07797 & VDAG_JR2_Chr1g25300 & briggsae cbr-smu-2 protein & + & - \\
\hline 257 & VDAG_09440 & VDAG_JR2_Chr2g01470 & sgt1-like protein & + & - \\
\hline 71 & VDAG_02344 & VDAG_JR2_Chr2g01750 & $\begin{array}{l}\text { phosphoribosylglycinamide } \\
\text { formyltransferase }\end{array}$ & + & - \\
\hline 1004 & VDAG_02015 & VDAG_JR2_Chr2g05240 & zinc nfx1-type containing protein & - & - \\
\hline 336 & VDAG 01961 & VDAG_JR2_Chr2g05790 & duf974 protein & + & - \\
\hline 1318 & VDAG_01892 & VDAG_JR2_Chr2g06510 & hypothetical protein & + & 100 \\
\hline 731 & VDAG_01652 & VDAG_JR2_Chr2g09270 & cytochrome p450 protein & - & - \\
\hline 101 & VDAG_04427 & VDAG_JR2_Chr3g02040 & agc akt protein kinase & + & - \\
\hline 423 & VDAG 07704 & VDAG_JR2_Chr4g00580 & pathway-specific nitrogen regulator & + & - \\
\hline 1914 & VDAG_07012 & VDAG_JR2_Chr4g05740 & fungal specific transcription factor & + & - \\
\hline 1466 & Not available & VDAG_JR2_Chr4g08340 & umta methyltransferase & - & - \\
\hline 1221 & VDAG_09008 & VDAG_JR2_Chr4g08550 & zinc-binding dehydrogenase & + & - \\
\hline 535 & VDAG_09008 & VDAG_JR2_Chr4g08550 & zinc-binding dehydrogenase & + & - \\
\hline 703 & VDAG_05142 & VDAG_JR2_Chr5g01910 & sodium ion /h ion antiporter & + & - \\
\hline 1459 & VDAG_09302 & VDAG_JR2_Chr5g03940 & glycoside hydrolase family 43 protein & + & 100 \\
\hline 667 & VDAG_04592 & VDAG_JR2_Chr6g07510 & ap-1 complex subunit gamma-1 & + & - \\
\hline 1014 & VDAG_04811 & VDAG_JR2_Chr6g09960 & hypothetical protein & - & 100 \\
\hline 196 & VDAG_09055 & VDAG_JR2_Chr6g10930 & ubiquitin fusion degradation protein & + & - \\
\hline 279 & VDAG_06276 & VDAG_JR2_Chr7g00080 & major facilitator superfamily transporter & + & $\therefore$ \\
\hline 2363 & VDAG_06011 & VDAG_JR2_Chr7g02950 & squalene synthetase & + & - \\
\hline 55077 & VDAG_06595 & VDAG_JR2_Chr7g06640 & sad1 unc protein & + & - \\
\hline 1565 & VDAG_10193 & VDAG_JR2_Chr7g10450 & cellulose-binding family II & + & - \\
\hline 422 & VDAG_10192 & VDAG_JR2_Chr7g10460 & $\begin{array}{l}\text { benzoate 4-monooxygenase } \\
\text { cytochrome p } 450\end{array}$ & - & - \\
\hline 1040 & VDAG_03432 & VDAG_JR2_Chr8g09920 & duf221 protein & + & - \\
\hline
\end{tabular}

a Distance from the T-DNA to the coding sequence (CDS).

${ }^{b}$ Locus corresponds to the V. dahliae strain VdLs.17 assembly deposited on http://genome.jgi.doe. gov/programs/fungi/ (Klosterman et al. 2011).

cLocus corresponds to the V. dahliae strain JR2 assembly deposited on http://fungi.ensembl.org/ (Faino et al. 2015).

dEvidence for in planta expression.

e Evidence for expression in the region between T-DNA and the closest ORF. Distances from T-DNA are indicated in base pairs (bp). 
TABLE 2 | Homologs identified in the PHI database among the 58 potential V. dahliae pathogenicity genes.

\begin{tabular}{|c|c|c|c|c|}
\hline Old locus $^{a}$ & New locus ${ }^{b}$ & Annotation & E-value & Accession $^{c}$ \\
\hline VDAG_00839 & VDAG_JR2_Chr1g01780 & $\begin{array}{l}\text { transcription initiation factor tfiid } \\
\text { subunit } 12\end{array}$ & 7E-19 & PHI:2168 \\
\hline VDAG_00844 & VDAG_JR2_Chr1g01830 & exosome complex exonuclease & $5 \mathrm{E}-12$ & PHI:3472 \\
\hline VDAG_01461 & VDAG_JR2_Chr1g08260 & ring finger protein & $2 \mathrm{E}-09$ & PHI:1393 \\
\hline VDAG_00500 & VDAG_JR2_Chr1g19180 & carboxypeptidase d & $4 \mathrm{E}-55$ & PHI:901 \\
\hline VDAG_09440 & VDAG_JR2_Chr2g01470 & sgt-1-like protein & $1 \mathrm{E}-156$ & PHI: 1615 \\
\hline VDAG_09439 & VDAG_JR2_Chr2g01480 & smc n-terminal containing protein & $2 \mathrm{E}-10$ & PHI:359 \\
\hline VDAG_02343 & VDAG_JR2_Chr2g01760 & cell wall glucanase & $9 E-30$ & PHI:816 \\
\hline VDAG_02014 & VDAG_JR2_Chr2g05250 & myb-like dna-binding protein & $1 \mathrm{E}-105$ & PHI:1540 \\
\hline VDAG_01960 & VDAG_JR2_Chr2g05800 & kh protein & $3 E-22$ & PHI:1662 \\
\hline VDAG_04427 & VDAG_JR2_Chr3g02040 & agc akt protein kinase & $0 E+00$ & PHI:3131 \\
\hline VDAG_07704 & VDAG_JR2_Chr4g00580 & pathway-specific nitrogen regulator & $0 E+00$ & PHI:1960 \\
\hline VDAG_07012 & VDAG_JR2_Chr4g05740 & fungal specific transcription factor & $0 E+00$ & PHI:1954 \\
\hline VDAG_09008 & VDAG_JR2_Chr4g08550 & zinc-binding dehydrogenase & $7 E-13$ & $\mathrm{PHI}: 2895$ \\
\hline VDAG_07314 & VDAG_JR2_Chr4g10770 & copper amine oxidase 1 & $1 \mathrm{E}-106$ & PHI:2171 \\
\hline VDAG_09301 & VDAG_JR2_Chr5g03950 & $\begin{array}{l}\text { potassium sodium efflux p-type } \\
\text { fungal type }\end{array}$ & $0 \mathrm{E}+00$ & PHI:2098 \\
\hline VDAG_04591 & VDAG_JR2_Chr6g07500 & calmodulin & $4 \mathrm{E}-20$ & $\mathrm{PHI}: 2640$ \\
\hline VDAG_06253 & VDAG_JR2_Chr7g00300 & cyclic nucleotide-binding protein & $2 \mathrm{E}-10$ & PHI:372 \\
\hline VDAG_10193 & VDAG_JR2_Chr7g10450 & cellulose-binding family II & $6 \mathrm{E}-13$ & $\mathrm{PHI}: 2388$ \\
\hline VDAG_10191 & VDAG_JR2_Chr7g10470 & methyltransferase & $9 \mathrm{E}-40$ & PHI:2315 \\
\hline
\end{tabular}

a Locus corresponds to the V. dahliae strain VdLs.17 assembly deposited on http://genome.jgi.doe.gov/ programs/fungi/ (Klosterman et al. 2011).

${ }^{b}$ Locus corresponds to the $\mathrm{V}$. dahliae strain JR2 assembly deposited on http://fungi.ensembl.org/_Faino et al. 2015).

cPathogen-host interaction database accession.

\section{Analysis of a T-DNA insertion site links VdNRS/ER to virulence}

In random mutant 389, the T-DNA was integrated 56 bp upstream of the coding region of a nucleotide-rhamnose synthase/epimerase-reductase (NRS/ER) homolog VDAG_06010 (Table 1; (Klosterman et al. 2011)), which corresponds to VDAG_JR2_ Chr7g02960 in the V. dahliae JR2 assembly deposited at http://fungi.ensembl. org/ (Faino et al. 2015). The first CDS downstream of the T-DNA insertion site is found only at $2.4 \mathrm{~kb}$, encoding a squalene synthetase (VDAG_06011 or VDAG_ JR2_Chr7g02950), an enzyme that has been implicated in sterol and triterpene biosynthesis. However, considering the distance between the T-DNA insertion and the CDS of this gene it is unlikely that it is affected and causal to the reduced pathogenicity phenotype of the RM-389 mutant. To assess whether any genes have been overlooked by the automated gene prediction in the region between the 
T-DNA insertion and the squalene synthetase CDS, RNAseq reads from V. dahliaeinoculated N. benthamiana (de Jonge et al. 2012; Faino et al. 2012) were queried. However, no reads were found to map to this region suggesting that no in planta transcribed genes reside in this region. Finally, with real-time PCR on genomic DNA, using the single copy Ave1 gene as a reference (de Jonge et al. 2012), it was determined that only a single T-DNA insertion was present in the genome of the RM-389 mutant.

V. dahliae NRS/ER (VdNRS/ER) shows weak (17\%) amino-acid sequence similarity to the Salmonella enterica dTDP-rhamnose biosynthesis component rmID, a bacterial protein involved in the last step in the conversion of dTDP-glucose to dTDPrhamnose. In bacteria the nucleotide sugar dTDP-rhamnose is the precursor that facilitates the incorporation of L-rhamnose into glycan, which has been implicated in virulence of pathogenic bacteria such as Salmonella enterica, Vibrio cholerae and Streptococcus mutans (Giraud et al. 2000). Thus, considering all of the above, functional analysis of the VdNRS/ER gene was pursued.

\section{VdNRS/ER is required for V. dahliae pathogenicity}

Targeted replacement of VdNRS/ER by a hygromycin resistance cassette through homologous recombination was pursued in wild-type $V$. dahliae. Several independent VdNRS/ER deletion strains were obtained, of which two ( $\triangle 6010-1$ and $\triangle 6010-2)$ were used for further analysis (Supplemental Figure 1). To assess the role of VdNRS/ER in $V$. dahliae vegetative growth and conidiospore production, radial growth and sporulation of the VdNRS/ER deletion strains on potato dextrose agar was assessed together with the random mutant (RM-389) and an ectopic transformant with a random T-DNA insertion outside of the VdNRS/ER gene. This analysis showed that growth and conidiation was not markedly affected upon VdNRS/ER deletion (Figure 2).

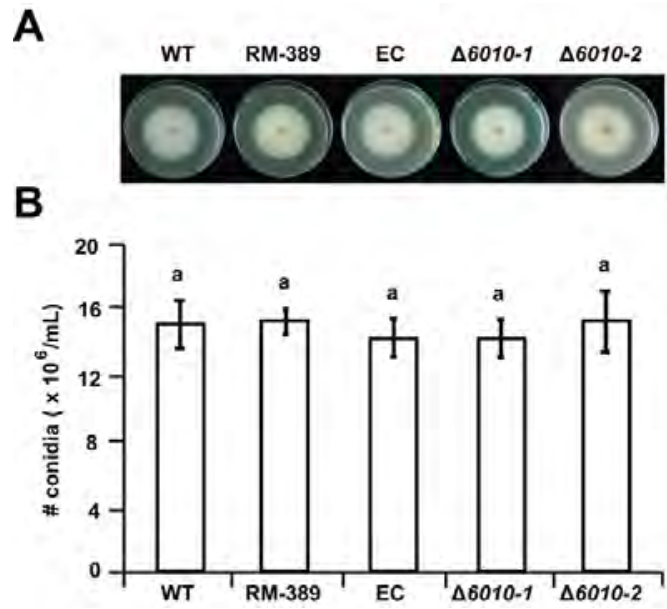

FIGURE 2 | Targeted deletion of VdNRS/ER does not impair growth and conidiogenesis. (A) Radial growth and colony morphology of wild type $V$. dahliae (WT), random transformant RM-389, an ectopic transformant (EC) and two VdNRS/ $E R$ deletion strains $(\triangle 6010-1$ and $\triangle 6010-2)$ after 7 days of incubation on PDA medium at $22^{\circ} \mathrm{C}$. (B) Average number of conidia produced after 7 days of growth on PDA medium based on two independent experiments. Letters indicate significant differences $(P<0.05)$ calculated with the Student's t test. 
Subsequently, the role of VdNRS/ER in V. dahliae pathogenicity was addressed. To this end, the expression of VdNRS/ER was assessed in wild-type $V$. dahliae during infection of tomato plants in a time course harvested at regular intervals after inoculation, showing that VdNRS/ER is induced during in planta growth with a peak in expression at around 12 days post inoculation (Figure 3). Subsequently, the VdNRS/ER deletion strains were inoculated on tomato plants alongside the random mutant (RM-389) and an ectopic transformant to investigate the role of VdNRS/ $E R$ in fungal virulence. While inoculation of the plants with the wild-type $V$. dahliae strain and the ectopic transformant resulted in the development of symptoms of Verticillium wilt disease that include stunting of the plants and wilting, inoculation with RM-398 or the VdNRS/ER deletion strains did not result in disease development (Figure 4A). The absence of disease symptoms was confirmed by measuring the surface area of the foliage of the plants that were inoculated with the various fungal strains. Plants that were inoculated with VdNRS/ER deletion strains developed similar foliage (canopy) areas as mock-inoculated plants and plants inoculated with RM-389 (Figure 5A). In contrast, less foliage developed for plants that were inoculated with the wild-type $V$. dahliae strain and the ectopic transformant (Figure $5 A)$. Thus, we conclude that $V d N R S / E R$ is crucial for disease development by $V$. dahliae. To assess whether VdNRS/ER deletion strains retained the capability to colonize tomato plants, fungal outgrowth assays were performed by plating of stem sections harvested from the hypocotyls of the inoculated plants. Interestingly, while the ectopic transformants and the wild-type strain had extensively colonized the stems of the tomato plants, no outgrowth was observed for the VdNRS/ER deletion strains and RM-389, demonstrating that these genotypes were not able to colonize the xylem vessels of the inoculated tomato plants (Figure 4A). The absence of tomato colonization by VdNRS/ER deletion strains was confirmed with real-time PCR quantification of fungal biomass (Figure 5B). Importantly, pathogenicity was restored upon complementation of the VdNRS/ER deletion strains with a genomic construct containing the wild-type VdNRS/ER locus (Supplemental Figure 2). Thus, it can be concluded that $V d N R S / E R$ is required for pathogenicity on tomato.

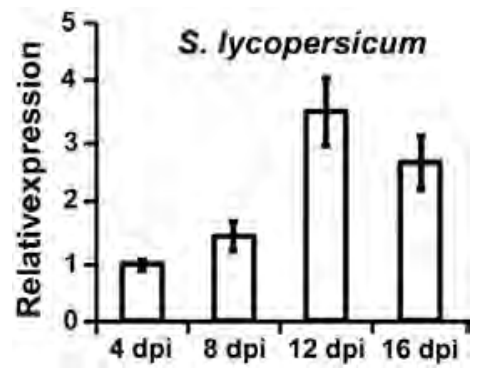

FIGURE 3 | Expression of VdNRS/ER during infection of Verticillium dahliae on tomato. Ten-day-old tomato (S. lycopersicum) cultivar MoneyMaker plants were rootinoculated with $V$. dahliae and plants were harvested at regular intervals from 4 to 16 days post inoculation (dpi). After RNA isolation and cDNA synthesis, real-time PCR was performed to determine the relative expression levels of $V d N R S / E R$ using the $V$. dahliae elongation factor 1 -alpha gene as a reference. Expression at $4 \mathrm{dpi}$ is set to one for all panels. 
To investigate whether the observed role of VdNRS/ER in pathogenicity is confined to tomato only, or also extends to other host species, we tested the pathogenicity of the VdNRS/ER deletion strains on the Solanaceous model plant Nicotiana benthamiana. Similar to tomato, targeted deletion of VdNRS/ER resulted in compromised pathogenicity, as also these plants remained devoid of Verticillium wilt symptoms (Figure 4B). Again, loss of pathogenicity was confirmed by measurement of the canopy surface area (Figure $5 \mathrm{C}$ ), and loss of pathogenicity was confirmed by real-time PCR quantification of fungal biomass (Figure 5D).

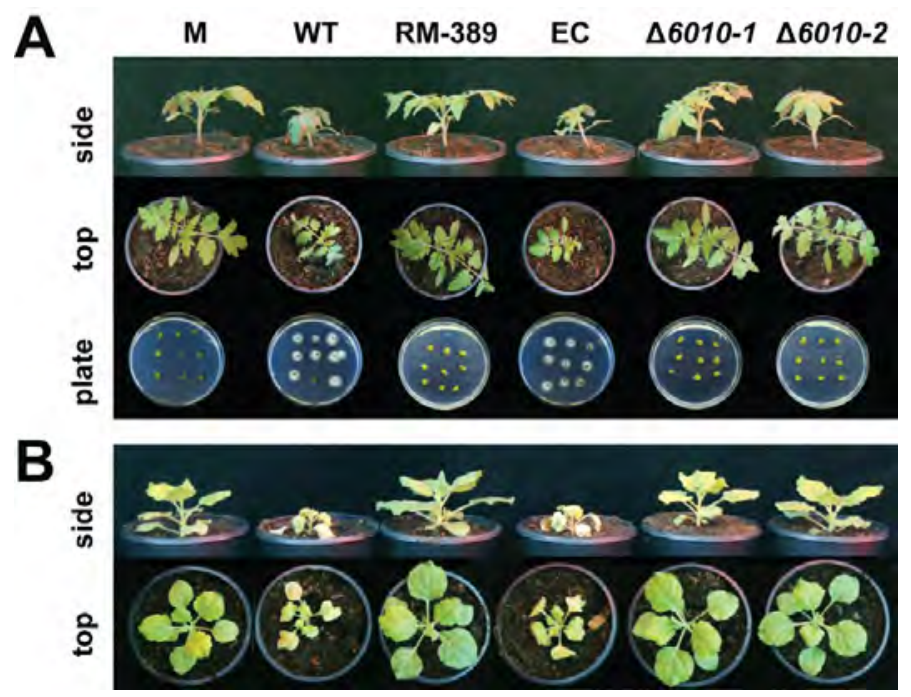

FIGURE 4 | VdNRS/ER is required for pathogenicity of Verticillium dahliae on tomato and Nicotiana benthamiana. (A) Top and side view of tomato cultivar MoneyMaker plants that were mock-inoculated (M), or inoculated with wild type V. dahliae (WT), random transformant RM-389, an ectopic transformant (EC), and two VdNRS/ER deletion strains ( $\triangle 6010-1$ and $\triangle 6010-2)$ at $14 \mathrm{dpi}$. Fungal outgrowth at 7 days after plating of stem sections harvested at 14 days post inoculation is shown at the bottom of the panel. (B) Top and side view of $N$. benthamiana plants inoculated as specified for panel A.

\section{Absence of VdNRS/ER variation in a collection of $V$. dahliae strains}

Allelic variation can be a sign of selection pressure and adaptation. To analyze potential VdNRS/ER diversity, we mined the genomes of 39 sequenced $V$. dahliae strains for the VdNRS/ER gene sequence (de Jonge et al. 2012) (Faino and Thomma, unpublished data). Intruigingly, only two single nucleotide polymorphisms (SNPs) were identified in two strains, each at a different position and both resulting in a synonymous substitution that does not affect the sequence of the encoded protein. We thus conclude that these substitutions do not affect the function of VdNRS/ER. 

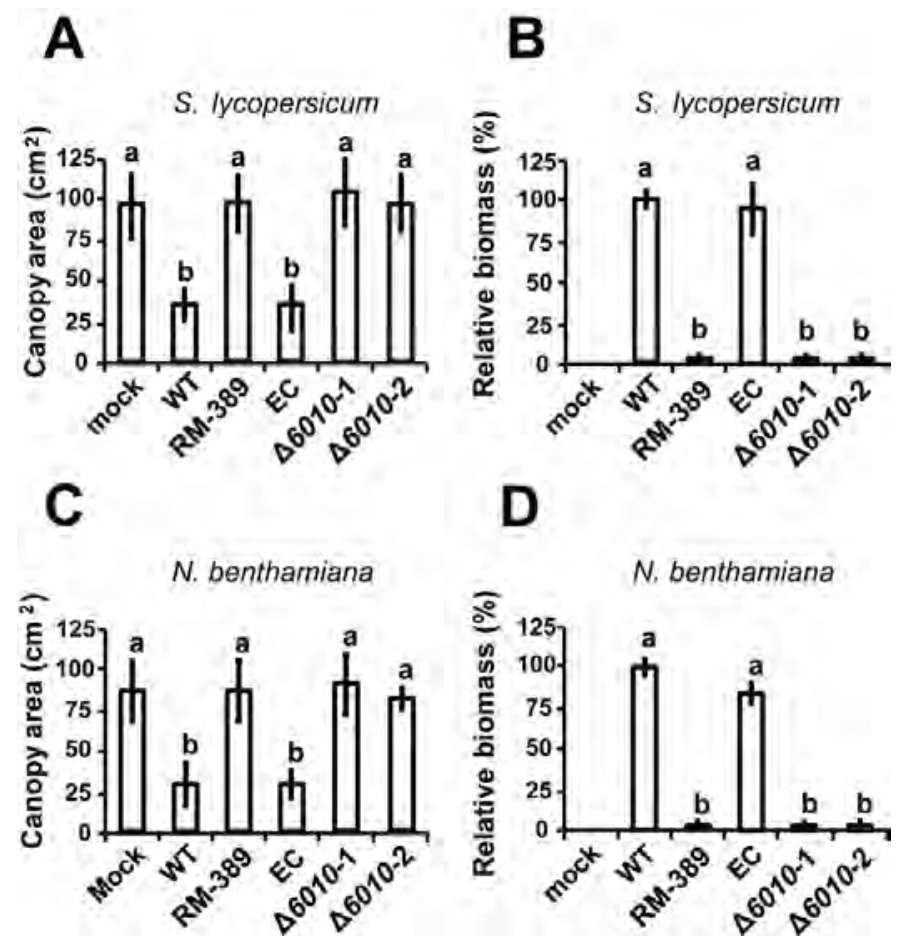

FIGURE 5 | VdNRS/ER is required for pathogenicity of Verticillium dahliae on tomato and Nicotiana benthamiana. (A) Average canopy area of 6 tomato plants, and (B) real-time PCR quantification of fungal biomass at 14 days after mock-inoculation (mock), or inoculation with wild type Verticillium dahliae (WT), random transformant (RM-389), an ectopic transformant (EC), and two VdNRS/ER deletion strains $(\triangle 6010-1$ and $\triangle 6010-2)$. Different letter labels indicate significant differences $(P<0.05)$. (C) Average canopy area of $6 \mathrm{~N}$. benthamiana plants, and $(D)$ real-time $P C R$ quantification of fungal biomass upon inoculation as specified for panels $A$ and $B$.

\section{VdNRS/ER is not required for cell wall integrity and osmotic stress resistance}

To examine the role of VdNRS/ER in cell wall integrity and osmotic stress resistance, the random mutant (RM-389), the VdNRS/ER deletion strains and the ectopic transformant were grown on minimal agar medium supplemented withmannitol, sodium chloride, sorbitol or congo red. After seven days of growth the colony diameter was measured, showing that mutant and wild type strains were equally sensitive to the cell wall stress reagents (Figure 6). These findings suggest that $V d N R S / E R$ is not involved in cell wall integrity or osmotic stress resistance during mycelium development. 

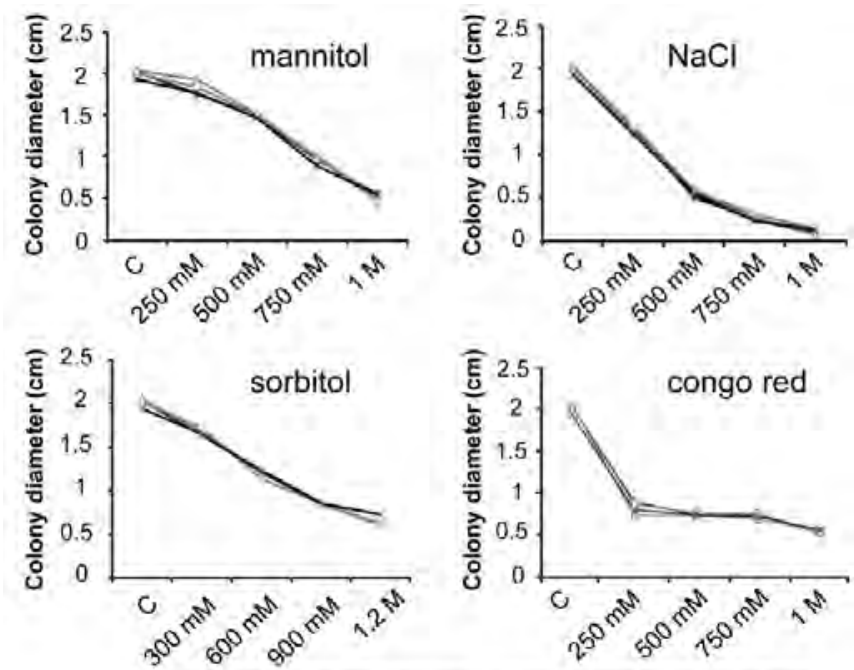

$\diamond-W T \rightarrow \square-\Delta 6010-1 \rightarrow \Delta-\Delta 6010-2 \rightarrow-E C$

FIGURE 6 | Targeted deletion of VdNRS/ER does not affect cell wall integrity and osmotic stress resistance. Stress sensitivity assays were performed by placing a $5 \mu \mathrm{L}$ droplet $\left(10^{6}\right.$ conidia/ $\mathrm{mL}$ ) of wild type $V$. dahliae (WT), an ectopic transformant (EC) and two VdNRS/ER deletion strains ( $\triangle 6010-1$ and $\triangle 6010-2)$ on Czapek-dox medium (C), or Czapek-dox supplemented with congo red $(250 \mathrm{mM}, 500 \mathrm{mM}, 750 \mathrm{mM}$ and $1 \mathrm{M}), \mathrm{NaCl}(250 \mathrm{mM}, 500 \mathrm{mM}, 750 \mathrm{mM}$ and $1 \mathrm{M})$, mannitol (250 mM, $500 \mathrm{mM}, 750 \mathrm{mM}$ and $1 \mathrm{M}$ ), or sorbitol (300 mM, $600 \mathrm{mM}, 900 \mathrm{mM}, 1.2 \mathrm{M})$. The colony diameter was measured after 7 days of incubation at $22^{\circ} \mathrm{C}$.

\section{VdNRS/ER is required for tomato root colonization}

To examine the role of $V d N R S / E R$ in the initial stages of $V$. dahliae infection, roots of ten-day-old tomato plants grown in a hydroponics solution were inoculated with conidiospores of the random mutant (RM-389), the VdNRS/ER deletion strains and the ectopic transformant. After five days of incubation, the tomato roots were inspected using a binocular microscope. Our data show that wild type spores efficiently attached to the root surface, resulting in extensive fungal growth on the tomato roots (Figure 7A). In contrast, little fungal growth was observed on roots inoculated with the random mutant (RM-389) or the VdNRS/ER deletion strains (Figure 7A). The reduced fungal biomass of the random mutant (RM-389) and the VdNRS/ER deletion strains on the tomato roots was confirmed by quantitative PCR (Figure 7B). Collectively, these data show that VdNRS/ER functions to contribute to successful colonization of roots by $V$. dahliae, potentially through root attachment. 

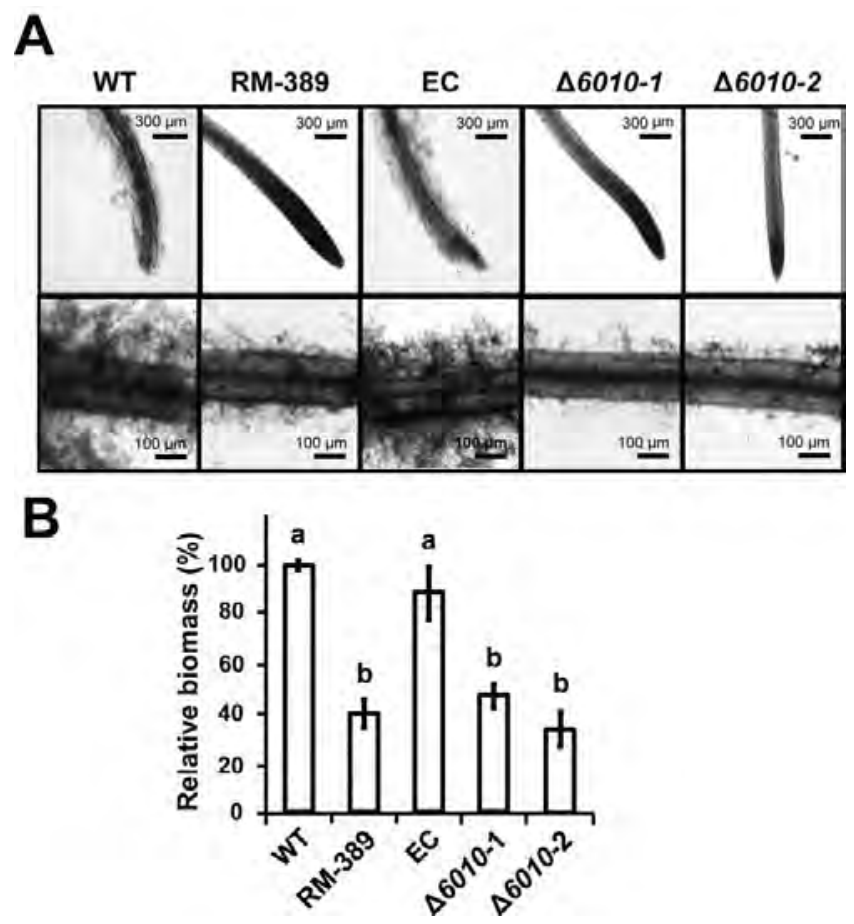

FIGURE $7 \mid$ VdNRS/ER is required for colonization of tomato roots. Roots of ten-day-old tomato cultivar MoneyMaker seedlings were immersed in one-fifth PDB containing $10^{6}$ conidia/mL of wild type $V$. dahliae (WT), random transformant (RM-389), an ectopic transformant (EC) and two VdNRS/ER deletion strains ( $\triangle 6010-1$ and $\triangle 6010-2)$ for $72-96 \mathrm{~h}$. A) Roots were rinsed with water and photographed under a microscope. B) Real-time PCR quantification of fungal biomass on the roots. Different letter labels indicate significant differences $(P<0.05)$.

\section{VdNRS/ER is a functional enzyme that converts UDP-KDG to UDP-rhamnose}

To examine whether VdNRS/ER is involved in the biosynthesis of UDP-rhamnose and determine whether this activated sugar is the precursor for the synthesis of rhamnose-containing glycan, we examined the polysaccharide composition of $V$. dahliae. Polysaccharides were extracted from conidiospores of wild type V. dahliae, the VdNRS/ER deletion strain and the complementation strain, and analysed by GC-MS. In the samples derived from wild type $V$. dahliae and the complementation strain a peak was detected that corresponds to rhamnose, which was absent in the $V d N R S / E R$ deletion strain (Figure 8). This finding confirms that VdNRS/ER is involved in rhamnose biosynthesis. 


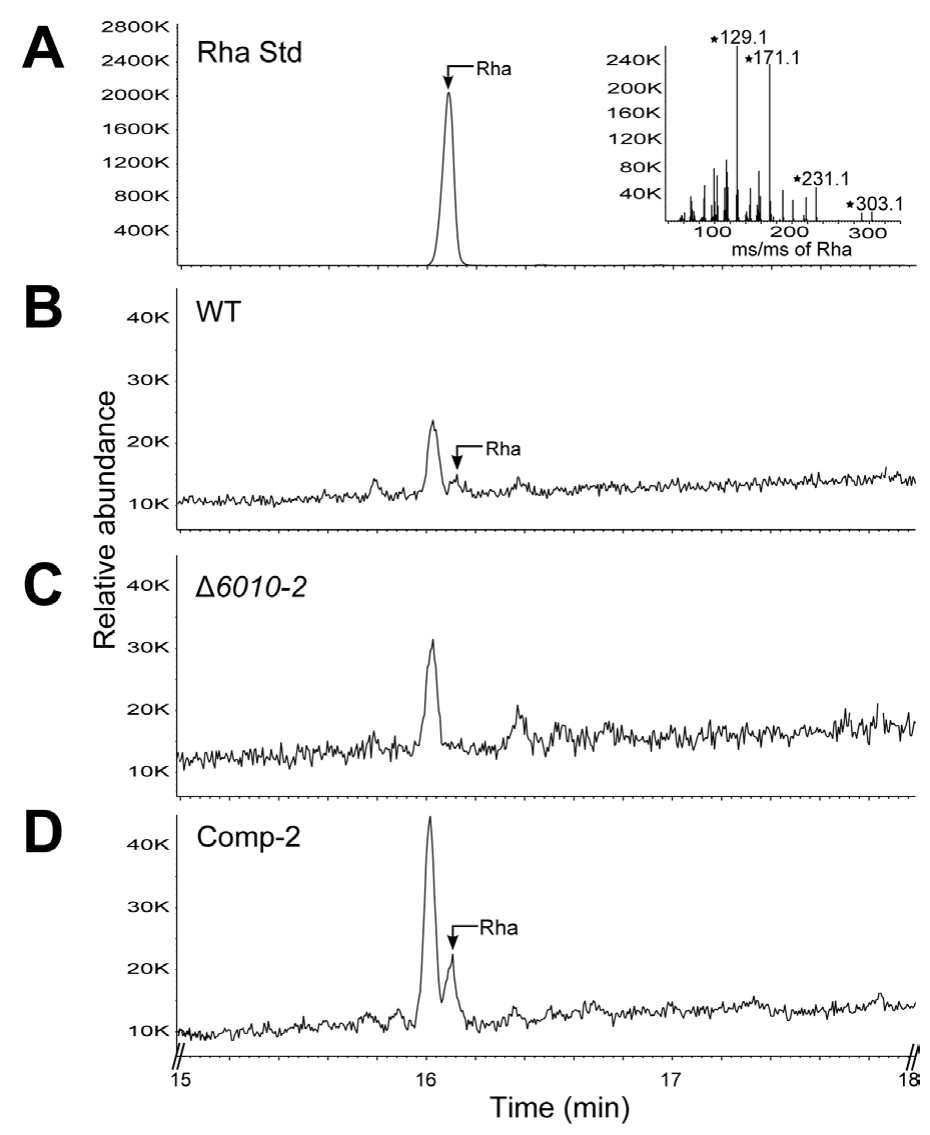

FIGURE 8 | VdNRS/ER deletion strains are depleted in UDP-rhamnose. GC-MS analysis of alditol acetate derivatives of the glycosyl residues from conidiospore polysaccharides (A) Total ion count chromatogram of alditol-acetate derived standard rhamnose (Rha), and mass spectra (insert) showing fragmentation pattern fingerprints with $\mathrm{m} / \mathrm{z} 129,171,231,303$. (B) Polysaccharides from wild type $V$. dahliae (WT) conidiospores display a peak eluting at 16.10 minutes that has the same retention time as rhamnose standard. (C) VdNRS/ER deletion strain ( $\triangle 6010-1)$ lacks a peak eluting at 16.10 minutes. (D) VdNRS/ER deletion strain complemented with native VdNRS/ER gene (Comp-2), displays a peak eluting at 16.10 minutes that has the same retention time as the rhamnose standard.

If rhamnose-containing glycans play a role during early stages of host colonization, such as attachment of conidiospores to the root, we anticipated that such glycans are produced during conidiospore germination. Therefore, we determined the sugar composition of germinating conidiospores. Indeed, the sample derived from wild type V. dahliae comprised a clear rhamnose peak (Figure 9A;B) that was absent in samples from the VdNRS/ER deletion strain (Figure 9C). Taken together, VdNRS/ $E R$ plays a role in the formation of rhamnose-containing glycan in germinating conidiospores during early stages of host colonization. 


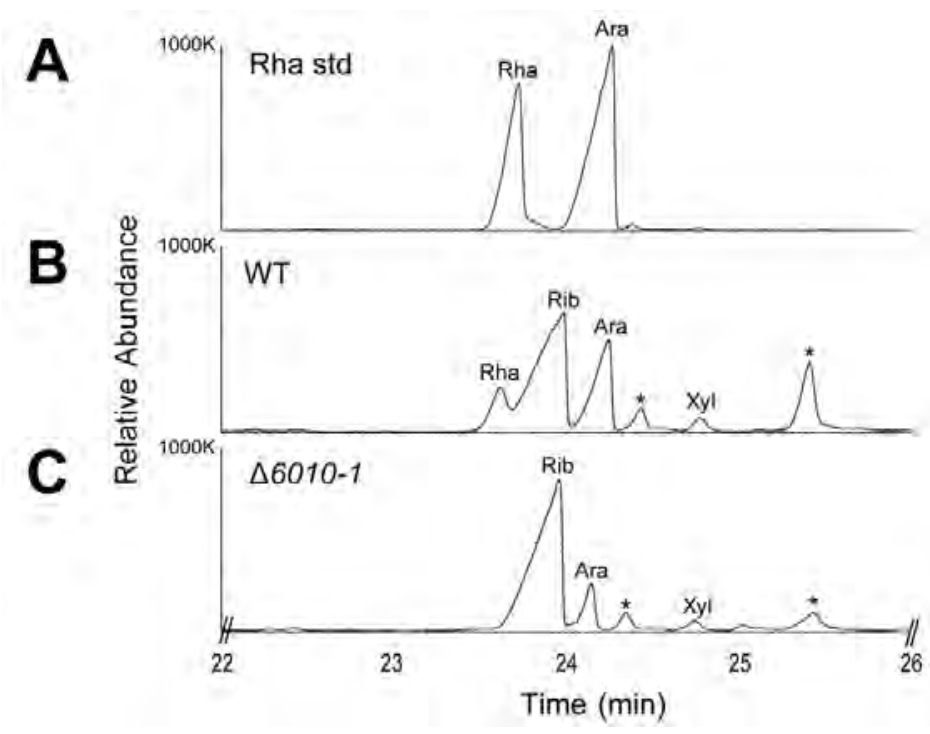

FIGURE 9 | UDP-rhamnose is produced in germinating conidiospores. GC-MS analysis of alditol acetate derivatives of the glycosyl residues from germinating conidiospore polysaccharides (A) Total ion count chromatogram of alditol-acetate derived standard rhamnose (Rha) (B) Polysaccharides of conidiospores from wild type $V$. dahliae (WT) display a peak with the same retention time as the rhamnose standard. (C) VdNRS/ER deletion strain $(\triangle 6010-1)$ does not display a rhamnose peak. Other peaks in the chromatogram correspond to ribose (rib), arabinose (ara) and xylose (xyl). Asterisks indicate unidentified compounds that are not sugars.

In contrast to bacteria that produce dTDP-rhamnose in a three-step pathway, plants follow a two-step pathway to synthesize UDP-rhamnose (Oka et al. 2007; Watt et al. 2004). Genes involved in UDP-rhamnose were recently also identified in other fungi, including the rice pathogen Magnaporthe oryzae and the broad host range pathogen Botrytis cinerea (Martinez et al. 2012). In B. cinerea, UDP-glucose is converted by a 4,6-dehydratase (BfDh) into an intermediate UDP-KDG (UDP-4-keto-6-deoxyglucose) that is subsequently converted by a bifunctional 3,5-epimerase/4-reductase (BfER, i.e. NRS/ER) into UDP-rhamnose (Martinez et al. 2012). Thus, we examined whether VdNRS/ER, like BfER, is a functional enzyme that can convert UDP-KDG to UDP-rhamnose. To this end, the dual enzyme activities of recombinant VdNRS/ER and BfDh was analyzed by LC-MS using UDP-glucose as a substrate (Martinez et al. 2012). The first enzyme in the pathway is capable to convert UDP-Glc to UDP-KDG (Figure 10B, and D) as expected, and with the addition of the NRS/ER activity (VdNRS/ER) UDP-KDG was converted to UDP-rhamnose (Figure 10C). The authenticity of UDPrhamnose was determined by its elution and by its mass and $\mathrm{ms} / \mathrm{ms}$ ion fragmentation (see insert in Fig 10C). The enzyme activity of recombinant VdNRS/ER unambiguously shows that VdNRS/ER is a bifunctional enzyme with a 3,5-epimerase and 4-reductase 
activities (Figure 10E). Thus, we conclude that deletion of VdNRS/ER contributed to the loss of UDP-rhamnose formation and the lack of rhamnose-containing glycans during spore germination and early stages of infection.
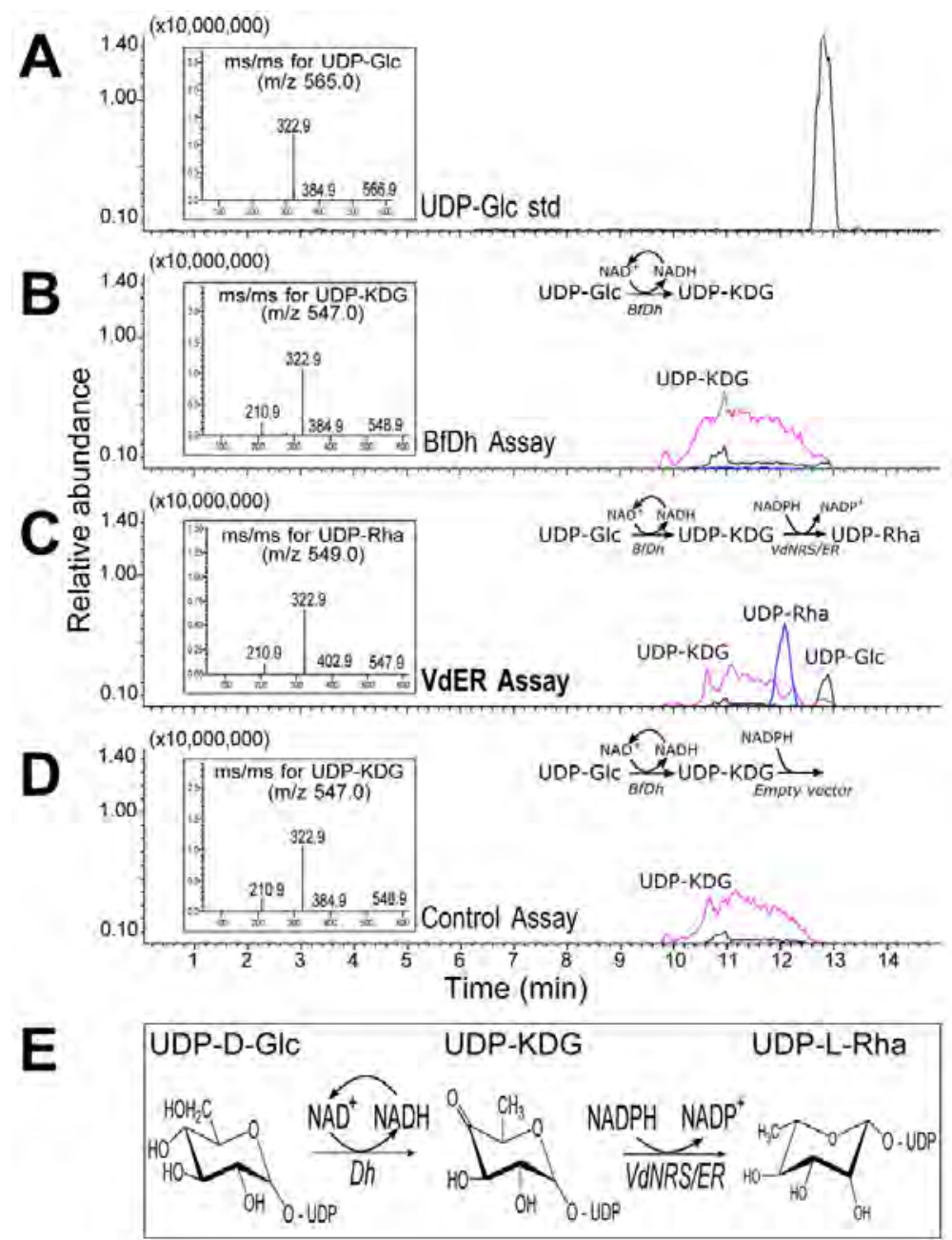

FIGURE 10 | VdNRS/ER converts UDP-KDG to UDP-rhamnose. (A) UDP-glucose (UDP-Glc) standard elutes from Hilic column at 12.8 minutes and is detected by MS with $\mathrm{m} / \mathrm{z} 565[\mathrm{M}-\mathrm{H}]$. The $\mathrm{ms} / \mathrm{ms}$ of the parent ion (insert) gave $\mathrm{m} / \mathrm{z} 323$ and 385 diagnostic ion fragments [UMP-H] and [UDP-H2O-H]; respectively. (B) Hilic separation of products from the enzymatic reaction with $\mathrm{BfDh}$ displays a peak with the same rentention time as UDP-4-keto-6-deoxyglucose (UDP-KDG) with diagnostic [M-H] $\mathrm{m} / \mathrm{z} 547$ and $\mathrm{ms} / \mathrm{ms}$ ion fragment with $\mathrm{m} / \mathrm{z}$ 323. (C) Hilic separation of of products from the dual enzymatic reaction with BfDh and VdNRS/ER displays a peak with the same rentention time as UDP-rhamnose (UDP-Rha) with diagnostic [M-H] $\mathrm{m} / \mathrm{z} \mathrm{549}$, and $\mathrm{ms} / \mathrm{ms}$ of 403, 323,210 . (D) Dual enzymatic reaction with $B f D h$ and an empty vector control displays a peak with the same rentention time as UDP-4-keto-6-deoxyglucose (UDP-KDG) with diagnostic [M-H]- $\mathrm{m} / \mathrm{z}$ 547 and $\mathrm{ms} / \mathrm{ms}$ ion fragment with $\mathrm{m} / \mathrm{z}$ 323. (E) UDP-rhamnose metabolic pathway in fungi showing the enzymes involved in the sequential conversion of UDP-Glc to UDP-Rha. 


\section{Discussion}

In our study on a random mutant library that was generated in the vascular wilt fungus V. dahliae, we show for the first time that rhamnose containing macromolecules are required for fungal pathogenicity through their contribution to colonization of tomato roots. In random mutant 389, the T-DNA was integrated 56 bp upstream of the coding region of a putative nucleotide-rhamnose synthase/epimerasereductase (NRS/ER). Our biochemical analyses of cell wall polysaccharides (Figures 8 and 9) and enzyme activity (Figure 10C) confirmed that VdNRS/ER is involved in UDP-rhamnose biosynthesis and shows that this activated sugar is the precursor for rhamnose-containing glycan.

VdNRS/ER shows similarity to the bacterial rmID substrate binding domain, which is involved in biosynthesis of dTDP-rhamnose. In Gram-negative bacteria such as $S$. enterica, V. cholerae or Escherichia coli, L-rhamnose is an important residue in the O-antigen of lipopolysaccharides, which are essential for resistance to serum killing and intestinal colonization (Chiang and Mekalanos 1999). In mycobacteria such as Mycobacterium tuberculosis, L-rhamnose maintains the structural integrity of the cell wall through connecting the inner peptidoglycan layer to the arabinogalactan polysaccharides (Giraud et al. 2000). Moreover, disruption of dTDP-rhamnose biosynthesis in the plant growth-promoting rhizobacteria Azospirillum brasilense and Azorhizobium caulinodans resulted in reduced root attachment and colonization (Gao et al. 2001; Jofré et al. 2004). Work on Colletotrichum graminicola, causal agent of stalk rot of cereals and anthracnose of maize revealed the secretion of mucilaginous matrix by the fungus to promote survival of fungal spores, the major component of which appeared to be high molecular weight glycoproteins containing rhamnose and mannose oligomers (Ramadoss et al. 1985). Subsequently, this matrix was proposed to allow for the rapid adhesion of even ungerminated conidia to the hydrophobic plant surface to ensure germination and appressorium formation and establishment of an infection court (Nicholson and Kunoh 1995). Interestingly, further rhamnose-containing extracellular matrix is released upon germ tube elongation to surround and extend between hyphae (Sugui et al. 1999). Finally, a recent study identified two genes encoding UG4,6-Dh and U4k6dG-ER enzymes from Magnaporthe oryzae and Botrytis cinerea involved in UDP-rhamnose formation, although their contribution to pathogenicity was not investigated (Martinez et al. 2012). Based on our study, however, strong evidence is presented that fungal UG4,6-Dh and U4k6dG-ER enzymes are involved in the formation of UDP-rhamnose that is incorporated into rhamnose-containing polysaccharides that are crucial for fungal pathogenicity. Here, we have shown that targeted deletion of $V d N R S / E R$ contributes to loss of $V$. dahliae pathogenicity on various host plants. 
The role of rhamnose-containing macromolecules in cell wall integrity and oxidative stress tolerance in fungi is largely unknown. VdNRS/ER deletion strains were able to grow and sporulate without any visible defects, suggesting that rhamnosecontaining glycans such as glycoproteins, polysaccharides or exopolysacharides, are not essential for vegetative growth or sporulation. In addition, the growth rate of $V d N R S / E R$ knock-out mutants was not affected in the presence of inducers of cell wall stress and osmotic stress, suggesting that the rhamnose containing glycans are not involved in functional cell wall structure and osmotic stress tolerance.

Based on the findings reported in this study, we propose a model to explain the contribution of UDP-rhamnose to the virulence of $V$. dahliae during infection on host plants. In the cytoplasm UDP-glucose is converted into UDP-rhamnose in two steps using VdUG4,6-Dh and VdNRS/ER. The rhamnose of UDP-rhamnose is then transferred to glycan(s). The nature of these glycans remains unknown, but they consist of $\mathrm{NaOH}$-solubilized cell wall polysaccharides and potentially glycoproteins. Presently, we cannot exclude the possibility that the rhamnosecontaining polysaccharide is not an extracellular polysaccharide (EPS) as shown to be the case in Botrytis cinerea. We propose that the rhamnose-containing glycan, produced during spore germination, plays a role in the attachment of spores to the root surface which results in successful colonization of host plants. As VdNRS/ $E R$ deletion strains are unable to synthesize UDP-rhamnose and unable to cause disease, inhibitors of UDP-rhamnose synthesis may result in impaired pathogenicity and could present a novel strategy for disease control.

\section{Materials And Methods}

\section{Agrobacterium tumefaciens mediated transformation (ATMT)}

The binary vector ( $\mathrm{pBH}+2)$ harboring the hygromycin B resistance gene (hph) under control of the Aspergillus nidulans trpC promoter was used for ATMT (Mullins et al. 2001). This vector was introduced to Agrobacterium tumefaciens strain SK1044 to transform conidia of race $1 \mathrm{~V}$. dahliae strain JR2 as described previously (Faino et al. 2015; Santhanam 2012). Briefly, A. tumefaciens was grown at $28^{\circ} \mathrm{C}$ for 2 days in minimal medium supplemented with kanamycin $(25 \mu \mathrm{g} / \mathrm{mL})$. The A. tumefaciens cells were diluted to an $\mathrm{OD}_{600}$ of 0.15 in induction medium (IM), supplemented with $200 \mu \mathrm{M}$ acetosyringone (AS). The cells were grown for an additional six hours before mixing them with an equal volume of $V$. dahliae conidiospore suspension ( $10^{6}$ conidia/mL). $200 \mu \mathrm{l}$ of this mixture was plated onto a Hybond- $\mathrm{N}^{+}$filter placed on induction medium supplemented with $200 \mu \mathrm{M}$ acetosyringone. The plates were incubated in the dark at room temperature for $48 \mathrm{~h}$ after which the filter was 
transferred onto a selection plate (PDA supplemented with $50 \mu \mathrm{g} / \mathrm{mL}$ of hygromycin $\mathrm{B}$ and $200 \mu \mathrm{M}$ of cefotaxime). After 10 to 14 days, individual transformants were transferred to 24-well culture plates containing one $\mathrm{mL}$ of selection medium and incubated for 7 to 10 days. Spores from these cultures were stored in $30 \%$ glycerol at $-80^{\circ} \mathrm{C}$ until further analysis.

\section{Plant inoculations}

Pathogenicity assays were performed as described previously (Santhanam and Thomma 2013). Briefly, individual transformants were sub-cultured in six-welled culture plates for 7 to 10 days. Subsequently, 10 glass beads $(\sim 3 \mathrm{~mm})$ and $3 \mathrm{~mL}$ of tap water were added to each of the wells and the plates were sealed. The conidiospores were released by shaking the plates for $15 \mathrm{~min}$ at $200 \mathrm{rpm}$ on a reciprocal shaker after which the sealing was removed. The roots of 10-day-old tomato (Solanum lycopersicum cv. MoneyMaker) seedlings were rinsed in water and dipped into the conidiospore suspension for 5 minutes. Seedlings were replanted in soil and scored for symptom development (wilting and stunting) up to 14 days. Seedlings that exhibited reduced Verticillium wilt symptoms when compared to inoculation with wild type $V$. dahliae inoculated plants were identified and the corresponding mutants were retained for rescreening.

During rescreening, the infection assay was carried out essentially as described above, with the modification that the conidiospore concentration of the transformants was calibrated to $10^{6}$ conidiospores $/ \mathrm{mL}$. The rescreening was repeated at least two times for each of the mutants that were retained in the initial screen.

\section{Identification of T-DNA insertion sites}

Genomic sequences flanking the T-DNA were isolated from the selected transformants with inverse PCR (iPCR) as described previously (Meng et al. 2007; Santhanam 2012). Essentially, 500 ng of genomic DNA was digested overnight with $\mathrm{Mspl}$ or $\mathrm{Ncol}$ and heat-inactivated at $65^{\circ} \mathrm{C}$ for $20 \mathrm{~min}$. Subsequently, $50 \mu \mathrm{L}$ of ligation mix ( $10 \mu \mathrm{L}$ of 10X T4 DNA ligase buffer, 5 units of T4 DNA ligase and $38 \mu \mathrm{L}$ of $\mathrm{H}_{2} \mathrm{O}$ ) was added and incubated overnight at $15^{\circ} \mathrm{C}$. Next, the DNA was precipitated and dissolved in $50 \mu \mathrm{L}$ of demineralized water. The genomic region flanking the T-DNA was amplified in $50 \mu \mathrm{L}$ reaction mix using $2 \mu \mathrm{L}$ of ligation product, $1 \mu \mathrm{L}$ of each primer (Supplemental Table 1), 1X PCR buffer, $0.6 \mu \mathrm{L}$ dNTP mix (10 mM), 0.8 units GO Taq DNA polymerase and $35.25 \mu \mathrm{L}$ water. Cycling conditions consisted of 1 cycle for $2 \mathrm{~min}$ at $94^{\circ} \mathrm{C}, 35$ cycles of $30 \mathrm{sec}$ at $94^{\circ} \mathrm{C}, 30 \mathrm{sec}$ at $55^{\circ} \mathrm{C}$ and $3 \mathrm{~min}$ at $72^{\circ} \mathrm{C}$, and a final extension step for $10 \mathrm{~min}$ at $72^{\circ} \mathrm{C}$. PCR products were purified 
and sequenced. The obtained sequences were used as query to blast against the V. dahliae genome (http://fungi.ensembl.org/Verticillium_dahliaejr2/Info/Index) (Faino et al. 2015).

\section{Targeted mutagenesis and complementation}

To generate a VdNRS/ER deletion construct, sequences flanking the VdNRS/ER coding sequence were amplified from genomic DNA of $V$. dahliae strain JR2 using the primers KO-6010-LF with KO-6010-LR to amplify the left border, and KO-6010RF with KO-6010-RR to amplify the right border, respectively (Supplemental Table 1). The resulting amplicons were cloned into pRF-HU2 as described previously (Frandsen et al. 2008). A. tumefaciens-mediated transformation of $V$. dahliae was performed as described previously (Santhanam 2012), and transformants were selected on PDA supplemented with $200 \mu \mathrm{g} / \mathrm{mL}$ of cefotaxime (Duchefa, Haarlem, The Netherlands), $50 \mu \mathrm{g} / \mathrm{mL}$ of hygromycin (Duchefa, Haarlem, The Netherlands). Homologous recombination was verified by PCR.

To generate a VdNRS/ER complementation construct, a 2404 bp EcoRI/Pacl fragment containing the VDAG_06010 (VDAG_JR2_Chr7g02960) coding sequence with 1000 bp upstream and 450 bp downstream sequence was amplified from $V$. dahliae strain JR2 genomic DNA, and cloned into binary vector pBT081 (Houterman et al. 2008). Complementation transformants were selected on PDA supplemented with $200 \mu \mathrm{g} /$ $\mathrm{mL}$ of cefotaxime and $100 \mu \mathrm{g} / \mathrm{mL}$ phleomycin (InvivoGen, San Diego, USA).

\section{Growth, conidiogenesis and stress assays}

Radial growth was monitored by placing a $2 \mu \mathrm{L}$ droplet of a conidial suspension of $10^{6}$ conidiospores $/ \mathrm{mL}$ in the centre of PDA or Czapek-dox medium, incubated at $22^{\circ} \mathrm{C}$ and the colony diameter was measured after 10 days. For quantification of conidiospore production, $5 \mathrm{~mL}$ of water was added to the culture and a conidial suspension was prepared by gently rubbing the mycelium. A ten-fold dilution of the conidial suspension was counted using a haemocytometer. Stress sensitivity assays were performed by placing a $2 \mu \mathrm{L}$ droplet with $10^{6}$ conidiospores $/ \mathrm{mL}$ of wild type $V$. dahliae, two VdNRS/ER deletion strains and an ectopic transformant in the centre of a Czapek-dox plate supplemented with congo red $(250 \mathrm{mM}, 500 \mathrm{mM}, 750 \mathrm{mM}$ and $1 \mathrm{M}), \mathrm{NaCl}(250 \mathrm{mM}, 500 \mathrm{mM}, 750 \mathrm{mM}$ and $1 \mathrm{M})$, mannitol $(250 \mathrm{mM}, 500 \mathrm{mM}$, $750 \mathrm{mM}$ and $1 \mathrm{M}$ ), or sorbitol $(300 \mathrm{mM}, 600 \mathrm{mM}, 900 \mathrm{mM}, 1.2 \mathrm{M})$, and incubated at $22^{\circ} \mathrm{C}$. Plates were photographed at $7 \mathrm{dpi}$ and the colony diameter was measured using ImageJ software. 


\section{Root colonization assay}

The root colonization assay was performed as previously described (Di Pietro et al. 2001). Briefly, the roots of 10-day-old tomato seedlings were rinsed in water and placed in Erlenmeyer flasks containing a suspension of $10^{6}$ conidia $/ \mathrm{mL}$ in onefifth PDB and incubated at $22^{\circ} \mathrm{C}$ and 100 r.p.m. Fungal colonization of the root surface was observed macroscopically from 3 up to 5 days post inoculation. The experiments were performed three times with similar results.

\section{Expression of Recombinant VdNRS/ER}

The following expression constructs were expressed in E. coli strain BI21(DE3): pET6hSUMO-VDAG_06010, contains a 6-histidine-SUMO tag fused to the N-terminal of $V$. dahliae VdNRS/ER (VDAG_06010); pET-6hSUMO was used as a empty vector control (pET-SUMO); pET28-BfDH4.1 (Martinez et al. 2012) was used to express and purify the 6 His tagged Botrytis fuckeliana UDP-Glc 4,6-dehydratase (BfDH). The E. coli strains carrying the different constructs were cultured for $16 \mathrm{~h}$ at $37^{\circ} \mathrm{C}$ in LB medium supplemented with $50 \mu \mathrm{g} / \mathrm{mL}$ kanamycin and $35 \mu \mathrm{g} / \mathrm{mL}$ chloramphenicol. The following day, $5 \mathrm{~mL}$ cultures were transferred into $245 \mathrm{~mL}$ of fresh LB liquid medium supplemented with the same antibiotics and cell growth was resumed at $37^{\circ} \mathrm{C}$ at $220 \mathrm{rpm}$ until the cell density reached an $\mathrm{OD}_{600}$ of $\sim 0.6$. Gene expression was then induced by the addition of isopropyl $\beta$-D-thiogalactoside $(0.5 \mathrm{~mm})$, and cultures were grown for additional $20 \mathrm{~h}$ at $18^{\circ} \mathrm{C}$ at $220 \mathrm{rpm}$. Cells were collected by centrifugation $\left(6,000 \times \mathrm{g}\right.$ for $10 \mathrm{~min}$ at $\left.4^{\circ} \mathrm{C}\right)$ and suspended in $10 \mathrm{~mL}$ lysis buffer [50 mm Tris- $\mathrm{HCl}, \mathrm{pH}$ 7.5, 10\% glycerol, 1 mm EDTA, 2 mM DTT, $0.5 \mathrm{mM}$ PMSF, with or without $50 \mathrm{~mm} \mathrm{NaCl}$. Cell were lysed in an ice bath with a S-4000 sonicator (Misonix Inc, Farmingdale, NY, USA) equipped with 1/8-inch microtip probe using 12 sonication cycles each (10-s pulse; 20 -s off). The lysed cells were centrifuged $\left(6,000 \times g\right.$ for $10 \mathrm{~min}$ at $\left.4^{\circ} \mathrm{C}\right)$; the supernatant was supplemented with $1 \mathrm{~mm}$ DTT and centrifuged again $\left(20,000 \times \mathrm{g}\right.$ for $30 \mathrm{~min}$ at $\left.4^{\circ} \mathrm{C}\right)$. The resulting supernatant was kept at $-20^{\circ} \mathrm{C}$. Protein purification was carried out as previously described (Martinez et al. 2012).

\section{Enzyme Assays}

The dual activity of recombinant enzymes BfDH and VdNRS/ER was analyzed by LC-MS, in a total volume of $50 \mu \mathrm{l}$, containing $50 \mathrm{mM}$ Tris- $\mathrm{HCl} \mathrm{pH} 6.5,0.25 \mathrm{mM}$ UDP-Glc, $0.5 \mathrm{mM} \mathrm{NAD}+0.5 \mathrm{mM} \mathrm{NADPH}, 5 \mu \mathrm{l}$ of purified B. fuckeliana BfDH with or without the addition of $2 \mu \mathrm{l}$ of $V d N R S / E R$. The reaction was incubated at $37^{\circ} \mathrm{C}$ for $1 \mathrm{~h}$ and terminated by heating for $5 \mathrm{~min}$ at $95^{\circ} \mathrm{C}$. After the addition of chloroform and centrifugation $(10,000 \times \mathrm{g} 4 \mathrm{~min})$ an aliquot (20 $\mu \mathrm{l}$ ) of the upper layer phase was 
removed and mixed with $38 \mu \mathrm{l}$ acetonitrile and $2 \mu \mathrm{l} 0.5 \mathrm{M}$ ammonium-acetate $\mathrm{pH}$ 4.35. A portion ( $25 \mu \mathrm{l}$ ) of this mixture was analyzed using HILIC chromatography and mass spectrometry analyses. This was done using a LC-MS/MS IT-TOF MS system (Shimadzu Scientific Instruments, Columbia, MD, USA) operating in the negative ion mode with a Nexera UFPLC LC-30AD pump, autosampler (Sil30), and column heater (set to $37^{\circ} \mathrm{C}$ ). Nucleotide sugars were separated on an Accucore 150-amid HILIC column ( $150 \times 4.6 \mathrm{~mm}, 2.6 \mu \mathrm{m}$ particle size, ThermoScientific) using $40 \mathrm{mM}$ ammonium acetate, $\mathrm{pH} 4.3$ (solvent A), and acetonitrile (solvent $\mathrm{B}$ ) with the gradient conditions as in (Li et al. 2015).

\section{Isolation of polysaccharide and sugar analyses by GC-MS}

Conidiospores were harvested from 7-day-old $V$. dahliae cultures grown on potato dextrose agar (PDA) (Thermo Fisher Scientific Inc, Breda, The Netherlands). Germinating spores were prepared by inoculating $30 \mu \mathrm{l}$ of conidiospores into $12.5 \mathrm{~mL}$ of Gamborg's Glc (2\%) in a medium sized plate $(9 \mathrm{~cm})$. The plates were incubated for 66 hours at room temperature under regular light to allow the spores to germinate. Polyssaccharides were either directly extracted from conidospore samples or from germinating conidiospores $(<100 \mu \mathrm{g})$ starting by incubation for $3 \mathrm{~h}$ at $80^{\circ} \mathrm{C}$ while stirring with $5 \mathrm{~mL} 1 \mathrm{M} \mathrm{NaOH}(5 \mathrm{~mL})$. After centrifugation $(10,000 \times \mathrm{g}$, $4^{\circ} \mathrm{C}, 10 \mathrm{~min}$ ) the alkaline extract was mixed with an equal volume of $96 \%$ ethanol. Following centrifugation $\left(10,000 \times \mathrm{g}, 4^{\circ} \mathrm{C}, 15 \mathrm{~min}\right)$, the pellet was resuspended in $10 \mathrm{~mL}$ water, and dialyzed (3,000 molecular weight cutoff) against two 10-liter changes of deionized water. The dialyzed solution was centrifuged $\left(10,000 \times 9,4^{\circ} \mathrm{C}\right.$, $15 \mathrm{~min}$ ) and the water-soluble supernatant (aWS) was collected and freeze-dried. The glycosyl residue compositions of these extracts were then determined.

An aliquot of each extract $(1 \mathrm{mg})$ was supplemented with $10 \mu \mathrm{g}$ inositol and hydrolyzed with $1 \mathrm{~mL}$ of $2 \mathrm{M}$ trifluoroacetic acid at $120^{\circ} \mathrm{C}$. The released monosaccharides were reduced to their alditols (York et al. 1986), and acetylated. The resulting alditol acetate derivatives were analyzed by GC-MS system consisting of GC (Agilent 7890a) (Agilent Technologies, Santa Clara, CA, USA) equipped with an El-MS detector (5975c). A $1 \mu \mathrm{l}$ sample or std was injected via Agilent 7693 autosampler injector port (Agilent Technologies, Santa Clara, CA, USA) (set at $250^{\circ} \mathrm{C} ; 3 \mathrm{~mL} / \mathrm{min}$ helium) in a split (1:50) mode into a non-polar Equity-1 capillary column (30 m x 0.25 $\mathrm{mm}$ i.d.; $0.25 \mu \mathrm{m}$ film thickness). The chromatography gas carrier was helium ( $1 \mathrm{~mL} /$ $\mathrm{min}$ ) and the column oven temperature program used to separate sugar derivatives was as follows: initial injection temperature was $60^{\circ} \mathrm{C}$ for $1 \mathrm{~min}$, followed by an increase of $27.5^{\circ} \mathrm{C} \mathrm{min}{ }^{-1}$ to $170^{\circ} \mathrm{C}$, then at $4^{\circ} \mathrm{C} \mathrm{min}{ }^{-1}$ to $235^{\circ} \mathrm{C}$, and a hold at $235^{\circ} \mathrm{C}$ for $2 \mathrm{~min}$. The column was then kept at $260^{\circ} \mathrm{C}$ for $12 \mathrm{~min}$, cooled to $60^{\circ} \mathrm{C}$ and kept 
at $60^{\circ} \mathrm{C}$ for 1 min prior to the next sample injection. The temperature of the transfer line between the column ends to the MS was $250^{\circ} \mathrm{C}$. Detection was achieved using El-MS operating with an electron impact ionization of energy $70 \mathrm{~V}$ with the temperature of $\mathrm{MS}$ ion source set at $230^{\circ} \mathrm{C}$ and that of the quad at $150^{\circ} \mathrm{C}$. MS data was collected in full scan monitoring mode at $\mathrm{m} / \mathrm{z}$ range $50-550$ from $5-50 \mathrm{~min}$. The spectra were analyzed using Software MSD ChemStation D.02.00.275 (Agilent Technologies, Santa Clara, CA, USA). Alditol-acetate derivatives of monosaccharide standards (50 $\mathrm{\mu g}$ each of rhamnose, fucose, xylose, mannose, glucose, and $30 \mathrm{\mu g}$ each ribose, arabinose, galactose) supplemented with $10 \mu \mathrm{g}$ inositol (internal std.) were prepared under the same conditions as the samples. For data analysis, sugar residue peaks were identified based on retention times of standard monosaccharides and their characteristic mass spectrum.

\section{Acknowledgements}

The authors thank Hugo de Vries and Emilie Fradin for technical assistance, and Bert Essenstam and Henk Smid for excellent plant care. B.P.H.J.T. is supported by a Vici grant of the Research Council for Earth and Life sciences (ALW) of the Netherlands Organization for Scientific Research (NWO). This research was funded in part by BARD grant \# IS 4501-12 to M.B.P. 


\section{References}

Bolek, Y., El-Zik, K. M., Pepper, A. E., Bell, A. A., Magill, C. W., Thaxton, P. M., and Reddy, O. U. K. 2005. Mapping of Verticillium wilt resistance genes in cotton. Plant Sci 168:1581-1590.

Chiang, S. L., and Mekalanos, J. J. 1999. rfb mutations in Vibrio cholerae do not affect surface production of toxin-coregulated pili but still inhibit intestinal colonization. Infect Immun 67:976-980.

de Jonge, R., Bolton, M. D., Kombrink, A., van den Berg, G. C., Yadeta, K. A., and Thomma, B. P. H. J. 2013. Extensive chromosomal reshuffling drives evolution of virulence in an asexual pathogen. Genome Res 23:1271-1282.

de Jonge, R., van Esse, H. P., Maruthachalam, K., Bolton, M. D., Santhanam, P., Saber, M. K., Zhang, Z., Usami, T., Lievens, B., Subbarao, K. V., and Thomma, B. P. H. J. 2012. Tomato immune receptor Ve1 recognizes effector of multiple fungal pathogens uncovered by genome and RNA sequencing. Proc Natl Acad Sci U S A 109:5110-5115.

Di Pietro, A., Garcia-MacEira, F. I., Meglecz, E., and Roncero, M. I. 2001. A MAP kinase of the vascular wilt fungus Fusarium oxysporum is essential for root penetration and pathogenesis. Mol Microbiol 39:1140-1152.

Faino, L., de Jonge, R., and Thomma, B. P. H. J. 2012. The transcriptome of Verticillium dahliae-infected Nicotiana benthamiana determined by deep RNA sequencing. Plant Signal Behav 7:1065-1069.

Faino, L., Seidl, M. F., Datema, E., van den Berg, G. C., Janssen, A., Wittenberg, A. H., and Thomma, B. P. H. J. 2015. Single-Molecule Real-Time Sequencing Combined with Optical Mapping Yields Completely Finished Fungal Genome. MBio 6.

Fradin, E. F., and Thomma, B. P. H. J. 2006. Physiology and molecular aspects of Verticillium wilt diseases caused by V. dahliae and V. albo-atrum. Mol Plant Pathol 7:71-86.

Fradin, E. F., Zhang, Z., Juarez Ayala, J. C., Castroverde, C. D., Nazar, R. N., Robb, J., Liu, C. M., and Thomma, B. P. H. J. 2009. Genetic dissection of Verticillium wilt resistance mediated by tomato Ve1. Plant Physiol 150:320-332.

Frandsen, R. J., Andersson, J. A., Kristensen, M. B., and Giese, H. 2008. Efficient four fragment cloning for the construction of vectors for targeted gene replacement in filamentous fungi. BMC Mol Biol 9:70.

Gao, M., D'Haeze, W., De Rycke, R., Wolucka, B., and Holsters, M. 2001. Knockout of an azorhizobial dTDP-L-rhamnose synthase affects lipopolysaccharide and extracellular polysaccharide production and disables symbiosis with Sesbania rostrata. Mol. Plant Microbe Interact. 14:857-866.

Giesbert, S., Schumacher, J., Kupas, V., Espino, J., Segmuller, N., Haeuser-Hahn, I., Schreier, P. H., and Tudzynski, P. 2012. Identification of pathogenesis-associated genes by T-DNA-mediated insertional mutagenesis in Botrytis cinerea: a type 2A phosphoprotein phosphatase and an SPT3 transcription factor have significant impact on virulence. Mol. Plant Microbe Interact. 25:481-495.

Giraud, M. F., Leonard, G. A., Field, R. A., Berlind, C., and Naismith, J. H. 2000. RmlC, the third enzyme of dTDP-L-rhamnose pathway, is a new class of epimerase. Nat Struct Mol Biol 7:398-402.

Hayes, R. J., McHale, L. K., Vallad, G. E., Truco, M. J., Michelmore, R. W., Klosterman, S. J., Maruthachalam, K., and Subbarao, K. V. 2011. The inheritance of resistance to Verticillium wilt caused by race 1 isolates of Verticillium dahliae in the lettuce cultivar La Brillante. Theor Appl Genet 123:509-517.

Houterman, P. M., Cornelissen, B. J., and Rep, M. 2008. Suppression of plant resistance gene-based immunity by a fungal effector. PLoS Pathog 4:e1000061.

Hüser, A., Takahara, H., Schmalenbach, W., and O'Connell, R. 2009. Discovery of pathogenicity genes in the crucifer anthracnose fungus Colletotrichum higginsianum, using random insertional mutagenesis. Mol. Plant Microbe Interact. 22:143-156.

Jeon, J., Park, S. Y., Chi, M. H., Choi, J., Park, J., Rho, H. S., Kim, S., Goh, J., Yoo, S., Choi, J., Park, J. Y., Yi, M., Yang, S., Kwon, M. J., Han, S. S., Kim, B. R., Khang, C. H., Park, B., Lim, S. E., Jung, K., Kong, S., Karunakaran, M., Oh, H. S., Kim, H., Kim, S., Park, J., Kang, S., Choi, W. B., Kang, S., and Lee, Y. H. 2007. Genome-wide functional analysis of pathogenicity genes in the rice blast fungus. Nat Genet 39:561-565. 
Jofré, E., Lagares, A., and Mori, G. 2004. Disruption of dTDP-rhamnose biosynthesis modifies lipopolysaccharide core, exopolysaccharide production, and root colonization in Azospirillum brasilense. FEMS Microbiol Lett 231:267-275.

Klimes, A., Dobinson, K. F., Klosterman, S. J., and Thomma, B. P. H. J. 2015. Genomics spurs rapid advances in our understanding of the basic biology of vascular wilt pathogens in the genus Verticillium. Annu Rev Phytopathol 53:181-198.

Klosterman, S. J., Atallah, Z. K., Vallad, G. E., and Subbarao, K. V. 2009. Diversity, pathogenicity, and management of Verticillium species. Annu Rev Phytopathol 47:39-62.

Klosterman, S. J., Subbarao, K. V., Kang, S., Veronese, P., Gold, S. E., Thomma, B. P. H. J., Chen, Z., Henrissat, B., Lee, Y. H., Park, J., Garcia-Pedrajas, M. D., Barbara, D. J., Anchieta, A., de Jonge, R., Santhanam, P., Maruthachalam, K., Atallah, Z., Amyotte, S. G., Paz, Z., Inderbitzin, P., Hayes, R. J., Heiman, D. I., Young, S., Zeng, Q., Engels, R., Galagan, J., Cuomo, C. A., Dobinson, K. F., and Ma, L. J. 2011. Comparative genomics yields insights into niche adaptation of plant vascular wilt pathogens. PLoS Pathog 7:e1002137.

Korn, M., Schmidpeter, J., Dahl, M., Muller, S., Voll, L. M., and Koch, C. 2015. A genetic screen for pathogenicity genes in the hemibiotrophic fungus Colletotrichum higginsianum identifies the plasma membrane proton pump pma2 required for host penetration. PLoS One 10:e0125960.

Li, Z., Hwang, S., Ericson, J., Bowler, K., and Bar-Peled, M. 2015. Pen and Pal are nucleotide-sugar dehydratases that convert UDP-GIcNac to UDP-6-deoxy-D-GlcNac-5, 6-ene and then to UDP-4keto-6-deoxy-L-AltNac for CMP-Pseudaminic acid synthesis in Bacillus thuringiensis. J Biol Chem 290:691-704.

Martinez, V., Ingwers, M., Smith, J., Glushka, J., Yang, T., and Bar-Peled, M. 2012. Biosynthesis of UDP-4keto-6-deoxyglucose and UDP-rhamnose in pathogenic fungi Magnaporthe grisea and Botryotinia fuckeliana. J Biol Chem 287:879-892.

Maruthachalam, K., Klosterman, S. J., Kang, S., Hayes, R. J., and Subbarao, K. V. 2011. Identification of pathogenicity-related genes in the vascular wilt fungus Verticillium dahliae by Agrobacterium tumefaciens-mediated T-DNA insertional mutagenesis. Mol Biotechnol 49:209-221.

Meng, Y., Patel, G., Heist, M., Betts, M. F., Tucker, S. L., Galadima, N., Donofrio, N. M., Brown, D., Mitchell, T. K., Li, L., Xu, J. R., Orbach, M., Thon, M., Dean, R. A., and Farman, M. L. 2007. A systematic analysis of T-DNA insertion events in Magnaporthe oryzae. Fungal Genet Biol 44:10501064.

Michielse, C. B., van Wijk, R., Reijnen, L., Cornelissen, B. J., and Rep, M. 2009. Insight into the molecular requirements for pathogenicity of Fusarium oxysporum f. sp. lycopersici through large-scale insertional mutagenesis. Genome Biol 10:R4.

Mullins, E., Chen, X., Romaine, P., Raina, R., Geiser, D., and Kang, S. 2001. Agrobacterium-mediated transformation of Fusarium oxysporum: an efficient tool for insertional mutagenesis and gene transfer. Phytopathology 91:173-180.

Munch, S., Ludwig, N., Floss, D. S., Sugui, J. A., Koszucka, A. M., Voll, L. M., Sonnewald, U., and Deising, H. B. 2011. Identification of virulence genes in the corn pathogen Colletotrichum graminicola by Agrobacterium tumefaciens-mediated transformation. Mol Plant Pathol 12:43-55.

Nicholson, R. L., and Kunoh, H. 1995. Early interactions, adhesion, and establishment of the infection court by Erysiphe graminis. Canadian Journal of Botany 73:609-615.

Oka, T., Nemoto, T., and Jigami, Y. 2007. Functional analysis of Arabidopsis thaliana RHM2/MUM4, a multidomain protein involved in UDP-D-glucose to UDP-L-rhamnose conversion. J Biol Chem 282:5389-5403.

Pegg, G. F., and Brady, B. L. 2002. Verticillium wilts. CABI, Wallingford.

Ramadoss, C. S., Uhlig, J., Carlson, D. M., Butler, L. G., and Nicholson, R. L. 1985. Composition of the mucilaginous spore matrix of Colletotrichum graminicola, a pathogen of corn, sorghum, and other grasses. J Agric Food Chem 33:728-732. 
Ramos, B., Gonzalez-Melendi, P., Sanchez-Vallet, A., Sanchez-Rodriguez, C., Lopez, G., and Molina, A. 2013. Functional genomics tools to decipher the pathogenicity mechanisms of the necrotrophic fungus Plectosphaerella cucumerina in Arabidopsis thaliana. Mol Plant Pathol 14:44-57.

Rowe, R. C., Davis, J. R., Powelson, M. L., and Rouse, D. I. 1987. Potato early dying: causal agents and management strategies. Plant Dis 71:482-489.

Santhanam, P. 2012. Random insertional mutagenesis in fungal genomes to identify virulence factors. Pages 509-517 in: Plant fungal pathogens. Methods in molecular biology, vol. 835. T. B. Bolton MD, ed. Humana press, Totowa.

Santhanam, P., and Thomma, B. P. H. J. 2013. Verticillium dahliae Sge1 differentially regulates expression of candidate effector genes. Mol. Plant Microbe Interact. 26:249-256.

Santhanam, P., van Esse, H. P., Albert, I., Faino, L., Nurnberger, T., and Thomma, B. P. H. J. 2013. Evidence for functional diversification within a fungal NEP1-like protein family. Mol. Plant Microbe Interact. 26:278-286.

Schaible, L., Cannon, O. S., and Waddoups, V. 1951. Inheritance of resistance to Verticillium wilt in a tomato cross. Phytopathology 41:986-990.

Schumacher, J., Simon, A., Cohrs, K. C., Viaud, M., and Tudzynski, P. 2014. The transcription factor BcLTF1 regulates virulence and light responses in the necrotrophic plant pathogen Botrytis cinerea. PLoS Genet 10:e1004040.

Simko, I., Haynes, K. G., Ewing, E. E., Costanzo, S., Christ, B. J., and Jones, R. W. 2004. Mapping genes for resistance to Verticillium albo-atrum in tetraploid and diploid potato populations using haplotype association tests and genetic linkage analysis. Mol Genet Genomics 271:522-531.

Singh, S., Braus-Stromeyer, S. A., Timpner, C., Tran, V. T., Lohaus, G., Reusche, M., Knufer, J., Teichmann, T., von Tiedemann, A., and Braus, G. H. 2010. Silencing of Vlaro2 for chorismate synthase revealed that the phytopathogen Verticillium longisporum induces the cross-pathway control in the xylem. Appl Microbiol Biotechnol 85:1961-1976.

Sugui, J. A., Kunoh, H., and Nicholson, R. L. 1999. Detection of protein and carbohydrate in the extracellular matrix released by Cochliobolus heterostrophus during germination. Mycoscience 40:11-19.

Thomma, B. P. H. J., Nurnberger, T., and Joosten, M. H. A. J. 2011. Of PAMPs and effectors: The blurred PTI-ETI dichotomy. Plant Cell 23:4-15.

Timpner, C., Braus-Stromeyer, S. A., Tran, V. T., and Braus, G. H. 2013. The Cpc1 regulator of the crosspathway control of amino acid biosynthesis is required for pathogenicity of the vascular pathogen Verticillium longisporum. Mol. Plant Microbe Interact. 26:1312-1324.

Tzima, A. K., Paplomatas, E. J., Rauyaree, P., Ospina-Giraldo, M. D., and Kang, S. 2011. VdSNF1, the sucrose nonfermenting protein kinase gene of Verticillium dahliae, is required for virulence and expression of genes involved in cell-wall degradation. Mol. Plant Microbe Interact. 24:129-142.

Watt, G., Leoff, C., Harper, A. D., and Bar-Peled, M. 2004. A bifunctional 3, 5-epimerase/4-keto reductase for nucleotide-rhamnose synthesis in Arabidopsis. Plant Physiol 134:1337-1346.

Wilhelm, S. 1955. Longevity of the Verticillium wilt fungus in the laboratory and field. Phytopathology 45:180-181.

Winnenburg, R., Baldwin, T. K., Urban, M., Rawlings, C., Köhler, J., and Hammond-Kosack, K. E. 2006. PHI-base: a new database for pathogen host interactions. Nucleic Acids Res 34:D459-D464.

Winnenburg, R., Urban, M., Beacham, A., Baldwin, T. K., Holland, S., Lindeberg, M., Hansen, H., Rawlings, C., Hammond-Kosack, K. E., and Kohler, J. 2008. PHI-base update: additions to the pathogen host interaction database. Nucleic Acids Res 36:D572-576.

$\mathrm{Xu}$, L., and Chen, W. 2013. Random T-DNA mutagenesis identifies a Cu/Zn superoxide dismutase gene as a virulence factor of Sclerotinia sclerotiorum. Mol. Plant Microbe Interact. 26:431-441.

York, W. S., Darvill, A. G., McNeil, M., Stevenson, T. T., and Albersheim, P. 1986. Isolation and characterization of plant cell walls and cell wall components. Meth Enzymol 118:3-40. 


\section{Supplemental data}

SUPPLEMENTAL TABLE 1 | Primers used in this study

\begin{tabular}{|c|c|c|}
\hline Primer & Sequence $\left(5^{\prime}-3^{\prime}\right)$ & Remarks \\
\hline MLBF & GGATTTTGGTTTTAGGAATTAGA & Mspl left border, forward \\
\hline MLBR & AATTCGGCGTTAATTCAGTACA & Mspl left border, reverse \\
\hline MLSeq & TCAGTACATTAAAAACGTCCGCAA & Mspl left border, sequencing \\
\hline MRBF & CAACTGTTGGGAAGGGCGATC & Mspl right border, forward \\
\hline MRBR & CAGCCTGAATGGCGAATGCTA & Mspl right border, reverse \\
\hline MRSeq & GAATGCTAGAGCAGCTTGAGCT & Mspl right border, sequencing \\
\hline NLBF & AGTGTATTGACCGATTCCTTGC & Ncol left border, forward \\
\hline NLBR & AGGGTTCCTATAGGGTTTCGCTCATG & Ncol left border, reverse \\
\hline NLSeq & GAATTAATTCGGCGTTAATTCAGT & Ncol left border, sequencing \\
\hline NRBF & CGTTATGTTTATCGGCACTTTG & Ncol right border, forward \\
\hline NRBR & GGCACTGGCCGTCGTTTTACAAC & Ncol right border, reverse \\
\hline NRSeq & СССTTCCCAACAGTTGCGCA & Ncol right border, sequencing \\
\hline KO-6010-LF & GGTCTTAAUACTGCGCTGATGACCTCAC & Left border, forward \\
\hline KO-6010-LR & GGCATTAAUCAGTGGATGCTGTTGTCGAT & Left border, reverse \\
\hline KO-6010-RF & GGACTTAAUAATGCGAAATGCGTAAGGAG & Right border, forward \\
\hline KO-6010-RR & GGGTTTAAUGCGGTAGGCCTTCTTGATCT & Right border, reverse \\
\hline 6010-F & GCGGCCGCATGTCAGTTACGAACGGTGC & Full length, forward \\
\hline $6010-R$ & CCATGGTCAATTAACACCAGCAGCCTT & Full length, reverse \\
\hline 6010 -comp_F & GAATTCTGCGCTGATGACCTCACC & Complementation, forward \\
\hline 6010 -comp_R & TTAATTAACCGCCTTGGATCACCAC & Complementation, reverse \\
\hline qPCR-6010-F & ACACGATCCTCCACG ATCTC & Real-time PCR, forward \\
\hline qPCR-6010-R & GCGAGAAGTTCTTCCAGGTG & Real-time PCR, reverse \\
\hline VdELF1-a-F & CCATTGATATCGCACTGTGG & Real-time PCR, forward \\
\hline VdELF1-a-F & TGGAGATACCAGCCTCGAAC & Real-time PCR, reverse \\
\hline qPCR-Hyg-F & ATAGGTCAGGCTCTCGCTGA & Real-time PCR, forward \\
\hline qPCR-Hyg-R & GATGTAGGAGGGCGTGGATA & Real-time PCR, reverse \\
\hline 6010-BamH1-F & GGATCCTCAGTTACGAACGGTGCCA & pET-SUMO cloning, forward \\
\hline 6010-Hind3-R & AAGCTTCTATTATCAATTAACACCAGCAGCCTT & pET-SUMO cloning, reverse \\
\hline
\end{tabular}


A

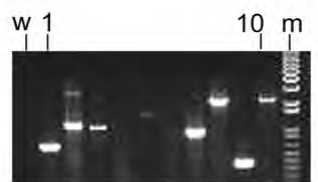

B

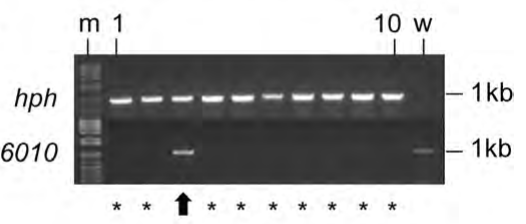

C

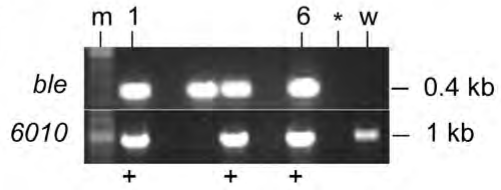

SUPPLEMENTAL FIGURE 1 | Verification of deletion and complementation strains by PCR. (A) Amplification of the T-DNA flanking sequences from wild type $V$. dahliae $(w), 10$ independent random transformants (lanes 1 to 10), and the marker (m) is indicated. (B) Amplification of the hygromycin resistance gene (hph) and VdNRS/ER from 10 independent transformants (lanes 1 to 10 ) and wild type $V$. dahliae $(w)$. The marker $(m)$ is indicated. True deletion strains are marked with asterisks, while the ectopic transformant is marked with an arrow. (C) Amplification of the zeocin resistance gene (b/e) and $V d N R S / E R$ from 5 independent complementation strains (lanes 1 to 5), a VdNRS/ER deletion strain (*) and wild type $V$. dahliae $(\mathrm{w})$. The marker $(\mathrm{m})$, and true complementation strains $(+)$ are indicated.
A

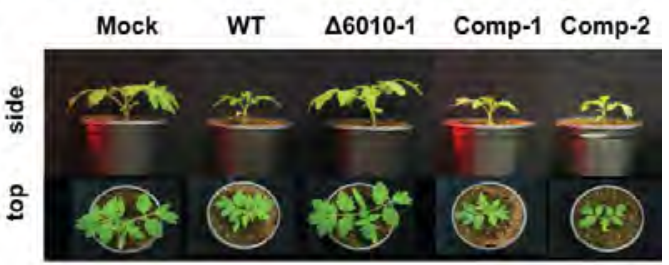

B

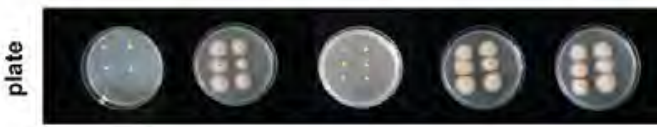

C

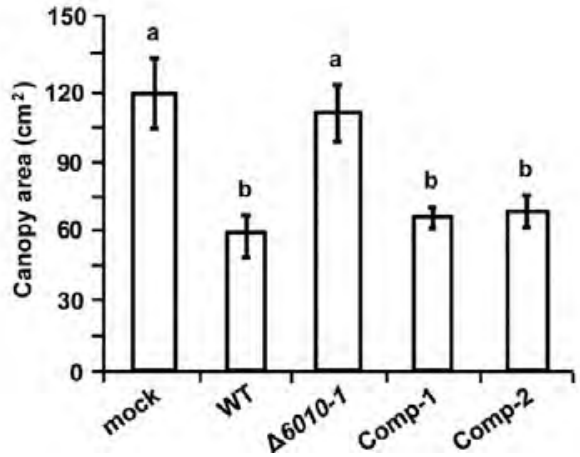

SUPPLEMENTAL FIGURE 2 | Complementation of VdNRS/ER deletion strain restores pathogenicity on tomato. (A) Side and top view of tomato cultivar MoneyMaker plants inoculated with wild type $V$. dahliae (WT), a VdNRS/ER deletion strain $(\triangle 6010-1)$ and two complementation strains (comp-1 and comp-2), or mock-inoculated at 14 days post inoculation. (B) Fungal outgrowth at 7 days after plating of stem sections harvested at 14 days post inoculation. (C) Average canopy area of 6 plants at 14 days after inoculation as described for panel A. Different letter labels indicate significant differences $(P<0.05)$. 
General discussion:

From effector discovery to breeding for durable pathogen resistance in crops

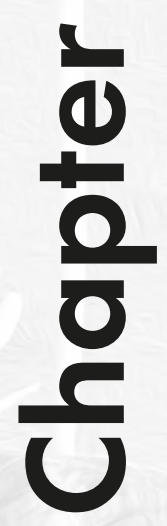


One of the major challenges in agriculture is to prevent yield loss caused by plant pathogens. Arguably, the most sustainable way to control disease is the deployment of genetic resistance. Pathogen resistance in plants is often conferred by immune receptors that, directly or indirectly, recognize pathogens or their activity. Effectors are pathogen-secreted molecules that support host colonization and therefore pose a strong selection pressure on plant hosts to recognize them. Effector identification and characterization has shed light on our understanding of plant-pathogen interactions, and on the various molecular mechanisms of pathogens and hosts that are involved in shaping those interactions. Here, I discuss the current understanding of pathogen effectors and how they can be exploited in breeding programs to establish durable pathogen resistance in crops. 


\section{Introduction}

Crop production is continuously threatened by plant pathogens, and genetic resistance is the most important source to prevent disease development. Already in the $40^{\prime}$ s of the $20^{\text {th }}$ century, Harold Flor proposed the "gene-for-gene" theory, stating that for any given plant that carries a resistance $(R)$ gene, a corresponding avirulence (Avr) gene is present in the pathogen to which it confers resistance (Flor 1942). This led to the assumption that $\mathrm{R}$ proteins directly interact with the corresponding Avr proteins (Keen 1990). However, most studies on R-Avr pairs failed to demonstrate such direct interaction, which led to the formulation of "the guard hypothesis" (Van der Biezen and Jones 1998). This hypothesis states that plant $R$ proteins monitor, or "guard", the status of a plant protein that may be targeted by pathogen effectors. Once this indeed happens and the "guardee" is modulated by a pathogen effector, the guard becomes activated and triggers an immune response. This hypothesis was formulated because meanwhile it had become evident that Avr proteins often have functions to contribute to the virulence of the pathogen, for instance by targeting host proteins that are involved in defence responses, and were therefore referred to as effectors (Boller and Felix 2009). Currently, pathogen effectors are best described as secreted molecules that support host colonization (Rovenich et al. 2014). Besides pathogen effectors, plant immune receptors may also recognize conserved microbial signatures such as fungal chitin and bacterial flagellin, also known as microbe-associated molecular patterns (MAMPs). Whereas recognition of effectors is referred to as effector-triggered immunity (ETI), recognition of MAMPs is referred to as MAMP-triggered immunity (MTI) in the so-called "zigzag model" (Chisholm et al. 2006; Jones and Dangl 2006). It is generally accepted that successful plant pathogens use effectors to overcome MTI. In turn, to overcome ETI upon recognition of an effector by a host receptor, the pathogen needs to mutate or purge this effector, or evolve a novel effector to suppress the immune response and successfully colonize the plant.

\section{Effectors and MAMPs cannot be strictly separated}

The discovery and characterization of various effectors and MAMPs from plant pathogens, including fungi, oomycetes and bacteria, have resulted in molecules that cannot be strictly separated as was proposed in "the zigzag model" (Thomma et al. 2011). In this model, effectors are described as species-, race- or even isolatespecific and contribute to pathogen virulence, whereas MAMPs are described as molecular patterns that are conserved in a broad range of species and contribute to microbial fitness. Arguably, these two groups represent the ends of a spectrum of molecules that should be seen as a continuum. Indeed, these definitions do not apply to pathogen molecules such as the NEP1-like proteins (NLPs) that are 
found in a multitude of species belonging even to multiple kingdoms, and act as effectors that stimulate virulence on the one hand, and as MAMP on the other hand (Oome et al. 2014; Pemberton and Salmond 2004). Similarly, lysin-motif containing (LysM) effectors are conserved and widespread in the fungal kingdom (de Jonge and Thomma 2009; Kombrink and Thomma 2013). Also Verticillium dahliae effector Ave1 has homologs in other plant pathogens, including the fungi Fusarium oxysporum, Colletotrichum higginsianum, Cercospora beticola and the bacterium Xanthomonas axonopodis (de Jonge et al. 2012). In these organisms, the Ave1 homologs act as virulence factor as well (Ficarra et al. 2017; Gottig et al. 2008), with the exception of C. higginsianum (Chapter 3, this thesis). In addition, multiple Ave1 homologs were identified in the fungi Venturia inaequalis and V. pirina that are pathogens of apple and pear, respectively (Chapter 5, this thesis)(Cooke et al. 2014; Deng et al. 2017). However, whether these homologs act as virulence factors too remains to be demonstrated. Tomato immune receptor Ve1 differentially recognizes Ave1 effector homologs from various plant pathogens (Chapter, 4 this thesis)(de Jonge et al. 2012; Song et al. 2017). Finally, the effector proteins Pep1 and Pee1 of the maize smut fungus Ustilago maydis, which are essential effectors at the biotrophic stage, are conserved in other Ustilago species (Hemetsberger et al. 2012; Redkar et al. 2015). These examples illustrate that pathogen effectors are not always lineage-specific but can be conserved even across multiple kingdoms of life.

Bacterial flagellin is a MAMP that is broadly conserved between species. However, within species polymorphisms in flagellin have been identified in which some versions are recognized and others are not (Cai et al. 2011; Clarke et al. 2013; Sun et al. 2006). This shows that MAMPs are not always conserved and can also mutate to avoid recognition. Therefore, MAMPs and effectors and, consequently, MTI and ETI cannot be strictly separated, but rather form a continuum (Thomma et al. 2011).

The zigzag model adequately describes the interaction of biotrophic pathogens with their hosts, as they are generally affected by immune responses that involve host cell death. However, necrotrophic pathogens that actively kill host cells to access nutrients actually benefit from cell death activated by immune receptors (Glazebrook 2005). This has been shown for the fungal pathogen Cochliobolus victoriae effector victorin that binds to TRXh5 which activates immune receptor LOV1, resulting in cell death that benefits the pathogen instead of stopping the pathogen (Lorang et al. 2012). Thus, although recognition of effectors of biotrophic and necrotrophic pathogens similarly leads to cell death, the outcome for the interaction is significantly different. Thus, the interaction between necrotrophic pathogens and their hosts is generally not well described by the zigzag model. 
Similarly, the zigzag model does not account for the interaction between plants and mutualistic microbes that use effectors as well (Plett and Martin 2015). Cell death does not benefit the 'desired' outcome of the interaction between plants and mutualists, and therefore the zigzag model does not accurately describe these interactions.

Examples such as those above have been the inspiration to propose an alternative model to describe plant-microbe interactions, in which a continuum between MAMPs and effectors is appreciated on the one hand, and similarly between MTI and ETI on the other hand. This alternative model is named the "the invasion model" (Cook et al. 2015), which states that any signal that reveals invasion is a so-called invasion pattern (IP) that can potentially be detected by the plant through invasion pattern receptors (IPRs), leading to an appropriate response (IP-triggered response; IPTR) that either supports or obstructs microbial colonization of the host.

\section{Population genomics to identify genomic regions that are associated with pathogenicity}

The ease and low cost of present-day genome sequencing (Faino and Thomma 2014) has made it possible to sequence pathogen populations instead of a single genome per species. This has led to new insights in genome and effector evolution. Certain regions of fungal pathogen genomes are enriched in effector genes. For example, in the genome of the asexual pathogen $V$. dahliae chromosomal rearrangements are associated with highly dynamic lineage-specific regions that are enriched for in planta-induced effector genes and active transposons (de Jonge et al. 2013; Faino et al. 2016). Also in the fungal Brassica pathogen Leptosphaeria maculans and the potato famine oomycete Phythophtora infestans it was shown that genepoor and repeat-rich regions of the genome are highly dynamic and enriched for in planta expressed effector genes, in contrast to gene-rich and repeat-poor regions of the genome (Dong et al. 2015). These observations illustrate the "the two-speed genome" model, in which effectors reside in fast evolving regions of the genome (Croll and McDonald 2012; Stukenbrock et al. 2010).

Various pathogens carry conditionally dispensable chromosomes (CDCs) that are enriched in effector genes. In the genome of the tomato pathogen Alternaria alternate a CDC contains all the genes responsible for AAL-toxin production (Akagi et al. 2009). More strikingly, in the genome of the tomato pathogen F. oxysporum f. sp. lycopersici a CDC contains all but one SIX (for secreted in xylem) effector genes (Ma et al. 2010; Schmidt et al. 2013; Van Dam et al. 2016), including FoAve1 (Chapter 3 , this thesis)(de Jonge et al. 2012). When this chromosome, which is also referred to as a pathogenicity chromosome, is transferred to a non-pathogenic isolate of $F$. oxysporum, this isolate gains the capacity to infect tomato (Ma et al. 2010). 


\section{Effector discovery based on motifs and similarity}

Many effectors have been identified based on their characteristics as small secreted cysteine-rich proteins. Depending on the (type of) organism, additional motifs have been associated with effectors, such as the RXLR or CRN (Crinkler) motif in Phytophthora species (Haas et al. 2009), MIMP (miniature impala) motif presence in the promotor of F. oxysporum effectors (Schmidt et al. 2013), lysin motif (LysM) in fungal effectors (de Jonge and Thomma 2009), the type-III secretion signal in effectors of gram-negative bacteria and the TAL (transcription activator-like) effector DNA binding motif in Xanthomonas species (Boch and Bonas 2010). However, for most pathogens effector motifs have not yet been identified and effectors often do not display sequence homology to other effectors, which makes it challenging to identify novel effectors. Comparison of the 3D-structure of two sequence-unrelated effector proteins of the fungal rice pathogen Magnaporthe oryzae revealed structural similarity (de Guillen et al. 2015). Subsequent structure-informed pattern searches identified novel effectors in $M$. oryzae and $M$. grisea that were named MAX (Magnaporthe Avrs and ToxB like) effectors (de Guillen et al. 2015). A similar approach to identify novel effectors can likely be applied to other pathogens as well (Franceschetti et al. 2017).

\section{Identification of effectors through random mutagenesis}

Since the majority of effector genes that have been identified thus far are unique and do not have homologs in other pathogens, an unbiased approach should be applied to identify them. Indeed, using random mutagenesis through Agrobacterium tumefaciens-mediated transformation (ATMT) followed by a screen for reduced virulence on the host plant has proven to be successful in identifying novel effectors and pathogenicity factors in fungal pathogens including for example F. oxysporum (Michielse et al. 2009), Colletotrichum graminicola (Munch et al. 2011) and Botrytis cinerea (Giesbert et al. 2012). In a random mutagenesis screen on $V$. dahliae using ATMT 900 mutants were generated that, after thorough virulence testing and sequence analysis, led to the identification of 58 candidate pathogenicity and effector genes. One of the candidate genes, VdNRS/ER, was functionally analyzed and led to the finding that rhamnose synthase activity is required for pathogenicity of V. dahliae (Chapter 7, this thesis) (Santhanam et al. 2017).

\section{Population genomics as a tool for effector identification}

Population genomics has contributed to the discovery of effectors that are recognized by host receptors. For example, by sequencing the genomes of 11 race 1 and race 2 strains of $V$. dahliae and subsequent RNA sequencing, the race 
1-specific Ave1 effector that is recognized by tomato immune receptor Ve1 was identified (de Jonge et al. 2012). A similar approach was used to identify the Avr5 effector of the tomato leaf mould fungus Cladosporium fulvum that is recognized by the tomato immune receptor Cf-5 (Mesarich et al. 2014). Finally, the F. oxysporum f. sp. melonis effector AvrFom2 that is recognized by melon immune receptor Fom2 was identified using a comparable approach (Schmidt et al. 2016). Potentially, this powerful strategy may be applied to identify potent virulence factors that are differentially present in other pathogen populations.

\section{Effectors define host specificity}

Population genomics has revealed that effectors may be responsible for host specificity of a pathogen. Within the species complex of $F$. oxysporum, the formae speciales indicates on which host plant an isolate is pathogenic. Formae speciales are generally polyphyletic and therefore hard to identify based on conserved genes. However, effectors identified in F. oxysporum isolates from various host plants group together based on the same formae speciales, providing an identification tool based on effector presence or absence (Lievens et al. 2009; Van Dam et al. 2016). However, it should be taken into account that effector presence alone is not always proof that an isolate is pathogenic as non-pathogenic Fusarium isolates where found that carry effector genes too (Jelinski et al. 2017). Also, comparative genomics between strains of the bacterium Ralstonia solanacearum that are pathogenic on different host plants revealed effector repertoires associated with a specific host (Ailloud et al. 2015). Finally, P. infestans and its close relative P. mirabilis that is pathogenic on Mirabilis jalapa share a homologous effector that is only effective on their corresponding plant host (Dong et al. 2014).

Gene and effector expansions and contractions are linked to the host range of a pathogen. In V. dahliae, NLP effector expansion has been associated with functional diversification (Santhanam et al. 2013; Zhou et al. 2012). Only a subset of the NLP effectors in V. dahliae is cytotoxic (typical for NLPs) while others are non-cytotoxic. Besides a role in virulence, NLP1 was also shown to be involved in asexual reproduction and vegetative growth. NLP1 and NLP2 are virulence factors on tomato and Arabidopsis, but not on cotton, and only NLP1 is required for virulence on Nicotiana benthamiana (Santhanam et al. 2013; Zhou et al. 2012). This suggests that NLP diversification might have contributed to host range expansion. A comparison of species in the genus Colletotrichum that have a broad host range with species that have a limited host range revealed that species with broad host ranges rely on secreted enzymes and NLPs, whereas narrow host range species have lost proteases and are more reliant on lineage-specific effectors (Baroncelli et al. 2016). The examples above illustrate that effector repertoires define the host 
(range) of a pathogen. Moreover, as effectors are important virulence factors of pathogens that are potentially recognized by related or unrelated plant species of the host, they can be exploited as tools to identify novel resistance sources.

\section{Effectors as tools for pathogen resistance breeding}

Traditional plant breeding is a tedious and time-consuming practice in which marker-assisted selection has significantly increased the speed and efficiency of the selection process. However, pathogen assays are still an essential aspect for the development and verification of markers. Pathogen assays have limitations since they require extensive knowledge of the pathogens life cycle and can often only be performed with a single species or race of a given pathogen as resistance is usually species- or even race- specific. Once effectors have been identified for a given pathogen, these effectors can be used to screen germplasm for novel recognition specificities, and thus new resistance sources (Vleeshouwers and Oliver 2014). This can be done by transient expression of effector genes in germplasm by Agrobacteriummediated expression or potato virus X (PVX) (Chapman et al. 1992; Janssen and Gardner 1990). In this manner, many effectors can be tested in parallel, circumventing the limitations of disease assays, while simultaneously providing the possibility of screening germplasm that is a non-host to the pathogen or the possibility of screening effectors of pathogens with a complex lifecycle.

Since many effectors are race- or even strain- specific it is not very efficient to use these in a germplasm screen for resistance. Recognition specificity towards effectors that are conserved in a population of pathogens, also referred to as core effectors, is far more valuable. In a population genomics study on 65 isolates of the cassava bacterial blight pathogen Xanthomonas axonopodis pv. manihotis 9 effectors were identified that are conserved in the population (Bart et al. 2012). In a similar approach, 13 conserved effectors were identified in 67 isolates that belong to three Xanthomonas species that are pathogenic on tomato and pepper (Potnis et al. 2011; Schwartz et al. 2015). Population genomics on strains of $V$. dahliae also revealed effectors that are conserved in the population (de Jonge et al. 2013). Identification and exploitation of $R$ genes that recognize these conserved effectors might potentially lead to durable pathogen resistance.

Knowledge of the effectors that are present in a given population can also aid in developing recognition specificity towards (a) certain effector(s). A single amino acid substitution can already broaden the specificity of an $R$ gene. This was shown for potato NB-LRR immune receptor R3a by performing a gain-of-recognition screen after random mutagenesis in which the recognition specificity was extended to isoforms of the recognized P. infestans effector Avr3A that are predominant in Phytophthora populations world-wide (Segretin et al. 2014). 12 is a NB-LRR immune receptor of 
tomato that confers resistance to $F$. oxysporum and partial resistance to $P$. infestans and shows high similarity to R3a (Giannakopoulou et al. 2015). Mutations that extended recognition in potato R3a were also mutated in tomato 12 which resulted in a broader and enhanced specificity to both P. infestans effector isoforms of Avr3a and F. oxysporum effector isoforms of Avr2 (Giannakopoulou et al. 2015). These examples illustrate that with relatively small adjustments the recognition specificity of an immune receptor can be extended. Potentially, this strategy can be exploited to increase the recognition specificity of Ve1 towards pathogens that secrete homologs of Ave1 that are poorly recognized.

Like 12 , other $R$ genes or combinations of genes have been described to provide resistance to two or more pathogens. Tomato immune receptor $\mathrm{Cf}-2$ guards Rcr3 that is targeted by effectors of the fungus $C$. fulvum and the nematode Globodera rostochiensis resulting in a defence response (Lozano-Torres et al. 2012). Thus, naturally occurring $R$ genes can be exploited that confer resistance to multiple pathogens.

In a map-based cloning approach combined with natural variation analysis in Arabidopsis a dual $R$ gene system consisting of the nucleotide binding, leucine-rich repeat (NLR) domain receptors RPS4-Ws and RRS1-Ws was identified that provides resistance to the fungus $C$. higginsianum, the bacteria Ralstonia solanacearum and Pseudomonas syringae pv. tomato (Deslandes et al. 2002; Narusaka et al. 2009). Other dual $R$ gene systems have been characterized as well, such as the rice NLRs RGA4 and RGA5 that interact to provide resistance to $M$. oryzae (Césari et al. 2014a). In these NLR pairs integrated domains (NLR-IDs) have been identified that serve as a bait for effectors to activate immunity as has been described in the integrated decoy hypothesis (Césari et al. 2014b). NLR-ID integration in plant immune receptors is broadly distributed in the plant kingdom (Kroj et al. 2016; Sarris et al. 2016) and analyses of grass genomes suggests the presence of NLR 'acceptor' genes that likely acquire these domains from elsewhere in the genome (Bailey et al. 2017). Potentially, modification or transfer of NLR-IDs to other NLRs can contribute to synthesizing novel $R$ genes (Kim et al. 2016; Rodriguez-Moreno et al. 2017).

Often $R$ genes are identified or characterized in wild species or model organisms and cannot easily be introduced into crops by crossing. However, interfamily transfer of receptor-like proteins (RLPs) and receptor kinases (RKs) has been quite successful. For example, the Arabidopsis thaliana receptor kinase EFR that recognizes bacterial elongation factor Tu (EF-Tu) was transferred to tomato (Solanum lycopersicum) providing broad bacterial resistance (Lacombe et al. 2010). Similarly, the A. thaliana receptor kinase LecRK-I.9 which provides resistance to $P$. infestans was functionally transferred to potato (S. tuberosum) (Bouwmeester et al. 2014). Interfamily transfer of NB-LRRs seems to be less efficient compared to transfer of RLPs and RKs. Perhaps, for NLRs it is more efficient to transfer genes from wild relatives into crops (Bent 2016). 


\section{Susceptibility genes as a source of resistance}

Resistance is often overcome within a couple of years after the introduction of an $R$ gene (Rouxel et al. 2003; Sprague et al. 2006a; Sprague et al. 2006b). It has been proposed that mutation of host components that are required for a pathogen to successfully colonize its host, also known as susceptibility (S) genes (Eckardt 2002), is a more durable strategy to provide pathogen resistance (McDonald and Linde 2002). An example of an $S$ gene is the Mlo gene that provides resistance to powdery mildew in many crops including monocots and dicots (Kusch and Panstruga 2017). In barley, a recessive mutant of this gene was introduced 70 years ago and is still providing resistance to all powdery mildews in the field (Jørgensen 1992). Effector targets can be mutated with the consequence that the corresponding effector becomes useless. However, $S$ genes have other functions in the plant as well. Therefore it should be taken into account that mutating an $S$ gene can have detrimental effects on the plant (van Schie and Takken 2014).

\section{Genome editing in crops}

Genome editing in crops has made considerable steps forward over the last few years. With synthetic nucleases such as zinc finger nucleases (ZFNs), transcription activator-like effector nucleases (TALENs) and Cas9 of the clustered regularly interspaced short palindromic repeats (CRISPR) system it is possible to knockout genes, delete or insert sequences without selection markers (Puchta 2017; Schiml and Puchta 2016). Arguably, CRISPR/Cas9 is the most efficient and flexible system to work with (Puchta 2017). Concerns about off-target mutations seem to be a problem of the past and efficiencies of $4-9 \%$ have been reached. The strength of the CRISPR/Cas9 system is that it is possible to make precise changes in a sequence without the introduction of foreign DNA and marker genes. This was elegantly shown by editing the genomes of maize and bread wheat (Liang et al. 2017; Svitashev et al. 2016). In tomato, CRISPR/Cas9 was used to inactivate the $S$ gene orthologue DMR6 (for downy mildew resistance 6) that was characterized in A. thaliana (Van Damme et al. 2008), resulting in resistance to Pseudomonas syringae, Phytophtora capsici and Xanthomonas species (de Toledo Thomazella et al. 2016). Similarly, a deletion generated in an Mlo orthologue by CRISPR/Cas9 in tomato resulted in the variety Tomelo which is resistant to the powdery mildew fungal pathogen Oidium neolycopersici (Nekrasov et al. 2017). For this variety it was shown with whole-genome sequencing that it does not carry any foreign DNA, does not have off-target mutations and carries a single deletion that is indistinguishable from naturally occurring mutations. Whether genome editing using CRISPR/Cas9 will skip regulation as a genetically modified organism (GMO) in the EU should become clear in the near future (Waltz 2016; Wolter and Puchta 2017). 


\section{Management strategies to prevent resistance breakdown}

Unfortunately, current agricultural practice is still based on planting vast areas of monocultures, often carrying only a single $R$ gene against a given pathogen, season-after-season. This puts a huge selection pressure on pathogen populations to overcome resistance. Resistance breakdown can occur within a few years after the introduction of an R gene (Rouxel et al. 2003; Sprague et al. 2006a; Sprague et al. 2006b).

$R$ gene stacking, the introduction of multiple $R$ genes in a single variety, significantly increases the hurdle for a pathogen to overcome resistance (McDonald and Linde 2002; Michelmore et al. 2013). $R$ gene stacking can be achieved by gene transfer or marker-assisted breeding (Dangl et al. 2013). Using a cisgenic approach, broad spectrum Rpi (for resistance against $P$. infestans) genes were stacked and introduced in the susceptible potato cultivar Désirée to provide durable resistance to $P$. infestans and were subsequently monitored in field trials in the Netherlands (Haverkort et al. 2016; Tan et al. 2010; Zhu et al. 2013; Zhu et al. 2012). Several combinations of Rpi genes provided resistance to $P$. infestans and spray applications were significantly reduced (Haverkort et al. 2016). With marker-assisted breeding multiple $R$ genes were stacked in soybean to provide durable resistance to Soybean mosaic virus (SMV)(Maroof et al. 2008) and in wheat to provide resistance to powdery mildew (Liu et al. 2000). Criteria for stacking genes should be taken into account to prevent rapid resistance breakdown. These include: the stacked $R$ genes should target the same pathogen species, they should be introduced as a tandem construct to prevent segregation, have similar levels of expression in the same tissue and should target species that are susceptible to at least two of the stacked $R$ genes (Gressel et al. 2017). Obviously, extensive knowledge on $R$ genes and their corresponding effectors is essential to make educated decisions on which genes to stack. $R$ gene catalogues are available for multiple crop-pathogen interactions including, for example, potato and P. infestans, and lettuce and Bremia lactucae (Parra et al. 2016; Vleeshouwers et al. 2011). Monitoring pathogen populations in the field is essential to know which effectors are present in the pathogen population and to know at an early time point whether resistance breakdown occurs (Howlett et al. 2015; Michelmore et al. 2013). Subsequently, $R$ genes should be selected that provide resistance to conserved core effectors present in a pathogen population to increase the durability of resistance (Dangl et al. 2013). In addition, stacks of different $R$ genes should be used either within a variety or between different varieties to provide distinct selection pressure and therefore increased durability (Chin and Wolfe 1984; Michelmore et al. 2013). Finally, different $R$ genes or $R$ gene stacks should be used in time and space to break the continuous selection pressure that is put on pathogens. The criteria described above will ultimately lead to durable broad spectrum resistance in crops (Figure 1). 


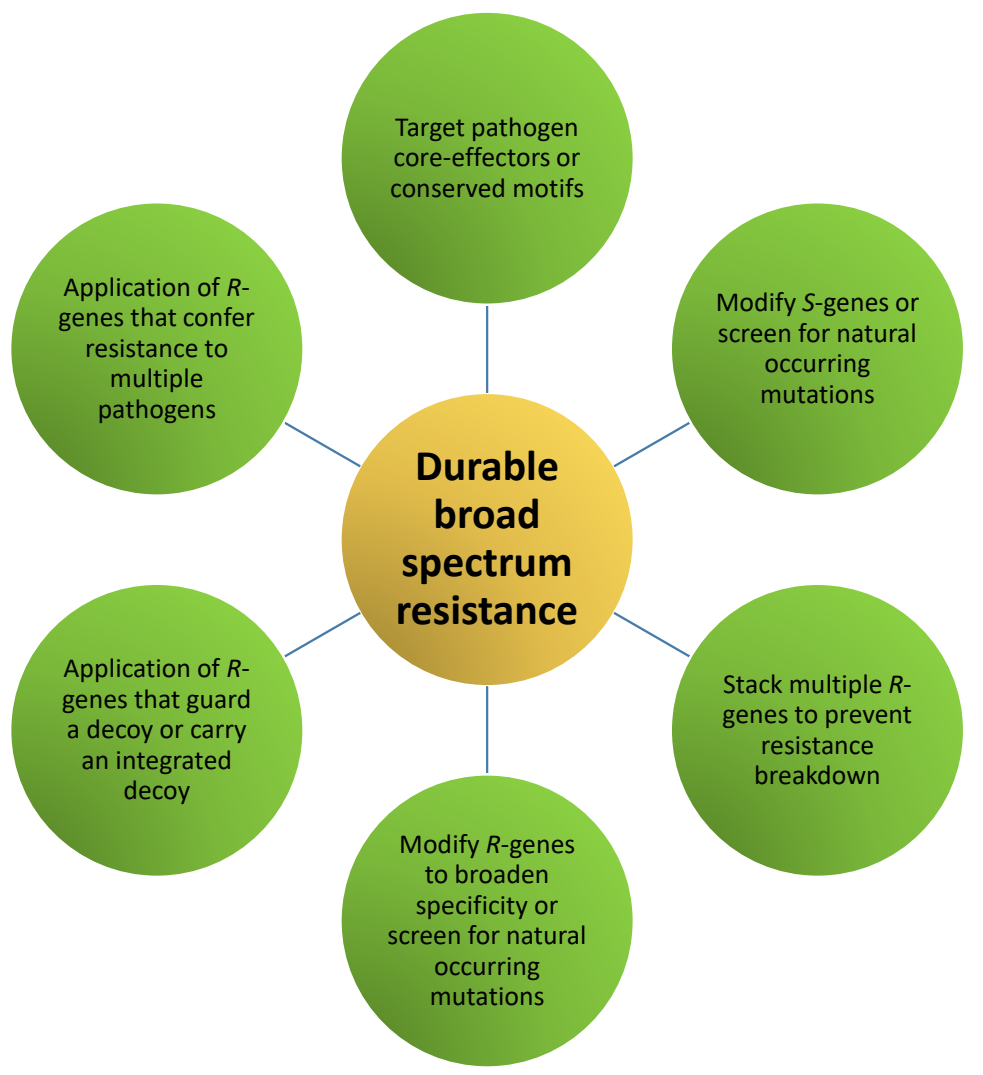

FIGURE 1 | Summarizing figure to visualize the many strategies that potentially lead to durable broad spectrum resistance in crops. 


\section{References}

Ailloud, F., Lowe, T., Cellier, G., Roche, D., Allen, C., and Prior, P. 2015. Comparative genomic analysis of Ralstonia solanacearum reveals candidate genes for host specificity. BMC Genomics 16:1.

Akagi, Y., Akamatsu, H., Otani, H., and Kodama, M. 2009. Horizontal chromosome transfer, a mechanism for the evolution and differentiation of a plant-pathogenic fungus. Eukaryotic cell 8:1732-1738.

Bailey, P. C., Dagdas, G., Baggs, E., Haerty, W., and Krasileva, K. V. 2017. Immune receptors with exogenous domain fusions form evolutionary hotspots in grass genomes. BioRxiv:100834.

Baroncelli, R., Amby, D. B., Zapparata, A., Sarrocco, S., Vannacci, G., Le Floch, G., Harrison, R. J., Holub, E., Sukno, S. A., and Sreenivasaprasad, S. 2016. Gene family expansions and contractions are associated with host range in plant pathogens of the genus Colletotrichum. BMC Genomics 17:555.

Bart, R., Cohn, M., Kassen, A., McCallum, E. J., Shybut, M., Petriello, A., Krasileva, K., Dahlbeck, D., Medina, C., and Alicai, T. 2012. High-throughput genomic sequencing of cassava bacterial blight strains identifies conserved effectors to target for durable resistance. Proceedings of the National Academy of Sciences 109:E1972-E1979.

Bent, A. 2016. Resistance from relatives. Nat Biotechnol 34:620-621.

Boch, J., and Bonas, U. 2010. Xanthomonas AvrBs3 family-type III effectors: discovery and function. Phytopathology 48:419.

Boller, T., and Felix, G. 2009. A renaissance of elicitors: perception of microbe-associated molecular patterns and danger signals by pattern-recognition receptors. Annu Rev Plant Biol 60:379-406.

Bouwmeester, K., Han, M., Blanco-Portales, R., Song, W., Weide, R., Guo, L.-Y., van der Vossen, E. A., and Govers, F. 2014. The Arabidopsis lectin receptor kinase LecRK-I. 9 enhances resistance to Phytophthora infestans in Solanaceous plants. Plant Biotechnology Journal 12:10-16.

Cai, R., Lewis, J., Yan, S., Liu, H., Clarke, C. R., Campanile, F., Almeida, N. F., Studholme, D. J., Lindeberg, M., and Schneider, D. 2011. The plant pathogen Pseudomonas syringae pv. tomato is genetically monomorphic and under strong selection to evade tomato immunity. PLoS Pathog 7:e1002130.

Césari, S., Bernoux, M., Moncuquet, P., Kroj, T., and Dodds, P. 2014b. A novel conserved mechanism for plant NLR protein pairs: the 'integrated decoy' hypothesis. Front Plant Sci 5.

Césari, S., Kanzaki, H., Fujiwara, T., Bernoux, M., Chalvon, V., Kawano, Y., Shimamoto, K., Dodds, P., Terauchi, R., and Kroj, T. 2014a. The NB-LRR proteins RGA4 and RGA5 interact functionally and physically to confer disease resistance. The EMBO journal 33:1941-1959.

Chapman, S., Kavanagh, T., and Baulcombe, D. 1992. Potato virus $X$ as a vector for gene expression in plants. Plant J 2:549-557.

Chin, K., and Wolfe, M. 1984. The spread of Erysiphe graminis f. sp. hordei in mixtures of barley varieties. Plant Pathol 33:89-100.

Chisholm, S. T., Coaker, G., Day, B., and Staskawicz, B. J. 2006. Host-microbe interactions: shaping the evolution of the plant immune response. Cell 124:803-814.

Clarke, C. R., Chinchilla, D., Hind, S. R., Taguchi, F., Miki, R., Ichinose, Y., Martin, G. B., Felix, G., and Vinatzer, B. A. 2013. Allelic variation in two distinct Pseudomonas syringae flagellin epitopes modulates the strength of plant immune responses but not bacterial motility. New Phytol 200:847860.

Cook, D. E., Mesarich, C. H., and Thomma, B. P. H. J. 2015. Understanding plant immunity as a surveillance system to detect invasion. Annu Rev Phytopathol.

Cooke, I. R., Jones, D., Bowen, J. K., Deng, C., Faou, P., Hall, N. E., Jayachandran, V., Liem, M., Taranto, A. P., Plummer, K. M., and Mathivanan, S. 2014. Proteogenomic analysis of the Venturia pirina (Pear Scab Fungus) secretome reveals potential effectors. J Proteome Res 13:3635-3644.

Croll, D., and McDonald, B. A. 2012. The accessory genome as a cradle for adaptive evolution in pathogens. PLoS Pathog 8:e1002608.

Dangl, J. L., Horvath, D. M., and Staskawicz, B. J. 2013. Pivoting the plant immune system from dissection to deployment. Science 341:746-751. 
de Guillen, K., Ortiz-Vallejo, D., Gracy, J., Fournier, E., Kroj, T., and Padilla, A. 2015. Structure analysis uncovers a highly diverse but structurally conserved effector family in phytopathogenic fungi. PLoS Pathog 11:e1005228.

de Jonge, R., and Thomma, B. P. 2009. Fungal LysM effectors: extinguishers of host immunity? Trends Microbiol 17:151-157.

de Jonge, R., Bolton, M. D., Kombrink, A., van den Berg, G. C., Yadeta, K. A., and Thomma, B. P. H. J. 2013. Extensive chromosomal reshuffling drives evolution of virulence in an asexual pathogen. Genome Res 23:1271-1282.

de Jonge, R., van Esse, H. P., Maruthachalam, K., Bolton, M. D., Santhanam, P., Saber, M. K., Zhang, Z., Usami, T., Lievens, B., Subbarao, K. V., and Thomma, B. P. H. J. 2012. Tomato immune receptor Ve1 recognizes effector of multiple fungal pathogens uncovered by genome and RNA sequencing. Proc Natl Acad Sci U S A 109:5110-5115.

de Toledo Thomazella, D. P., Brail, Q., Dahlbeck, D., and Staskawicz, B. J. 2016. CRISPR-Cas9 mediated mutagenesis of a DMR6 ortholog in tomato confers broad-spectrum disease resistance. BioRxiv:064824.

Deng, C. H., Plummer, K. M., Jones, D. A. B., Mesarich, C. H., Shiller, J., Taranto, A. P., Robinson, A. J., Kastner, P., Hall, N. E., Templeton, M. D., and Bowen, J. K. 2017. Comparative analysis of the predicted secretomes of Rosaceae scab pathogens Venturia inaequalis and $V$. pirina reveals expanded effector families and putative determinants of host range. BMC Genomics 18:339.

Deslandes, L., Olivier, J., Theulières, F., Hirsch, J., Feng, D. X., Bittner-Eddy, P., Beynon, J., and Marco, Y. 2002. Resistance to Ralstonia solanacearum in Arabidopsis thaliana is conferred by the recessive $R R S 1-R$ gene, a member of a novel family of resistance genes. Proceedings of the National Academy of Sciences 99:2404-2409.

Dong, S., Raffaele, S., and Kamoun, S. 2015. The two-speed genomes of filamentous pathogens: waltz with plants. Current Opinion in Genetics \& Development 35:57-65.

Dong, S., Stam, R., Cano, L. M., Song, J., Sklenar, J., Yoshida, K., Bozkurt, T. O., Oliva, R., Liu, Z., Tian, M., Win, J., Banfield, M. J., Jones, A. M., van der Hoorn, R. A., and Kamoun, S. 2014. Effector specialization in a lineage of the Irish potato famine pathogen. Science 343:552-555.

Eckardt, N. A. 2002. Plant disease susceptibility genes? Plant Cell 14:1983-1986.

Faino, L., and Thomma, B. P. 2014. Get your high-quality low-cost genome sequence. Trends Plant Sci 19:288-291.

Faino, L., Seidl, M. F., Shi-Kunne, X., Pauper, M., van den Berg, G. C., Wittenberg, A. H., and Thomma, B. P. 2016. Transposons passively and actively contribute to evolution of the two-speed genome of a fungal pathogen. Genome Res 26:1091-1100.

Ficarra, F. A., Grandellis, C., Garavaglia, B. S., Gottig, N., and Ottado, J. 2017. Bacterial and plant natriuretic peptides improve plant defense responses against pathogens. Mol Plant Pathol.

Flor, H. H. 1942. Inheritance of pathogenicity in Melampsora lini. Phytopathology 32:653-669.

Franceschetti, M., Maqbool, A., Jiménez-Dalmaroni, M. J., Pennington, H. G., Kamoun, S., and Banfield, M. J. 2017. Effectors of filamentous plant pathogens: commonalities amid diversity. Microbiology and Molecular Biology Reviews 81:e00066-00016.

Giannakopoulou, A., Steele, J. F., Segretin, M. E., Bozkurt, T. O., Zhou, J., Robatzek, S., Banfield, M. J., Pais, M., and Kamoun, S. 2015. Tomato 12 immune receptor can be engineered to confer partial resistance to the oomycete Phytophthora infestans in addition to the fungus Fusarium oxysporum. Mol. Plant Microbe Interact. 28:1316-1329.

Giesbert, S., Schumacher, J., Kupas, V., Espino, J., Segmuller, N., Haeuser-Hahn, I., Schreier, P. H., and Tudzynski, P. 2012. Identification of pathogenesis-associated genes by T-DNA-mediated insertional mutagenesis in Botrytis cinerea: a type 2A phosphoprotein phosphatase and an SPT3 transcription factor have significant impact on virulence. Mol. Plant Microbe Interact. 25:481-495.

Glazebrook, J. 2005. Contrasting mechanisms of defense against biotrophic and necrotrophic pathogens. Annu Rev Phytopathol 43:205-227. 
Gottig, N., Garavaglia, B. S., Daurelio, L. D., Valentine, A., Gehring, C., Orellano, E. G., and Ottado, J. 2008. Xanthomonas axonopodis pv. citri uses a plant natriuretic peptide-like protein to modify host homeostasis. Proc Natl Acad Sci U S A 105:18631-18636.

Gressel, J., Gassmann, A. J., and Owen, M. D. 2017. How well will stacked transgenic pest/herbicide resistances delay pests from evolving resistance? Pest Management Science 73:22-34.

Haas, B. J., Kamoun, S., Zody, M. C., Jiang, R. H., Handsaker, R. E., Cano, L. M., Grabherr, M., Kodira, C. D., Raffaele, S., and Torto-Alalibo, T. 2009. Genome sequence and analysis of the Irish potato famine pathogen Phytophthora infestans. Nature 461:393-398.

Haverkort, A. J., Boonekamp, P. M., Hutten, R., Jacobsen, E., Lotz, L. A. P., Kessel, G. J. T., Vossen, J. H., and Visser, R. G. F. 2016. Durable Late Blight Resistance in Potato Through Dynamic Varieties Obtained by Cisgenesis: Scientific and Societal Advances in the DuRPh Project. Potato Research 59:35-66.

Hemetsberger, C., Herrberger, C., Zechmann, B., Hillmer, M., and Doehlemann, G. 2012. The Ustilago maydis effector Pep1 suppresses plant immunity by inhibition of host peroxidase activity. PLoS Pathog 8:e1002684.

Howlett, B. J., Lowe, R. G., Marcroft, S. J., and van de Wouw, A. P. 2015. Evolution of virulence in fungal plant pathogens: exploiting fungal genomics to control plant disease. Mycologia 107:441-451.

Janssen, B.-J., and Gardner, R. C. 1990. Localized transient expression of GUS in leaf discs following cocultivation with Agrobacterium. Plant Mol Biol 14:61-72.

Jelinski, N., Broz, K., Jonkers, W., Ma, L.-J., and Kistler, H. C. 2017. Effector Gene Suites in Some Soil Isolates of Fusarium oxysporum Are not Sufficient Predictors of Vascular Wilt in Tomato. Phytopathology.

Jones, J. D., and Dangl, J. L. 2006. The plant immune system. Nature 444:323-329.

Jørgensen, J. H. 1992. Discovery, characterization and exploitation of Mlo powdery mildew resistance in barley. Pages 141-152 in: Breeding for disease resistance. Springer.

Keen, N. 1990. Gene-for-gene complementarity in plant-pathogen interactions. Annu Rev Gen 24:447463.

Kim, S. H., Qi, D., Ashfield, T., Helm, M., and Innes, R. W. 2016. Using decoys to expand the recognition specificity of a plant disease resistance protein. Science 351:684-687.

Kombrink, A., and Thomma, B. P. H. J. 2013. LysM effectors: secreted proteins supporting fungal life. PLoS Pathog 9:e1003769.

Kroj, T., Chanclud, E., Michel-Romiti, C., Grand, X., and Morel, J. B. 2016. Integration of decoy domains derived from protein targets of pathogen effectors into plant immune receptors is widespread. New Phytol 210:618-626.

Kusch, S., and Panstruga, R. 2017. mlo-Based Resistance: An Apparently Universal "Weapon" to Defeat Powdery Mildew Disease. Mol. Plant Microbe Interact. 30:179-189.

Lacombe, S., Rougon-Cardoso, A., Sherwood, E., Peeters, N., Dahlbeck, D., van Esse, H. P., Smoker, M., Rallapalli, G., Thomma, B. P. H. J., Staskawicz, B. J., Jones, J. D., and Zipfel, C. 2010. Interfamily transfer of a plant pattern-recognition receptor confers broad-spectrum bacterial resistance. Nat Biotechnol 28:365-369.

Liang, Z., Chen, K., Li, T., Zhang, Y., Wang, Y., Zhao, Q., Liu, J., Zhang, H., Liu, C., and Ran, Y. 2017. Efficient DNA-free genome editing of bread wheat using CRISPR/Cas9 ribonucleoprotein complexes. Nat Commun 8.

Lievens, B., Houterman, P. M., and Rep, M. 2009. Effector gene screening allows unambiguous identification of Fusarium oxysporum f. sp. lycopersici races and discrimination from other formae speciales. FEMS Microbiol Lett 300:201-215.

Liu, J., Liu, D., Tao, W., Li, W., Wang, S., Chen, P., Cheng, S., and Gao, D. 2000. Molecular markerfacilitated pyramiding of different genes for powdery mildew resistance in wheat. Plant Breeding 119:21-24. 
Lorang, J., Kidarsa, T., Bradford, C. S., Gilbert, B., Curtis, M., Tzeng, S. C., Maier, C. S., and Wolpert, T. J. 2012. Tricking the guard: exploiting plant defense for disease susceptibility. Science 338:659-662.

Lozano-Torres, J. L., Wilbers, R. H. P., Gawronski, P., Boshoven, J. C., Finkers-Tomczak, A., Cordewener, J. H. G., America, A. H. P., Overmars, H. A., Van 't Klooster, J. W., Baranowski, L., Sobczak, M., llyas, M., van der Hoorn, R. A. L., Schots, A., de Wit, P. J. G. M., Bakker, J., Goverse, A., and Smant, G. 2012. Dual disease resistance mediated by the immune receptor $C f-2$ in tomato requires a common virulence target of a fungus and a nematode. Proc Natl Acad Sci U S A 109:10119-10124.

Ma, L. J., van der Does, H. C., Borkovich, K. A., Coleman, J. J., Daboussi, M. J., Di Pietro, A., Dufresne, M., Freitag, M., Grabherr, M., Henrissat, B., Houterman, P. M., Kang, S., Shim, W. B., Woloshuk, C., Xie, X., Xu, J. R., Antoniw, J., Baker, S. E., Bluhm, B. H., Breakspear, A., Brown, D. W., Butchko, R. A., Chapman, S., Coulson, R., Coutinho, P. M., Danchin, E. G., Diener, A., Gale, L. R., Gardiner, D. M., Goff, S., Hammond-Kosack, K. E., Hilburn, K., Hua-Van, A., Jonkers, W., Kazan, K., Kodira, C. D., Koehrsen, M., Kumar, L., Lee, Y. H., Li, L., Manners, J. M., Miranda-Saavedra, D., Mukherjee, M., Park, G., Park, J., Park, S. Y., Proctor, R. H., Regev, A., Ruiz-Roldan, M. C., Sain, D., Sakthikumar, S., Sykes, S., Schwartz, D. C., Turgeon, B. G., Wapinski, I., Yoder, O., Young, S., Zeng, Q., Zhou, S., Galagan, J., Cuomo, C. A., Kistler, H. C., and Rep, M. 2010. Comparative genomics reveals mobile pathogenicity chromosomes in Fusarium. Nature 464:367-373.

Maroof, S., Jeong, S., Gunduz, I., Tucker, D., Buss, G., and Tolin, S. 2008. Pyramiding of soybean mosaic virus resistance genes by marker-assisted selection. Crop Science 48:517-526.

McDonald, B. A., and Linde, C. 2002. Pathogen population genetics, evolutionary potential, and durable resistance. Annu Rev Phytopathol 40:349-379.

Mesarich, C. H., Griffiths, S. A., van der Burgt, A., Okmen, B., Beenen, H. G., Etalo, D. W., Joosten, M. H., and de Wit, P. J. G. M. 2014. Transcriptome sequencing uncovers the Avr5 avirulence gene of the tomato leaf mold pathogen Cladosporium fulvum. Mol. Plant Microbe Interact. 27:846-857.

Michelmore, R. W., Christopoulou, M., and Caldwell, K. S. 2013. Impacts of resistance gene genetics, function, and evolution on a durable future. Annu Rev Phytopathol 51:291-319.

Michielse, C. B., van Wijk, R., Reijnen, L., Cornelissen, B. J., and Rep, M. 2009. Insight into the molecular requirements for pathogenicity of Fusarium oxysporum f. sp. lycopersici through large-scale insertional mutagenesis. Genome Biol 10:R4.

Munch, S., Ludwig, N., Floss, D. S., Sugui, J. A., Koszucka, A. M., Voll, L. M., Sonnewald, U., and Deising, H. B. 2011. Identification of virulence genes in the corn pathogen Colletotrichum graminicola by Agrobacterium tumefaciens-mediated transformation. Mol Plant Pathol 12:43-55.

Narusaka, M., Shirasu, K., Noutoshi, Y., Kubo, Y., Shiraishi, T., Iwabuchi, M., and Narusaka, Y. 2009. RRS1 and RPS4 provide a dual Resistance-gene system against fungal and bacterial pathogens. Plant $J$ 60:218-226.

Nekrasov, V., Wang, C., Win, J., Lanz, C., Weigel, D., and Kamoun, S. 2017. Rapid generation of a transgene-free powdery mildew resistant tomato by genome deletion. Scientific Reports 7:482.

Oome, S., Raaymakers, T. M., Cabral, A., Samwel, S., Böhm, H., Albert, I., Nürnberger, T., and Van den Ackerveken, G. 2014. Nep1-like proteins from three kingdoms of life act as a microbe-associated molecular pattern in Arabidopsis. Proc Natl Acad Sci U S A 111:16955-16960.

Parra, L., Maisonneuve, B., Lebeda, A., Schut, J., Christopoulou, M., Jeuken, M., McHale, L., Truco, M.J., Crute, I., and Michelmore, R. 2016. Rationalization of genes for resistance to Bremia lactucae. Euphytica 210:309-326.

Pemberton, C. L., and Salmond, G. P. 2004. The Nep1-like proteins-a growing family of microbial elicitors of plant necrosis. Mol Plant Pathol 5:353-359.

Plett, J. M., and Martin, F. 2015. Reconsidering mutualistic plant-fungal interactions through the lens of effector biology. Curr Opin Plant Biol 26:45-50.

Potnis, N., Minsavage, G., Smith, J. K., Hurlbert, J. C., Norman, D., Rodrigues, R., Stall, R. E., and Jones, J. B. 2011. Avirulence Proteins AvrBs7 from Xanthomonas gardneri and AvrBs1.1 from Xanthomonas euvesicatoria Contribute to a Novel Gene-for-Gene Interaction in Pepper. Mol. Plant Microbe Interact. 25:307-320. 
Puchta, H. 2017. Applying CRISPR/Cas for genome engineering in plants: the best is yet to come. Curr Opin Plant Biol 36:1-8.

Redkar, A., Villajuana-Bonequi, M., and Doehlemann, G. 2015. Conservation of the Ustilago maydis Effector See1 in related smuts. Plant Signal Behav 10:e1086855.

Rodriguez-Moreno, L., Song, Y., and Thomma, B. P. H. J. 2017. Transfer and engineering of immune receptors to improve recognition capacities in crops. Curr Opin Plant Biol 38:42-49.

Rouxel, T., Penaud, A., Pinochet, X., Brun, H., Gout, L., Delourme, R., Schmit, J., and Balesdent, M.H. 2003. A 10-year survey of populations of Leptosphaeria maculans in France indicates a rapid adaptation towards the RIm1 resistance gene of oilseed rape. Eur J Plant Pathol 109:871-881.

Rovenich, H., Boshoven, J. C., and Thomma, B. P. H. J. 2014. Filamentous pathogen effector functions: of pathogens, hosts and microbiomes. Curr Opin Plant Biol 20:96-103.

Santhanam, P., van Esse, H. P., Albert, I., Faino, L., Nurnberger, T., and Thomma, B. P. H. J. 2013. Evidence for functional diversification within a fungal NEP1-like protein family. Mol. Plant Microbe Interact. 26:278-286.

Santhanam, P., Boshoven, J. C., Salas, O., Bowler, K., Islam, M. T., Saber, M. K., van den Berg, G., BarPeled, M., and Thomma, B. P. 2017. Rhamnose synthase activity is required for pathogenicity of the vascular wilt fungus Verticillium dahliae. Mol Plant Pathol 18:347-362.

Sarris, P. F., Cevik, V., Dagdas, G., Jones, J. D., and Krasileva, K. V. 2016. Comparative analysis of plant immune receptor architectures uncovers host proteins likely targeted by pathogens. BMC biology 14:8.

Schiml, S., and Puchta, H. 2016. Revolutionizing plant biology: multiple ways of genome engineering by CRISPR/Cas. Plant methods 12:8.

Schmidt, S. M., Lukasiewicz, J., Farrer, R., Dam, P., Bertoldo, C., and Rep, M. 2016. Comparative genomics of Fusarium oxysporum f. sp. melonis reveals the secreted protein recognized by the Fom2 resistance gene in melon. New Phytol 209:307-318.

Schmidt, S. M., Houterman, P. M., Schreiver, I., Ma, L., Amyotte, S., Chellappan, B., Boeren, S., Takken, F. L., and Rep, M. 2013. MITEs in the promoters of effector genes allow prediction of novel virulence genes in Fusarium oxysporum. BMC Genomics 14:119.

Schwartz, A. R., Potnis, N., Timilsina, S., Wilson, M., Patané, J., Martins, J., Minsavage, G. V., Dahlbeck, D., Akhunova, A., and Almeida, N. 2015. Phylogenomics of Xanthomonas field strains infecting pepper and tomato reveals diversity in effector repertoires and identifies determinants of host specificity. Frontiers in Microbiology 6:535.

Segretin, M. E., Pais, M., Franceschetti, M., Chaparro-Garcia, A., Bos, J. I. B., Banfield, M. J., and Kamoun, S. 2014. Single Amino Acid Mutations in the Potato Immune Receptor R3a Expand Response to Phytophthora Effectors. Mol. Plant Microbe Interact. 27:624-637.

Song, Y., Zhang, Z., Boshoven, J., Rovenich, H., Seidl, M., Jakse, J., Maruthachalam, K., Liu, C.-M., Subbarao, K., and Javornik, B. 2017. Tomato immune receptor Ve1 recognizes surface-exposed co-localized N-and C-termini of Verticillium dahliae effector Ave1. BioRxiv:103473.

Sprague, S., Marcroft, S., Hayden, H., and Howlett, B. 2006a. Major gene resistance to blackleg in Brassica napus overcome within three years of commercial production in southeastern Australia. Plant Dis 90:190-198.

Sprague, S. J., Balesdent, M.-H., Brun, H., Hayden, H. L., Marcroft, S. J., Pinochet, X., Rouxel, T., and Howlett, B. J. 2006b. Major gene resistance in Brassica napus (oilseed rape) is overcome by changes in virulence of populations of Leptosphaeria maculans in France and Australia. Eur J Plant Pathol 114:33-40.

Stukenbrock, E. H., Jorgensen, F. G., Zala, M., Hansen, T. T., McDonald, B. A., and Schierup, M. H. 2010. Whole-genome and chromosome evolution associated with host adaptation and speciation of the wheat pathogen Mycosphaerella graminicola. PLoS Genet 6:e1001189. 
Sun, W., Dunning, F. M., Pfund, C., Weingarten, R., and Bent, A. F. 2006. Within-species flagellin polymorphism in Xanthomonas campestris pv campestris and its impact on elicitation of Arabidopsis FLAGELLIN SENSING2-dependent defenses. Plant Cell 18:764-779.

Svitashev, S., Schwartz, C., Lenderts, B., Young, J. K., and Cigan, A. M. 2016. Genome editing in maize directed by CRISPR-Cas9 ribonucleoprotein complexes. Nat Commun 7:13274.

Tan, M. Y. A., Hutten, R. C. B., Visser, R. G. F., and van Eck, H. J. 2010. The effect of pyramiding Phytophthora infestans resistance genes R Pi-mcd1 and R Pi-ber in potato. Theor Appl Genet 121:117-125.

Thomma, B. P. H. J., Nurnberger, T., and Joosten, M. H. A. J. 2011. Of PAMPs and effectors: The blurred PTI-ETI dichotomy. Plant Cell 23:4-15.

Van Dam, P., Fokkens, L., Schmidt, S. M., Linmans, J. H., Kistler, H. C., Ma, L. J., and Rep, M. 2016. Effector profiles distinguish formae speciales of Fusarium oxysporum. Environmental Microbiology.

Van Damme, M., Huibers, R. P., Elberse, J., and Van den Ackerveken, G. 2008. Arabidopsis DMR6 encodes a putative 2OG-Fe (II) oxygenase that is defense-associated but required for susceptibility to downy mildew. Plant J 54:785-793.

Van der Biezen, E. A., and Jones, J. D. 1998. Plant disease-resistance proteins and the gene-for-gene concept. Trends Biochem Sci 23:454-456.

van Schie, C. C., and Takken, F. L. 2014. Susceptibility genes 101: how to be a good host. Annu Rev Phytopathol 52:551-581.

Vleeshouwers, V. G., and Oliver, R. P. 2014. Effectors as tools in disease resistance breeding against biotrophic, hemibiotrophic, and necrotrophic plant pathogens. Mol. Plant Microbe Interact. 27:196-206.

Vleeshouwers, V. G., Raffaele, S., Vossen, J. H., Champouret, N., Oliva, R., Segretin, M. E., Rietman, H., Cano, L. M., Lokossou, A., and Kessel, G. 2011. Understanding and exploiting late blight resistance in the age of effectors. Annu Rev Phytopathol 49:507-531.

Waltz, E. 2016. CRISPR-edited crops free to enter market, skip regulation. Nature Publishing Group.

Wolter, F., and Puchta, H. 2017. Knocking out consumer concerns and regulator's rules: efficient use of CRISPR/Cas ribonucleoprotein complexes for genome editing in cereals. Genome Biol 18:43.

Zhou, B. J., Jia, P. S., Gao, F., and Guo, H. S. 2012. Molecular characterization and functional analysis of a necrosis- and ethylene-inducing, protein-encoding gene family from Verticillium dahliae. Mol. Plant Microbe Interact. 25:964-975.

Zhu, S., Li, Y., Vossen, J. H., Visser, R. G., and Jacobsen, E. 2012. Functional stacking of three resistance genes against Phytophthora infestans in potato. Transgenic research 21:89-99.

Zhu, S., Duwal, A., Su, Q., Vossen, J. H., Visser, R. G., and Jacobsen, E. 2013. Vector integration in triple $R$ gene transformants and the clustered inheritance of resistance against potato late blight. Transgenic research 22:315-325. 
Summary

Acknowledgements

About the author

List of publications

Overview of training activities 



\section{Summary}

Disease resistance in crops is an important aspect of securing global food security. Resistant plants carry immune receptors that sense pathogen invasion often through the recognition of important pathogen virulence factors, known as effectors. Thus, identification and characterization of effectors is important for the fundamental understanding of virulence mechanisms and to aid in resistance breeding. In Chapter 1, the motivation for selecting the plant pathogen Verticillium dahliae as a study object is explained. The main objectives and research questions are described and an outline of the thesis is provided. Chapter 2 reviews the various roles of effectors of filamentous plant pathogens and explains that effectors can basically deregulate any step of plant immunity in any cellular compartment. Besides in plant pathogens, endophytes and mutualistic organisms carry effectors that suppress host immunity as well. In addition, effectors have been identified in saprophytes and other non-pathogenic plant-associated microbes and are proposed to act in microbial competition and cooperation. We propose a role for effectors in shaping the plant microbiome and argue that it is important for a microbe to rapidly diversify its effector repertoire to establish a microbial niche.

The genus Verticillium contains soil-borne fungal pathogens causing Verticillium wilts in a wide range of host plants. The most effective means of controlling these pathogens is genetic disease resistance. The tomato immune receptor Ve1 provides resistance to race $1 \mathrm{~V}$. dahliae strains that carry Ave1 (VdAve1). Previously, it was shown that $V d A v e 1$ contributes to the virulence of $V$. dahliae and that VdAve1 is the effector that is recognized by Ve1. Homologs of $V d A v e 1$ were mostly found in plants and in some plant pathogens, including the fungi Fusarium oxysporum f. sp. lycopersici (FoAve1), Colletotrichum higginsianum (ChAve1), Cercospora beticola (CbAve1) and the bacterium Xanthomonas axonopodis (XacPNP). In Chapter 3 we analysed the role of the various Ave1 homologs in fungal virulence. Targeted Ave1 deletions were generated in F. oxysporum, C. beticola and C. higginsianum and we showed that FoAve1 and CbAve1 are virulence factors. This is in contrast to ChAve1, which does not contribute to fungal virulence on Arabidopsis thaliana plants, likely because this gene is not expressed during infection. In a complementation experiment we found that none of the fungal Ave1 homologs can restore virulence in a $V$. dahliae Ave1 deletion strain. Therefore we conclude that VdAve1 on the one hand and FoAve1, CbAve1, ChAve1 and XacPNP on the other hand, act in different manners. These homologs are differentially recognized by $\mathrm{Ve} 1$, with the most efficient recognition of $\mathrm{VdAve1}$, and little to no recognition of ChAve1 and XacPNP. In Chapter $\mathbf{4}$ we show that C-terminal GFP-tagging of VdAve1 resulted in loss of recognition by $V e 1$, suggesting that exposure of the C-terminus of VdAve1 is essential for recognition by Ve1. Truncations of VdAve1, 
and domain swaps between VdAve1 and XacPNP, narrowed down the sequence that is required for $\mathrm{Ve} 1$ recognition to the $\mathrm{C}$-terminal nine amino acids. Site-directed mutagenesis was performed to analyse the contribution of individual amino acid residues to recognition by $V e 1$, revealing the importance of five amino acids from Val127 to Phe131. Nevertheless, fusions of the VdAve1 C-terminal nine amino acids to GFP or to the Cladosporium fulvum effector Avr9 showed that this sequence is not sufficient to activate Ve1-mediated immunity. Finally, with a complementation experiment in $V$. dahliae we confirmed that the $\mathrm{C}$-terminal nine amino acids of Ave 1 are required, but not sufficient, to activate Ve1-mediated immunity. Chapter $\mathbf{5}$ describes the identification of an expanded family of Ave1 homologs in the apple and pear scab pathogens Venturia inaequalis and V. pirina, respectively. We show that tomato immune receptor $V e 1$ is unable to recognize Ave1 homologs derived from $V$. inaequalis and $V$. pirina. Transcriptome analysis revealed that $V$. inaequalis ViAve1-1, ViAve1-5 and ViAve1-9 are expressed in planta and that these homologs are unable to complement a $V$. dahliae $V d A v e 1$ deletion strain, suggesting that these homologs have different functions than V. dahliae VdAve1.

In Chapter 6, a gene with high similarity to VdAve1 was identified in V. dahliae strain JR2 as an Ave1-like (Ave1L). Subsequently, we identified a total of five alleles (Ave1L1 to Ave1L5) in the V. dahliae population. While some of the V. dahliae strains do not have Ave $1 L$, other strains have one of the five Ave $1 L$ alleles in presence or absence of Ave1 itself. Some of the Ave $1 \mathrm{~L}$ alleles contain multiple single nucleotide polymorphisms, some of which result in a premature stop codon. In addition, one of the alleles contains a retrotransposon insertion. These observations suggest that Ave1L experienced significant selection pressure, potentially to evade recognition by an immune receptor. Thus, we attempted to predict the common progenitor sequence of the five Ave1L alleles. However, we show that neither of the Ave1L alleles, nor the predicted progenitor, are recognized by tomato immune receptor Ve2 that shows high homology to Ve1. We furthermore show that Ave1L2 is expressed during host colonization, but does not seem to contribute to virulence on tomato. Since $V$. dahliae has a wide host range, Ave $1 L$ potentially contributes to virulence on other host plants than tomato where it may have been recognized by an immune receptor that drove its divergence.

In Chapter 7 random mutagenesis was performed on the $V$. dahliae genome, followed by a pathogenicity screen to identify genes that are important for host invasion. This resulted in the identification of 58 novel candidate genes. We focused on one of these candidates and showed that deletion of the VdNRS/ER gene from the $V$. dahliae genome results in complete loss of pathogenicity on tomato and $N$. benthamiana plants, whereas vegetative growth and sporulation are not affected. 
We demonstrate that VdNRS/ER is a functional enzyme in the biosynthesis of UDPrhamnose, and further analysis revealed that VdNRS/ER deletion strains are impaired in colonization of tomato roots. Collectively, our results demonstrate that rhamnose, although only being a minor cell wall component, is essential for pathogenicity of V. dahliae.

Chapter $\mathbf{8}$ is the general discussion and describes how the knowledge of pathogen effectors can be used as a tool to establish durable broad spectrum resistance. 



\section{Acknowledgements}

Here I am finishing the last part of my PhD thesis. The last part that is written, but often the first thing that people read when they open a $\mathrm{PhD}$ thesis. I guess it is because the acknowledgements show the personal side of the story. It is not possible to finish a PhD thesis on your own but it involves many people including your supervisor, colleagues, friends and family. Here I would like to take the opportunity to thank everybody involved.

I have mixed feelings on finishing the book but I feel mostly proud and relieved that it is done. Without my promotor Bart it would not have happened. I met Bart already in 2008, for my Hbo internship in the Verticillium group. Your style of supervision, your enthusiasm and the nice atmosphere in the group convinced me already then to keep my eyes open for a PhD position in your group. After obtaining my MSc degree I joined the Verticillium group as a PhD candidate. Bart, I am very happy you gave me the opportunity to start a project under your supervision. I enjoyed the freedom you gave me to find my own way in the project. Your office door was always open. Whenever I got stuck or lost in a situation you were able to guide me through it without giving me the answers. I am grateful for the many discussions we had about the project and the fast corrections and feedback I got on my manuscripts.

Pierre, my second promotor, thank you for the discussions in the beginning of the project and for helping me with the formalities at the end of the project. I feel honored to be your last PhD candidate.

I would like to thank the people in the Verticillium group who directly or indirectly helped me to get the experiments done in this thesis. Ronnie, for providing the data and material to initiate the project. Peter, for providing plasmids and constructs and showing me how to make good photos for publications. Partha, for teaching me how to transform Verticillium dahliae. Zhao and Yin, for providing strains and showing me how to do Agrobacterium infiltrations. Malaika, for helping me with the Cercospora beticola work. Eduardo, for all the qPCRs you run. Grardy, for teaching me how the Verticillium lab is operating and for all the help. Dirk Jan, for the help you gave me with plasmids and constructs. Michael, Luigi and Xiaoqian, for helping me with the many questions I had regarding evolution, genomes, rearrangements, phylogenetic trees, alignments, blasts and other bioinformatics related questions. David and Andrea, for the many discussions we had in the lab. Jinling, Luis and Jasper, and all other Verticillium lab members thank you for the nice atmosphere in the lab. I also thank you all for the nice discussions during the coffee brakes and the time we spend together. 
Then, I want to thank Jan, Francine and Matthieu for your input in the project. Klaas, Thomas, Daniela, Carl, Scott and Mansoor, thank you for all the useful suggestions and ideas but also the nice talks and times together. Thank you Ali, Rob, Grardy, Ester, Laurens and Natalie for making life easier in the lab, You made sure that the lab was up and running and that materials were available.

I would like to thank all the students that helped me with my projects. Jelle, Israel, Dorien, Saskia and Pascal without you it would have been a lot more work. Thank you Bert Essenstam and Pauline Sanderson at Unifarm for taking such good care of the plants in the greenhouse.

Dear Hanna, it was great to work with you on the same project. We had many workrelated and also work-unrelated discussions and you made me feel that I was not struggling alone $:-$. Our companionship gave me the positive energy to continue when things got difficult. Without you, these years would not have been the same. Apart from the PhD, I am happy we got the chance to become so close to and share beautiful moments together with you and Ale. I cherish all of them and I know that Iliana, Christof and Milo also do. I wish you all the best for the coming weeks and I am looking forward to meet the little one.

Mireille, I think we have managed to discuss almost everything, from experimental setup to family life. You were always in for a chat in the lab or at the coffee table. The talks were a nice distraction from aal the lab work and writing. I am convinced that without you my PhD time would have been a lot more boring and much more challenging. Thank you for being my paranymph.

Anjaatje, as I used to reply when you called me either Jordietje or Gordi, we have managed to built up a lot of memories in the past few years; skiing together with the Verticillium group in Winterberg after our meeting in Marbourg; visiting San Francisco before the Fungal Genetics conference in California. I really missed your presence in the lab when you moved to Zurich for your Postdoc. Thank you for the talks, dinners, drinks during my PhD. I am happy we keep contact and we always have things to share and talk about. Being my paranymph will be one more thing to remember $(:)$

Thanks to my Bejo colleagues for the support and talks during the breaks and lunch walks.

I would like to thank all my friends in Wageningen (few of you still there) during my studies which I am proud to still call friends. Without you it would not have been fun. Thank you Bart, Alejandro, Hanna, Aniek, Melchior, Andrius, Vaida, Abel, Maaike, Pavlo, Celine, Luigi, Alice, Bram, Anne, Xiaogian, Tim, Bram O, Sandra, Leandre, Johanna, Victoria, Tulipan, Alexander, Jule, Peter, Lorena, Jeroen, Wouter and my friends in Athens Gianni, loanna and Eleni. 
Thanks to my Greek family Dimitri, Maria, Stefano and Christina for supporting me. You always make me feel welcome and loved.

Many thanks to my parents Bert en Margreet for supporting me in all the years. I could not have done this without the confidence you had in me and the financial support you provided. I will always be very grateful for the opportunity you gave me to develop myself into the young professional I am now. Margreet, thank you for all the days you came to help in the house the last two years. Without your help I could not have finished this book.

Then last, but certainly not least, I want to thank my wife lliana. I know it has been a tough time for you too. Thank you so much for your support and patience when I spent hours writing in the evenings and weekends. Thank you for taking care of everything else so that I wouldn't have to stress. Thank you for listening to all my frustration and desperation and for the confidence you show in me. Thank you for your love and care and for the lovely family we have together. Christof and Philip are beautiful boys that have put things in perspective for me. Without the three of you I would not have been able to write this thesis. 


\section{About the author}

Jordi C. Boshoven was born in Alphen aan den Rijn, The Netherlands, on April 1 ${ }^{\text {st }} 1986$. At the age of 5 he moved to Maasdijk where he went to the PC Basisschool Het Kompas. His secondary education was performed at the Interconfessionele Scholengroep Westland (ISW) in Naaldwijk. During his holidays Jordi was working in the company of his uncle that is growing freesias in the greenhouse. In 2002, he went to the ROC Mondriaan in Delft where he was educated at

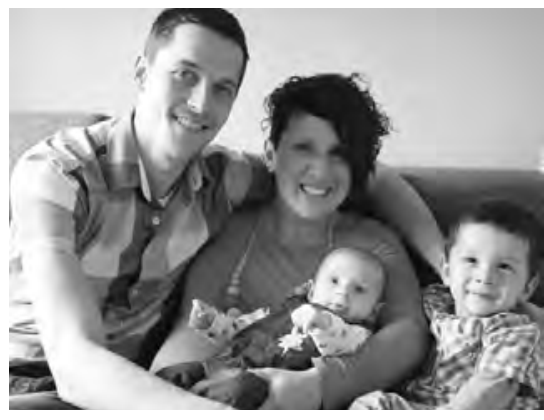
Mbo level with a specialization in Botany to work in a laboratory. His first internship was performed at Rijk Zwaan in De Lier under the supervision of Theresia van Luijk where he studied seed pathology. His second internship was performed at Groen Agro Control in Delfgauw under the supervision of Dr. Adriaan Vermunt and Laurens Hanemaaijer where he studied the molecular detection of plant pathogens in diagnostic samples.

In 2006, he continued his studies at Hbo level at the Hogeschool van Arnhem en Nijmegen (HAN) in Nijmegen where he studied Plant Biotechnology. During his education in Nijmegen he performed a minor, an internship and a thesis at Wageningen University. His internship was performed at the Laboratory of Phytopathology under the supervision of Prof. Dr. Bart Thomma where he studied plant recognition of the human bacterial pathogen Staphylococcus aureus. His thesis was performed in the Laboratory of Nematology under the supervision of Dr. Jose Lozano-Torres where he studied the recognition of the nematode effector Gr-VAP-1 by the tomato immune receptor Cf- 2 that resulted in a publication in the scientific journal Proceedings of the National Academy of Sciences of the United States of America.

After his studies in Nijmegen he moved to Wageningen in 2009 to do a MSc in Plant Biotechnology with the specialization Breeding and Pathology at Wageningen University. He performed his MSc internship in the group of Prof. Dr. Brian Staskawicz at the University of California in Berkley, USA under the supervision of Dr. Sandra Goritschnig where he studied the recognition of the oomycete Hyaloperonospora arabidopsidis effector ATR1 by the Arabidopsis immune receptor RPP1. His MSc thesis was performed at the PlantMicrobe Interactions group at Utrecht University under the supervision of Dr. Dieuwertje van der Does where he studied the jasmonic acid and salicylic acid plant defense signaling pathways in Arabidopsis.

After obtaining his MSc degree he started in 2012 as a PhD candidate in the Laboratory of Phytopathology under the supervision of Prof. Dr. Bart Thomma. He studied the virulence and recognition of Verticillium dahliae effector Ave1 homologs that resulted in the publication of this $\mathrm{PhD}$ thesis. During his $\mathrm{PhD}$ he organized several activities as a member of the PhD council of the graduate school of Experimental Plant Sciences (EPS). Since 2016 Jordi is working as a researcher phytopathology at Bejo Zaden in Warmenhuizen and he lives with his wife and two children in Koedijk. 


\section{List of publications}

Lozano-Torres, J. L., Wilbers, R. H. P., Gawronski, P., Boshoven, J. C., Finkers-Tomczak, A., Cordewener, J. H. G., America, A. H. P., Overmars, H. A., Van 't Klooster, J. W., Baranowski, L., Sobczak, M., llyas, M., van der Hoorn, R. A. L., Schots, A., de Wit, P. J. G. M., Bakker, J., Goverse, A., and Smant, G. 2012. Dual disease resistance mediated by the immune receptor $\mathrm{Cf}-2$ in tomato requires a common virulence target of a fungus and a nematode. Proc Natl Acad Sci U S A 109:10119-10124.

Rovenich, H., Boshoven, J. C., and Thomma, B. P. H. J. 2014. Filamentous pathogen effector functions: of pathogens, hosts and microbiomes. Curr Opin Plant Biol 20:96-103.

Santhanam, P., Boshoven, J. C., Salas, O., Bowler, K., Islam, M. T., Saber, M. K., van den Berg, G., Bar-Peled, M., and Thomma, B. P. 2017. Rhamnose synthase activity is required for pathogenicity of the vascular wilt fungus Verticillium dahliae. Mol Plant Pathol 18:347-362.

Song, Y., Zhang, Z., Boshoven, J. C., Rovenich, H., Seidl, M., Jakse, J., Maruthachalam, K., Liu, C.-M., Subbarao, K., and Javornik, B. 2017b. Tomato immune receptor Ve1 recognizes surface-exposed co-localized $\mathrm{N}$-and C-termini of Verticillium dahliae effector Ave1. BioRxiv:103473. 


\section{Education Statement of the Graduate School}

\section{Experimental Plant Sciences}

Issued to:

Date:

Group:

University:

\author{
Jordi C. Boshoven
}

10 November 2017

Laboratory of Phytopathology

Wageningen University \& Research

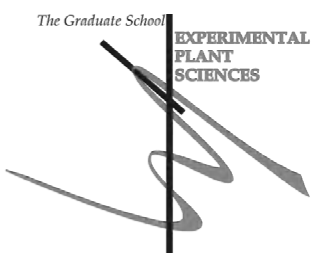

1) Start-up phase

date

- First presentation of your project

Title: The role of Verticillium dahliae Ave1

- Writing or rewriting a project proposal

Title: The role of Verticillium dahliae Ave1 in fungal virulence and its recognition by the tomato Ve1 immune receptor

- Writing a review or book chapter

Title: Filamentous Pathogen Effector Functions: Of Pathogens, Hosts and the Microbiome, Current Opinion in Plant Biology 20, 2014, 96-103; DOI: 10.1016/j.pbi.2014.05.001

- MSc courses

\section{Laboratory use of isotopes}

\section{Subtotal Start-up Phase}

8.5 credits* $^{*}$

\section{2) Scientific Exposure \\ - EPS PhD student days}

date

EPS PhD student day, Amsterdam, NL

30 Nov 2012

5th European Plant Science Retreat, Ghent University, Ghent, Belgium

23-26 Jul 2013

EPS PhD student day, Leiden, NL

29 Nov 2013

6th European Plant Science Retreat, UvA, Amsterdam, NI

01-04 Jul 2014

EPS PhD student day (GET2GETHER) Soest, NL

\section{- EPS theme symposia}

EPS Theme 2: Interactions between Plants and Biotic Agents \& Willie

10 Feb 2012

Commelin Scholten Day, Wageningen, NL

EPS Theme 2: Interactions between Plants and Biotic Agents \& Willie

24 Jan 2013

Commelin Scholten Day, Utrecht, NL

EPS Theme 2: Interactions between Plants and Biotic Agents \& Willie

Commelin Scholten Day, Amsterdam, NL

25 Feb 2014

EPS Theme 2: Interactions between Plants and Biotic Agents \& Willie

20 Feb 2015

Commelin Scholten Day, Utrecht, NL

- National meetings (e.g. Lunteren days) and other National Platforms

Annual meeting 'Experimental Plant Sciences', Lunteren, NL

02-03 Apr 2012

Annual meeting 'Experimental Plant Sciences', Lunteren, NL

22-23 Apr 2013

Annual meeting 'Experimental Plant Sciences', Lunteren, NL

14-15 Apr 2014

Annual meeting 'Experimental Plant Sciences', Lunteren, NL

13 Apr 2015 


\section{Seminars (series), workshops and symposia}

Workshops:

KLV speedreading course

08 Feb 2012

Lunchlezing "Initiatief nemen en assertiviteit - Hoe assertief kun je zijn?"

24 Sep 2014

Lunchlezing "Van spanning naar ontspanning, spelen met de dialoog"

18 Nov 2015

Seminars:

Monique van Oers 'Insect viruses and their applications'

23 Feb 2012

Ralph Panstruga 'Comparative pathogenomics of powdery mildew fungi'.

04 Dec 2012

Andrew Sugden (Editor of Science magazine) 'Demystifying Science'

08 Feb 2013

Detleif Weigel 'Origin and consequence of genetic and epigenetic variation'

27 Feb 2013

Kazuki Saito 'Metabolomics-based functional genomics - from Arabidopsis to

08 Apr 2013

crops and medicinal plants'

Brian Staskawicz "Effector-Targeted Breeding for Durable Disease Control of

Xanthomonas diseases in Tomato and Cassava"

21 May 2013

Pieter Dorrestein "A "GoogleMAP"-type molecular view of microbes - from culture to people"

Hanhui Kuang "Using the Nicotiana-TMV system to study resistance gene evolution and plant genome stability"

David Weller 'Soilborne Pathogens and their Natural Biocontrol Agents in

22 Aug 2013

Cereal-Based Production Systems"

Ross Mann "Endophytes in agriculture - evaluating their application via metabolomics and genomics"

Jos Raaijmakers 'Back to the roots: exploring and exploiting the plant microbiome'

Plant Sciences Seminar: Mutualism in Action (Toby Kiers and René Geurts)

11 Mar 2014

Jeroen Mesters 'From protein solution to single crystal X-ray diffraction: Chitin 31 Mar 2014 binding by LysM domains at atomic resolution'

Jane Parker 'Reprogramming cells for defence in plant innate immunity'

09 Apr 2014

Frank van Breusegem 'Plant Metacaspases'

09 Apr 2014

Yuanchao Wang "Dissecting the interaction between Phytophthora sojae and

16 Jul 2014 soybean: making sense out of signalling and effectors"

Joy Bergelson WEES seminar "Maintaining an ancient balanced polymorphism 26 Sep 2014 for resistance amidst diffuse intereactions"

Michael Freitag "Chromatin structure controls centromeres and secondary

21 Oct 2014 metabolism in filamentous fungi"

Ortrun Mittelsten Scheid "Genetics and epigenetics: a complex relationship"

19 Nov 2014

Kevin Foster WEES seminar "The evolution of cooperation and competition in

22 Jan 2015 microbes"

Yves van de Peer 'The evolutionary significance of gene and genome

03 Feb 2015 duplications'

Monica Höfte 'Towards understanding rice brown spot, a disease induced by

06 Feb 2015 physiological stress'

Chih-Hang Wu 'Helper NLR proteins of the NRC family in solanaceous plants'

05 Mar 2015

Gero Steinberg 'Long-distance endosome trafficking drives fungal effector production during plant infection'

05 Jun 2015 


\begin{tabular}{|c|c|c|}
\hline & Gert Kema application as Special Professor in Tropical Phytopathology & 17 Nov 2015 \\
\hline & \multicolumn{2}{|l|}{ Symposia: } \\
\hline & Plantum 'Intraspecific Pathogen Variation - Implications and Opportunities' & 22 Jan 2013 \\
\hline & Minisymposium: How to write a world-class paper & 17 Oct 2013 \\
\hline & KNPV fall meeting 'The process to progress' & 27 Nov 2013 \\
\hline & $\begin{array}{l}\text { Minisymposium: Responses of plants to pathogenic micro-organisms and their } \\
\text { secreted proteins }\end{array}$ & 02 Dec 2013 \\
\hline & $\begin{array}{l}\text { Farewell Address Prof. dr. ir. Pierre de Wit - Fungal plant pathogens and the } \\
\text { plant immune system }\end{array}$ & 05 Jun 2014 \\
\hline & All-inclusive Breeding: Integrating high-throughput science & 16 Oct 2014 \\
\hline & Minisymposium: Phytopathology & 24 Nov 2014 \\
\hline & Meeting BU Biointeractions \& Plant Health - Phytopathology WU & 10 Feb 2015 \\
\hline \multirow[t]{3}{*}{ 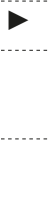 } & Seminar plus & \\
\hline & $\begin{array}{l}\text { Michael Freitag "Chromatin structure controls centromeres and secondary } \\
\text { metabolism in filamentous fungi" }\end{array}$ & 21 Oct 2014 \\
\hline & $\begin{array}{l}\text { Gero Steinberg 'Long-distance endosome trafficking drives fungal effector } \\
\text { production during plant infection' }\end{array}$ & 05 Jun 2015 \\
\hline$\rightarrow$ & \multicolumn{2}{|l|}{ International symposia and congresses } \\
\hline & 2nd joined Wageningen - Marburg meeting on plant-fungal interactions & 30-31 Jan 2012 \\
\hline & The 27th Fungal Genetics Conference 2013, Asilomar, California, USA & 12-17 Mar 2013 \\
\hline & The 28th Fungal Genetics Conference 2015, Asilomar, California, USA & 17-22 Mar 2015 \\
\hline \multirow[t]{10}{*}{ 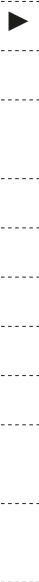 } & Presentations & \\
\hline & Poster: 27th Fungal Genetics Conference 2013, Asilomar, California, USA & $15 \operatorname{Mar} 2013$ \\
\hline & $\begin{array}{l}\text { Poster: Symposium "All-inclusive Breeding: Integrating high-throughput } \\
\text { science", Wageningen, NL }\end{array}$ & 16 Oct 2014 \\
\hline & Poster: 28th Fungal Genetics Conference 2015, Asilomar, California, USA & 17-22 Mar 2015 \\
\hline & Plant Science Slam: Annual meeting 'Experimental Plant Sciences', Lunteren, NL & 22 Apr 2013 \\
\hline & Talk: 7th International Utrecht PhD Summer School, Utrecht, NL & 27 Aug 2013 \\
\hline & Talk: KNPV fall meeting 'The process to progress', Wageningen, NL & 26 Nov 2013 \\
\hline & Talk: 6th European Plant Science Retreat, UvA, Amsterdam, NL & $03 \mathrm{Jul} 2014$ \\
\hline & $\begin{array}{l}\text { Flash: Symposium 'All-inclusive Breeding: Integrating high-throughput } \\
\text { science', Wageningen, NL }\end{array}$ & 16 Oct 2014 \\
\hline & $\begin{array}{l}\text { Talk: EPS Theme 2: Interactions between Plants and Biotic Agents \& Willie } \\
\text { Commelin Scholten Day Utrecht, NL }\end{array}$ & 20 Feb 2015 \\
\hline$\triangleright$ & \multicolumn{2}{|l|}{ IAB interview } \\
\hline \multirow[t]{2}{*}{ 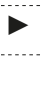 } & Excursions & \\
\hline & Company visit: Rijk Zwaan de Lier & 27 Sep 2013 \\
\hline
\end{tabular}

Subtotal Scientific Exposure 24.9 credits* 


\begin{tabular}{l}
$\begin{array}{l}\text { 3) In-Depth Studies } \\
\text { EPS courses or other PhD courses }\end{array}$ \\
7th International Utrecht PhD Summer School on Environmental Signaling, \\
Utrecht, NL \\
\hline Journal club \\
\hline Participitated in the literature discussion of the Verticillium group \\
\hline Individual research training
\end{tabular}

\begin{tabular}{|c|c|c|}
\hline & Subtotal In-Depth Studies & 3.9 credits* \\
\hline \multicolumn{2}{|c|}{$\begin{array}{l}\text { 4) Personal development } \\
\text { Skill training courses }\end{array}$} & date \\
\hline & Course: PhD competence assessment & 20 Mar 2012 \\
\hline & Course: Scientific publishing & 19 Jun 2012 \\
\hline & Course: Project- and Time Management & 30 Oct-11 Dec 2012 \\
\hline & Course: Interpersonal Communication for PhD Students & $12-13$ Dec -2012 \\
\hline & WGS PhD Workshop Carousel 2015 & 17 Apr 2015 \\
\hline & Course: Last Stretch of the PhD Programme & 25 Sep 2015 \\
\hline 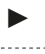 & \multicolumn{2}{|l|}{ Organisation of PhD students day, course or conference } \\
\hline & EPS Expectations day 2013 & 01 Feb 2013 \\
\hline & Company visit: Rijk Zwaan de Lier & 27 Sep 2013 \\
\hline & EPS Expectations day 2014 & $28 \operatorname{Mar} 2014$ \\
\hline & Company visit: Genetwister and In2Care Wageningen & 19 Sep 2014 \\
\hline & EPS PhD student day (GET2GETHER) & 29-30 Jan 2015 \\
\hline 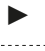 & \multicolumn{2}{|l|}{ Membership of Board, Committee or PhD council } \\
\hline & EPS PhD council member & $2012-2015$ \\
\hline & Subtotal Personal Development & 7.7 credits* $^{*}$ \\
\hline \multicolumn{2}{|r|}{ TOTAL NUMBER OF CREDIT POINTS* } & 45.0 \\
\hline
\end{tabular}

Herewith the Graduate School declares that the PhD candidate has complied with the educational requirements set by the Educational Committee of EPS which comprises of a minimum total of 30 ECTS credits

${ }^{*}$ A credit represents a normative study load of 28 hours of study. 
This work was supported by a VICl grant from the Research Council for Earth and Life sciences (ALW) of the Netherlands Organization for Scientific Research (NWO). The work was carried out in the Laboratory of Phytopathology, Wageningen University, the Netherlands.

Cover design: Iliana Boshoven-Gkini I AgileColor.com Thesis layout: Iliana Boshoven-Gkini I AgileColor.com Print: GVO drukkers \& vormgevers I gro.nl 
